\title{
A Study of Central Exclusive Production
}

A thesis submitted to the University of Manchester for the degree of Doctor of Philosophy in the Faculty of Engineering and Physical Sciences

2006

James Monk

School of Physics and Astronomy 


\section{Contents}

1 Introduction 12

2 Theoretical Motivation $\quad 14$

2.1 The Standard Model of Particle Physics . . . . . . . . . . . . . . 14

2.2 Supersymmetry . . . . . . . . . . . . . . . . . 21

2.3 QCD at Hadron Colliders . . . . . . . . . . . . . . . 24

2.4 Central Exclusive Production . . . . . . . . . . . . . . . 27

3 Exotic uses for Central Exclusive Production 34

3.1 A CP Violating Supersymmetric Higgs Boson . . . . . . . . . . . 34

3.1.1 Cross Section Calculation for CPX Higgs Boson Production 40

3.1.2 Results for Central Production of a CP Violating Higgs Boson 43

3.2 Warped Extra Dimensions . . . . . . . . . . . . . . 43

3.2.1 Cross Section Calculation for Central Exclusive Radion Production . . . . . . . . . . . . . 47

3.2.2 Results for Central Exclusive Radion Production . . . . . 47

3.2 .3 Discussion . . . . . . . . . . . . . . . . 48

4 The ExHuME Generator

4.1 Motivation . . . . . . . . . . . . . . . . . . 51

4.2 Monte Carlo Integration . . . . . . . . . . . . . . . 51

4.3 The Implementation of the Differential Luminosity . . . . . . . . 54

4.4 Soft Survival Factor . . . . . . . . . . . . . . . . . . 60

4.4.1 Sub Processes . . . . . . . . . . . . . . . 60 
4.5 Event Generation . . . . . . . . . . . . . . . . . 61

4.5.1 Parton Shower and Hadronisation . . . . . . . . . . 66

4.6 Results from ExHuME . . . . . . . . . . . . . . . . . . . 67

4.6.1 Higgs Boson Production . . . . . . . . . . . . 67

4.6.2 Direct b Jet Production at the LHC . . . . . . . . . . 71

4.6.3 Di-Jet Production at the Tevatron . . . . . . . . . . 73

5 The DØ Experiment $\quad 81$

5.1 The Tevatron . . . . . . . . . . . . . . . . . . . . . . . . 81

5.2 The DØ Detector . . . . . . . . . . . . . . . . . . . 82

5.2.1 The Central Tracker . . . . . . . . . . . . . . . . . . 83

5.2 .2 The DØ Calorimeter . . . . . . . . . . . . 85

5.2 .3 The Luminosity Monitor . . . . . . . . . . . . . . 87

5.2 .4 The Muon System . . . . . . . . . . . . . . . . . 88

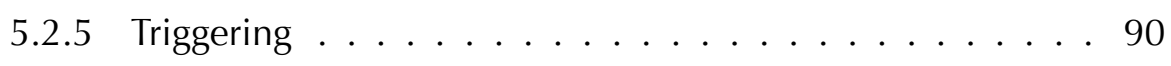

5.2.6 The Forward Proton Detector System . . . . . . . . . . 92

6 Proton Hit Patterns in the Forward Proton Detectors 97

6.1 Reconstruction of the Proton Hit Positions . . . . . . . . . . . . . . 98

6.2 Least Squares Fitting of an Ellipse . . . . . . . . . . . . . . 102

7 Search for Exclusive Di-Jets at DØ 110

7.1 Candidate Event Selection . . . . . . . . . . . . . . 111

7.1.1 Inclusive Background Simulated with Pomwig . . . . . . 111

7.1 .2 Triggering . . . . . . . . . . . . 112

7.1.3 Calorimeter Calibration and Noise . . . . . . . . . . 113

7.1 .4 Jet Finder . . . . . . . . . . . . . . . . 115

7.1.5 Gap Cut and $x$ Correction $\ldots \ldots \ldots \ldots$

7.1 .6 Kinematic Cuts . . . . . . . . . . . . . . . . 119

7.2 Monte Carlo Comparison . . . . . . . . . . . . . . . . . . . . . 120

7.3 Threshold for Particle Detection . . . . . . . . . . . . . 120

7.4 Summary of Analysis cuts . . . . . . . . . . . . . . . . . 121

7.5 Results . . . . . . . . . . . . . . . . . . . . 122 
7.6 Discussion . . . . . . . . . . . . . . . . . . 132

8 Concluding Remarks 134

A The ExHuME Monte Carlo 136

A.1 Design . . . . . . . . . . . . . . . . . . 136

A.2 The CrossSection Class . . . . . . . . . . . . . . . . . 136

A.3 The Event Class . . . . . . . . . . . . . . . . . . . . . . . . . 140

A.4 Using ExHuME . . . . . . . . . . . . . . . . . . . . . 142

A.5 The ExHuME Control Parameters . . . . . . . . . . . . . . . 145 


\section{List of Figures}

1 Thanks! . . . . . . . . . . . . . . . . . . . 11

2.1 Loops in the gluon propagator . . . . . . . . . . . . 20

2.2 A proton collision . . . . . . . . . . . . . . 25

2.3 Central exclusive production of a system $\Gamma \ldots \ldots \ldots$

2.4 Cutting the intermediate quark lines in the diagram for central exclusive production. . . . . . . . . . . . . . . . 29

3.1 MSSM Higgs Mixing $\ldots \ldots \ldots$

3.2 Higgstrahlung at the LEP collider. . . . . . . . . . . . . 37

3.3 Regions of MSSM parameter space excluded by LEP . . . . . . . . 39

3.4 Higgs boson production via gluon fusion. . . . . . . . . . . . 40

3.5 Cross section for central exclusive production of the lightest MSSM Higgs Boson . . . . . . . . . . . . . . . . . . . . 44

3.6 The ratio of the form factor $S^{1}$ in the CPX scenario to that in the Standard Model . . . . . . . . . . . . . . . . . . . . . . . . . 44

3.7 Cross section for central exclusive production for the Higgs Boson and Radion in the Randall Sundrum Model . . . . . . . . . . . . 48

3.8 Central exclusive cross sections for $\phi \rightarrow b \bar{b}$ and $h \rightarrow b \bar{b} \ldots \ldots 49$

4.1 Calculating $\pi$ from random points within a square $\ldots \ldots . . .52$

4.2 Using Monte Carlo techniques to integrate a differential cross section 53

4.3 The Sudakov suppression as implemented in ExHuME . . . . . . 56

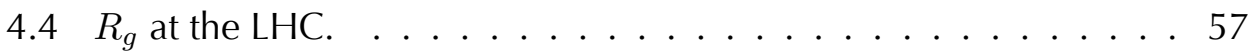

4.5 The unintegrated gluon distributions as implemented in ExHuME . 58 
4.6 The mass and rapdidity dependence of the differential luminosity taken from ExHuME . . . . . . . . . . . . . . 58

4.7 The mass and rapidity dependence of the differential luminosity taken from ExHuME . . . . . . . . . . . . . . . . . . 59

4.8 Producing a Breit-Wigner distribution for $\hat{s}$ from a set of random numbers distributed uniformly in $\{0,1\} \ldots \ldots$. . . . . . 62

4.9 Random number distribution from ExHuME . . . . . . . . . . . . 64

4.10 A resonant differential cross section and an approximate fit to it. . 64

4.11 Convergence of ExHuME over 10,000 events . . . . . . . . . . . . 66

4.12 Standard model Higgs boson production cross section taken from

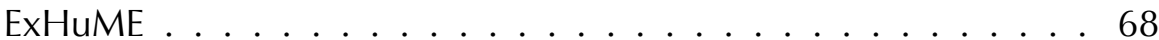

4.13 Mass of the centrally produced $120 \mathrm{GeV}$ Higgs boson . . . . . . . 69

4.14 Rapdity distributions for Higgs boson production in ExHuME . . . 70

4.15 The $x$ distributions for a $120 \mathrm{GeV}$ Higgs boson. . . . . . . . . . . . 71

4.16 The $t$ distribution for a $120 \mathrm{GeV}$ Higgs boson. . . . . . . . . . . . 72

4.17 Jet $E_{T}$ for Higgs boson production and its background . . . . . . 73

4.18 The statistical significance in $\sigma$ per $\mathrm{fb}^{-1 / 2}$ of integrated luminosity ${ }^{1 / 2} 74$

4.19 The total central mass from ExHuME $q \bar{q}$ and $g g$ events with an allowed mass range of 14 to $80 \mathrm{GeV} \ldots \ldots$. . . . . . . . 75

4.20 The $E_{T}$ distributions of the largest jet from ExHuME $\ldots \ldots$. . . 76

4.21 The largest (left) and smallest (right) $x$ in the event. . . . . . . . . 77

4.22 The rapidity distributions for $q \bar{q}$ and $g g$ production $\ldots \ldots . . .78$

4.23 The particle and Jet multiplicities f . . . . . . . . . . . . 79

4.24 The di-jet mass fraction from ExHuME . . . . . . . . . . 80

5.1 The Tevatron . . . . . . . . . . . . . . . . . . . . . . . 82

5.2 The DØ detector. . . . . . . . . . . . . . . . . . . 83

5.3 The silicon microstrip tracker . . . . . . . . . . . . . . 85

5.4 The calorimeter in cross section . . . . . . . . . . . . . . 87

5.5 Calorimeter energy resolution $\ldots \ldots$. . . . . . . . 88

5.6 The luminosity monitor . . . . . . . . . . . . . . . . . . . 88

5.7 The muon tracking system $\ldots \ldots \ldots$. . . . . . . . . 90 
5.8 The FPD system at $\mathrm{D} \varnothing \ldots \ldots \ldots$

5.9 Photograph of damage caused to a Tevatron beam collimator . . . 95

5.10 The $u, v$ and $x$ planes of fibres. . . . . . . . . . . . . 96

6.1 The intersection of three nearby fibres . . . . . . . . . . . . 99

6.2 Proton flux patterns in the Roman pots . . . . . . . . . . 101

6.3 Enhancement in the proton flux image by selecting events with single pot hits . . . . . . . . . . . . . . 102

6.4 Pot window divided into a $10 \times 10$ grid $\ldots \ldots$. . . . . . . 103

6.5 A set of points in the pot window all with the same proton flux. . . 104

6.6 Ellipes fitted to ten smeared points . . . . . . . . . . . . 105

6.7 Ellipses Fitted to Monte Carlo and data samples of pot fibre hits . . 107

7.1 A ladder of gluons. . . . . . . . . . . . . . . . . . 112

7.2 Production of a di-jet system through double Pomeron exchange . 113

7.3 The Level 3 jet trigger efficiency . . . . . . . . . . . . . . . 114

7.4 A "Ring of Fire" noise signal in the calorimeter. . . . . . . . . . . . 116

7.5 The fraction of events with $x_{c a l} / x_{t r u}<0.7$ versus the rapidity gap cut that is made. . . . . . . . . . . . . . . . . 118

7.6 A scatter plot of the ratio $x_{c a l} / x_{t r u}$ versus $x_{c a l} \ldots \ldots$. . . . 119

7.7 The angle $\phi_{j j}$ between the two leading jets in data and Pomwig . . 125

7.8 The observed $R_{j j}$ distribution and the $R_{j j}$ calculated in events generated by Pomwig with particle thresholds of $0.5,1,2$, and 4

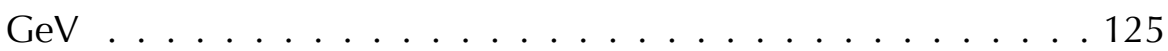

7.9 The observed tower energy spectrum compared to Pomwig . . . . 126

7.10 The number of jets in each event . . . . . . . . . . . 126

7.11 The $R=0.015$ jet multiplicity . . . . . . . . . . . . . 127

7.12 The di-jet mass fraction $R_{j j} \quad \ldots \ldots \ldots \ldots \ldots \ldots$

7.13 The di-jet mass fraction $R_{j j} \ldots \ldots \ldots \ldots \ldots \ldots$

7.14 The di-jet mass fraction $R_{j j} \ldots \ldots \ldots \ldots \ldots$

7.15 The rapidity distribution of the central system in data, Pomwig and

ExHuME. . . . . . . . . . . . . . . . . . 129 
7.16 The maximum and minimum $x_{c a l}$ distributions . . . . . . . 129

7.17 The number of events in which the leading jet had transverse energy above $E_{T}$ and $R_{j j}>0.85 \ldots \ldots \ldots \ldots \ldots$

7.18 The energy deposition in the calorimeter from an event with $R_{j j}=0.68$ (top) and $R_{j j}=1$ (bottom) $\ldots \ldots \ldots \ldots$. . . . . . . . 


\section{Abstract}

Central exclusive production of a system $X$ in a collision between two hadrons $h$ is defined as $h h \rightarrow h+X+h$ with no other activity apart from the decay products of $X$. This thesis presents predictions for the production cross section of a CP violating supersymmetric Higgs boson and the radion of the Randall-Sundrum model.

The ExHuME Monte Carlo generator was written to simulate central exclusive processes and is described and explored. A comparison to di-jet observations made by the $\mathrm{D} \varnothing$ detector at the Tevatron, Fermilab between January and June 2004 is made and the distributions found support the predictions of ExHuME. 


\section{Declaration}

No portion of the author's work described in this thesis has been submitted in support of an application for another degree or qualification in this, or any other, institute of learning.

\section{Copyright Statement}

(i) Copyright in text of this thesis rests with the Author. Copies (by any process) either in full, or of extracts, may be made only in accordance with instructions given by the Author and lodged in the John Rylands University Library of Manchester. Details may be obtained from the Librarian. This page must form part of any such copies made. Further copies (by any process) of copies made in accordance with such instructions may not be made without the permission (in writing) of the Author.

(ii) The ownership of any intellectual property rights which may be described in this thesis is vested in the University of Manchester, subject to any prior agreement to the contrary, and may not be made available for use by third parties without the written permission of the University, which will prescribe the terms and conditions of any such agreement.

(iii) Further information on the conditions under which disclosures and exploitation may take place is available from the Head of School of Physics and Astronomy. 


\section{Acknowledgements}

Thanks to all the people who made the past three years so much fun, and sometimes even productive. It is said that a picture is worth a thousand words (figure 1).
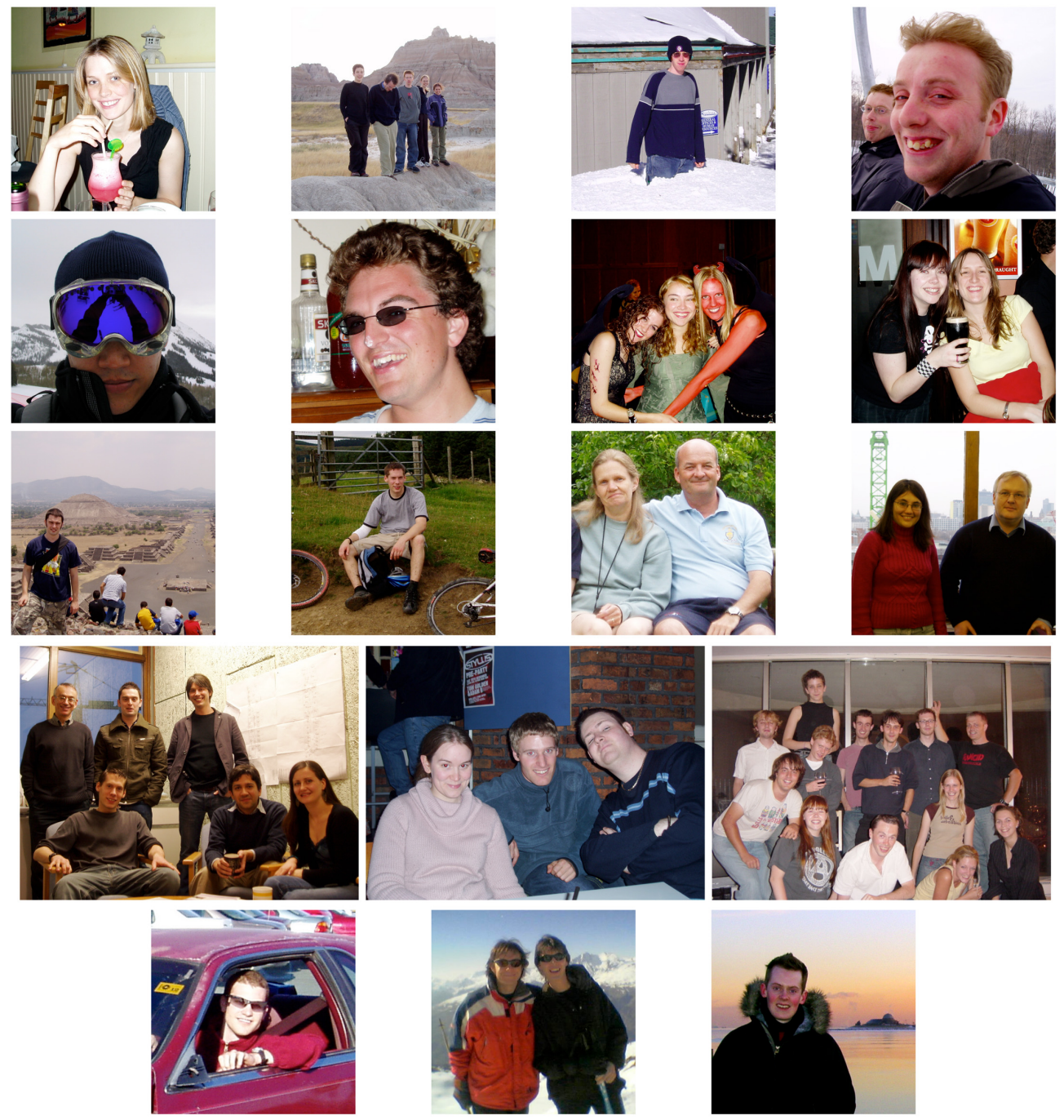

Figure 1: Thanks! 


\section{Chapter 1}

\section{Introduction}

High energy particle colliders probe the structure and interactions of matter at the smallest scale possible. Hadron colliders offer the highest centre of mass collision energy but, due to the composite structure of the proton and additional interactions between the partons within the proton, they do not provide as clean an environment as lepton colliders. Hadron collision events are overlayed with a background QCD interaction that results from the disrupted hadrons. In addition, knowledge of the structure of the hadron is required in order to interpret observations.

Central exclusive production [1-4], introduced in chapter 2, provides a clean measuring environment for hadronic collisions. In a central exclusive event the beam hadrons remain intact, rather than dissociating as normally happens in a hadron collision. The outcome of a central exclusive event is therefore the central system of interest (the mass of which shall be referred to as the central mass) and two outgoing hadrons. Due to the fact that the outgoing protons remain intact, the central system has its charge $(C)$ and parity $(\mathrm{P})$ quantum numbers constrained. In addition, the component of intrinsic angular momentum in the beam direction $\left(J_{z}\right)$ is also constrained. The advantages of central exclusive production; reduced QCD background; selection of $J_{z}=0$ states and precise measurement of the central mass via the missing mass method [5] may allow the study of states 
that are otherwise hard to observe. Two such states; a CP violating Minimally Supersymmetric Standard Model (MSSM) Higgs boson [6] and the radion of the Randall-Sundrum model [7] are explored in the context of central exclusive production in chapter 3 .

The missing mass method for determining the central mass requires that both outgoing intact protons are tagged in proton detectors downstream from the central detector. Such a system of proton taggers has been installed at the D $\varnothing$ detector (chapter 5) of Fermilab and is described in chapter 6. One of the problems for the moveable proton taggers is knowing the precise position of the proton beam in relation to the tagger, and chapter 6 also discusses an analysis of the beam position using the data taken by the tagger itself.

Whilst good evidence for the existence of central exclusive production has now been collected $[8,9]$, the process has never conclusively been observed. One channel with a sufficiently large cross section to be observable at the Tevatron is the exclusive production of a pair of di-jets. In order to aid the comparison of the so-called Durham calculation of the exclusive process [2] to data the ExHuME Monte Carlo simulation [10] was written, which is discussed in full in chapter 4 .

ExHuME allows predictions from the Durham calculation to be compared to data currently available from both the D $\varnothing$ and CDF experiments and chapter 7 presents the first comparison to data taken at $\mathrm{D} \varnothing$. It is found that the distributions predicted by ExHuME agree with the data. 


\section{Chapter 2}

\section{Theoretical Motivation}

\subsection{The Standard Model of Particle Physics}

The Standard Model (SM) of particle physics, which describes the structure of matter at the smallest scales yet probed, is built upon a foundation of symmetry. Lorentz symmetry, under which the world line element $d s$ with Minkowski metric $\eta_{\mu \nu}$ and coordinates $x^{\mu}[11]$

$$
d s^{2}=\eta_{\mu \nu} d x^{\mu} d x^{\nu}
$$

is invariant, provides the group with representations that are (among others) the spin $1 / 2$ fermion fields and the spin 1 vector boson fields. Lorentz scalar fields that are invariant under Lorentz transformations are also allowed, but no fundamental Lorentz scalar has ever been observed.

The interactions between the fields are described by requiring that the Lagrangian density of the Standard Model be invariant under the three local distinct gauge transformations [12]

$$
U(1)_{Y} \otimes S U(2)_{L} \otimes S U(3)_{C}
$$

where the subsript $L$ indicates that the $S U(2)$ transformation acts on a left-handed $S U(2)$ doublet. A fermion field that carries hyper-charge $Y$, colour charge $C$ or carries weak isospin charge transforms as follows: 


$$
\begin{array}{cccc}
\psi & \mapsto \quad e^{i Y \phi\left(x^{\mu}\right)} \psi & U(1)_{Y}, \\
\psi & \mapsto & e^{i \frac{\tau_{\mathbf{N}}}{2} \cdot \alpha_{N}\left(x^{\mu}\right)} \psi & S U(N)
\end{array}
$$

where $\tau_{N}$ are the $2 \times 2$ or $3 \times 3$ unitary generator matrices of $S U(2)$ or $S U(3)$ and $\phi\left(x^{\mu}\right)$ and $\alpha\left(x^{\mu}\right)$ are fields specifying the gauge transformations. Under the same transformation the Lagrangian density for a massless fermion becomes:

$$
\begin{array}{llll}
\Delta \mathcal{L}_{f}=i \bar{\psi} \not \partial \psi & \mapsto \quad i \bar{\psi}\left(\not \partial+i Y \not \partial \phi\left(x^{\mu}\right)\right) \psi & U(1)_{Y}, \\
\Delta \mathcal{L}_{f}=i \bar{\psi} \not \partial \psi & \mapsto i \bar{\psi}\left(\not \partial+i \frac{\tau}{2} \cdot \not \partial \alpha\left(x^{\mu}\right)\right) \psi & S U(N) .
\end{array}
$$

To cancel the derivative of $\phi\left(x^{\mu}\right)$ or $\alpha\left(x^{\mu}\right)$ the covariant derivative is defined that includes a new set of fields; the force carrying gauge bosons. For example, the derivative $\partial \phi\left(x^{\mu}\right)$ is cancelled by the field $B^{\mu}\left(x^{\nu}\right)$, which transforms under $U(1)$ as follows:

$$
B^{\mu} \mapsto B^{\mu}-\partial^{\mu} \phi
$$

and the covariant derivative, $D^{\mu}$, that renders the Lagrangian density invariant under $U(1)$ transformations is

$$
D^{\mu}=\partial^{\mu}+i Y B^{\mu} .
$$

When the covariant derivative is used in place of $\not \partial$ in equation 2.4 the result is gauge invariant, that is independent of $\phi\left(x^{\mu}\right)$ and $\alpha_{N}\left(x^{\mu}\right)$

$$
\Delta \mathcal{L}_{f}=i \bar{\psi}\left(\not \partial+i g_{s} A T_{a}^{i j}+i g \tau \cdot \not{W}+i \frac{g^{\prime}}{2} Y \not B\right) \psi
$$

which now has the gauge bosons of the gluon field, $A$, two charged $W^{ \pm}$fields, a neutral $W^{3}$ field and a neutral $B$ field. Fields only couple to a gauge boson if they carry the requisite charge so that the generator of the gauge boson $\left(T_{a}^{i j}, \tau\right.$ or $Y)$ returns a non-zero result when it acts on the field.

The fermions of the Standard Model are categorised according to the charges that they carry. Chirality, $\chi$, is the projection of either the upper or lower component of the fermion representation of the Lorentz group: 


$$
\chi_{ \pm} \psi=\frac{1}{2}\left(1 \pm \gamma_{5}\right) \psi
$$

A particle with $\left\{\chi_{+}, \chi_{-}\right\}=\{0,1\}$ or $\left\{\chi_{+}, \chi_{-}\right\}=\{1,0\}$ is said to be left-handed or right-handed respectively. The eigenstates of chirality for a massless fermion coincide with the eigenstates of helicity, $h$, the projection of a particle's intrinsic angular momentum onto its momentum, $\mathbf{p}$ :

$$
h \psi=\frac{\boldsymbol{\Sigma} \cdot \mathbf{p}}{|\boldsymbol{\Sigma} \cdot \mathbf{p}|} \psi
$$

where $\boldsymbol{\Sigma}$ is the angular momentum operator. In the Standard Model only lefthanded particles carry weak isospin, so the three generators of $S U(2), \tau$, return zero when they operate on a right-handed particle $\psi_{R}$.

$$
\tau \psi_{R}=0
$$

The opposite is true of anti-particles; right-handed anti-particles carry weak isospin, whereas left handed anti-particles do not.

The only fermions that possess colour charge, and hence couple to the gluon field, are the quarks, of which there are six in total. The left-handed quarks are arranged into three $S U(2)$ doublets, while the right-handed quarks are six $S U(2)$ singlet states. Fermions that do not carry colour charge are called leptons, of which there are six. As with the quarks, the left-handed leptons are arranged into three $S U$ (2) doublets: the electron and the electron neutrino $\left(\nu_{e}\right)$; the muon and the muon neutrino $\left(\nu_{\mu}\right)$ and the tau and the tau neutrino $\left(\nu_{\tau}\right)$. The quantum numbers of the Standard Model fermions are shown in table 2.1.

Equation 2.7 does not include any mass terms for either the gauge bosons or the fermions, which is at odds with the experimental observation that the two charged $W^{ \pm}$bosons have a mass of $80.43 \mathrm{GeV}$ [13], there is a neutral gauge boson - the $Z^{0}$ - with a mass of $91.2 \mathrm{GeV}$ and all of the quarks and the electron, muon and tau have a mass. Neutrinos were for a long time thought to be massless, but observation of oscillations between different neutrino species resulting from neutrino mixing [14] implies that at least two of the three neutrino species also 


\begin{tabular}{|c|c|c|c|c|c|c|}
\hline & & & Particle & & Hypercharge & Isospin \\
\hline & & Up & Charm & Top & $1 / 3$ & $1 / 2$ \\
\hline & Quarks & & & & & \\
\hline Left & & Down & Strange & Bottom & $1 / 3$ & $-1 / 2$ \\
\hline Handed & & $\nu_{e}$ & $\nu_{\mu}$ & $\nu_{\tau}$ & -1 & $1 / 2$ \\
\hline & & $\mathrm{e}_{L}$ & $\mu_{L}$ & $\tau_{L}$ & -1 & $-1 / 2$ \\
\hline & & Up & Charm & Top & $4 / 3$ & 0 \\
\hline Right & & Down & Strange & Bottom & $-2 / 3$ & 0 \\
\hline & Leptons & & & & & \\
\hline & & $\mathrm{e}_{R}$ & $\mu_{R}$ & $\tau_{R}$ & -2 & 0 \\
\hline
\end{tabular}

Table 2.1: The Standard Model fermions and their quantum numbers.

have mass.

Adding mass terms, such as $\Delta \mathcal{L}_{m_{W}}=m_{W}^{2} \overline{\mathbf{W}} \cdot \mathbf{W}$ to equation 2.7 breaks the gauge invariance unless the masses are added in a very specific way. A Lorentz scalar $S U(2)$ doublet field named the Higgs field, $\Phi$, with hyper-charge $Y=1$ is introduced that couples to the $\mathbf{W}$ field. The Lagrangian density, $\Delta \mathcal{L}_{H}$ for the Higgs field is:

$$
\begin{array}{r}
\Delta \mathcal{L}_{H}=\frac{1}{2}\left(\partial^{\mu}-i g \tau \cdot \mathbf{W}^{\mu}-i \frac{g^{\prime}}{2} Y B^{\mu}\right) \bar{\Phi}\left(\partial_{\mu}+i g \tau \cdot \mathbf{W}_{\mu}+i \frac{g^{\prime}}{2} Y B_{\mu}\right) \Phi \\
-\mu^{2} \bar{\Phi} \Phi-\lambda(\bar{\Phi} \Phi)^{2}
\end{array}
$$

If $\mu^{2}<0 \mathrm{GeV}^{2}$ and $\lambda>0$ then the potential energy for the Higgs field has a minimum when

$$
|\Phi|^{2}=-\frac{\mu^{2}}{2 \lambda}=\frac{v^{2}}{2}
$$

where $v$ is the Vacuum Expectation Value (VEV) for the Higgs field and the vacuum in its lowest energy state is filled with the Higgs field with amplitude $v / \sqrt{2}$. The term coupling the $\mathbf{W}$ to the $\Phi$ in equation 2.11 , when expanded about the vacuum, results in a mass for the $\mathbf{W}$ : 


$$
\Delta \mathcal{L}_{m_{W}}=\frac{1}{4} g^{2} v^{2} \tau \cdot \mathbf{W}^{\mu} \tau \cdot \mathbf{W}_{\mu}
$$

giving the mass of the $W, m_{W}$, as $m_{W}^{2}=g v^{2} / 4$. The quantum of the Higgs field, the Higgs boson, has a mass of $\mu / \sqrt{2}$, but has never been observed. An upper limit to the Higgs boson mass of $114 \mathrm{GeV}$ was obtained by experiments performed at the LEP collider [15]. The Higgs VEV, $v$, has been inferred from measurements of the $W^{ \pm}$mass and electroweak coupling constant, $g$, to be 246 GeV.

Masses for fermions are generated by a so-called "Yukawa" coupling between the left and right-handed fermion field and the Higgs field. The Yukawa term coupling a fermion to the Higgs field is:

$$
\Delta \mathcal{L}_{m_{f}}=g_{f} \bar{\psi}_{R} \bar{\Phi} \psi_{L}+\text { h.c. }
$$

where $g_{f}$ is the Yukawa coupling. The mass of the fermion is given by $m_{f}=g_{f} v$. In the case that the neutrinos are massless there is no right handed neutrino, so there is only one term like equation 2.14 for each lepton doublet. This is not the case for the quark sector, where a mass must be generated for both the up and down type quarks. If a gauge is chosen so that the ground state of the Higgs field is $\Phi_{0}$ :

$$
\Phi_{0}=\left(\begin{array}{c}
0 \\
v / \sqrt{2}
\end{array}\right)
$$

then $\Phi_{0}$ generates a mass for the down-type quarks. A transformation can be applied to the Higgs field:

$$
\begin{aligned}
\tilde{\Phi} & =\quad i \tau_{2} \Phi \\
\tilde{\Phi}_{0} & =\left(\begin{array}{c}
v / \sqrt{2} \\
0
\end{array}\right)
\end{aligned}
$$

so that $\tilde{\Phi}_{0}$ generates a mass for the up-type quarks and the Yukawa terms for the 
quarks are:

$$
\Delta \mathcal{L}_{m_{q}}=g_{u} \bar{u}_{R} \tilde{\Phi} Q_{L}^{\prime}+g_{d} \bar{d}_{R} \Phi Q_{L}^{\prime}+\text { h.c. }
$$

where $g_{u}$ and $g_{d}$ are the up and down quark Yukawa couplings, $u_{R}$ and $d_{R}$ are right-handed up and down $S U(2)$ singlets and $Q_{L}^{\prime}$ is a left-handed $S U(2)$ quark doublet. The weak eigenstate $Q_{L}^{\prime}$ is not an eigenstate of mass, with the result that the mass eigenstates of the quarks are a superposition of weak eigenstates. The $3 \times 3$ unitary matrix that transforms the weak eigenstate $Q_{L}^{\prime}$ to the mass eigenstate $Q_{L}$ is called the Cabbibo-Kobayashi-Maskawa (CKM) matrix and is determined by three angles and a single complex phase. The mixing means that the propagating mass eigenstates of certain mesons, the $B_{0}$ for example, contain a small component of the anti meson $\left(\bar{B}_{0}\right)$ weak eigenstate. The complex phase of the CKM matrix results in a phase difference between the weak particle and anti-particle content of the meson, which in turn means that the mass eigenstate is not an eigenstate of the $C P$ operator. The rate of decay to a $C P$ eigenstate, such as a pair of neutral pions, is then different for the $B_{0}$ and $\bar{B}_{0}$. This effect is called $C P$ violation and can only occur if there are at least three generations of quarks.

The weak $S U(2)$ and strong $S U(3)$ forces are non-Abelian, that is, there are self interaction terms for the appropriate gauge boson. The terms in the Lagrangian density that produce interactions between gluons are:

$$
\Delta \mathcal{L}_{g g}=-\frac{1}{4} F_{a}^{\mu \nu} F_{\mu \nu}^{a}
$$

where $F_{\mu \nu}^{a}=\partial_{[\mu} A_{\nu]}+g_{s} f^{a b c} A_{\mu b} A_{\nu c}$ and $f^{a b c}$ are the structure constants for $S U(3)$. The structure constants describe the gauge structure of the group through a commutation relation with the generators of the group $T^{a}$

$$
\left[T^{b}, T^{c}\right]=i f^{a b c} T_{a}
$$

An important result of the gluon self-interaction is anti-screening of colour charge. Diagrams such as those in figure 2.1 cause the colour charge observed 

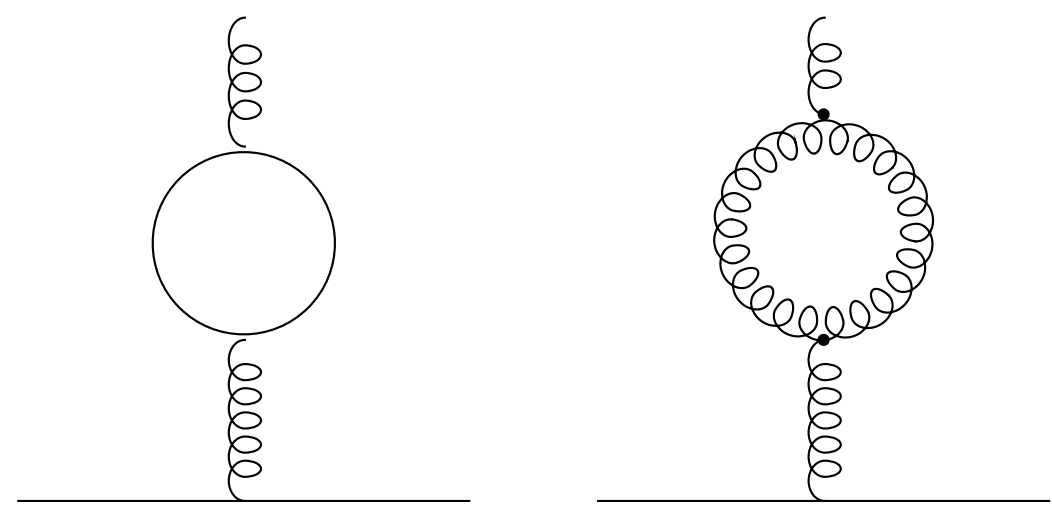

Figure 2.1: Loops in the gluon propagator produce a running coupling. The left hand diagram reduces the effective coupling at lower gluon energy, while the dominant right hand diagram enhances the effective coupling at higher gluon energy.

at long distances (in other words by long wavelength gluons) to be greater than the colour charge observed by shorter range interactions.

The diagram on the left of figure 2.1 contains a virtual $q \bar{q}$ dipole that reduces the field strength and screens the colour charge, while the diagram on the right with a virtual gluon pair produces anti-screening. The anti-screening effect is larger than the screening effect and, as a result, the strong coupling constant $\alpha_{s}=g_{s}^{2} / 4 \pi$ becomes larger as the energy scale at which an interaction occurs decreases. The running of $\alpha_{s}$ with scale $Q_{t}$ is approximately [16]

$$
\alpha_{s}\left(Q_{t}\right) \simeq \frac{2 \pi}{\left(11-\frac{2}{3} N_{f}\right) \ln \left(Q_{t} / \Lambda\right)}
$$

where $N_{f}$ is the number of coloured fermions with masses below $Q_{t}$ and $\Lambda_{Q C D}$ is a scale of $\mathcal{O}(100 \mathrm{MeV})$.

The coupling $\alpha_{s}$ diverges at $Q_{t}=\Lambda_{Q C D}$, therefore perturbative techniques cannot be used to carry out QCD calculations in the low energy regime. Another important feature of QCD that can be at least qualitatively explained by the running of $\alpha_{s}$ is confinement of the quarks. The gluon field between two quarks at a large separation becomes so strong that it is not possible to pull two quarks apart. A coloured object that is not bound to other coloured objects in a colour singlet state can never be produced. Such a colour singlet state can consist of an 
even number of quark fields in which for every field of colour charge $c_{i}$ there is a field of the anti-colour $\bar{c}_{i}$, for example, the colour state of a meson $\left(\left|\psi_{c}\right\rangle\right)$ can be written in terms of the colour states of two quark fields within the meson:

$$
\left|\psi_{c}\right\rangle=\sum_{i}\left|C_{i}\right\rangle\left|\bar{C}_{i}\right\rangle
$$

where the $\left|C_{i}\right\rangle$ are the colour states of the (anti) quark fields and the summation is over all three colour charges RGB. Alternatively, a colour singlet state can consist of an odd number of quarks (in the common case of a hadron this number is three) with all three (RGB) colours present and the overall system being antisymmetric to the exchange of any two colours.

For further details of the Standard Model please see [12, 16, 17].

\subsection{Supersymmetry}

There exists a further space-time symmetry that can be imposed upon the Lagrangian density over and above those symmetries of the Standard Model. This symmetry forces the Lagrangian to be invariant under transformations, $Q$, between fermionic and bosonic states $|f\rangle$ and $|b\rangle$ and is called supersymmetry (SUSY) [18]:

$$
\begin{aligned}
Q|f\rangle & =|b\rangle \\
Q \mathcal{L} & \mapsto \mathcal{L} .
\end{aligned}
$$

In order for this symmetry to be possible, each Standard Model particle must possess a super-partner with intrinsic angular momentum (spin) that differs by half a unit from the SM particle. The SM particles and their super-partners are arranged into so-called superfields. A scalar chiral supermultiplet consists of a complex spin zero scalar field paired with a spin 1/2 two component Weyl fermion. A four component fermionic field is composed of two Weyl spinors; one for the left-handed component and one for the right-handed. In the Minimally 
Supersymmetric Standard Model (MSSM) a scalar chiral superfield is therefore assigned to each of the left and right-handed SM fermionic fields, which therefore each possess a complex scalar superpartner field. For instance, the SM quarks are each partnered by two spin zero squarks in the MSSM.

A gauge superfield consists of a spin one bosonic field partnered by a spin 1/2 Weyl spinor. Each of the SM gauge bosons therefore possess a spin 1/2 super-partner in the MSSM. The SM gluon, for instance, is partnered by the spin $1 / 2$ gluino.

The Lagrangian of a supersymmetric theory can be specified by its superpotential. If $W$ is the superpotential then the Lagrangian density is:

$$
\Delta \mathcal{L}_{S U S Y}=-\frac{1}{2}\left(\frac{\partial^{2} W}{\partial \phi_{i} \partial \phi_{j}} \psi_{i} \psi_{j}+\left(\frac{\partial^{2} W}{\partial \phi_{i} \partial \phi_{j}}\right)^{*} \psi_{i}^{\dagger} \psi_{j}^{\dagger}\right)-\frac{\partial W}{\partial \phi_{i}}\left(\frac{\partial W}{\partial \phi_{i}}\right)^{*}
$$

where $\psi_{i}$ are two-component Weyl fermions and $\phi_{i}$ are the bosonic partners of $\psi_{i}$. The superpotential has no dependence on the fermionic fields, only the bosonic fields. For example, a superpotential that would generate a mass for a set of fermionic fields coupled to their bosonic partners by a Yukawa coupling is:

$$
W_{e x}=\frac{1}{2} M^{i j} \phi_{i} \phi_{j}+\frac{1}{6} y^{i j k} \phi_{i} \phi_{j} \phi_{k}
$$

where $M^{i j}$ is the mass matrix for the fermions, $y^{i j k}$ is the coupling between the $i^{\text {th }}$ and $j^{\text {th }}$ fermion flavours and the $k^{\text {th }}$ bosonic flavour and there is an implicit summation over the flavour indices $i, j$ and $k$.

The super-partners of the Standard Model fields have never been observed, which indicates that, if supersymmetry at the electroweak scale is a correct description of nature, the fermion/boson symmetry must be broken in some way. If it were not broken, the super-partners to the Standard Model would have precisely the same mass as the Standard Model particles and would have been observed. Breaking SUSY requires terms in the superpotential that provide additional mass for the unseen fields. Examples of the terms in the supersymmetric Lagrangian density that break the symmetry between fermions and bosons for a bosonic 
superpartner $\phi$ are $\Delta \mathcal{L}_{\text {break }}$ :

$$
\Delta \mathcal{L}_{\text {break }}=m_{s}^{2} \phi^{\dagger} \phi+\left(\frac{1}{2} B m \phi \phi+\frac{1}{6} A \phi \phi \phi+\text { h.c. }\right)
$$

where $m_{s}$ is a (soft) SUSY breaking mass and $B$ and $A$ are bilinear and trilinear couplings.

An attractive feature of supersymmetry is that it naturally solves the hierarchy problem of the Standard Model Higgs field. In the Standard Model, fermion loops in the Higgs propagator produce quadratically divergent corrections to the Higgs boson mass, $\Delta M_{h}$ :

$$
\Delta M_{h}^{2}=\frac{g_{f}^{2}}{16 \pi^{2}}\left(-2 \Lambda^{2}+6 m_{f}^{2} \ln \left(\Lambda / m_{f}\right)\right)
$$

where $m_{f}$ is the mass of the fermion in the loop, $g_{f}$ is its coupling to the Higgs field and $\Lambda$ is an ultraviolet cut-off to the loop integral; the scale at which some new and unknown interaction must be taken into account. A natural value for $\Lambda$ is the Planck scale, $M_{p} \sim 10^{19} \mathrm{GeV}$. In any case, it is reasonable to expect that interactions at the weak scale should not be affected by the very high energy behaviour of the theory above $\Lambda$. This is identical to the way that engineers of the nineteenth century were able to build successful steam engines based upon calculations in thermodynamics without having any knowledge of the underlying atomic structure or of the interactions of quantum electrodynamics. The correction in equation 2.26, however, is very sensitive to the scale of the unknown physics and this hierarchy between the electroweak scale, around which the Higgs boson mass lies, and the scale $\Lambda$ is known as the hierarchy problem.

Supersymmetry solves the hierarchy problem because it introduces a boson loop in the Higgs propagator for every Standard Model fermion loop. The boson loops exactly cancel the $\Lambda^{2}$ dependence of the Higgs boson mass, leaving the safe $\ln \left(\Lambda / m_{f}\right)$ dependence. 


\subsection{QCD at Hadron Colliders}

Hard collisions between protons at hadron colliders such as the Tevatron or LHC typically occur through the exchange of partons from within the beam particles. The low energy interactions between the partons inside the proton cannot be calculated perturbatively because $\alpha_{s}$ is large in that domain. The high energy interactions between the partons outside of the proton can, however, be treated perturbatively.

The procedure to calculate the cross section (equation 2.27) for a given process $X$ is therefore to calculate the so-called "hard scatter," the high energy interaction between the partons that constitute the proton and to fold it with a parton distribution function (PDF):

$$
d \sigma\left(h_{1} h_{2} \rightarrow X\right)=\sum_{i, j} \int d x_{1} d x_{2} f_{i}\left(x_{1}, \mu\right) f_{j}\left(x_{2}, \mu\right) d \hat{\sigma}(i j \rightarrow X)
$$

where $x_{1}$ and $x_{2}$ are the longitudinal momentum fractions of the parton species $i$ and $j$ from the hadrons $h_{1}$ and $h_{2}, f_{1}$ and $f_{2}$ are the PDFs for partons $i$ and $j$ and $\hat{\sigma}(i j \rightarrow X)$ is the cross section for the hard scatter.

The PDF specifies the parton content of the proton when probed at different scales. The PDF is dependent on both the scale at which the proton is probed, $\mu$, and the fraction of the proton's momentum that the parton carries, $x$. Physics below the scale $\mu$ is included in the PDF, whilst that above the scale of $\mu$ is part of the hard scatter and must be included in the perturbative calculation. The calculation has been factorised into non-perturbative and perturbative parts and for this reason $\mu$ is called the factorisation scale. If it were possible to calculate the hard scatter to all orders in perturbation theory then all dependence on $\mu$ would cancel from the calculation.

It is not possible to calculate the PDF perturbatively, but once it has been measured at a given $\mu$ it can be evolved to other scales using the DokshitzerGribov-Lipatov-Altarelli-Parisi (DGLAP) equations [19-21]: 


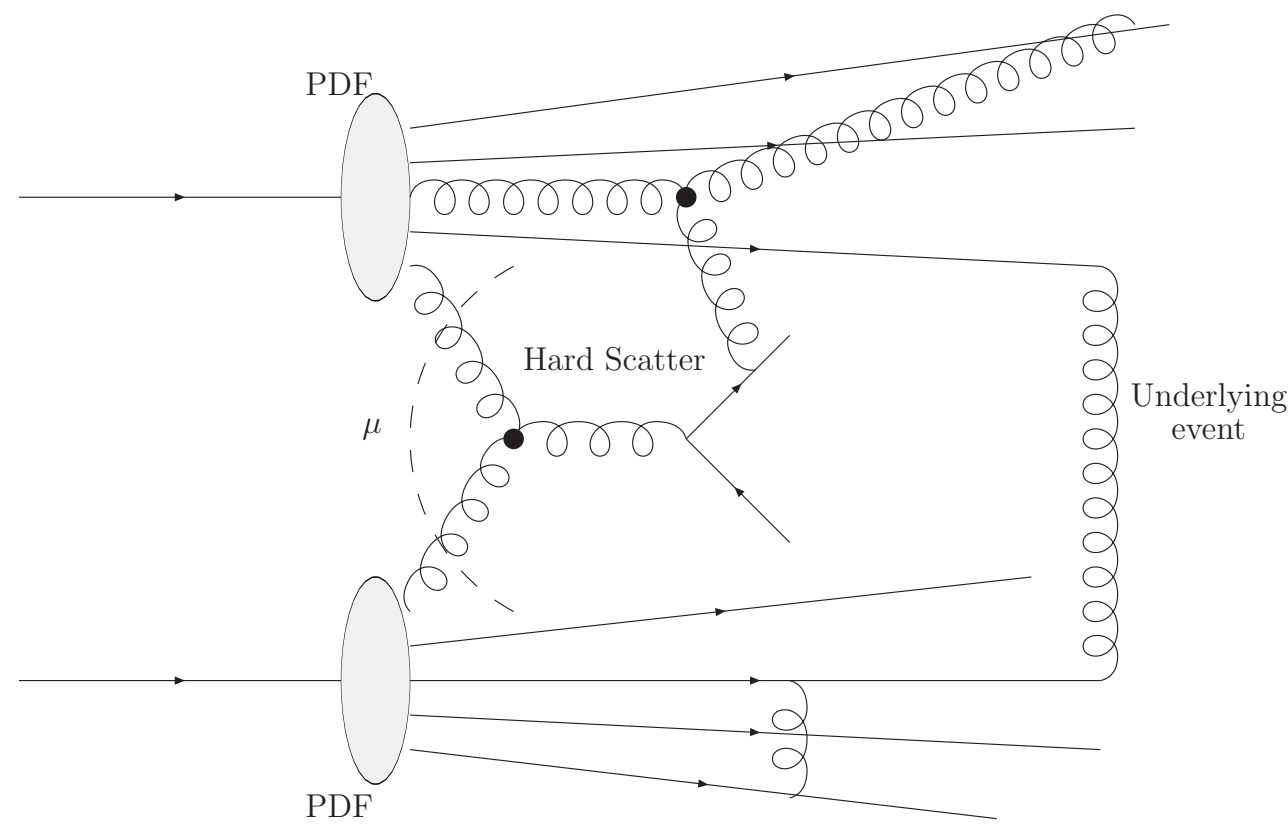

Figure 2.2: A proton collision factorised into a hard scatter above scale $\mu$, parton distribution functions and soft underlying event

$$
\begin{aligned}
& \frac{\partial q_{i}(x, \mu)}{\partial \ln (\mu)}=\frac{\alpha_{s}(\mu)}{2 \pi} \int_{x}^{1} \frac{d z}{z}\left(\sum_{j} q_{j}(z, \mu) P_{q_{i} q_{j}}(x / z)+g(z, \mu) P_{q_{i} g}(x / z)\right), \\
& \frac{\partial g(x, \mu)}{\partial \ln (\mu)}=\frac{\alpha_{s}(\mu)}{2 \pi} \int_{x}^{1} \frac{d z}{z}\left(\sum_{j} q_{j}(z, \mu) P_{g q_{j}}(x / z)+g(z, \mu) P_{g g}(x / z)\right)
\end{aligned}
$$

where $\alpha(\mu)$ is the (running) strong coupling constant at scale $\mu, q_{i}(x, \mu)$ and $g(x, \mu)$ are the distribution functions for the $i^{t h}$ species of quark and the gluon respectively and $P_{g g}, P_{q q}$ and $P_{q g}$ are the splitting functions giving the probability that a parton splits. At leading order the splitting functions are:

$$
P_{g g}(z)=6\left(\frac{1-z}{z}+\frac{z}{(1-z)_{+}}+z(1-z)\right),
$$




$$
\begin{aligned}
P_{q q}(z) & =\frac{4}{3} \frac{1+z^{2}}{(1-z)_{+}}, \\
P_{q g}(z) & =\frac{1}{2}\left(z^{2}+(1-z)^{2}\right)
\end{aligned}
$$

where the + subscript on the factor $1 /(1-z)_{+}$indicates that the so-called plus distribution should be used to remove the soft divergence at $z=1$. The plus distributions satisfy

$$
\int_{0}^{1} d z \frac{f(z)}{(1-z)_{+}}=\int_{0}^{1} d z \frac{f(z)-f(1)}{(1-z)} .
$$

At leading order it is only possible for a quark of species $j$ to split to another quark of the same species or a gluon, hence the summation over $j$ in equation 2.28 is not necessary at leading order. At higher order, however, the summation is necessary because the higher order splitting functions do permit splitting to a different species via an intermediate gluon line.

The parton distributions have been measured at the HERA electron-proton collider in deeply inelastic scattering [22] and those measurements (along with others) have been used to provide PDFs for both the Tevatron and the LHC (see for example $[23,24])$.

Removing a coloured parton completely disrupts the proton and leaves a proton remnant with an overall colour charge. This colour charge does not affect the hard scatter because the length scale on which the hard scatter occurs $(1 / \mu$ or shorter) is smaller than the scale on which the proton can resolve colour $(1 / \mu$ or longer). Each proton remnant system must, however, return to a colour singlet state and it does so through the exchange of colour with the other coloured objects in the collision. This process results in additional particle emission that can hinder the clean measurement of the hard scattering process. In addition, there may be soft radiation produced by multiple scatterings between the proton lines, which is known as the underlying event. 


\subsection{Central Exclusive Production}

The diagram for central exclusive production is shown in figure 2.3. There is no colour transferred from the two proton lines because a so-called "screening" gluon is exchanged to balance the colour transferred from each proton to the central hard system.

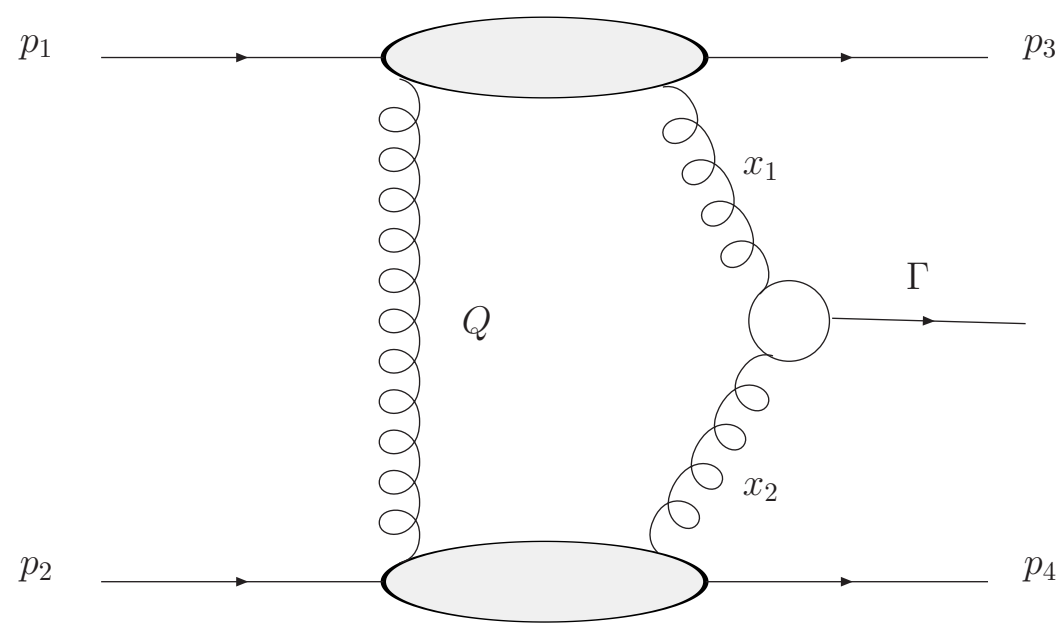

Figure 2.3: Central exclusive production of a system $\Gamma$

Throughout this thesis the following symbols will be used:

- $x_{1}=1-\frac{p_{3}^{0}}{p_{1}^{0}}$ is the fractional longitudinal momentum loss of proton 1 (upper line in figure 2.3)

- $x_{2}=1-\frac{p_{4}^{0}}{p_{2}^{0}}$ is the longitudinal momentum loss of proton 2 (lower line in figure 2.3)

- $y=\frac{1}{2} \log \left(x_{1} / x_{2}\right)$ is the rapidity of the central system

- $s=\left(p_{1}+p_{2}\right)^{2}$ is the centre of mass beam collision energy

- $t_{1}=\left(p_{3}-p_{1}\right)^{2}$ is the transverse momentum squared of outgoing proton 1

- $t_{2}=\left(p_{4}-p_{2}\right)^{2}$ is the transverse momentum squared of outgoing proton 2

- $\hat{s}=\left(p_{1}+p_{2}-p_{3}-p_{4}\right)^{2}$ is the central mass squared

- $\mu \sim \sqrt{\hat{s}} / 2$ is the factorisation or hard scale 
- $Q_{t}$ is the transverse momentum flowing around the loop in figure 2.3

The diffractive regime requires that $-t \ll \hat{s} \ll s$. The incoming and outgoing proton four-momenta can be written in terms of so-called Sudakov parameters

$$
\begin{aligned}
& p_{1}-p_{3}=\alpha_{1} p_{1}+\beta_{1} p_{2}+p_{\perp 1} \\
& p_{2}-p_{4}=\alpha_{2} p_{1}-\beta_{2} p_{2}+p_{\perp 2}
\end{aligned}
$$

where $p_{\perp 1}$ and $p_{\perp 2}$ are transverse to both incoming proton four-momenta, $p_{\perp 1}$. $p_{1}=p_{\perp 1} \cdot p_{2}=p_{\perp 2} \cdot p_{1}=p_{\perp 2} \cdot p_{2}=0$. In a proton collision at the TeV scale the proton mass can be neglected, so

$$
\begin{gathered}
p_{3}^{2}=-\left(1-\alpha_{1}\right) \beta_{1} s+p_{\perp 1}^{2} \simeq 0, \\
p_{4}^{2}=-\left(1-\beta_{2}\right) \alpha_{2} s+p_{\perp 2}^{2} \simeq 0, \\
-\frac{t_{1}}{s}=\alpha_{1} \beta_{1}+\frac{p_{\perp 1}^{2}}{s} \simeq \beta_{1} \ll 1, \\
-\frac{t_{2}}{s}=\alpha_{2} \beta_{2}+\frac{p_{\perp 2}^{2}}{s} \simeq \alpha_{2} \ll 1 . \\
\alpha_{1} \beta_{2}+\frac{p_{\perp 1} \cdot p_{\perp 2}}{s} \simeq \frac{\hat{s}}{s} \ll 1 .
\end{gathered}
$$

Therefore $1 \gg \alpha_{1} \gg \beta_{1}, 1 \gg \beta_{2} \gg \alpha_{2}, p_{\perp 1}^{2} \simeq t_{1}$ and $p_{\perp 2}^{2} \simeq t_{2}$. In the limit that the protons are massless then they are not able to change helicity and, given that the transverse momentum of the outgoing protons is very small, there can be no angular momentum transferred from the proton lines to the central system, which must therefore have zero spin projected on the z (beam) direction $\left(J_{z}=0\right)$, zero colour and zero charge.

The amplitude for figure 2.3 can be calculated with quarks replacing the protons by making use of the Cutkowsky rules [25]. These state that the imaginary part of the amplitude for a diagram with a loop, such as figure 2.3, can be calculated by cutting the diagram in two (figure 2.4), placing the cut lines onshell and integrating over their phase space. The Cutkowsky rules can be derived from a definition for the delta function, $\delta(z)$ : 


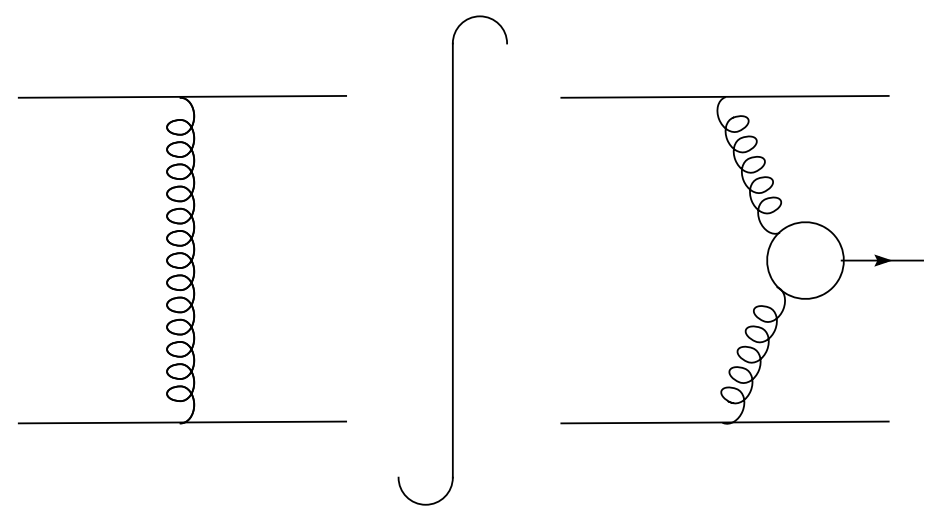

Figure 2.4: Cutting the intermediate quark lines in the diagram for central exclusive production.

$$
\delta(z)=\lim _{\epsilon \rightarrow 0} \frac{-1}{\pi} \Im m \frac{1}{z+i \epsilon} .
$$

The imaginary part of the amplitude for figure 2.4 is $\Im m(q \bar{q} \rightarrow q+\Gamma+\bar{q})$ $[26,27]:$

$$
\Im m(q \bar{q} \rightarrow q+\Gamma+\bar{q})=4 \int \frac{d^{2} Q_{t}}{Q_{t}^{4}} \frac{\left(N_{c}^{2}-1\right)}{\hat{s} N_{c}^{2}} \alpha_{s}^{2} \cdot \Gamma(\hat{s})
$$

where

$$
\Gamma(\hat{s})=\frac{1}{16} \sum_{\epsilon_{1}, \epsilon_{2}} \sum_{a, b} \delta^{a b} \epsilon_{1}^{\mu} \epsilon_{2}^{\nu} \mathcal{M}_{\mu \nu}^{a b}(g g \rightarrow \Gamma)
$$

is the amplitude for gluon fusion to $\Gamma$ averaged over the gluon polarisations, $\epsilon_{1}$ and $\epsilon_{2}$, and colours $a$ and $b$.

The parton-level cross section must be converted to a proton-level cross section by replacing the quark-gluon vertices with proton-gluon vertices. The un-integrated gluon distribution functions, $f\left(x, Q_{t}\right)$, give the gluon content with longitudinal momentum fraction $x$ at scale $Q_{t}$. The diagonal un-integrated gluon distribution function is given by $f_{d}$ :

$$
f_{d}\left(x, Q_{t}\right)=\frac{\partial g\left(x, Q_{t}\right)}{\partial \ln \left(Q_{t}^{2}\right)}
$$

where $g\left(x, Q_{t}\right)$ is the PDF evolved using the DGLAP equations (equation 2.28). 
Equation 2.37 is valid whenever the two gluons have the same longitudinal momentum fraction $x$. In the case of figure 2.3, the screening gluon has a much smaller $x$ than the other and this is corrected for with a factor $R_{g}$ [28] (see later section 4.3).

A crucial component of the conversion to a proton-level cross section is the Sudakov suppression factor, which gives the probability that the gluons attached to the central system $\Gamma$ do not emit additional radiation. The differential probability that a gluon at scale $\mu / 2$ emits radiation at a scale $k$ is given by $\partial P_{s}\left(k_{T, \mu / 2)} / \partial k_{T}^{2}\right.$ :

$$
\frac{\partial P_{s}}{\partial k_{T}^{2}}\left(k_{T, \mu} / 2\right)=\frac{\alpha_{s}\left(k_{T}\right)}{2 \pi k_{T}^{2}} \int_{0}^{\mu /\left(\mu+k_{T}\right)}\left(z P_{g g}(z)+P_{q g}(z)\right) d z
$$

where $P_{g g}(z)$ and $P_{q g}(z)$ are the splitting functions for a gluon to split to a pair of gluons or a quark and a gluon with momentum fraction $z$. The splitting function $P_{g g}$ makes the dominant contribution to equation 2.38 .

The Sudakov suppression factor, $T\left(Q_{t}, \mu\right)$ is determined by re-summing equation 2.38 integrated over all $k_{T}$ from $Q_{t}$ to $\mu / 2$ :

$$
\begin{aligned}
T\left(Q_{t}, \mu\right) & = \\
& \exp \left(-\int_{Q_{t}^{2}}^{(\mu / 2)^{2}} d k_{T}^{2} \frac{\alpha_{s}\left(k_{T}\right)}{2 \pi k_{T}^{2}} \int_{0}^{\mu /\left(\mu+k_{T}\right)}\left(z P_{g g}(z)+P_{q g}(z)\right) d z\right)
\end{aligned}
$$

The screening gluon cannot be resolved from the other two gluons by radiation below $Q_{t}$, which is therefore forbidden and justifies the lower limit to the integral in Eq. 2.39. The Sudakov suppression factor therefore only has to forbid radiation above $Q_{t}$. The Sudakov suppression is vital because, without it, equation 2.35 diverges in the infra-red as $Q_{t} \rightarrow 0$. The Sudakov factor, however, falls fast enough as $Q_{t} \rightarrow 0$ that it ensures the integral converges.

Combining all of these elements together, the full differential cross section for central exclusive production of a system $\Gamma$ may be written: 


$$
\hat{s} \frac{\partial}{\partial \hat{s} \partial y \partial t_{1} \partial t_{2}} \sigma(h h \rightarrow h+\Gamma+h)=\mathcal{S} \exp \left[b\left(t_{1}+t_{2}\right)\right] \hat{s} \frac{\partial \mathcal{L}}{\partial \hat{s} \partial y} \hat{\sigma}(g g \rightarrow \Gamma)
$$

where the $t$ dependence of the cross section is approximately

$$
\frac{\partial \sigma}{\partial t_{i}} \simeq \exp \left(b t_{i}\right)
$$

with $b \simeq 4 \mathrm{GeV}^{2}$. The cross section for gluon fusion to $\Gamma$ is $\hat{\sigma}$ and $\Gamma$ must be averaged in the amplitude over incoming the gluon colours and polarisations, as in equation 2.36. The differential luminosity, $\hat{s} \frac{\partial \mathcal{L}}{\partial \hat{s} \partial y}$, is given by

$$
\hat{s} \frac{\partial \mathcal{L}}{\partial \hat{s} \partial y}=\left(\frac{\pi}{8} \int \frac{d Q_{t}^{2}}{Q_{t}^{4}} f_{g}\left(x_{1}, Q_{t}, \mu\right) f_{g}\left(x_{2}, Q_{t}, \mu\right)\right)^{2}
$$

where the off-diagonal un-integrated gluon distribution functions $f_{g}$ are approximately:

$$
f_{g}\left(x_{1}, Q_{t}, \mu\right) \simeq R_{g} \frac{\partial}{\partial \ln Q_{T}^{2}}\left(\sqrt{T\left(Q_{t}, \mu\right)} x g\left(x_{1}, Q_{t}\right)\right)
$$

where $R_{g}$ is the factor that accounts for the screening gluon having different longitudinal momentum fraction. The factor $\mathcal{S}$ in equation 2.40 is the soft survival factor and is present in all diffractive cross sections. Further interactions between the two proton lines may produce additional emissions that make the event appear to be non-difractive. A certain proportion of diffractive events are therefore "lost" and the observed diffractive cross section is lower than the predicted cross section. The soft survival factor accounts for this difference between the predicted and observed cross sections.

The small size of the differential luminosity reduces the cross section compared to non-diffractive production of the same system $\Gamma$. Exclusive production conveys three major benefits, however, which make it worthwhile pursuing the smaller cross section. The first of these is that, for central exclusive production, there is no activity in a particle physics detector other than the system of interest, $\Gamma$. Contrast that with non-diffractive production in which the soft underlying 
event fills the detector with the remnants of the hadrons. Even diffractive production through Pomeron or Reggeon exchange results in Pomeron or Reggeon remnants populating the detector. Note, however, that at the LHC it is expected that, on average, around twenty proton collisions will occur per bunch crossing.

The second benefit of central exclusive production is that by installing proton tagging detectors along the beam line a large distance from the central detector, the outgoing and intact protons may be observed. By measuring the protons' energies and transverse momenta, and knowing the collision energy, it is possible to infer the so-called "missing mass" [5] in the central detector. The FP420 $[29,30]$ proposal at the LHC boasts of a possible $1 \mathrm{GeV}$ resolution on the mass of the central system. Not only could this be useful in measuring the masses of resonances such as the Higgs boson, but it could also be used to calibrate the observed jet energies to the actual jet energies. Further, because the tagged outgoing protons remain intact, it is known that the central system must possess zero angular momentum in the $z$ (beam-line) direction. Constraining the spin quantum numbers of any newly discovered particles at the LHC will be an important task, which might otherwise require a linear collider of polarised lepton beams.

The final advantage of central exclusive production is that the $J_{z}=0$ "spin selection rule" suppresses the QCD background to many processes. For example, the dominant background to the Standard Model $H \rightarrow b \bar{b}$ channel is direct production of a pair of $b$ quarks. Ordinarily, this background is huge and results in the $H \rightarrow b \bar{b}$ channel being unfavoured as the discovery channel. However, in central exclusive production the two $b$ quarks must be back to back and must have the same helicities due to the $J_{z}=0$ selection rule. There is destructive interference in the leading order amplitude between the two possible spin configurations and in the limit that the $b$ quark mass is zero the cross section is also zero. The full suppression of the leading order cross section is $m_{b}^{2} / \hat{s}$, so if $\sqrt{\hat{s}} \simeq 100 \mathrm{GeV}$ for the Standard Model Higgs boson then the cross section for the QCD background is suppressed by a factor of less than 0.002. Despite the much lower production cross section in central exclusive production, the signal 
to background ratio may actually be more favourable. The QCD background is not suppressed at the next to leading order (NLO), in which there is additional gluon radiation from a quark line and it is therefore important to take the NLO contribution to the cross section into account. 


\section{Chapter 3}

\section{Exotic uses for Central Exclusive Production}

\subsection{A CP Violating Supersymmetric Higgs Boson}

There is an excess of matter over anti-matter in the Universe of the order of 1 part in $10^{9}$, which means that for every matter particle there are approximately a billion photons in the Universe today. This is usually explained by positing the existence of $\mathrm{CP}$ violating interactions that occur at different rates for particles and anti-particles.

The amount of $C P$ violation present in the CKM matrix of the Standard Model is nowhere near enough to account for the amount of matter present in the Universe [31]. A potential source of $C P$ violation that is not present in the Standard Model lies in the Higgs sector of the MSSM. The MSSM requires the existence of two Higgs doublets, $H_{u}$ to generate a mass for the up type quarks and $H_{d}$ to generate a mass for the down type quarks. The two Higgs doublets are written

$$
H_{u}=\left(\begin{array}{c}
\phi_{1}^{+} \\
\frac{1}{\sqrt{2}}\left(\phi_{1}+i a_{1}+v_{u}\right)
\end{array}\right), H_{d}=\left(\begin{array}{c}
\phi_{2}^{+} \\
\frac{1}{\sqrt{2}}\left(\phi_{2}+i a_{2}+v_{d}\right)
\end{array}\right)
$$


where $\phi_{1,2}^{ \pm}$are the 4 charged degrees of freedom, $\phi_{1,2}$ are the uncharged $C P$ even degrees of freedom, $a_{1,2}$ are two uncharged $C P$ odd degrees of freedom and $v_{u, d}$ are the vacuum expectation values (VEVs) of the Higgs doublets, whose ratio is written as

$$
\frac{v_{u}}{v_{d}}=\tan (\beta) .
$$

As in the Standard model, two of the charged degrees of freedom get "eaten" by the longitudinal polarisation of the $W^{ \pm}$bosons and one of the $C P$ odd degrees of freedom gets eaten by the $Z^{0}$ boson to leave two charged physical Higgs bosons, two un-charged $C P$ even degrees of freedom and an un-charged $C P$ odd degree of freedom. The physical $C P$ odd degree of freedom is a mixture of the $a_{1}$ and $a_{2}$ fields:

$$
a=a_{1} \sin (\beta)+a_{2} \cos (\beta)
$$

It is possible that squark loops in the Higgs boson propagator can produce mixing between the $C P$ even $\phi_{1,2}$ and the $C P$ odd $a$ in the mass eigenstates of the Higgs fields. The resulting mass eigenstate is therefore not an eigenstate of the $C P$ operator and $C P$ violation is introduced into the Higgs sector. The mixing will be produced if there is a phase difference between the $\mu$ term of the MSSM superpotential, which produces the tree-level mass for the Higgs fields (the last term on the right of equation 3.6), and the trilinear SUSY breaking coupling in the MSSM Lagrangian, which provide loop corrections to the Higgs bosons propagators. Figure 3.1 shows the mixing between the $\phi_{1}$ and $a$ via a sfermion loop. One might think that the loop diagram of figure 3.1 would violate $C P$ even if the phase between the two diagrams were zero. However, if $|+\rangle$ is a $C P$ even eigenstate and $|-\rangle$ is a $C P$ odd eigenstate then $| \pm\rangle$, the superposition of the two, may also be an eigenstate of $C P$ :

$$
| \pm\rangle=C_{1}|+\rangle+i C_{2}|-\rangle
$$




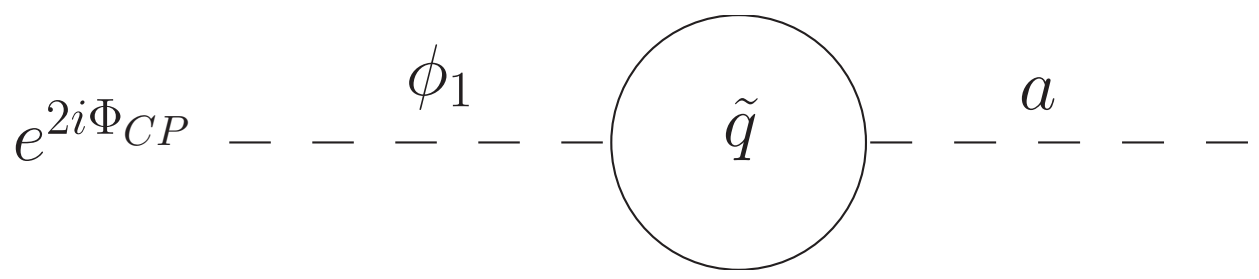

Figure 3.1: The Higgs mass eigenstates are a mixture of all three weak eigenstates due to mixing between the tree level $\mu$ term and through a coupling to the squarks at the one loop level.

$$
\begin{aligned}
C P| \pm\rangle & =C_{1} C P|+\rangle-i C_{2} C P|-\rangle \\
& =| \pm\rangle
\end{aligned}
$$

where $C_{1}$ and $C_{2}$ are the Clebsch-Gordon coefficients such that $C_{1}^{2}+C_{2}^{2}=1$. Physically, equation 3.4 means that the rate at which $\phi_{1} \rightarrow a$ due to the loop in the propagator of figure 3.1 is exactly the same as the rate at which $a \rightarrow \phi_{1}$, hence there is no overall $C P$ violation in the absence of a phase difference. If there is a phase difference between the two diagrams, however, then the propagating eigenstate is not an eigenstate of $C P$ and $C P$ violation results. The orthogonal matrix that rotates between the weak $(C P)$ and mass eigenstates of the Higgs bosons is $O$ :

$$
\left(\begin{array}{c}
H_{1} \\
H_{2} \\
H_{3}
\end{array}\right)=O\left(\begin{array}{c}
\phi_{1} \\
\phi_{2} \\
a
\end{array}\right)
$$

The CPX scenario [6] of the MSSM was designed to show off the effects of CP violation in the Higgs sector of the MSSM. The CPX scenario introduces a complex phase between the $\mu$ term coupling the two Higgs superfields and the trilinear Yukawa coupling between the sfermions and the Higgs. The superpotential for the Higgs sector of the CPX scenario is [32,33]:

$$
\Delta W_{C P X}=h_{l} \hat{H}_{d}^{T} i \tau_{2} \hat{L} \hat{E}+h_{d} \hat{H}_{d}^{T} i \tau_{2} \hat{Q} \hat{D}+h_{u} \hat{Q}^{T} i \tau_{2} \hat{H}_{2} \hat{U}-\mu \hat{H}_{d}^{T} i \tau_{2} \hat{H}_{u}
$$


where $\hat{H}_{u}$ and $\hat{H}_{d}$ are the Higgs superfields, $\hat{L}$ and $\hat{E}$ are the right and left-handed leptonic superfields and $\hat{U}, \hat{D}$ and $\hat{Q}$ are the superfields of the right-handed up and down quarks and the left-handed quark $S U(2)$ doublet. The second Pauli matrix is denoted by $\tau_{2}$. Those soft SUSY breaking terms in the MSSM Lagrangian that, in conjunction with the $\mu$ term of equation 3.6, produce the $\mathrm{CP}$ violation are $[32,33]$ :

$$
\Delta \mathcal{L}_{C P X}=A_{l} \Phi_{1}^{\dagger} \tilde{L} \tilde{E}+A_{d} \Phi_{1}^{\dagger} \tilde{Q} \tilde{D}-A_{u} \Phi_{2}^{T} i \tau_{2} \tilde{Q} \tilde{U}+\text { h.c. }
$$

where $\tilde{L}, \tilde{E}, \tilde{Q}, \tilde{U}$ and $\tilde{D}$ are the sleptons and squark fields, $A_{l}, A_{d}$ and $A_{u}$ are trilinear couplings, $\Phi_{1,2}$ are the Higgs bosons.

The masses and couplings defined in the CPX scenarios are as follows: a universal mass of $500 \mathrm{GeV}$ for the third generation squarks, $\tilde{M}_{t, b}$, and the SUSY breaking mass $M_{S U S Y}$; a universal trilinear coupling $A$ of magnitude $|A|=1 \mathrm{TeV}$; a $\mu$ parameter of $2 \mathrm{TeV}$; a gluino mass of $1 \mathrm{TeV}$ and a mass of $200 \mathrm{GeV}$ for the winos and the bino. The $C P$ violation occurs at the one loop level in the Higgs propagator (figure 3.1). The $C P$ violating phase, $\Phi_{C P}$, is a phase difference between $\mu$ and $A$, that is, if $\mu$ is real then $A=e^{2 i \Phi_{C}}|A|$.

The Higgstrahlung search channel at LEP looked for Higgs radiated from a Z boson:

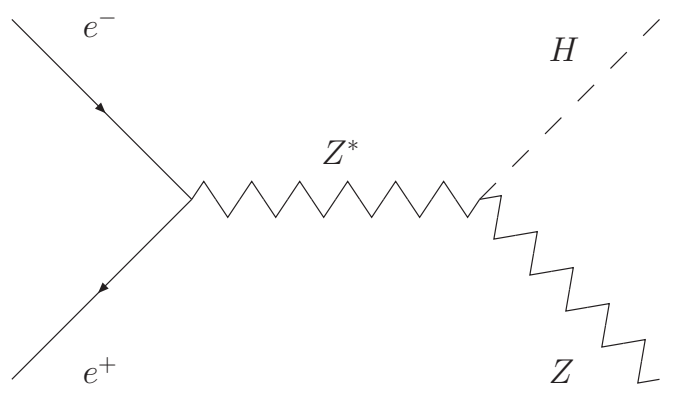

Figure 3.2: Higgstrahlung at the LEP collider.

If the lightest Higgs boson contains a significant contribution from the $C P$ odd $a$ field then its coupling to the $Z$ boson will be suppressed, reducing the rate at which figure 3.2 occurs. It is not possible to suppress the couplings of all three Higgs bosons to the $Z$, since the sum of the three Higgs- $Z$ couplings, $g_{H_{i} Z}$ ， 
obeys the rule [34]:

$$
\sum_{i} g_{H_{i} Z}^{2}=g_{H_{S . M .} Z}^{2}
$$

where $g_{H_{S . M .} Z}$ is the standard model Higgs- $Z Z$ vertex coupling. However, if $H_{2}$ and $H_{3}$ can both decay to a pair of $H_{1}$ then the searches employed at LEP, which anticipated four jets at most, will have failed to find the six jets that result from $H_{2,3}$ production and the $H_{1}$ production rate is too low to be observed due to the suppressed coupling. In this case, all three MSSM Higgs bosons under the CPX scenario would have avoided detection at LEP, even if the lightest Higgs mass were well below the $114 \mathrm{GeV}$ limit placed on the Standard Model Higgs. This is illustrated in figure 3.3 taken from [34], which shows un-probed regions in the top two panes below $\tan \beta=5$ in which the lightest MSSM Higgs boson has a mass below $40 \mathrm{GeV}$. 


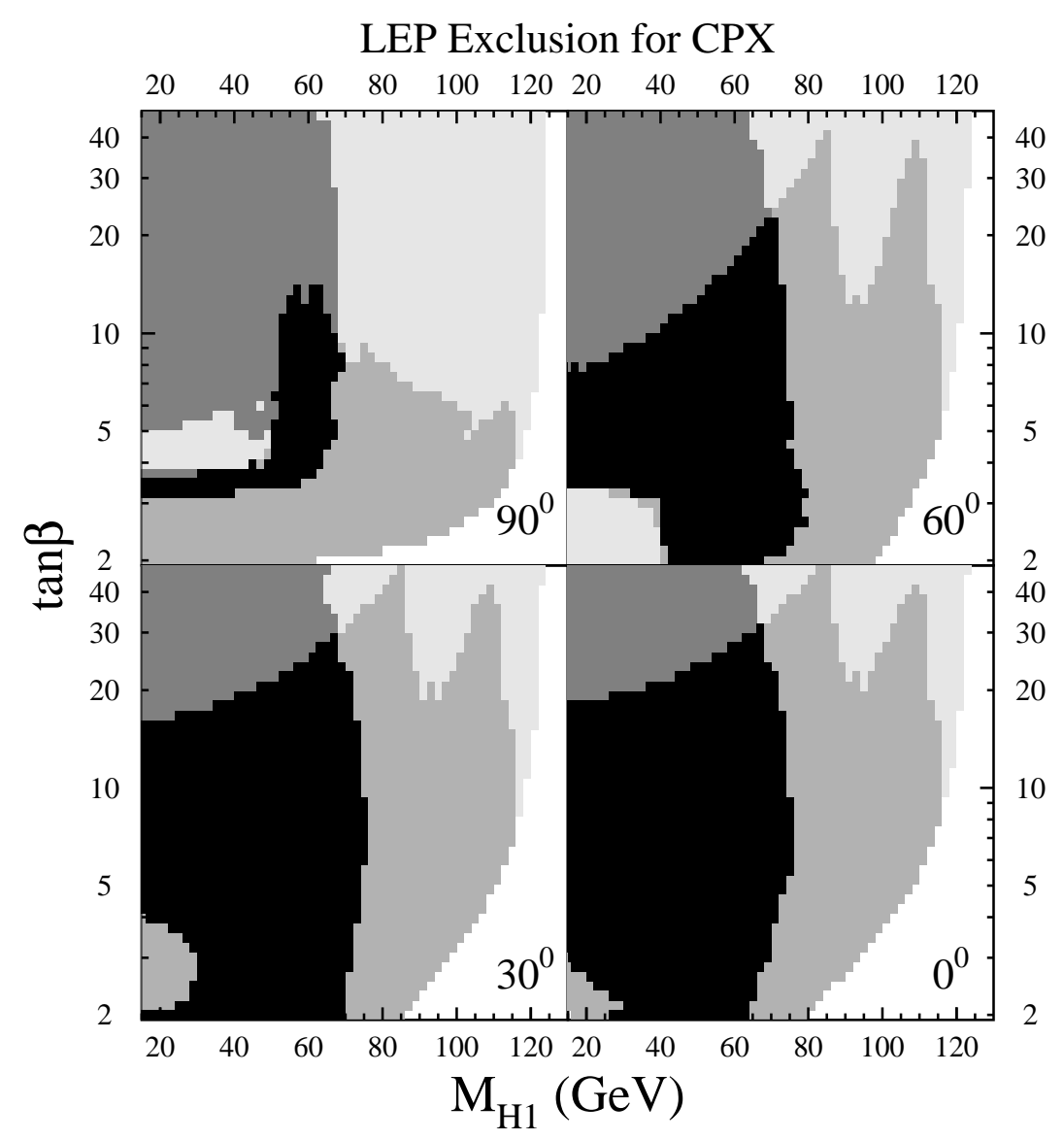

Figure 3.3: Regions of MSSM parameter space excluded by LEP in the CPX scenario [34]. Four Different $C P$ violating phases $\Phi_{C P}$ are shown under the CPX scenario. The white region is inconsistent with electroweak symmetry breaking, the medium grey is excluded by Higgstrahlung (figure 3.2), the dark grey is excluded by $e^{+} e^{-} \rightarrow Z^{*} \rightarrow H_{i} H_{j}$ and the black region is the overlap between both searches. Significantly, there are regions, shown in the lightest shade of grey, that are not covered by either search.

By far the dominant decay mode of such a light Higgs boson would be $H \rightarrow b \bar{b}$. It would be extremely difficult to detect this peak in the $b \bar{b}$ jet production cross section at the Tevatron and even more so at the LHC, in both cases due to the large QCD background of b-jets (and falsely identified b-jets). It would seem then that in such a scenario the MSSM Higgs sector is extremely difficult to observe [34].

Central exclusive production of the lightest MSSM Higgs boson now shows its usefulness. The QCD background is significantly reduced by the $J_{z}=0$ spin 


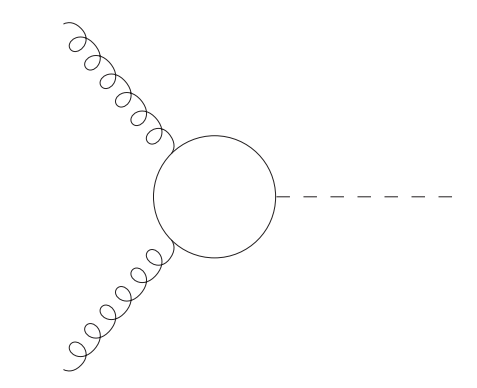

Figure 3.4: Higgs boson production via gluon fusion.

selection rule, the absence of underlying event makes jet measurement easier and, potentially, the outgoing proton taggers provide an accurate measurement of the central mass.

\subsubsection{Cross Section Calculation for CPX Higgs Boson Production}

In order to calculate the cross section for a centrally produced CPX Higgs boson one first needs the cross section for gluon fusion to a CPX Higgs, by way of heavy quark or squark loops (figure 3.4).

The amplitude for $g g \rightarrow H_{1}$ is $[35,36]:$

$$
\begin{array}{r}
\mathcal{M}_{\epsilon_{1} \epsilon_{2}}^{a b}=\frac{m_{H_{1}} \alpha_{s}\left(m_{H_{1}}\right) \delta^{a b}}{4 \pi}\left\{S_{1}^{g}\left(m_{H_{1}}\right)\left(\epsilon_{1 \mu} \epsilon_{2}^{\mu}-2 \frac{k_{1}^{\mu} \epsilon_{2 \mu} k_{2}^{\nu} \epsilon_{1 \nu}}{m_{H_{1}}^{2}}\right)\right. \\
\left.-2 P_{1}^{g}\left(m_{H_{1}}\right) \frac{\epsilon_{\mu \nu \rho \lambda} \epsilon_{1}^{\mu} \epsilon_{2}^{\nu} k_{1}^{\rho} k_{2}^{\lambda}}{m_{H_{1}}^{2}}\right\}
\end{array}
$$

where $k_{1,2}$ and $\epsilon_{1,2}$ are the momenta and polarisations of the incoming gluons, $a, b$ are the colours of the incoming gluons, $m_{H_{1}}$ is the mass of the Higgs boson and $S_{1}^{g}$ and $P_{1}^{g}$ are the 1-loop scalar and pseudo-scalar form factors for a pair of gluons to couple to the $H_{1}$ boson. In the limit that the fusing gluons have the same polarisation, as is necessary for central exclusive production, the term $\epsilon_{\mu \nu \rho \lambda} \epsilon_{1}^{\mu} \epsilon_{2}^{\nu}$ vanishes and, additionally, since the gluons are approximately collinear the term $k_{1}^{\mu} \epsilon_{2 \mu} k_{2}^{\nu} \epsilon_{1 \nu}$ also disappears. The scalar form factor $S_{1}^{g}$ is given by [37]: 


$$
S_{1}^{g}\left(m_{H_{1}}\right)=\sum_{f} g_{s f}^{1} \frac{m_{H_{1}}}{m_{f}} F_{s f}\left(\tau_{f}\right)+\frac{1}{4} \sum_{\tilde{f}} \sum_{i=1,2} g_{\tilde{f}}^{1} \frac{m_{H_{1}}}{m_{\tilde{f}}^{2}} F_{0}\left(\tau_{\tilde{f}}\right)
$$

where the sums over $f$ and $\tilde{f}$ are over all quark and squark flavours respectively with masses $m_{f}$ and $m_{\tilde{f}}$. The additional sum over $i$ in the second term accounts for the fact that, since a spin 1/2 fermion has twice as many degrees of freedom as a spin 0 sfermion, there are two squarks for every quark flavour. The couplings $g_{\tilde{f}}^{1}$ and $g_{s f}^{1}$ are of the $H_{1}$ boson to the squark current and the scalar quark current (as opposed to the pseudo-scalar current, which is not invariant under the parity operator). The auxiliary functions $F_{s f}$ and $F_{0}$ arise from the loop integral in figure 3.4 and are

$$
\begin{gathered}
F_{\text {sf }}(\tau)=\tau^{-1}\left(1+\left(1-\tau^{-1}\right) f(\tau)\right) \\
F_{0}(\tau)=\tau^{-1}\left(-1+\tau^{-1} f(\tau)\right) \\
\left\{\begin{array}{cc}
\arcsin ^{2}(\sqrt{\tau}) & \tau \leq 1 \\
-\frac{1}{4}\left(\ln \left(\frac{1+\sqrt{1-\tau^{-1}}}{1-\sqrt{1-\tau^{-1}}}\right)-i \pi\right)^{2} & \tau>1
\end{array}\right.
\end{gathered}
$$

where $\tau=m_{H_{1}}^{2} / 4 m_{f}^{2}$. The coupling $g_{s f}^{1}$ is given by the terms in the MSSM Lagrangian that couple fermionic currents to the Higgs bosons $H_{i}$, which are [36]:

$$
\Delta \mathcal{L}_{H f}=-\frac{g m_{f}}{2 M_{W}} \bar{f}\left(\frac{v_{f}^{i}}{R_{\beta}^{f}}-i \frac{\bar{R}_{\beta}^{f} \alpha_{f}^{i}}{R_{\beta}^{f}} \gamma_{5}\right) f H_{i}+\text { h.c. }
$$

where $g$ is the electroweak coupling constant and $M_{W}$ is the mass of the $W$ boson. Note that the Standard Model Higgs-fermion coupling is $g m_{f} / 2 M_{W}$. The terms $v_{f}^{i}$ and $\alpha_{f}^{i}$ are elements from the matrix that rotates between the mass and weak eigenstates of the three Higgs bosons. $v_{f}^{i}$ gives the $\phi_{1}$ (if $f$ is a up-type quark) or $\phi_{2}$ (if $f$ is an down-type quark) content of the $i^{\text {th }}$ physical Higgs boson, while $\alpha_{f}^{i}$ gives the $a$ content. $R_{\beta}^{f}$ is $\cos (\beta)$ for down type quarks or $\sin (\beta)$ for up type quarks and accounts for the different VEVs of the two Higgs doublets. The term in $\gamma_{5}$ couples to the pseudo-scalar current and is not needed to first order in central exclusive production because it produces a helicity flip in the quark line 
at the $H q q$ vertex, which is not allowed due to the $J_{z}=0$ selection rule. The scalar coupling $g_{s f}^{1}$ is therefore given by

$$
g_{s f}^{1}=\frac{g m_{f}}{2 M_{W}} \frac{v_{f}^{1}}{R_{\beta}^{f}}
$$

The couplings of the chiral states of the squarks to the weak eigenstates of the Higgs bosons are given in [35]. These couplings must be rotated in order to give the couplings of the mass eigenstates of the Higgs bosons to the mass eigenstates of the squarks. For example, the coupling of the Higgs field $\phi_{1}$ to the $\tilde{t}_{1}$ and $\tilde{t}_{2}$ stop squark fields (two stop squarks are necessary to match the two possible spin states of the top quark) is given by $\tilde{C}_{\phi_{1} ; \tilde{t}_{1} ; \tilde{t}_{2}}^{t}$ :

$$
\tilde{C}_{\phi_{1} ; \tilde{t}_{1} ; \tilde{t}_{2}}^{t}=\frac{g m_{t}}{2 m_{W} \sin \beta}|\mu| e^{i \Phi_{C P} A \mu}
$$

where $m_{t}$ is the mass of the top quark. The couplings of the mass eigenstate of the Higgs to the mass eigenstates, $\tilde{t}_{m_{\{1,2\}}}$ of the stop squark are therefore given by $\tilde{C}_{h_{i} ; \tilde{t}_{m_{j}} ; \tilde{t}_{m_{k}}}$ :

$$
\tilde{C}_{h_{i} ; \tilde{t}_{m_{j}} ; \tilde{t}_{m_{k}}}=O_{a, i} \tilde{C}_{\phi_{a} ; \tilde{t}_{b} ; \tilde{t}_{c}}^{t} U_{j, b}^{\dagger} U_{c, k} .
$$

where $O_{a, i}$ is the mixing matrix between the $a^{t h}$ Higgs field in the weak basis (including the $C P$ odd $a$ ) and the $i^{\text {th }}$ Higgs mass eigenstate, $\phi_{a}$ are the three weak eigenstates of the Higgs fields and $U_{c, k}$ is the mixing matrix between the $k^{\text {th }}$ mass and $c^{\text {th }}$ chiral eigenstates of the $\tilde{t}$. There is an implicit sum over $a, b$ and $c$.

The computer program $\mathrm{CPH}+[33,38]$ was used to provide all of the necessary MSSM couplings in the weak and chiral basis, as well as the masses of the MSSM particles. $\mathrm{CPH}+$ was additionally modified to provide the rotation matrices from the weak and chiral states of the Higgs and squarks to the propagating mass states.

The cross section for the hard sub-process, after averaging over the gluon colours and polarisations, and assuming a narrow width for the Higgs boson is: 


$$
\hat{\sigma}_{g g \rightarrow H_{1}}(\hat{s})=\frac{\alpha_{s}(\hat{s})^{2}}{16 \pi}\left|S_{g}^{1}(\hat{s})\right|^{2} \delta\left(1-\frac{m_{H_{1}}^{2}}{\hat{s}}\right)
$$

When convoluted with the differential luminosity of equation 2.41 and integrated over $t_{1,2}$ and $x_{1,2}$, equation 3.16 gives the cross section for central exclusive production of the lightest Higgs boson in the CPX scenario.

\subsubsection{Results for Central Production of a CP Violating Higgs Boson}

The cross section for central exclusive production of the lightest $C P$ violating MSSM Higgs boson is shown in figure 3.5 [39] for both the Tevatron and the LHC. Values for $\tan (\beta)$ of 4 and 5 were used, with a $C P$ violating phase of $\Phi_{C P}=90^{\circ}$ in order that the region left un-probed by LEP in figure 3.3 could be explored. The MRST 2002 [23] PDF set was used to provide the integrated gluon distributions for the proton, from which the un-integrated distributions were estimated using equation 2.42. A fixed $R_{g}$ factor that accounts for the skewed gluon distributions of 1.2 was used at the LHC and 1.4 at the Tevatron. A constant soft survival factor of 0.02 (LHC) or 0.045 (Tevatron) $[2,40]$ was used. The cross section was integrated over all $t_{1,2}$ and over $x_{1,2} \leq 0.1$. The ratio of the scalar form factor $S_{g}^{1}$ in the CPX scenario to that in the Standard Model is shown in figure 3.6, which shows the contribution to the scalar form factor from bottom quark loops only, bottom and top quark loops and bottom, top and stop loops. Figures 3.5 and 3.6 are discussed in more detail in section 3.2.3.

\subsection{Warped Extra Dimensions}

The hierarchy problem is essentially caused by the weakness of gravity in relation to the other fundamental forces; the small value of Newton's constant $G_{N}$ implies a very large scale; the Planck mass of $M_{p}=1 / \sqrt{8 \pi G_{N}}$. Theories with extra dimensions solve the hierarchy problem by allowing gravity access to the volume of the extra dimension(s) - called the bulk - while restricting the Standard Model forces and particles to a four-dimensional slice of space-time called the brane. The brane forces then appear much stronger than gravity not because the coupling 

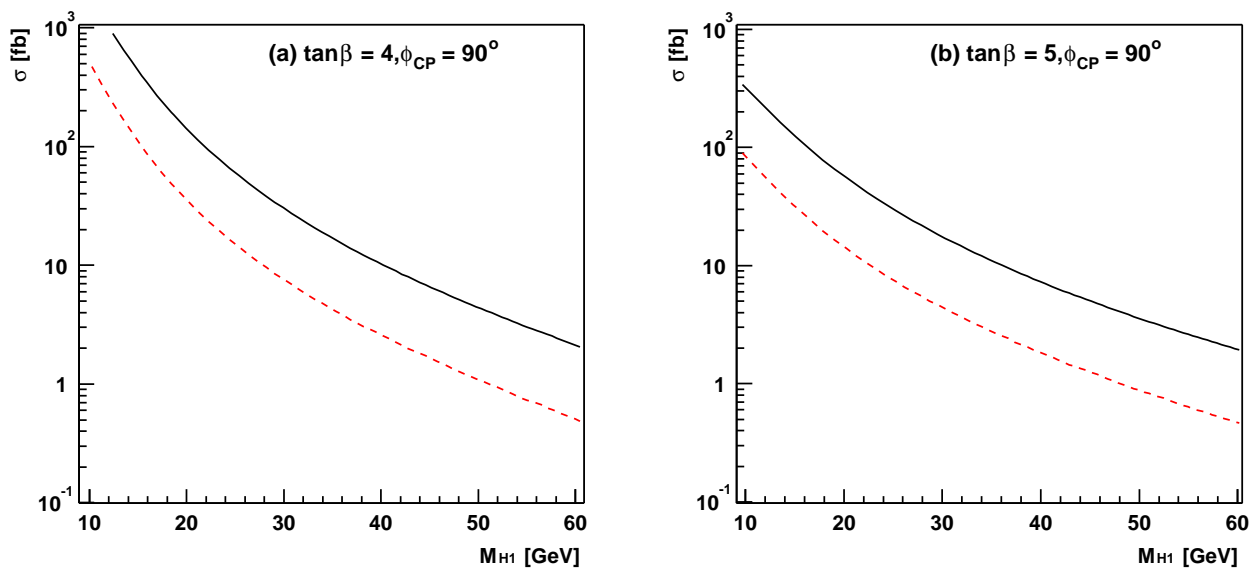

Figure 3.5: The cross section for central exclusive production of the lightest $\mathrm{CP}$ violating MSSM Higgs boson at the Tevatron (red dashed curve) and LHC (solid) [39].
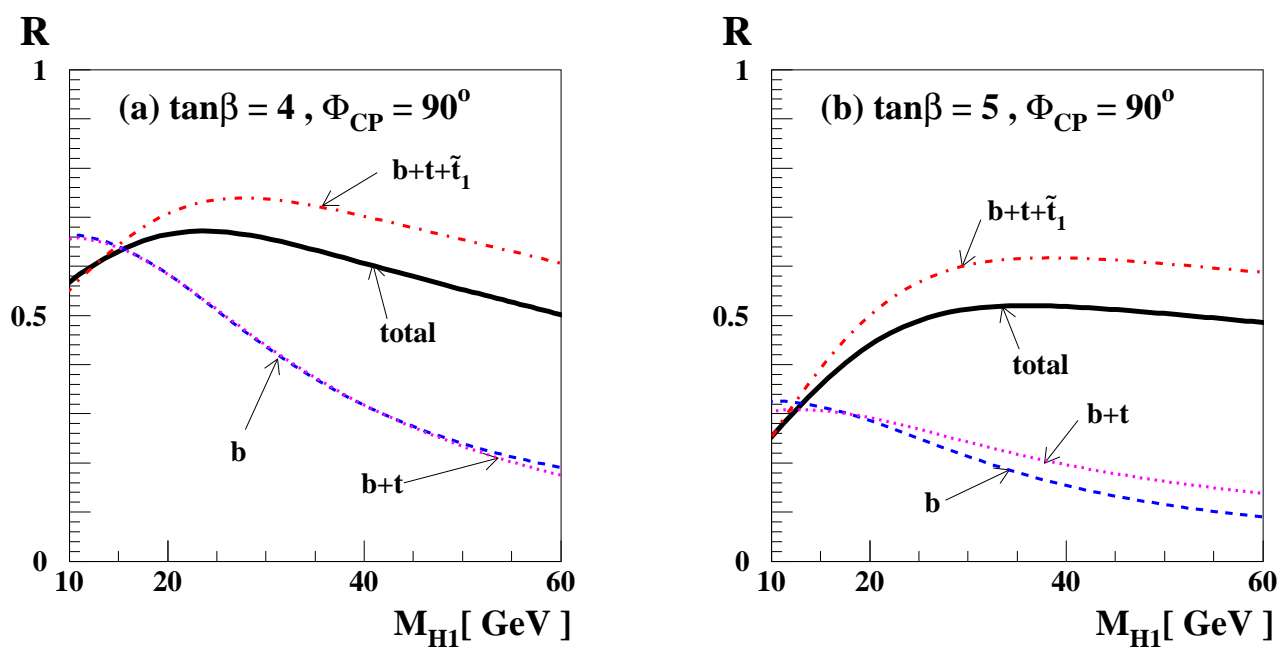

Figure 3.6: The ratio $R=\left|S_{g}^{1}\left(m_{H_{1}}^{2}\right) / S_{S M}\left(m_{H_{S M}}\right)\right|$ with $m_{H_{1}}=m_{H_{S M}}$ in the CPX scenario with $M_{S U S Y}=0.5 \mathrm{TeV}, \Phi_{C P}=\pi / 2$ and $\tan (\beta)=4$ or 5 [39]. 
constants are hugely different, but because gravity is radiating into the volume of the bulk and the Planck scale as observed on the brane is enhanced by a factor of $V_{B}$, the volume of the bulk.

The Randall Sundrum model $[7,41]$ introduces a five-dimensional space-time using a metric with the following line element:

$$
d s^{2}=e^{-2 k y} d s^{\prime 2}-d y^{2}
$$

where $d s^{\prime 2}$ is the usual four-dimensional expression and $y$ is the additional coordinate in the bulk with a range of $\left\{0, r_{c} \pi\right\}$, the Standard Model living on a brane at $y=0$. An additional brane boundary to the bulk is introduced at $y=r_{c} \pi$, which produces a discrete spectrum of Kaluza-Klein excitations (as opposed to a continuous spectrum that would be obtained in the absence of a second brane). $r_{c}$ is a compactification radius and $k$ is a scale parameter which determines the extent to which the space-time in the bulk is warped. Any field of mass $m_{0}$ in the bulk will be rescaled on the Standard Model brane and will be observed to have a mass $m_{b r}$

$$
m_{b r}=m_{0} \exp \left(-k r_{c} \pi\right)
$$

So if $m_{0}$ is around the Planck mass, the natural scale in the bulk, masses on the brane will still be observed around the electroweak scale $(\mathcal{O}(100 \mathrm{GeV}))$ if $k r_{c} \sim 35$.

Along with gravitons on the Standard Model brane and their Kaluza Klein excitations, there is also the possibility for excitations of the separation between the two branes. The scalar field observed on the Standard Model brane as a result of such excitations is the radion [42], normalised as follows:

$$
\phi_{0}=\frac{e^{-k y_{0}}}{\sqrt{6} M_{p}} y(x)
$$

where $y_{0}$ is the expectation value of the separation of the two branes. Critically, the radion can mix with the kinetic energy terms for the Higgs field [43]: 


$$
\Delta \mathcal{L}=-\frac{1}{2}\left\{1+6 \gamma^{2} \xi\right\} \phi_{0} \square^{2} \phi_{0}-\frac{1}{2} \phi_{0} m_{r}^{2} \phi_{0}-\frac{1}{2} h_{0}\left(\square^{2}+m_{0}^{2}\right) h_{0}-6 \gamma \xi \phi_{0} \square^{2} h_{0}
$$

where $h_{0}$ is the Higgs field in five dimensions, $m_{r}$ and $m_{h}$ are mass terms for the radion and Higgs boson and $\gamma=v_{h} / \Lambda_{r}$, the ratio of the VEVs of the Higgs and radion fields. The first two terms in equation 3.20 are kinetic and mass terms for the radion, the second two are kinetic and mass terms for the Higgs boson and the last term in equation 3.20 produces the mixing between the fields, with $\xi$ the mixing parameter. The two mass eigenvalues are $[43,44]$ :

$$
m_{ \pm}^{2}=\frac{1}{2 Z^{2}}\left(m_{r}^{2}+\beta m_{h}^{2} \pm\left(\left[m_{r}^{2}+\beta m_{h}^{2}\right]^{2}-4 Z^{2} m_{r}^{2} m_{h}^{2}\right)^{1 / 2}\right)
$$

where $Z^{2}=1+6 \xi \gamma^{2}(1-6 \xi)$ and $\beta=1+6 \xi \gamma^{2}$. The mass eigenstates $h$ and $\phi$ are related to $h_{0}$ and $\phi_{0}$ by:

$$
\left(\begin{array}{l}
h_{0} \\
\phi_{0}
\end{array}\right)=\left(\begin{array}{ll}
d & c \\
b & a
\end{array}\right)\left(\begin{array}{l}
h \\
\phi
\end{array}\right)
$$

where $a, b, c$, and $d$ are:

$$
\begin{aligned}
a & =-\cos (\theta) / Z \\
b & =\sin (\theta) / Z \\
c & =\sin (\theta)+6 \xi \gamma \cos (\theta) / Z \\
d & =\cos (\theta)-6 \xi \gamma \sin (\theta) / Z
\end{aligned}
$$

where $\tan (2 \theta)=12 \gamma \xi Z m_{h}^{2} /\left(m_{\phi}^{2}-m_{h}^{2}\left(Z^{2}-36 \xi^{2} \gamma^{2}\right)\right)$.

The radion field $\phi_{0}$ does not couple to the $Z^{0}$ boson other than through its mixing with the Higgs field, which in turn reduces the coupling of the mass eigenstate of the Higgs boson to the $Z^{0}$ boson. Thus if $m_{+}$(equation 3.21) is above the scale probed by LEP and $m_{-}$has its coupling to the $Z^{0}$ boson 
suppressed through the mixing with the radion then both mass eigenstates may avoid detection.

\subsubsection{Cross Section Calculation for Central Exclusive Radion Produc- tion}

The hard sub-process cross section for $g g \rightarrow \phi_{r}$ is of the same form as equation 3.16, but with the scalar form factor for the MSSM Higgs boson replaced with the equivalent term for the radion. Using the Feynman rules given in [43, 45], the amplitude for production of a radion or Higgs boson by gluon fusion in the Randall-Sundrum model is:

$$
\mathcal{M}_{\epsilon_{1} \epsilon_{2}}^{a b}\left(g g \rightarrow \phi_{r}\right)=\frac{i \alpha_{s} \delta^{a b}}{4 \pi v}\left(k_{1} \cdot k_{2} g^{\mu \nu}-k_{1}^{\mu} k_{2}^{\nu}\right) \epsilon_{1 \nu} \epsilon_{2 \mu}\left[2 g_{f V} \sum_{q} F_{s f}\left(\tau_{q}\right)-14 g_{r}\right]
$$

where the summation over $q$ is of the quark flavours running round the loop, $v$ is the VEV of the Higgs boson and $g_{f V}$ is the coupling of the radion or Higgs field to the $Z^{0}$ boson, depending on which is to be produced. The term $g_{r}$ is produced by a coupling to the trace of the energy-momentum tensor for the gluons. The couplings $g_{f V}$ and $g_{r}$ are given by:

$$
g_{f V}=\left\{\begin{array}{l}
g(d+\gamma b) \\
g(c+\gamma a)
\end{array}, \quad g_{r}=\left\{\begin{array}{cc}
\gamma b & \text { Higgs } \\
\gamma a & \text { Radion }
\end{array}\right.\right.
$$

\subsubsection{Results for Central Exclusive Radion Production}

The parameters $g_{f V}$ and $g_{r}$ as well as the branching fractions and widths of the $\phi$ and $h$ were provided by Jai Sik Lee [46] as a function of the model parameters $\xi$, $\Lambda_{\phi}, m_{h}$ and $m_{\phi}$. The cross section equation 3.16 was then convoluted with the differential luminosity to give the cross section for central exclusive production of a radion or a Higgs at both the Tevatron and the LHC. The parameters pertinent to the differential luminosity calculation were identical with those of section 3.1.2. The cross section dependence on the Higgs boson and radion masses and the 

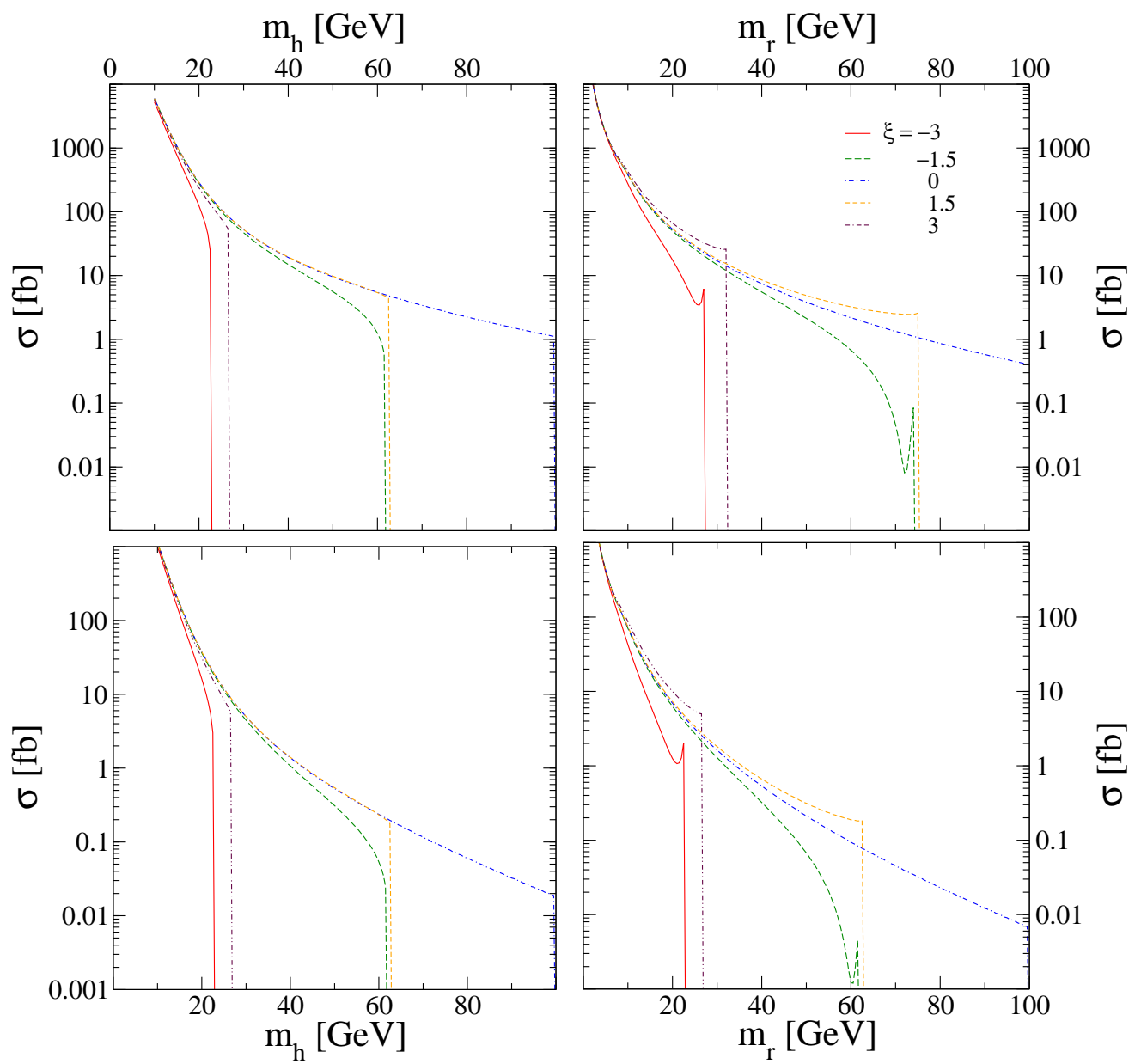

Figure 3.7: The production cross section for the Higgs boson (left column) and the radion (right column) in the Randall-Sundrum model at the LHC (top row) and Tevatron (bottom row). Curves are shown with mixing parameter $-3 \leq \xi \leq 3$.

mixing parameter is shown in figure 3.7. A VEV for the radion of $\Lambda_{\phi}=5 \mathrm{TeV}$ was used as in [43]. The cross section folded with the branching fraction for a $b \bar{b}$ pair is shown in figure 3.8 .

\subsubsection{Discussion}

The coupling of the Higgs boson to gluons by way of a squark or quark loop in the CPX scenario suffers from a suppression compared to the Standard Model Higgs boson (figure 3.6). The fact that the differential luminosity is very much higher for the CPX scenario due to its reduced Higgs boson mass, however, results in a similar sized cross section to a SM Higgs boson of $120 \mathrm{GeV}[2,47,48]$.

The cross sections for radion and Higgs production in figure 3.7 show a sudden turn off as the $m_{-}^{2}$ solution to equation 3.21 becomes negative. The dips 

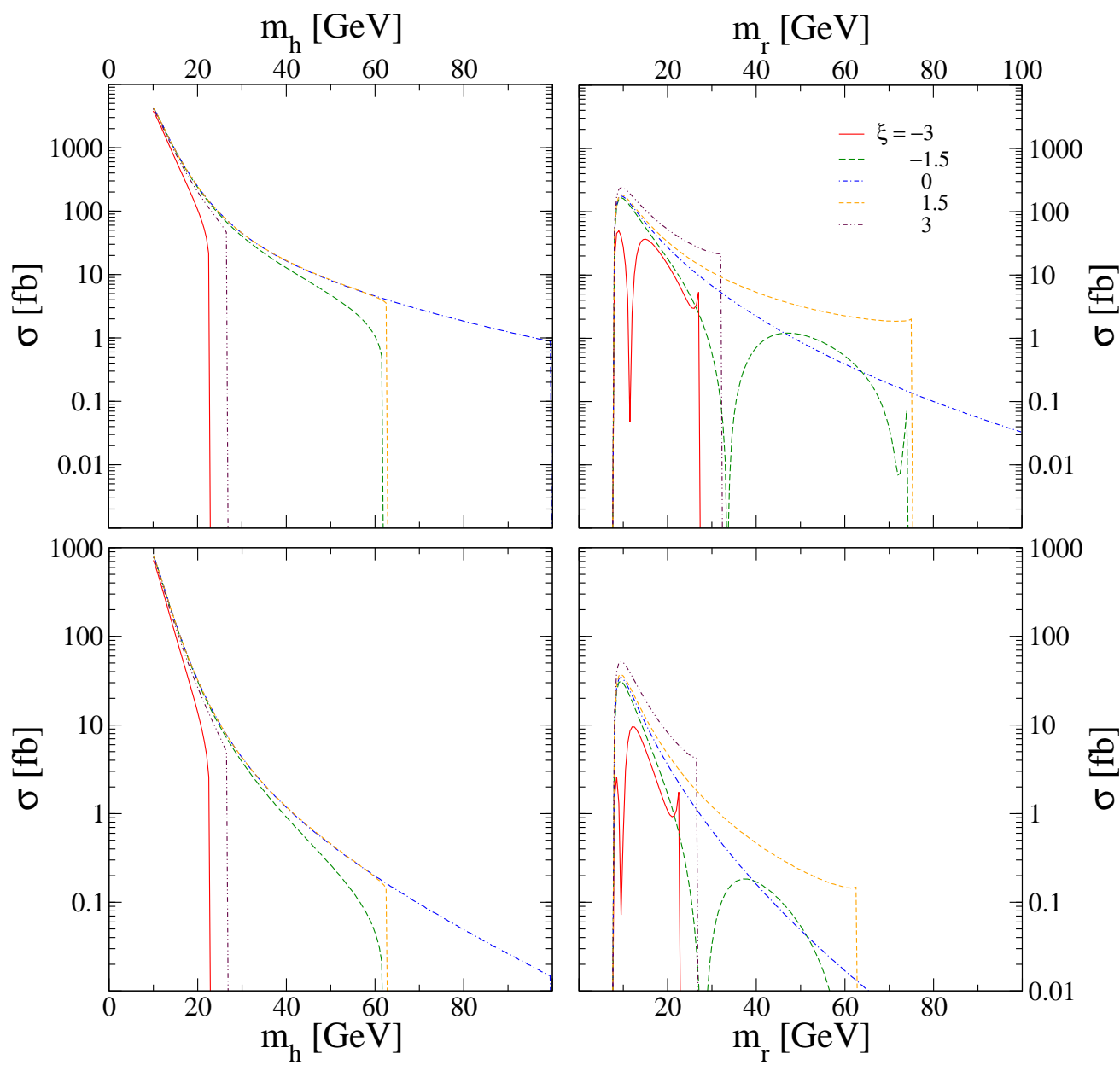

Figure 3.8: The central exclusive cross sections for $\phi \rightarrow b \bar{b}$ (right column) and $h \rightarrow b \bar{b}$ (left column) at the LHC (top row) and Tevatron (bottom row)

in the $\phi \rightarrow b \bar{b}$ cross section (figure 3.8) occur when there is cancellation between the Higgs-fermion and radion-fermion coupling in the physical radion-fermion coupling.

It initially appears that observation of either a radion or a $C P$ violating Higgs boson at the Tevatron may be possible, given a total integrated luminosity of around $5 \mathrm{fb}^{-1}$ and a cross section of between 1 and $10 \mathrm{fb}$ if the background truly is suppressed. However, the spin selection rule is weakened for the lighter states where the cross section is significant. The background is suppressed by a factor $m_{q}^{2} / m_{R^{\prime}}^{2}$, where $m_{q}$ is the mass of the quark and $m_{R}$ is the mass of the resonant state, hence the suppression is lower for lower mass states. Estimates of the leading order background to $120 \mathrm{GeV}$ Standard Model Higgs production at the LHC [48] give a signal to background ratio of order unity. Given that the Tevatron 
will only collect around $5 \mathrm{fb}^{-1}$ of integrated luminosity and the background for a $50 \mathrm{GeV}$ Higgs boson or radion is enhanced by a factor of around $120^{2} / 50^{2} \simeq 6$ relative to the Standard Model case, observation of either a radion or a $C P$ violating MSSM Higgs boson appears unlikely. Further, the signal to background ratio is proportional to the Higgs boson width $\Gamma(H \rightarrow g g)$, which is suppressed in the CPX scenario relative the Standard Model case (figure 3.6).

The case at the LHC shows more promise, both because of the increased production cross section and because of the vastly increased luminosity. However, as mentioned in section 2.4, the higher order contributions to the background may be significant. Jet finding and detector geometry may also have an impact. To carry out a more in-depth analysis of the physics potential of central exclusive production at the LHC, or indeed the Tevatron, a full simulation of both the signal and background is needed, which is the subject of the next chapter. 


\section{Chapter 4}

\section{The ExHuME Generator}

\subsection{Motivation}

A Monte Carlo simulation of a high energy physics collisions uses computer generated random numbers to calculate the total cross section for a process in a given kinematic range. Not only can the simulation calculate the cross section, but it can also reproduce the distributions of kinematical quantities, for instance the rapidity of the central system or the transverse momentum of the jets. A Monte Carlo simulation can therefore be extremely useful in understanding the distributions of final state particles from the hard process, the effect of kinematic cuts and the seperation of signal events from background events.

In this chapter the principle behind Monte Carlo integration will be explained, first with a simple example using the value of $\pi$ and then using the more specific case of ExHuME, which simulates the central exclusive production process. Finally, predictions from ExHuME for both the Tevatron and the Large Hadron Collider will be shown.

\subsection{Monte Carlo Integration}

The value of $\pi$ can be calculated by generating a sufficient number of random numbers. Consider a circle of radius 1 inside a square of side 2 centred on the origin (figure 4.1). The area of the square is 4 and $\pi$ is defined to be the area of 


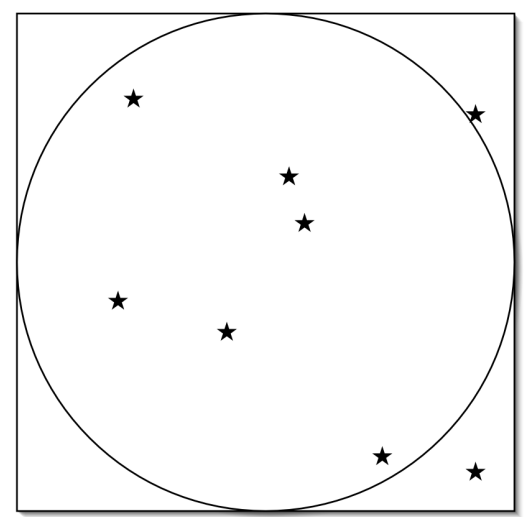

Figure 4.1: Calculating $\pi$ from random points within a square. Six of the eight points lie within the circle, so the estimate for $\pi$ from this limited sample of random numbers is 3 .

the circle. A set of $N$ points within the square are chosen by generating pairs of random numbers $\left\{x_{i}, y_{i}\right\}$ and the number of these points that also lie within the circle such that $x_{i}^{2}+y_{i}^{2}=1$ is $n$. Given a large enough $N$ the ratio $n / N$ should approach the ratio of the area of the circle to that of the square so the value of $\pi$ is given by

$$
\pi=\lim _{N \rightarrow \infty} 4 \times \frac{n}{N} .
$$

The method for calculating $\pi$, once generalised to higher dimension and with the function describing a circle replaced by a function describing the differential cross section, forms the basis for a Monte Carlo generator. In the case of the ExHuME generator the cross section is differential in at least 6 variables: the transverse momenta squared of the two outgoing protons, $t_{1}$ and $t_{2}$, the fractional longitudinal momentum losses of the two protons, $x_{1}$ and $x_{2}$, and the two azimuthal angles of the outgoing protons, $\phi_{1}$ and $\phi_{2}$. The $x_{1}$ and $x_{2}$ are simply related to the $\sqrt{\hat{s}}$ and $y$ and it is more convenient to supply those as input to the function that calculates the differential cross section. Depending on the gluon fusion sub-process there could be further variables, such as the opening angle between two centrally produced partons.

To generate an event the six variables are picked at random and the differential 
cross section calculated at that point in phase space. One further random number, $r$, is then required that should be in the range $\left\{0, \sigma_{\max }\right\}$, where $\sigma_{\max }$ is no lower than the maximum value of the differential cross section that it is possible to encounter. The value of $\sigma_{\max }$ can be determined either by prior knowledge of the shape of the differential cross section, for example the differential luminosity of central exclusive diffraction is always peaked at $y=0$, or by exploring the phase space before generating the events. The event will be accepted if $r$ lies below the calculated value of the differential cross section (figure 4.2). The fact that the differential luminosity is always peaked at $y=0$ is the reason why it is more convenient to use $\sqrt{\hat{s}}$ and $y$ instead of $x_{1,2}$ as input to the differential luminosity function.

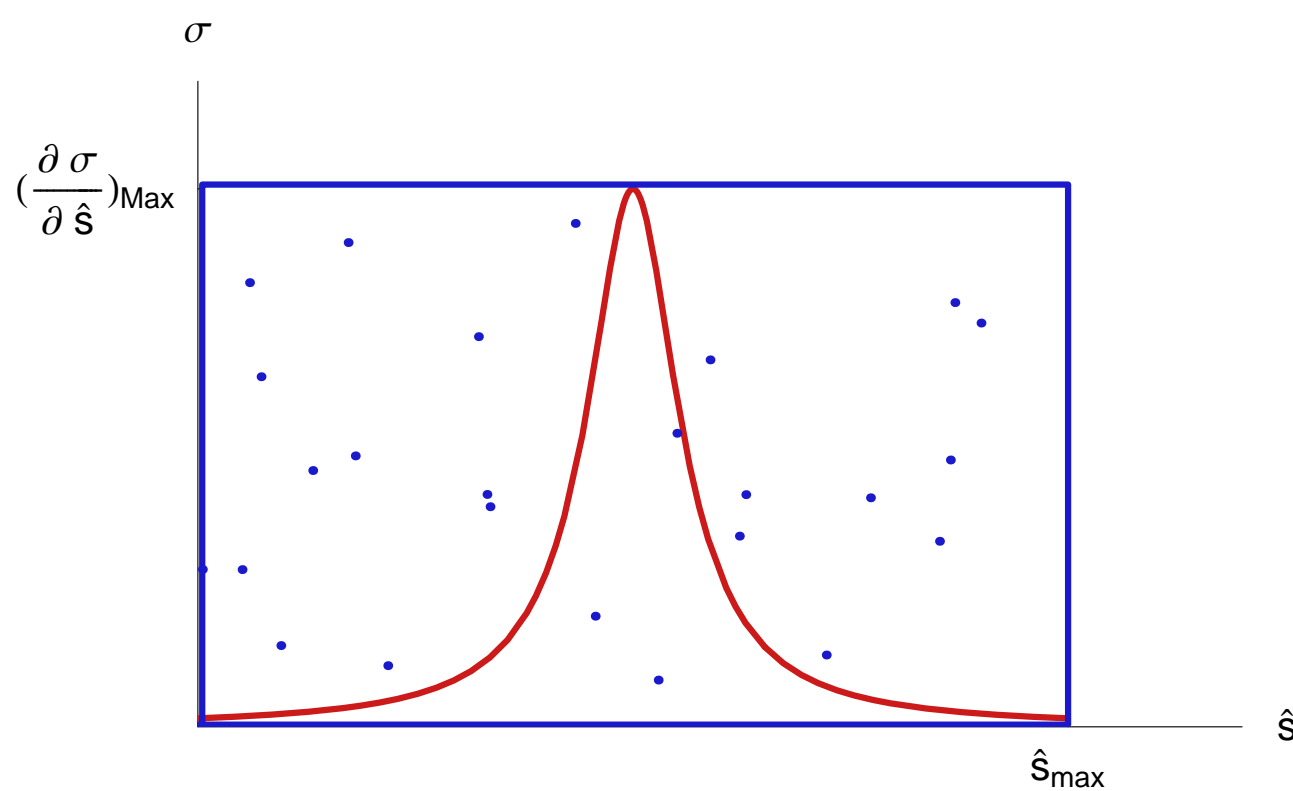

Figure 4.2: Using Monte Carlo techniques to integrate a differential cross section over the mass of the central system. There are 3 points out of 25 lying beneath the curve, so the cross section here is $\frac{3}{25} \times\left.\frac{\partial \sigma}{\partial \hat{s}}\right|_{\max } \times \hat{s}_{\max }$

In the full six-dimensional integration the value of the cross section is given by

$$
\sigma=\left.\frac{n}{N} \frac{\partial^{6} \sigma}{\prod_{i=1}^{6} \partial z_{i}}\right|_{\text {Max }} \prod_{i=1}^{6}\left(z_{\max }-z_{\min }\right)_{i}
$$


where $\partial \sigma /\left.\prod_{i=1}^{6} \partial z_{i}\right|_{\text {Max }}$ is the maximum value of the differential cross section, $z_{i}$ are the coordinates and $n$ events are accepted out of $N$ attempts to generate an event. The $n$ accepted events will produce the correct distributions of the $z_{i}$ for the given differential cross section.

The ExHuME simulator [10] takes advantage of the factorisation illustrated in figure 2.3 and the modularity available in the $\mathrm{C}++$ programming language. ExHuME provides a base cross section class, which contains the calculation of the differential luminosity (equation 2.41), holds the values of physical constants (such as particle masses and couplings) and keeps a record of the particle kinematics. An implementation of a CrossSection class should be passed to a class called Event, which handles the Monte Carlo generation and calculates the cross section as the final stage of the program. In this way, it is possible to access the functions pertinent to the cross section calculation completely independently of the event generation. For details on the usage of ExHuME see Appendix A.

\subsection{The Implementation of the Differential Luminosity}

The differential luminosity function is appropriate for either the LHC or the Tevatron, depending on the proton collision energy that is selected. The Sudakov factor $T\left(Q_{t}, \mu\right)$ (equation 2.39) is calculated by integrating numerically over $k_{t}$ using the implementation of Simpson's method available in the CERN libraries [49]. To leading order, the strong coupling constant, $\alpha_{s}\left(k_{t}\right)$, may be parameterised as

$$
\alpha_{s}\left(k_{t}\right)=\frac{12 \pi}{\left(33-2 N_{f}\right) \log \left(\frac{k_{t}^{2}}{\Lambda_{Q C D}^{2}}\right)}
$$

where $N_{f}$ is the number of quark flavours available and $\Lambda_{Q C D}$ is chosen such that $\alpha_{s}\left(M_{z}\right) \simeq 0.118$ ( $M_{z}$ is the mass of the $Z$ boson). For the hard scale a value of $\mu=0.618 \sqrt{\hat{s}}$ is used [50].

$T$ must be evaluated many times for each event because it lies within the numerical integration over $Q_{t}^{2}$ in the expression for the differential luminosity (equation 2.41). $T$ itself involves a numerical integration, which would result in 
the integrand in the exponent of equation 2.39 being evaluated $\mathcal{O}\left(10^{4}\right)$ times for each single evaluation of the differential luminosity. This is not prohibitive using a modern computer, but it is not very efficient and slows down the program a great deal. ExHuME avoids this speed penalty by caching the value of $T$ in a standard template library map indexed by $\mu$ each time that $T$ is calculated. When a subsequent call to $T(\mu)$ is made, ExHuME will check the map for entries that closely bracket the current value of $\mu$ and, if they exist, will interpolate the value of $T$ rather than carry out the integral. Curves showing the dependence of $T$ on $Q_{t}$ for $\sqrt{\hat{s}}$ ranging from $50 \mathrm{GeV}$ to $200 \mathrm{GeV}$ taken from ExHuME are shown in figure 4.3. As expected, the suppression is largest at small $Q_{t}$, although it is necessary to freeze the value of $\alpha_{s}$ to avoid the singularity at $k_{t}=\Lambda_{Q C D}$. The freezing is usually unimportant, however, as the dominant contribution to the integral for a $120 \mathrm{GeV}$ Higgs boson at the LHC comes from the region around $Q_{t}=2 \mathrm{GeV}$. A smaller value of $\Lambda_{Q C D}$ results in a larger value of $\alpha_{s}$ at the same scale and hence enhances the probability for gluon emission and increases the Sudakov suppression.

For the un-integrated gluon densities (equation 2.37) LHAPDF [51] is used to provide the integrated gluon densities. The $R_{g}$ factor that accounts for the skewed effect is parameterised as $[28,50]$

$$
R_{g}=\left(1+\frac{\partial \ln \left(g\left(x, Q_{t}^{2}\right)\right)}{\partial x}\left(0.82+0.56 \frac{\partial \ln \left(g\left(x, Q_{t}^{2}\right)\right)}{\partial x}\right)\right) .
$$

Other than at small values of $Q_{t}$ or $x, R_{g}$ can be approximated by a constant value of 1.4 at Tevatron energies and 1.2 at LHC energies (figure 4.4).

The un-integrated gluon densities are approximated by $[50,52]$

$$
f_{g}=R_{g} \sqrt{T\left(Q_{t}, \mu\right)}\left(\frac{\partial x g\left(x, Q_{t}^{2}\right)}{\partial \ln \left(Q_{t}^{2}\right)}-\frac{N_{c} \alpha_{s}\left(Q_{t}\right)}{2 \pi} \ln \left(\frac{Q_{t}}{3\left(\mu+Q_{t}\right)}\right) x g\left(x, Q_{t}^{2}\right)\right)
$$

with the differentiation with respect to $Q_{t}$ being carried out numerically. The PDF has a lower limit to the range of $Q_{t}$ values for which it is valid and below that point is frozen, leading to the term in $\partial x g\left(x, Q_{t}^{2}\right) / \partial \ln \left(Q_{t}^{2}\right)$ in equation 4.5 


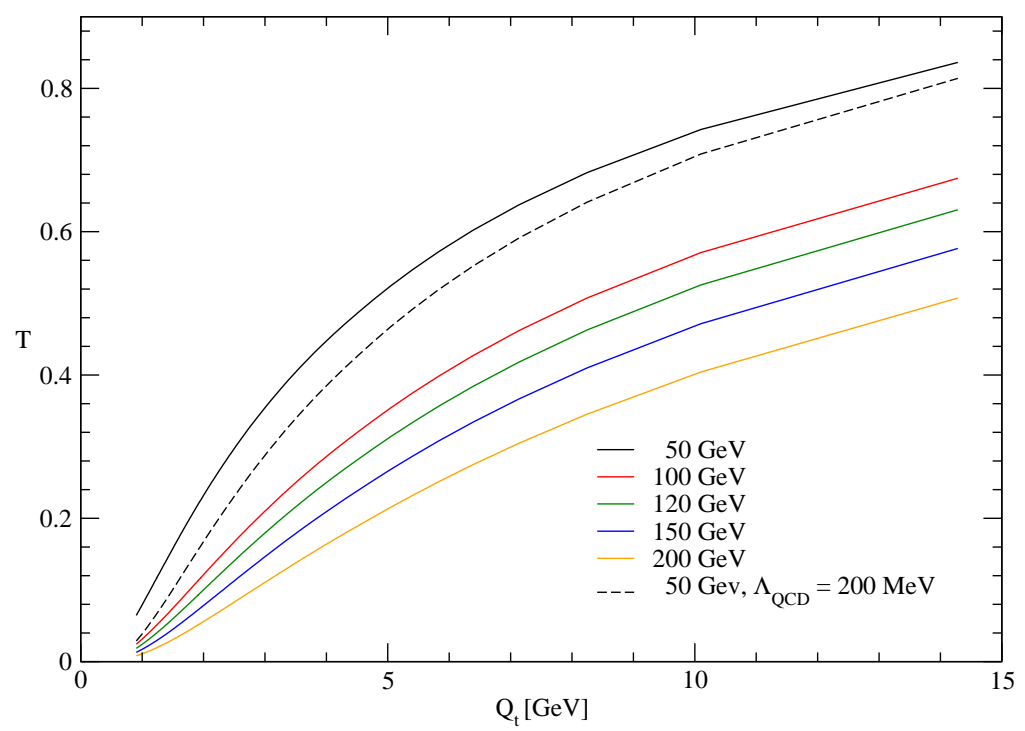

Figure 4.3: The Sudakov suppression, $T\left(Q_{t}, \mu\right)$, as implemented in ExHuME. $N_{f}$ (equation 4.3) was taken as 5 and, apart from the curve labelled $\Lambda_{Q C D}=200 \mathrm{MeV}, \Lambda_{Q C D}$ was chosen at $80 \mathrm{MeV}$ so that $\alpha_{s}\left(M_{z}\right) \simeq 0.118$. Curves are shown for central masses of 50,100, 120, 150 and $200 \mathrm{GeV}$, with the higher masses having a greater Sudakov suppression due to the larger phase space for emission of radiation that is available between $Q_{t}$ and $\mu$. The curve with $\Lambda_{Q C D}=200 \mathrm{MeV}$ shows a slightly greater suppression (i.e. smaller $T$ ) because the larger value of $\alpha_{s}$ results in a greater probability of radiation from the gluon lines.

vanishing. This produces the sharp peak seen in figure 4.5 as the PDF is abruptly cut off. LHAPDF has the facility to extrapolate the PDF below the lower $Q_{t}$ limit, which produces a smooth peak, but the extrapolation cannot be relied upon to give sensible values for the PDF. The lower limit of the integration in equation 2.41 must be cut off in any case to avoid the Landau pole in $\alpha_{s}$ at $Q_{t}=\Lambda_{Q C D}$, so ExHuME does not use the option to extrapolate the PDF. Cutting the integral off does not usually affect the result a great deal, as can be seen by comparing to the results contained in [27], where the author carrys out an extrapolation. The results are not strongly dependent on a cut on $Q_{t}$ that is below $1 \mathrm{GeV}$ because the most significant region of the integrand lies between 1 and $2 \mathrm{GeV}$. Above $Q_{t}=2 \mathrm{GeV}$ a change of variables is made in the integration parameter to $1 / Q_{t}^{2}$ so that $Q_{t}^{2}$ can be taken to a large upper limit yet the integration parameter remains small. The differential luminosity taken from ExHuME is shown in figures 4.6 


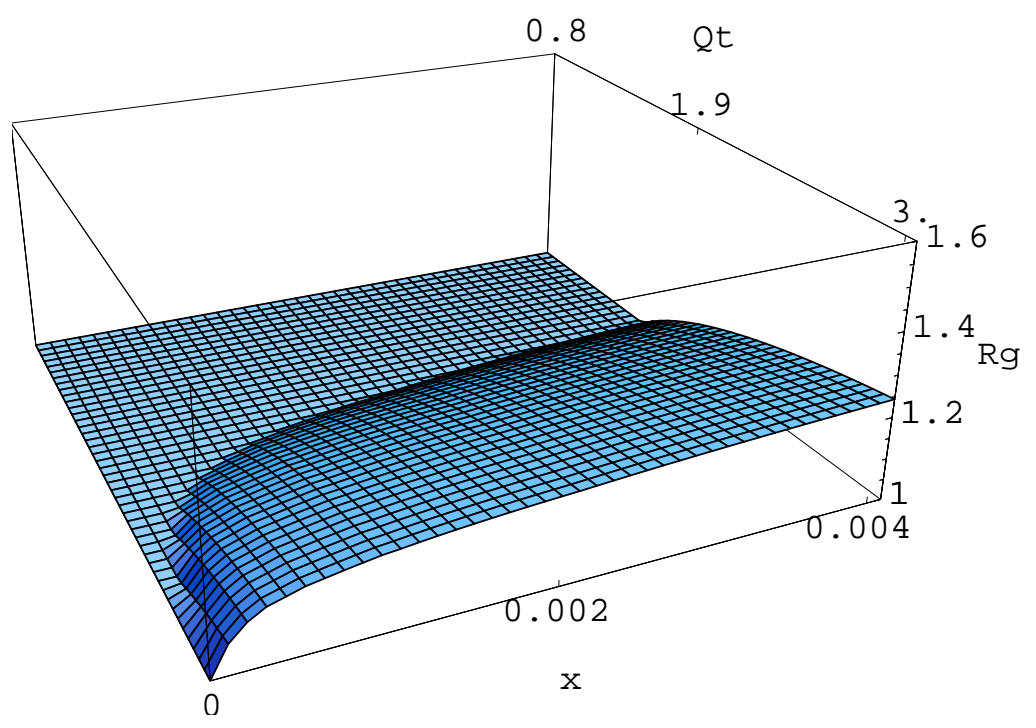

Figure 4.4: $R_{g}$ at the LHC.

and 4.7. The dimensionless quantity $M^{2} \partial^{2} L / \partial M^{2} \partial y$ shown in figures 4.6 and 4.7 has been integrated over $t_{1,2}$ and is related to equation 2.41 by

$$
\begin{aligned}
M^{2} \frac{\partial^{2} L}{\partial M^{2} \partial y} & =\int d t_{1} d t_{2} \exp \left(b\left(t_{1}+t_{2}\right)\right) M^{2} \frac{\partial^{2} \mathcal{L}}{\partial M^{2} \partial y} \\
& =\frac{1}{b^{2}} M^{2} \frac{\partial^{2} \mathcal{L}}{\partial M^{2} \partial y}
\end{aligned}
$$




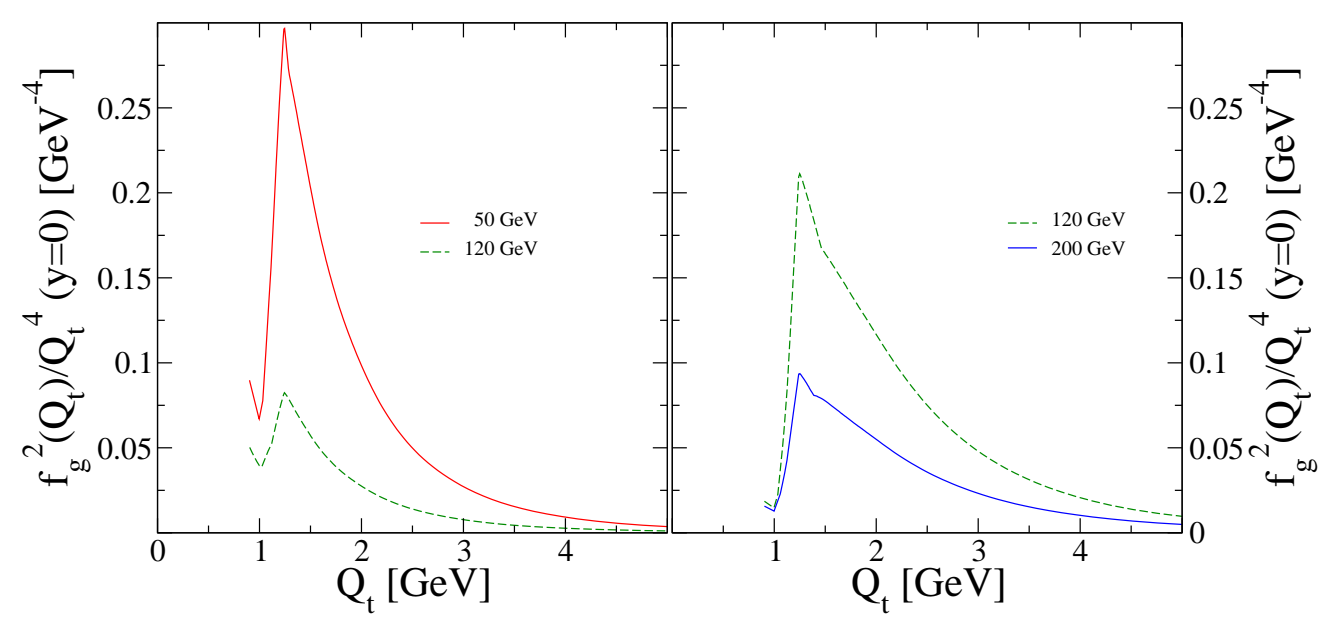

Figure 4.5: The function $f_{g}^{2}\left(Q_{t}\right) / Q_{t}^{4}$ evaluated at zero rapidity $\left(x_{1}=x_{2}\right)$ as implemented in ExHuME for Tevatron (left) and LHC (right) energies, both using the MRST 2002 PDF. The default ExHuME setup parameterises $R_{g}$ as in figure 4.4 and freezes the PDF below its lower $Q_{t}$, which results in a kink in the curve.
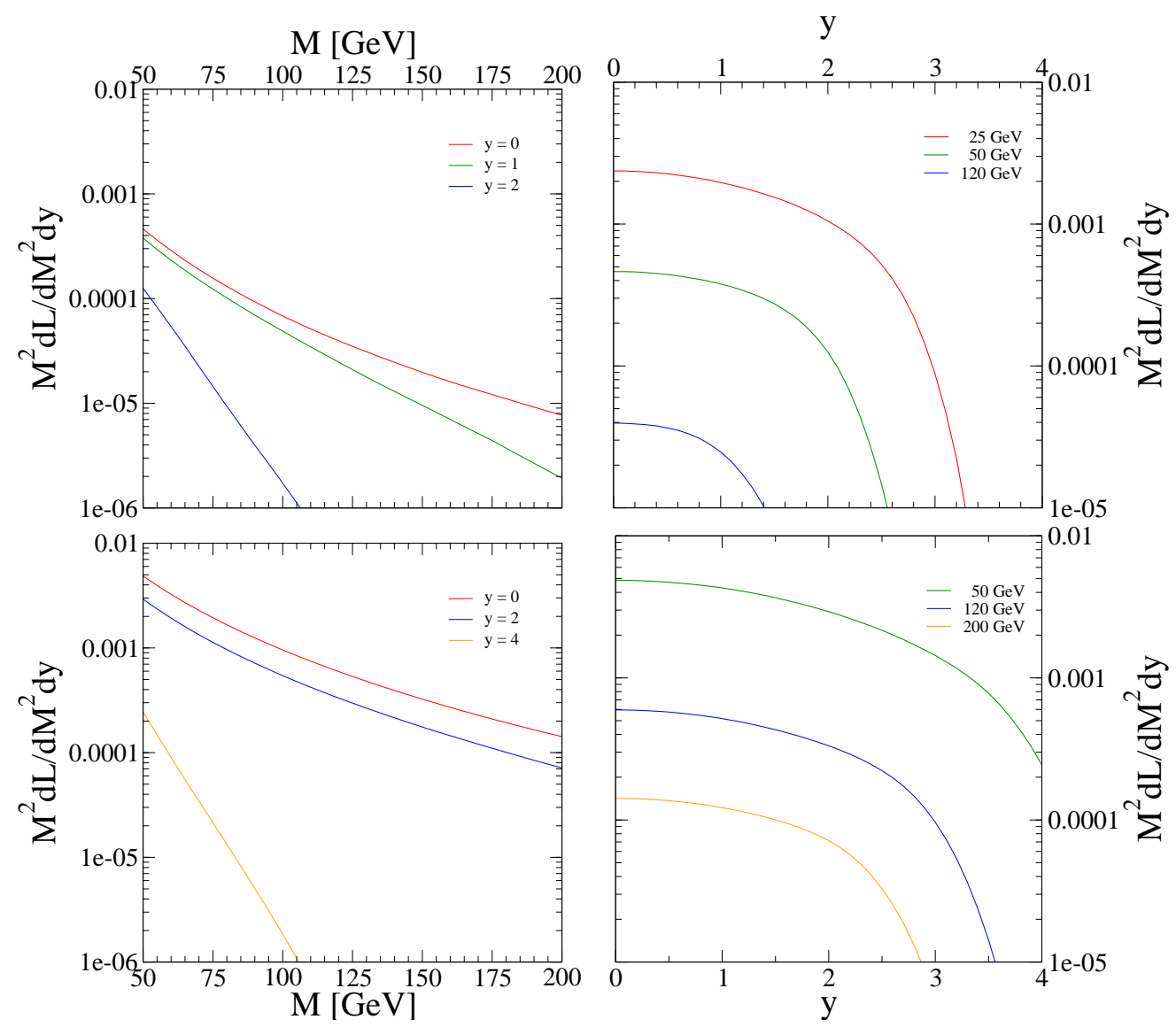

Figure 4.6: The mass (left column) and rapidity (right column) dependence of the differential luminosity function from ExHuME at the Tevatron (top row) and LHC (bottom row). The differential luminosity is given in equation 2.41 

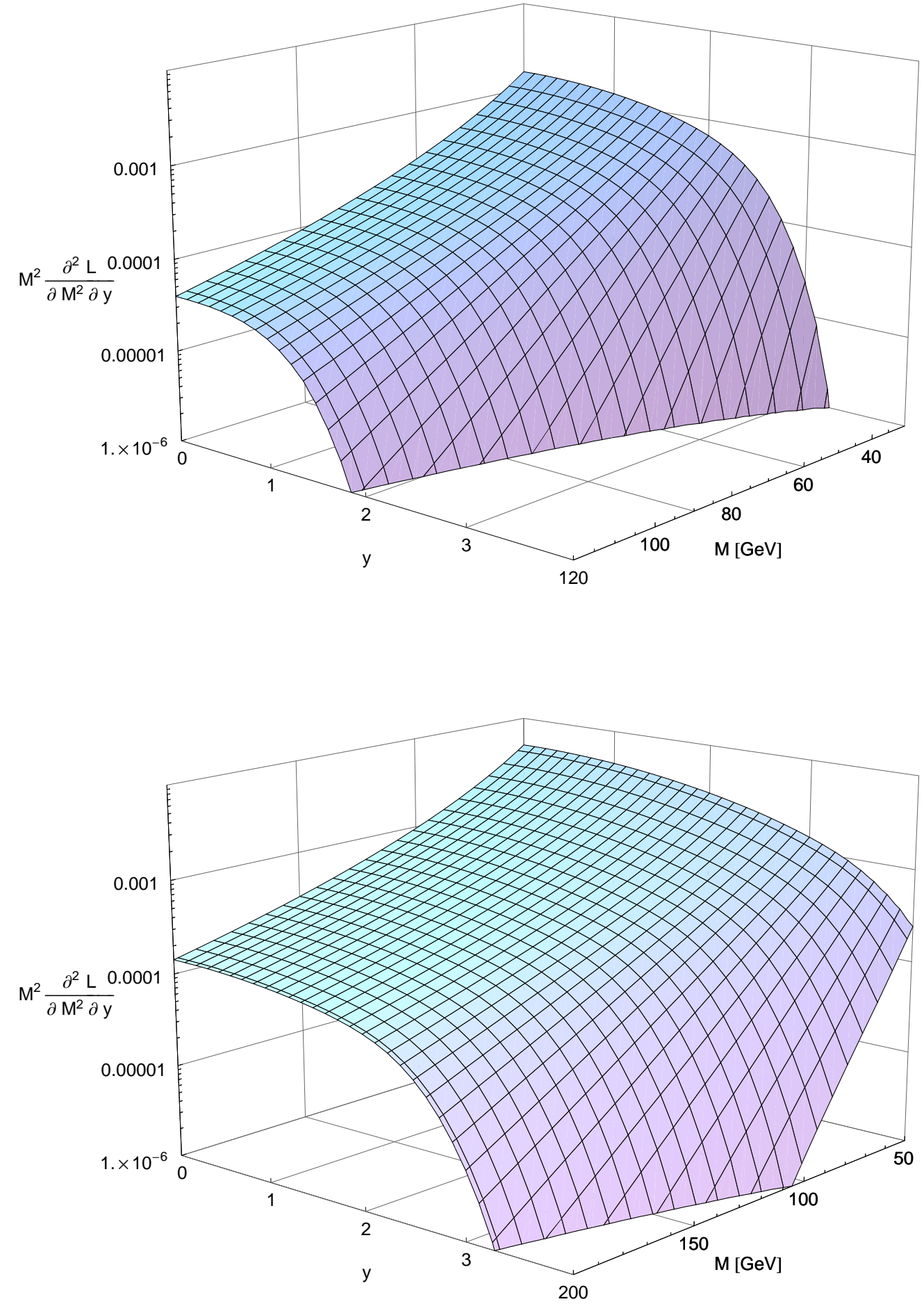

Figure 4.7: The mass and rapidity dependence of the differential luminosity taken from ExHuME for the Tevatron (top) and LHC (bottom). A soft survival factor of 0.045 was used at the Tevatron and 0.03 at the LHC. 


\subsection{Soft Survival Factor}

The current version of ExHuME uses a constant soft survival factor $\mathcal{S}^{2}$ (section 2.4) with default values of 0.03 for the LHC and 0.045 for the Tevatron. It is hoped that a future release will implement a soft survival factor with dependence on both the angle between the outgoing protons, $\phi_{p p}$, and the momentum transfers $t_{1,2}$, as is the case in the model in [3]. Adding a non-constant $\mathcal{S}^{2}$ will both harm the efficiency with which events are generated (section 4.5) due to the nontrivial dependence on $t_{1,2}$ and $\phi_{1,2}$ and will require further numerical integration for each calculation of the differential luminosity. It is therefore desirable that improvements to the speed and efficiency of ExHuME be made before the $t$ and $\phi$ dependence of $\mathcal{S}^{2}$ is added. Note that the soft survival factor does not significantly affect the kinematic distributions of the central system and that in any case, the model in [3] has an approximately flat $t_{1,2}$ dependence for $-t_{1,2}<(200 \mathrm{MeV})^{2}$.

\subsubsection{Sub Processes}

The original three subprocesses available in ExHuME were Standard Model Higgs boson production; direct production of a pair of (massive) quarks and direct production of a pair of gluons. A recent study added gluino pair production [53] and future work will include the $2 \rightarrow 3$ partonic level processes of $g g \rightarrow q \bar{q} g$, $g g \rightarrow q \bar{q} W$, exclusive production of a pair of photons and $\chi_{C}$ production.

The cross section for $g g \rightarrow H$ used for Higgs boson production is

$$
\hat{\sigma}(\hat{s}, y)=\frac{\alpha_{s}^{2}}{64 \pi} \frac{g^{2}}{M_{W}^{2}} \lambda(\hat{s})\left(\sum_{q} F_{s f}\left(\hat{s} /\left(4 m_{q}^{2}\right)\right)\right)^{2}
$$

where $g$ is the electroweak coupling, $M_{W}$ is the mass of the W boson, $m_{q}$ is the mass of the quark in the loop and the auxiliary function $F_{s f}$ is given in equation 3.11. The total width, $\Gamma_{t o t}$, and branching fractions of the Higgs boson are calculated using the fortran program Hdecay [54], which is linked into ExHuME. The Higgs boson line-shape function, $\lambda(\hat{s})$, is implemented as in [55]. A next to leading order QCD correction factor, $K$ is also used: 


$$
K=1+\frac{\alpha_{s}}{\pi}\left(\pi^{2}+\frac{11}{2}\right) \simeq 1.5 .
$$

The differential cross sections for $q \bar{q}$ and $g g$ production [1,2], when averaged over the gluon colours and polarisations (equation 2.36) and expressed in terms of the angle $\theta$ between the outgoing partons and the beam direction are:

$$
\begin{aligned}
\frac{\partial \hat{\sigma}_{q \bar{q}}}{\partial \cos (\theta)} & =\frac{4}{3} \frac{\pi \alpha_{s}^{2}(\hat{s})}{\hat{s} \sin ^{4}(\theta)} \frac{m_{q}^{2}}{\hat{s}}\left(1-4 \frac{m_{q}^{2}}{\hat{s}}\right)^{3 / 2}, \\
\frac{\partial \hat{\sigma}_{g g}}{\partial \cos (\theta)} & =18 \frac{\pi \alpha_{s}^{2}(\hat{s})}{\hat{s} \sin ^{4}(\theta)}
\end{aligned}
$$

It is more convenient to express equation 4.9 in terms of the angle $\theta$ in order to produce a weighted distribution in $\theta$ (section 4.5).

\subsection{Event Generation}

The efficiency of event generation is $n / N$, that is, $n$ events are generated for every $N$ attempts to generate an event, each of which requires the differential cross section to be calculated. Ideally, the efficiency would be made as close to one as possible in order that the differential cross section be computed as few times as possible. If the gluon fusion sub-process is resonant production then it would be very inefficient to simply pick the central mass $\sqrt{\hat{s}}$ from a set of uniformly distributed random numbers. For instance, a Standard Model Higgs boson with a mass of $120 \mathrm{GeV}$ has a width of $0.0036 \mathrm{GeV}$, so if the mass is allowed to roam freely over a range even as small as a $\mathrm{GeV}$ it will very rarely fall under the resonant peak.

To counter this, the random distributions of the kinematic variables can be modified so that, for instance, $\sqrt{\hat{s}}$ is most likely to be picked at the resonant mass. Ideally the distribution of the random numbers for $\sqrt{\hat{s}}$ would exactly match the final output distribution. This distribution can be achieved by calculating the following function: 


$$
R(\sqrt{\hat{s}})=\frac{\int_{\hat{s}_{0}}^{\hat{s}} \frac{\partial \sigma}{\partial \hat{s}^{\prime}} d \hat{s}^{\prime}}{\int_{\hat{s}_{0}}^{\hat{s}_{1}} \frac{\partial \sigma}{\partial \hat{s}^{\prime}} d \hat{s}^{\prime}}
$$

where $\hat{s}$ is in the range $\left\{\hat{s}_{0}, \hat{s}_{1}\right\}$. R will always lie between 0 and 1 and the weighted random distribution for $\sqrt{\hat{s}}$ is given by

$$
\sqrt{\hat{s}}=R^{-1}(r)
$$

where $R^{-1}$ is the inverse function to $R$ and $r$ is a random number distributed uniformly in $\{0,1\}$. This is shown in figure 4.8 where a set of uniform random numbers on the vertical axis is mapped onto a Breit-Wigner distribution on the horizontal axis using the integral of the Breit-Wigner shape.

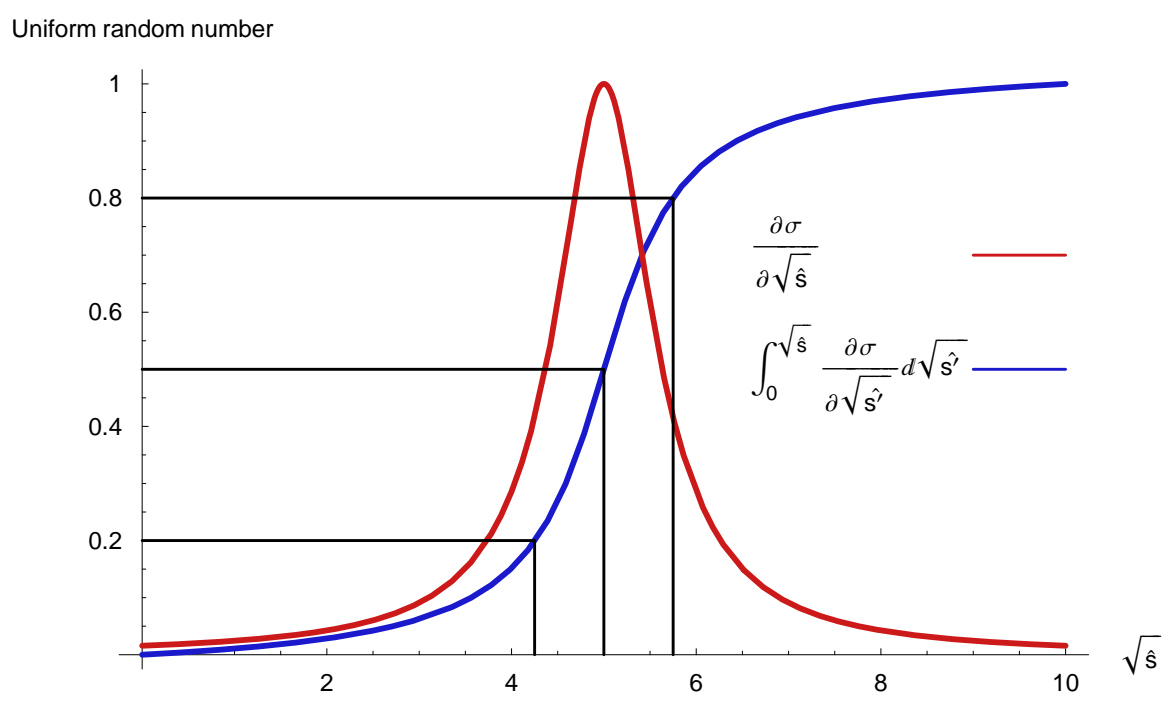

Figure 4.8: Producing a Breit-Wigner distribution for $\hat{s}$ from a set of random numbers distributed uniformly in $\{0,1\}$.

ExHuME also uses this method to create a distribution for $t_{1}$ and $t_{2}$. Ignoring the soft survival factor, the $t$ dependence of the differential cross section is $e^{b\left(t_{1}+t_{2}\right)}$. Using equation 4.10 for the $t$ dependence gives

$$
R(t)=\frac{e^{b t}-e^{b t_{\min }}}{e^{b t_{\max }}-e^{b t_{\min }}}
$$

so that the weighted random number distribution used for $t$ is 


$$
t(r)=\frac{1}{b} \ln \left(r\left(e^{b t_{\max }}-e^{b t_{\min }}\right)+e^{b t_{\min }}\right)
$$

where $r$ is a random number distributed uniformly in $\{0,1\}$. Using this distribution for $t_{1}$ and $t_{2}$ means that there is no need to reject events. Effectively the rectangle around the peak in figure 4.2 has been replaced by the shape of the differential cross section and the efficiency is one.

It is not always possible to evaluate $R$ analytically and this is the case with the $\sqrt{\hat{s}}$ dependence of the differential cross section. However, it is always possible to evaluate $R$ using numerical integration. ExHuME samples the differential cross section and creates a look-up table of $\sqrt{\hat{s}}$ indexed by $R$. The differential cross section may be sharply peaked, so the look-up table is created in three iterations. The first iteration calculates $R$ at even intervals in $\sqrt{\hat{s}}$, while the second and third iterations use the previous iteration of the look-up table and pick further points evenly spaced in $R$ so that the map is most detailed in regions where the differential cross section is most sharply peaked. The beauty of this method is that, unless the differential cross section is exceptionally pathological, it is valid for any process. The same technique is used to sample the integrand in equation 2.41 for the numerical integration over $Q_{t}^{2}$.

In constructing the map, the differential cross section is sampled over the region of interest and the results are used to replace the rectangle of figure 4.2 with an approximation to the shape of the differential cross section (figure 4.10). It is possible that the approximate fit might clip the top off the true shape of the function; if ExHuME encounters such a point it will add a new point to the shape of the fit and re-calculate the volume. 


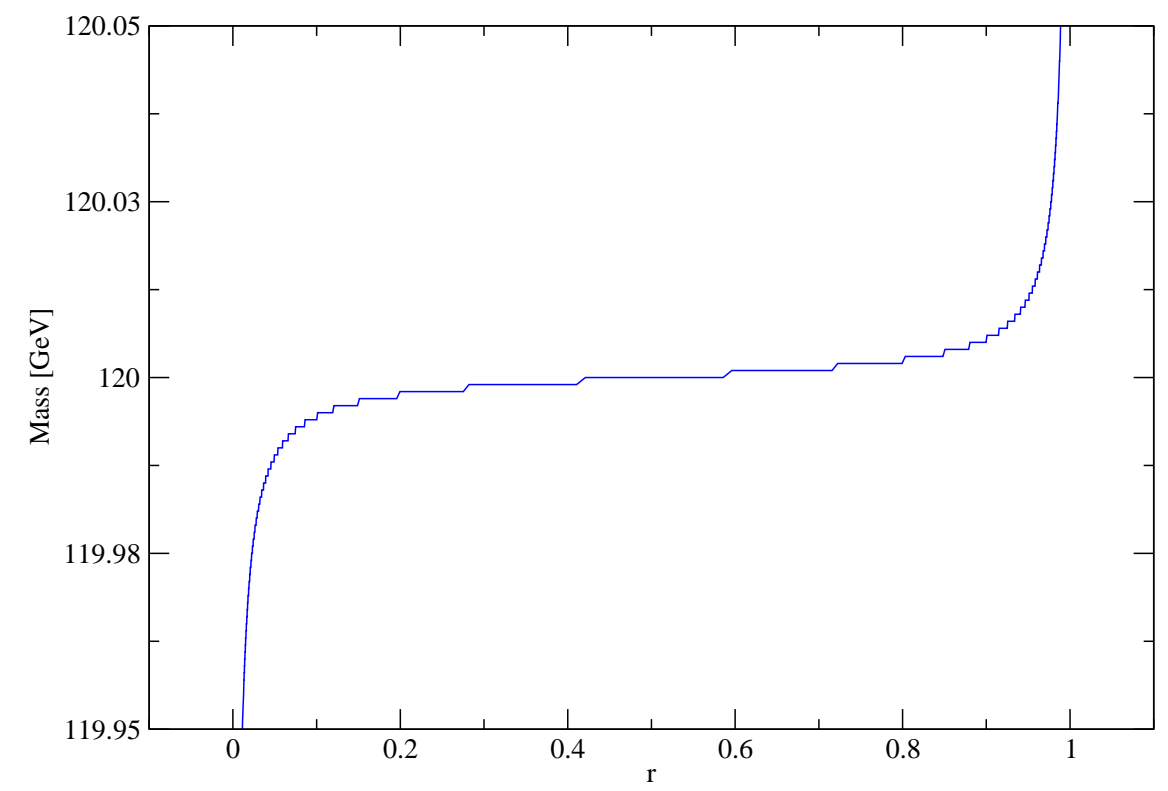

Figure 4.9: Map taken from ExHuME mapping a uniformly distributed random number $r$ to a weighted distribution of masses that is strongly peaked about a resonant mass of $120 \mathrm{GeV}$.

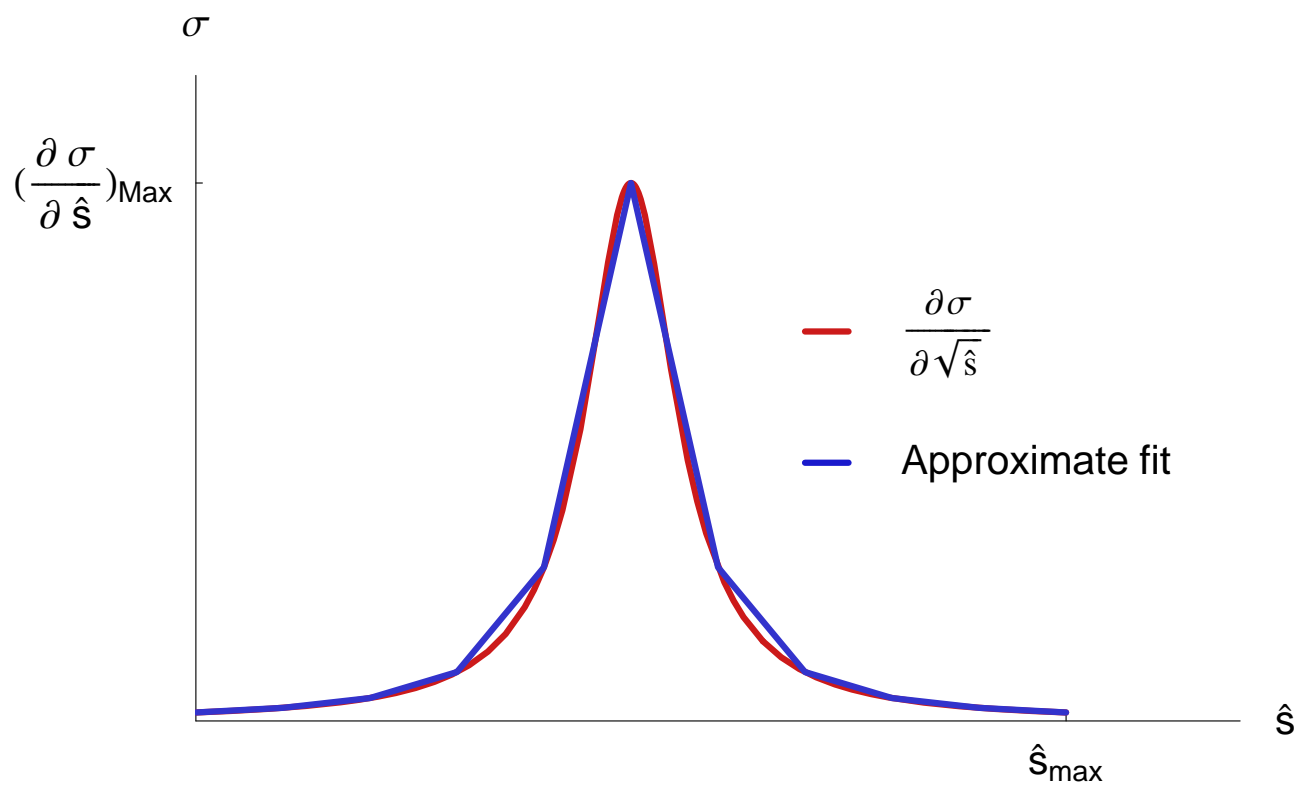

Figure 4.10: A resonant differential cross section and an approximate fit to it. The fit clips the top of the true function.

The efficiency of event generation for production of a resonance is usually 
around $40 \%$ and is almost independent of the allowed mass range that is chosen. For example, even if the mass range is set between 50 and $1000 \mathrm{GeV}$ for 120 GeV Higgs boson production, the efficiency is around 38\%, compared to $39 \%$ if the allowed mass range is set to be $120 \pm 1 \mathrm{GeV}$. The reason that the efficiency is around $40 \%$ and not much higher is because the central rapidity is chosen from a uniform distribution. This has to be done because the rapidity of an event is not independent of its central mass; a higher central mass permits a larger rapidity range. Figure 4.11 shows the cross section result that would be obtained for each event were the simulation to be stopped at that point, as well as the standard deviation of that result. The speed with which the cross section converges towards the final result is sufficient to engender confidence in the result, with fluctuations of less then $1 \%$ by the $10,000^{\text {th }}$ event and reasonable convergence with 1000-2000 events. 

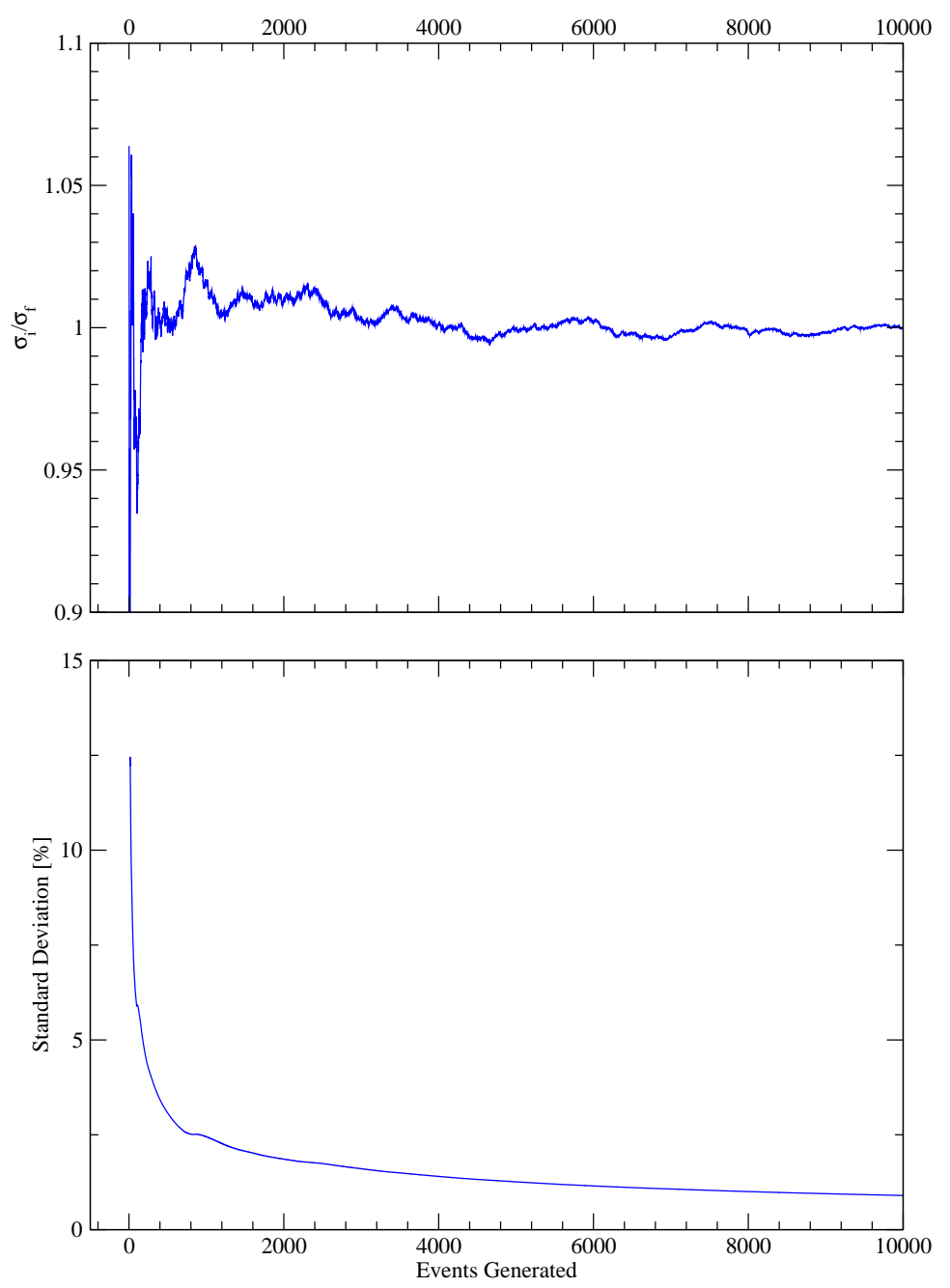

Figure 4.11: Convergence of ExHuME over 10,000 events for Higgs boson production $\left(M_{H}=120 \mathrm{GeV}\right)$. The top plot shows the evolution of the cross section measurement, $\sigma_{i}$, towards the final cross section result $\sigma_{f}$. The lower plot shows the standard deviation of the result versus number of events generated.

\subsubsection{Parton Shower and Hadronisation}

ExHuME produces a partonic system such as a Higgs boson or a pair of quarks. To evolve the parton level system to a stable final state system that reaches the detector, a parton shower and a hadronisation routine are needed. ExHuME passes this duty to the Pythia Monte Carlo generator [56]. Happily, there is no initial state radiation to take account of in central exclusive production, so all 
that is necessary is for ExHuME to pass the four vectors of the outgoing partons to Pythia make the necessary colour connections and execute Pythia's parton showering and hadronisation routines.

A parton shower routine uses the Sudakov form factor that gives the probability that a parton does not radiate between two given scales. The parton shower has to be cut off at some lower scale limit to avoid entering the non-perturbative regime. The Sudakov form factor is used, then, as the generating function for a Monte Carlo distribution of the scales at which the partons do split, with the condition that the emitted partons are well ordered in virtuality; higher virtuality partons are radiated first. The non-perturbative hadronisation model is used once the parton shower has been run down to the lower cut off scale. Pythia uses the colour string model in which strings under tension are stretched between all colour connected objects. Once the energy stored in the string is sufficient to create a new pair of coloured objects the string "snaps." For further details of the Pythia parton shower algorithm and hadronisation model see the Pythia manual [56].

\subsection{Results from ExHuME}

\subsubsection{Higgs Boson Production}

The cross section for central exclusive production of a $115 \mathrm{GeV}$ Standard Model Higgs boson at the Tevatron is predicted to be around $0.2 \mathrm{fb}$. Two such Higgs Bosons would therefore be available in the combined integrated luminosity collected by CDF and DØ over the course of the Tevatron Run II. Results for Higgs boson production shall therefore be shown for the LHC only. The Higgs boson production cross section at Higgs boson masses between 100 and $200 \mathrm{GeV}$ is shown in figure 4.12. Note that if the Higgs boson has a mass of around 140 $\mathrm{GeV}$ or more then the $H \rightarrow W W$ channel may well prove the most favourable for studying the Higgs boson, especially given that it will be far easier to trigger on a $W$ jet rather than a $b$ jet at the LHC [57].

Figure 4.13 is shown only for the purpose of verifying that ExHuME has correctly reproduced the resonant peak. The rapidity distributions of the Higgs 


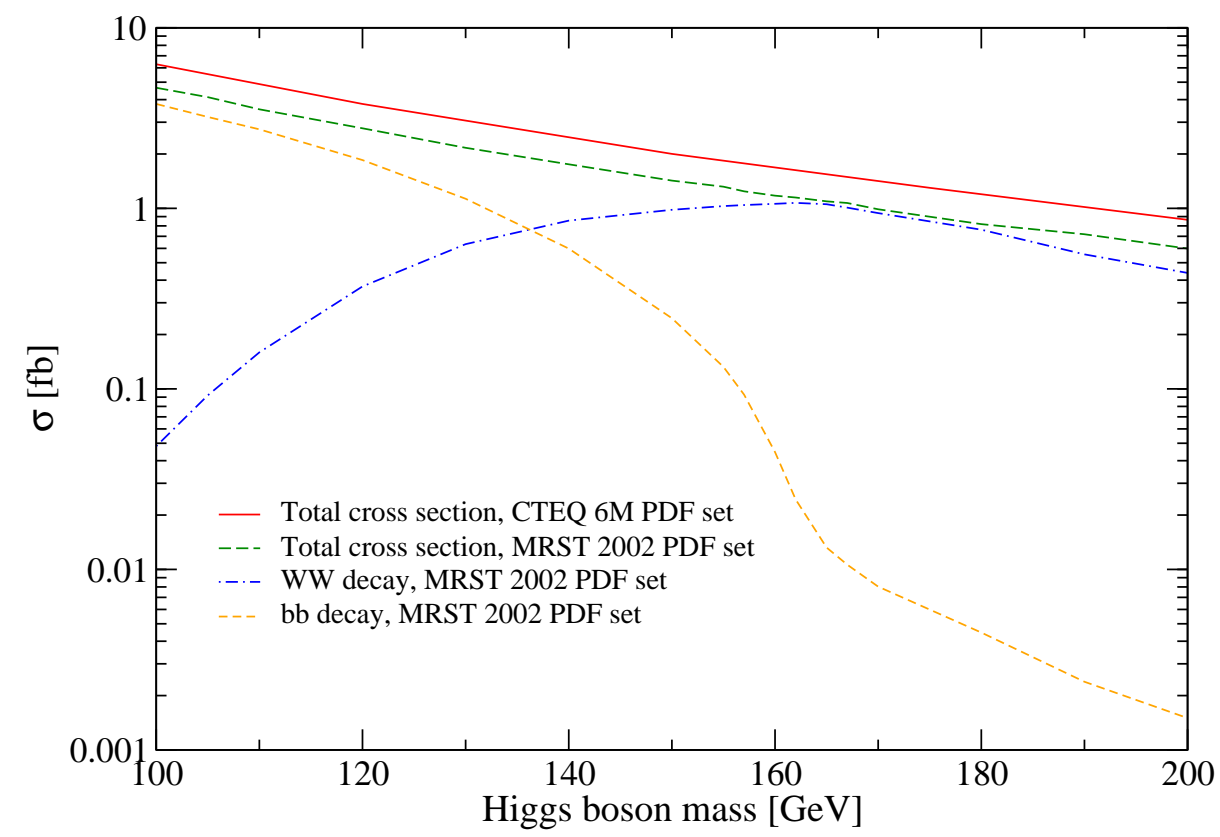

Figure 4.12: Standard model Higgs boson production cross section taken from ExHuME. The CTEQ6 PDF set gives a slightly higher cross section than the MRST 2002 set. At masses around $180 \mathrm{GeV}$ the $H \rightarrow Z Z$ decay channel starts to become significant, which is why the $W W$ channel falls away from the total production cross section at that point.

boson and its most forward decay product at a mass of $120 \mathrm{GeV}$ are shown in figure 4.14 and confirm that most of the cross section lies entirely within a rapidity range of \pm 4 . The rapidity of the most forward decay product from a 150 GeV Higgs boson in the $W W^{*}$ channel (all $W$ decay modes) is also given for comparison in the same figure and shows that the different decay mode makes little difference to the rapidity coverage of the event.

The centrally peaked rapidity distribution of the Higgs boson is caused by the gluon distributions favouring the lowest possible $x$; figure 4.15 shows the $x$ distributions for a $120 \mathrm{GeV}$ Higgs boson. Given that the product $x_{1} x_{2}=m_{H}^{2} / s$, there is an upper limit to the lower of the two $x$ values, $x_{\text {Min }}$ and a lower limit to the larger of the two $x$ values, $x_{\text {Max }}$. These limits correspond to the zero rapidity case $x_{\text {Min }}=x_{\text {Max }}=m_{H} / \sqrt{s} \simeq 0.009$ for a $120 \mathrm{GeV}$ Higgs boson at the LHC. Since $x_{\text {Max }}<1, x_{\text {Min }}$ is constrained to be between $m_{H}^{2} / s$ and $m_{H} / \sqrt{s}$ and the 
kinematically allowed range of $x_{M i n}$ is far smaller than that for $x_{M a x}$. The gain in gluon flux due to picking $x_{\min }$ below the limit is then more than offset by the loss in gluon flux caused by having to pick $x_{M a x}$ above the limit to maintain the same central mass. This correlation between the $x_{1,2}$, which favours a central distribution of the Higgs boson rapidity, has led to an increase in the predicted detector acceptances [58] for the proposed forward proton tagging system at the LHC over other models in which the Higgs boson rapidity distribution is flatter [59].

The $t$ distribution of the protons is shown in figure 4.16 and, as expected, returns the simple $t$ dependence of $e^{b t}$ used. Adding a more complicated $t$ dependence to the soft survival factor will create correlations in the transverse momenta of the outgoing protons and could change the proton tagger acceptances given in [58].

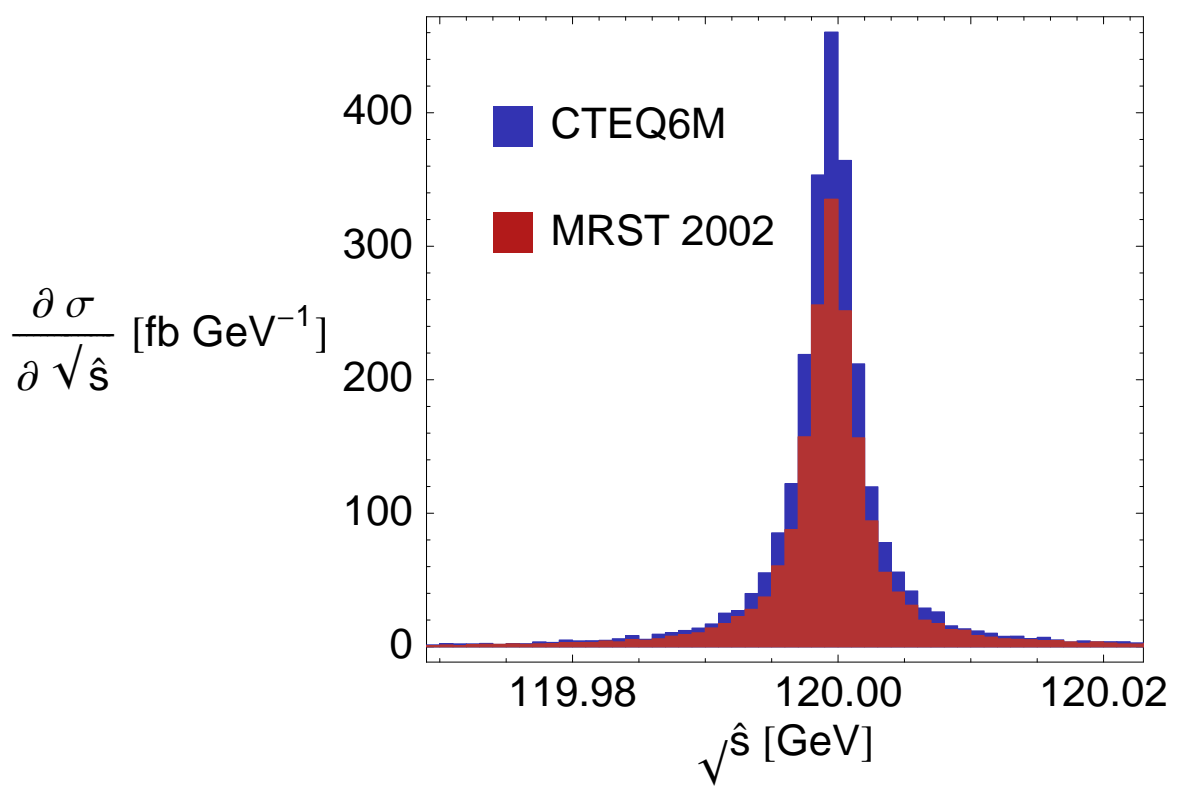

Figure 4.13: Mass of the centrally produced $120 \mathrm{GeV}$ Higgs boson.

A cone algorithm defines a jet to be all particles lying within a cone of a specified radius. The radius, $R_{c}$, of the cone at rapidity $\eta$ and azimuthal angle $\phi$ is defined as 


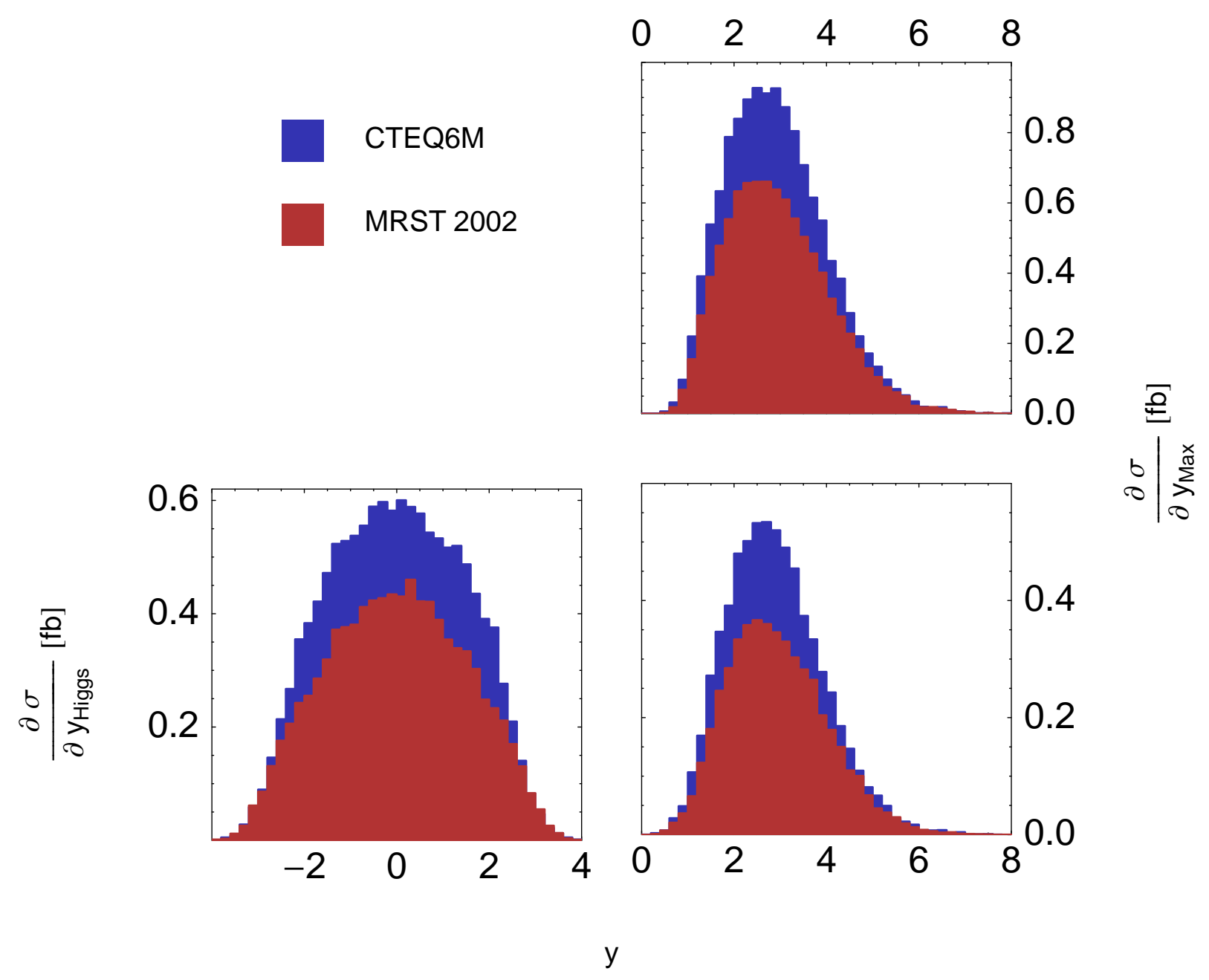

Figure 4.14: The rapidity of a $120 \mathrm{GeV}$ Higgs boson (bottom left) and the rapidity of the most forward particle (outgoing protons excepted) for a $120 \mathrm{GeV}$ Higgs boson in the $H \rightarrow b \bar{b}$ channel (top right) and $150 \mathrm{GeV}$ Higgs boson in the $H \rightarrow W W$ channel (bottom right).

$$
R_{c}^{2}=(\Delta \eta)^{2}+(\Delta \phi)^{2}
$$

where $\Delta \eta$ and $\Delta \phi$ are the extent of the cone in $\eta-\phi$. Since rapidity is additive under Lorentz boosts, the size $\Delta \eta$ of the cone is independent of the rapidity at which the cone lies. Cone algorithms locate the centre of the cone by iteratively merging or clustering neighbouring particle four-vectors.

An overlap parameter specifies how much of the energy inside one cone may be derived from particles already lying inside other jets. The pxcone [60] cone 

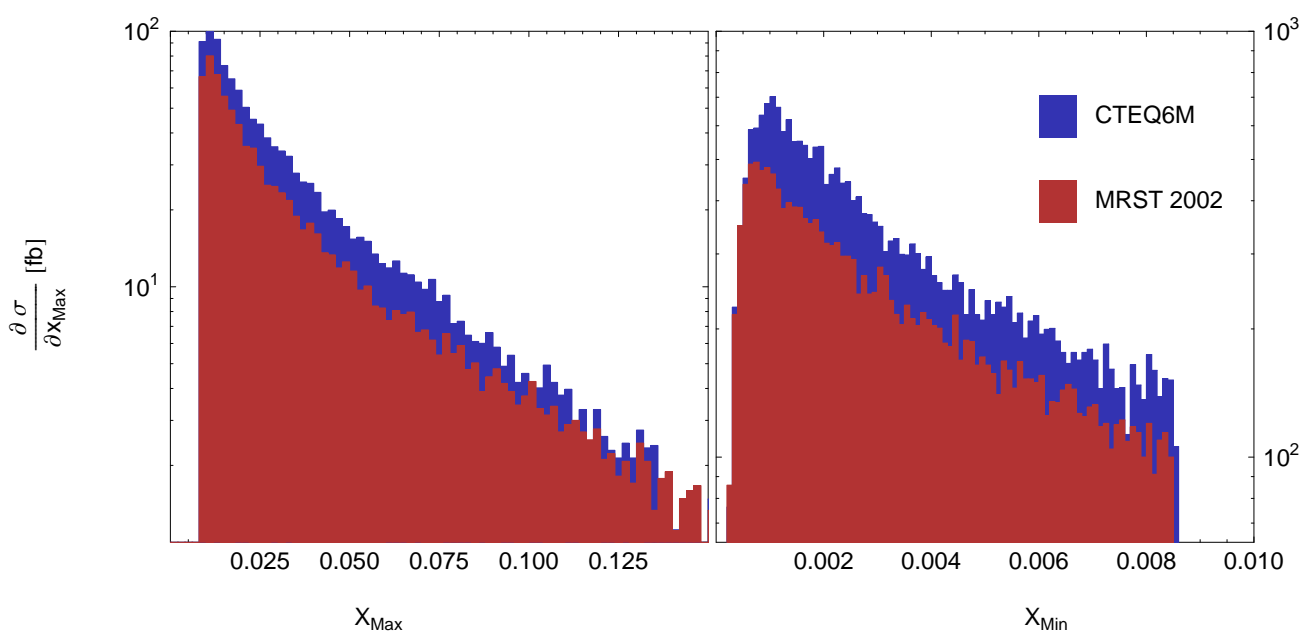

Figure 4.15: The $x$ distributions for a $120 \mathrm{GeV}$ Higgs boson.

algorithm with a cone radius of 0.7 and overlap parameter of 0.5 was run on the final state output from ExHuME and the $E_{T}$ distribution of the largest jet is shown in figure 4.17.

\subsubsection{Direct b Jet Production at the LHC}

A major background to exclusive Higgs boson production at $120 \mathrm{GeV}$ will be the direct production of a pair of $b$ flavoured jets. This is suppressed by a factor of $m_{b}^{2} / m_{H}^{2}$, so the higher order processes with additional gluon radiation, which are not suppressed, may be very important. However, $2 \rightarrow 3$ partonic processes [61] have not yet been implemented in ExHuME, although it is anticipated that they will be added in the near future. For this reason only the $2 \rightarrow 2$ background to Higgs boson production will be shown here.

The cross section for production of a $b \bar{b}$ pair at the LHC with an allowed central mass of between 119 and $121 \mathrm{GeV}$ (the proton taggers are expected to have a mass resolution of $1 \mathrm{GeV}$ ) in ExHuME is $2.65 \mathrm{fb}$ using the MRST 2002 PDF set [23]. Figure 4.17 shows that the $1 / E_{T}^{4}$ dependence of the $g g \rightarrow q \bar{q}$ cross section produces an $E_{T}$ distribution that is completely different from the signal of isotropic decay of the scalar Higgs boson, $H \rightarrow q \bar{q}$. A cut on the $E_{T}$ of the jets can therefore be made to increase the statistical significance, signal $/ \sqrt{(\text { signal }+ \text { background })}$. The significance versus the $E_{T}$ cut for an inte- 


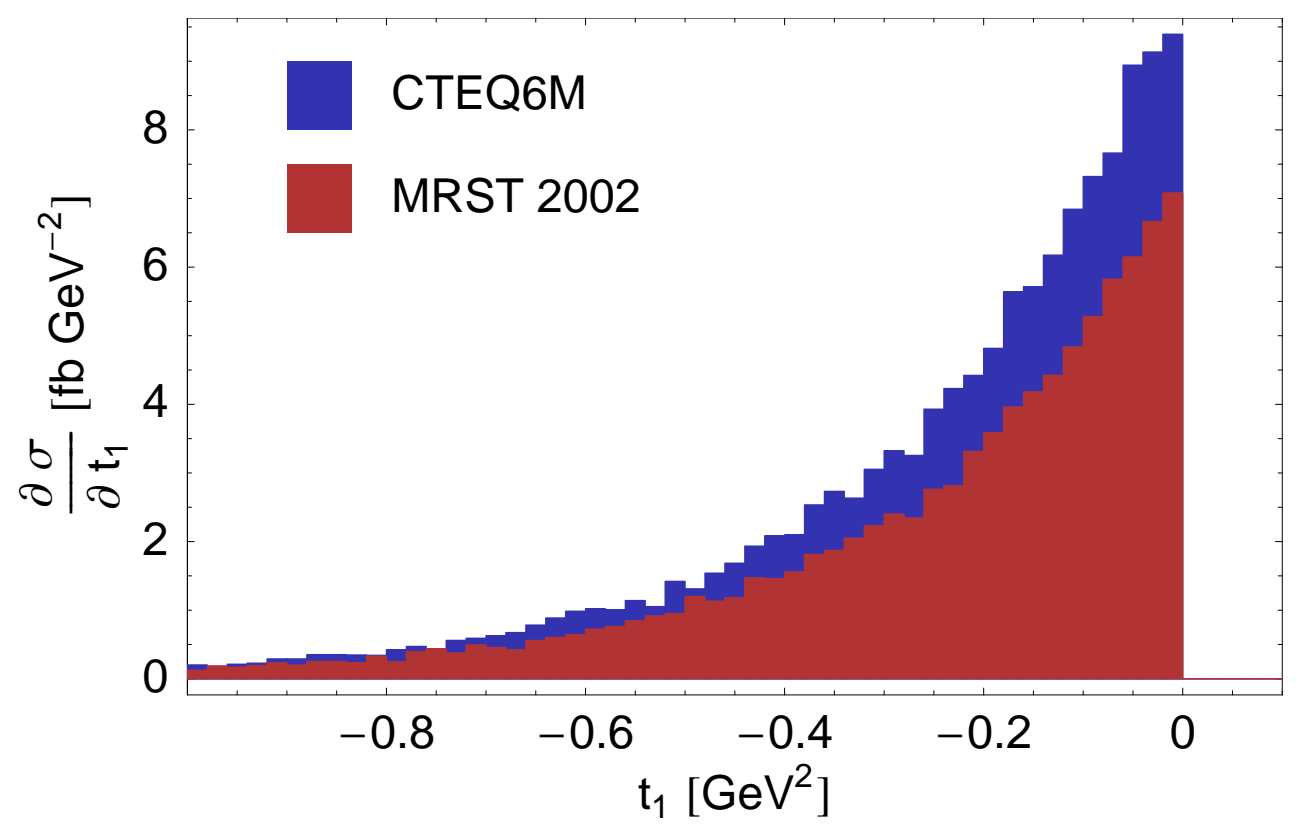

Figure 4.16: The $t$ distribution for a $120 \mathrm{GeV}$ Higgs boson.

grated luminosity of $1 \mathrm{fb}^{-1}$ is shown in figure 4.18 , which reveals that an $E_{T}$ cut should be made at approximately $32 \mathrm{GeV}$ and that at least $25 \mathrm{fb}^{-1}$ of total integrated luminosity will be required for a $5 \sigma$ discovery of the Higgs boson using central exclusive production. Further backgrounds such as inclusive double pomeron exchange and exclusive $b \bar{b} g$ production exist. This, together with the fact that the LHC experiments will not be able to trigger on and record every event and that any forward proton taggers will have a finite acceptance in $x_{1,2}$ and $t_{1,2}$, will mean that more than $25 \mathrm{fb}^{-1}$ will be needed.

The central detector will not be perfect at identifying $b$ flavoured jets, and a certain number of jets that arise from gluons will be miss-identified as $b$ jets. This will further increase the background to Standard Model Higgs production, depending on the $b$-tagging efficiency. However, the central exclusive production of a pair of gluons has the same $1 / E_{T}^{4}$ dependence as the production of a pair of quarks, so the $E_{T}$ cut should still be made at $32 \mathrm{GeV}$. The cross section given by ExHuME for the production of a pair of gluons with a total central mass of between 119 and $121 \mathrm{GeV}$ is $24 \mathrm{pb}$. 


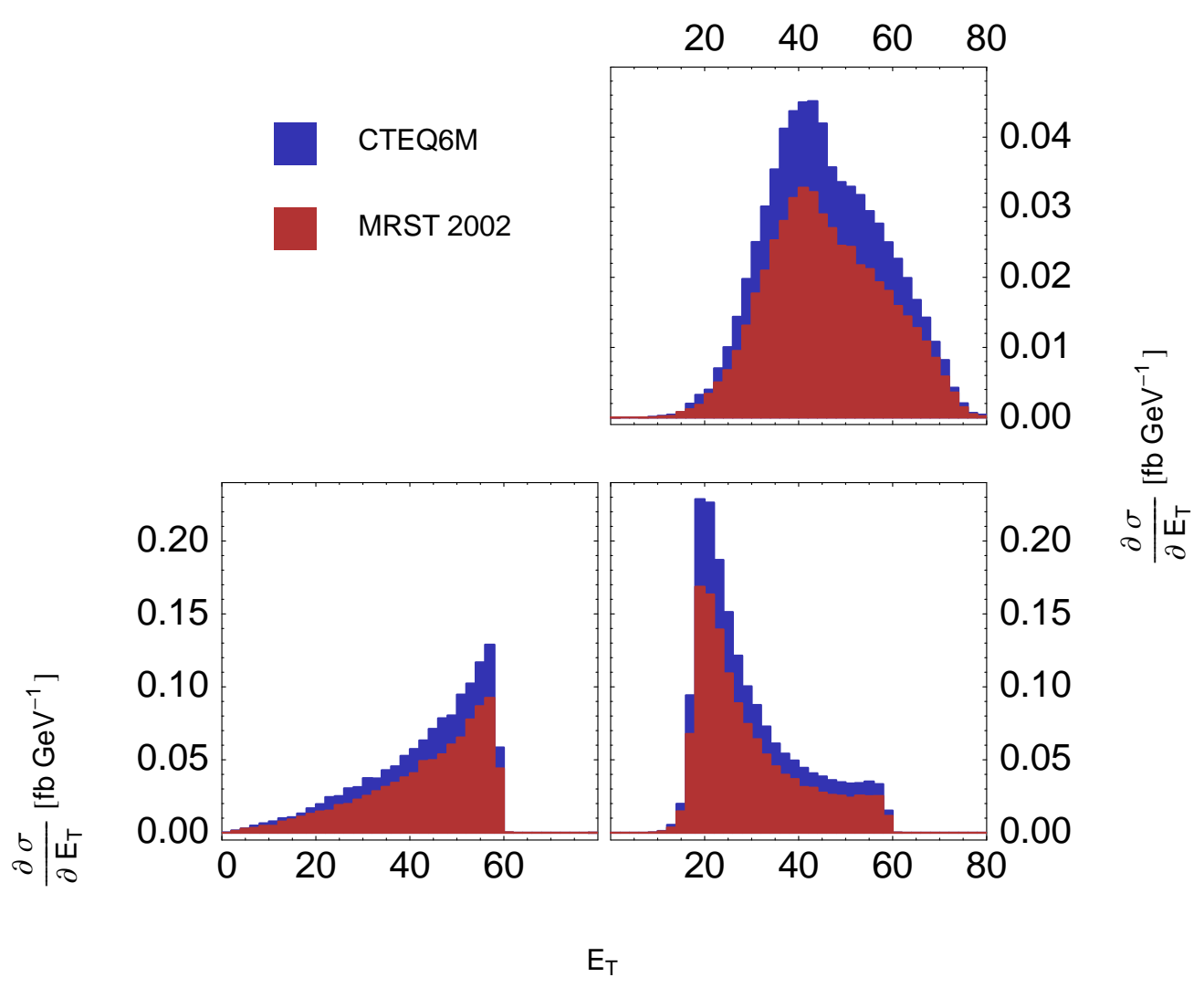

Figure 4.17: The $E_{T}$ of the largest $E_{T}$ jet for a $120 \mathrm{GeV}$ Higgs boson in the $H \rightarrow b \bar{b}$ channel (bottom left) and the background of direct $b \bar{b}$ pair production with a central mass between 119 and $121 \mathrm{GeV}$ (bottom right). Also shown is the largest jet $E_{T}$ from a $150 \mathrm{GeV}$ Higgs boson in the $H \rightarrow W W^{*}$ channel (top right).

\subsubsection{Di-Jet Production at the Tevatron}

Central exclusive production is not a feasible search channel for the Higgs boson at the Tevatron, but higher rate process can still be studied in order to validate the model. Such processes include exclusive $\chi_{C}$ production [62] and exclusive di-photon production [9], although the lower mass of those systems may call into question the leading order perturbative QCD calculation of central exclusive production. The highest rate process, however, should be hadronic di-jet production.

Half a million $q \bar{q}$ and $g g$ events were generated using ExHuME, with the type of parton being in proportion to its cross section. The events were allowed to have a central mass in the range $\{10,80\} \mathrm{GeV}$ with a minimum transverse 


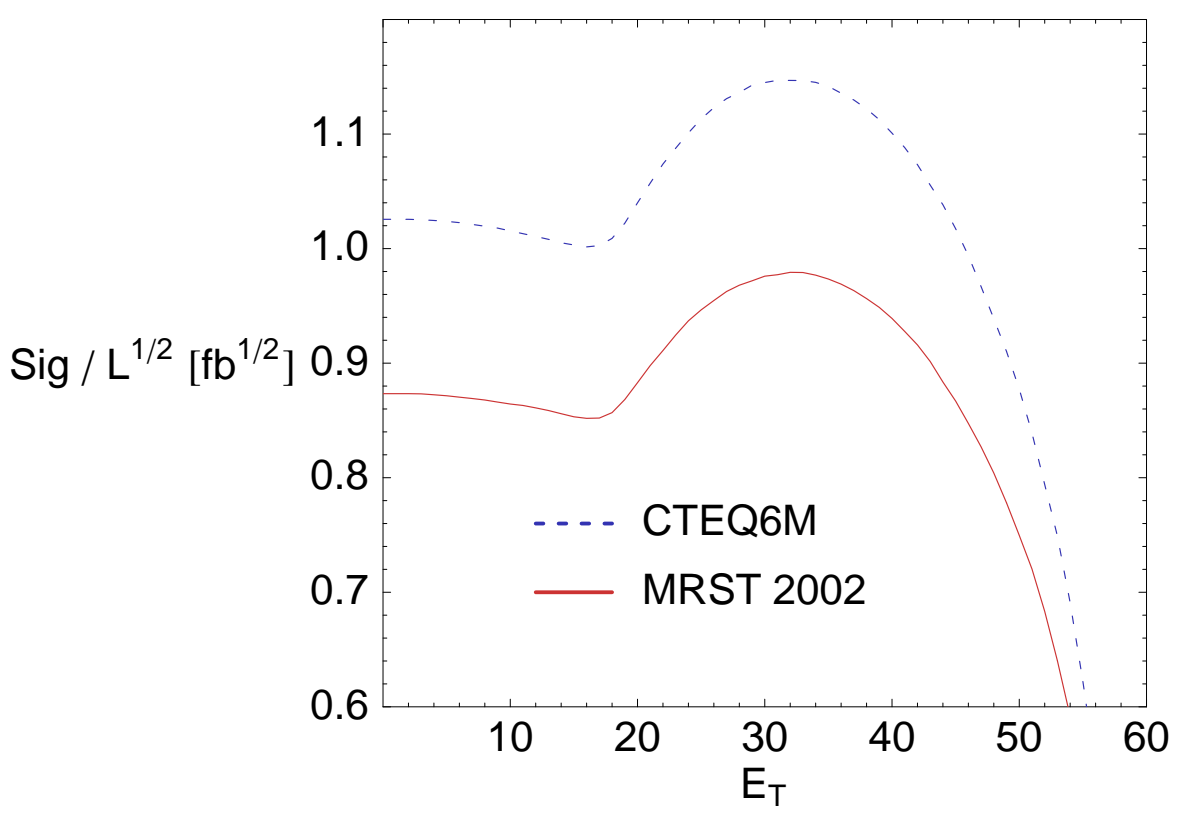

Figure 4.18: The statistical significance in $\sigma$ per $\mathrm{fb}^{-1 / 2}$ of integrated luminosity $1 / 2$ Vs. the lower cut on $E_{T}$ that is made.

momentum for the outgoing partons of $3 \mathrm{GeV}$. Gluon production dominated with the largest cross section of $2.8 \times 10^{6} \mathrm{pb}$ and, since the quark production cross section is proportional to the quark mass squared, $b \bar{b}$ production was the only other notable contribution with a cross section of $7.0 \times 10^{3} \mathrm{pb}$. The pxcone cone algorithm with a cone radius of 0.7 and an overlap parameter of 0.5 was run on the final state system in order to identify jets.

The central mass distribution from ExHuME is shown in figure 4.19 and reflects both the fall in the differential luminosity with $\sqrt{\hat{s}}$ and the $1 / \hat{s}^{2}$ dependence of the central $g g \rightarrow g g$ cross section. Figure 4.19 also demonstrates that, especially for low mass events, not all of the final state particles will be identified with a jet. If a cut is made on the largest jet $E_{T}$ of $7 \mathrm{GeV}$ then many events are lost and the distribution has a peak at around $\sqrt{\hat{s}}=20 \mathrm{GeV}$, even though, with a lower $\sqrt{\hat{s}}$ limit of $14 \mathrm{GeV}$ one might expect most events to contain one quark with an 
$E_{T}$ above $7 \mathrm{GeV}$ at the parton level. The $E_{T}$ cut does not affect the upper end of the $\sqrt{\hat{s}}$ distribution. The $E_{T}$ distributions of the first and second jet are shown in figure 4.20 and show that the $E_{T}$ of the second jet closely matches the first.

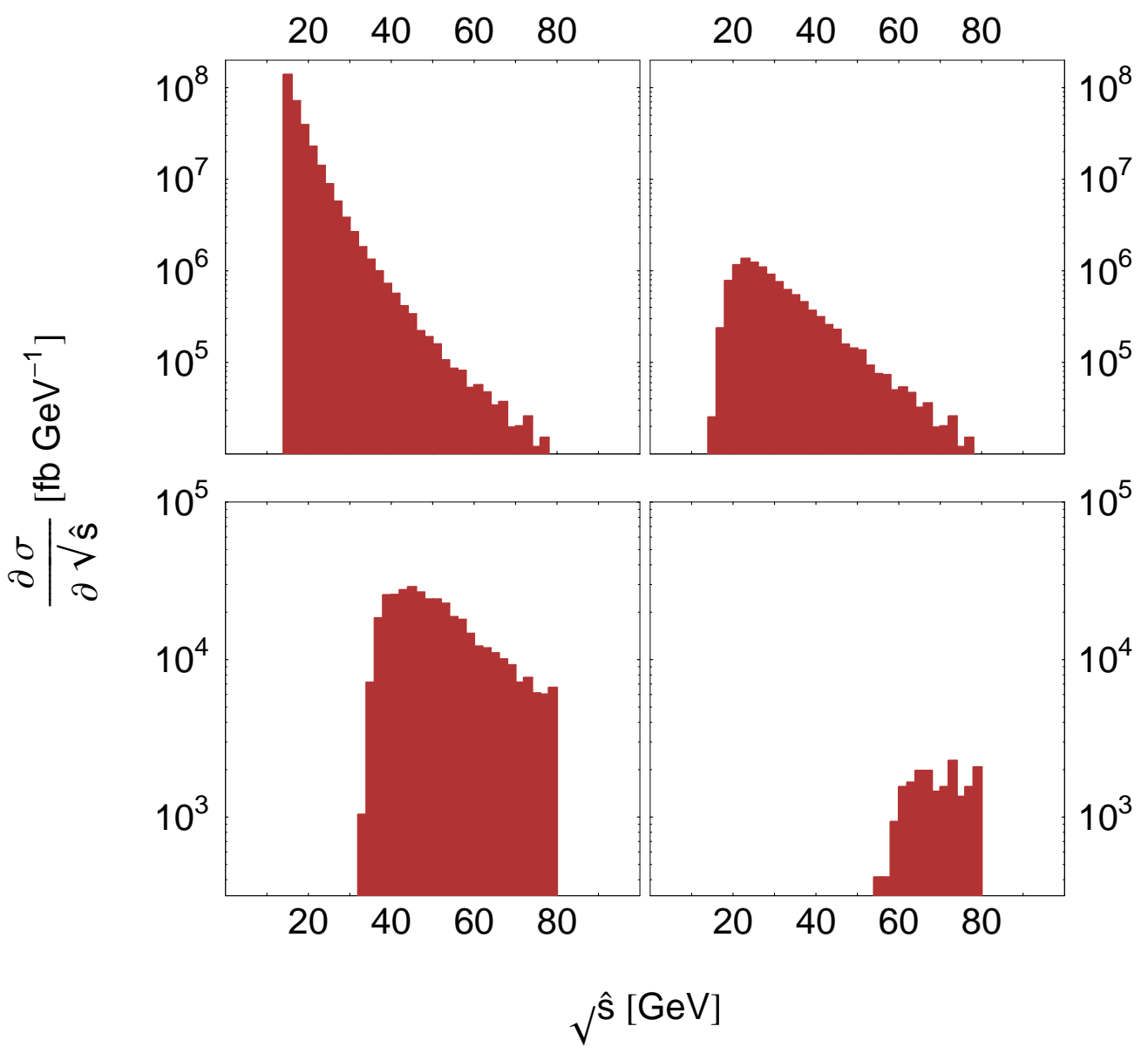

Figure 4.19: The total central mass from ExHuME di-parton events with an allowed mass range of 14 to $80 \mathrm{GeV}$. The top left shows all events, top right shows all events in which there was a jet with $E_{T}>7 \mathrm{GeV}$, bottom left shows $E_{T}>15$ $\mathrm{GeV}$ and bottom right shows $E_{T}>25 \mathrm{GeV}$.

A central mass of $15 \mathrm{GeV}$ out of a collision energy of $2000 \mathrm{GeV}$ at the Tevatron requires approximately the same $x_{1,2}$ as a central mass of $120 \mathrm{GeV}$ at the $14,000 \mathrm{GeV}$ collision energy of the LHC. It should not be surprising then that the $x$ distributions for di-jet production (shown in figure 4.21) and the rapidity coverage of the event (shown in figure 4.22) are similar to those for standard model Higgs boson production in section 4.6.1. The $x_{M i n}$ distribution for the di-jets shows a kink at around 0.0075 , roughly where the $x_{M i n}$ distribution for 


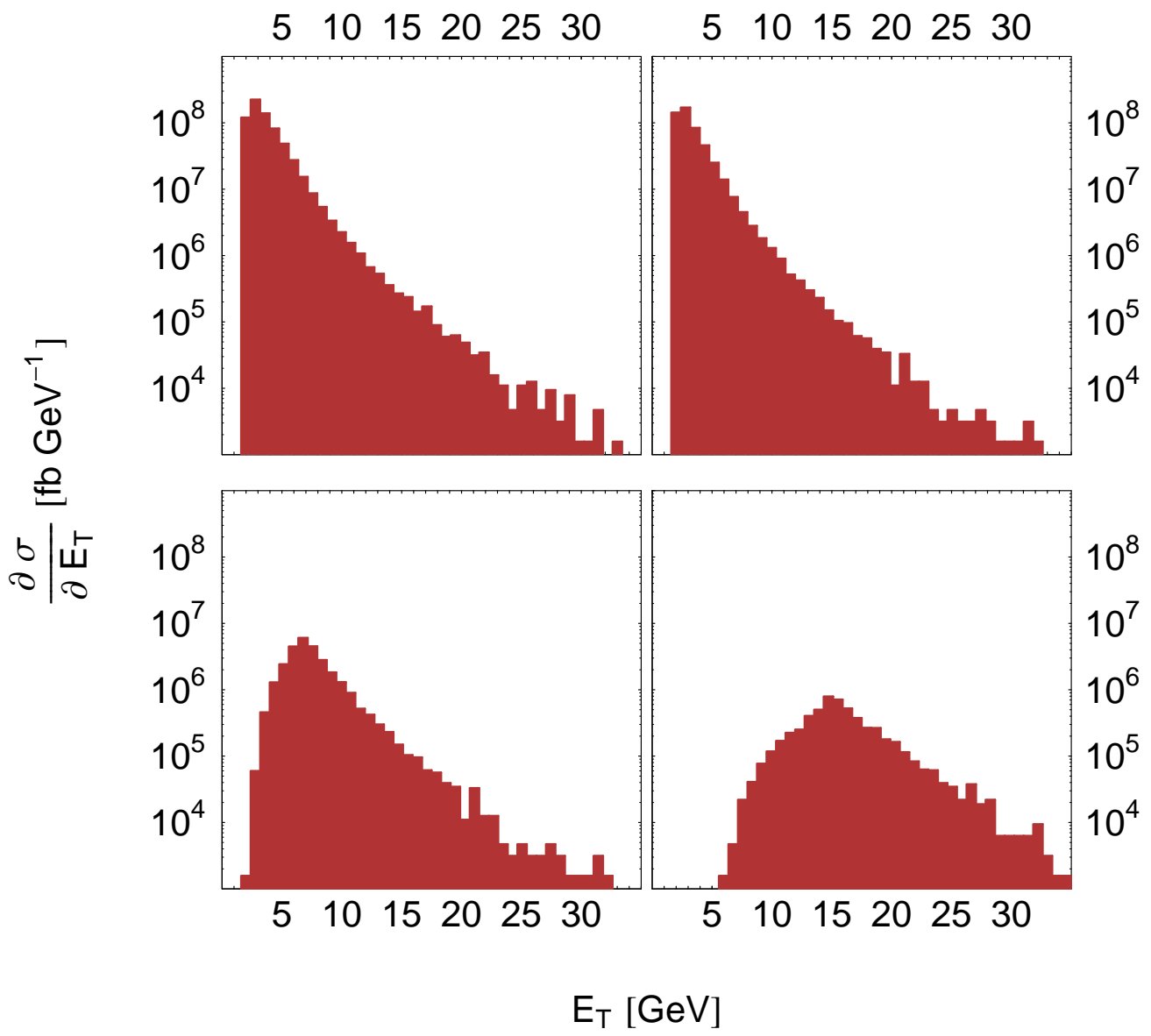

Figure 4.20: The $E_{T}$ distributions of the largest jet from ExHuME (top left) and the second largest jet (top right). Bottom left shows the $E_{T}$ distribution of the second jet when there is a cut of $7 \mathrm{GeV}$ on the $E_{T}$ of the first jet, and the bottom right shows the same with a cut of $15 \mathrm{GeV}$.

the $120 \mathrm{GeV}$ Higgs boson fades out. The difference arises because the upper limit that was set on the central mass is very tight in the case of Higgs boson production, but at $80 \mathrm{GeV}$ is loose for the di-jets. This allows a region above $x_{\text {Min }}=7 \mathrm{GeV} / \sqrt{\hat{s}}$ where $x_{1}$ and $x_{2}$ can be equal and still obey the mass cut.

The rapidity distributions of figure 4.22 show that, without a lower jet $E_{T}$ cut, there are many soft events in which the entire event is confined to within one unit of rapidity. Once the jet finder has been run and a jet $E_{T}$ cut is made, these soft events disappear. Figure 4.22 also shows that most of the cross section lies within DØ's rapidity coverage of \pm 5.2 , although a significant amount of the cross section lies outside of D $\varnothing$ 's central region of approximately \pm 3.2 that has 
full coverage.

The particle multiplicity in figure 4.23 shows that the final state particles will have an average of around $0.5 \mathrm{GeV}$ of energy. Again, cutting on the jet $E_{T}$ removes those events in which there were fewer than two jets.

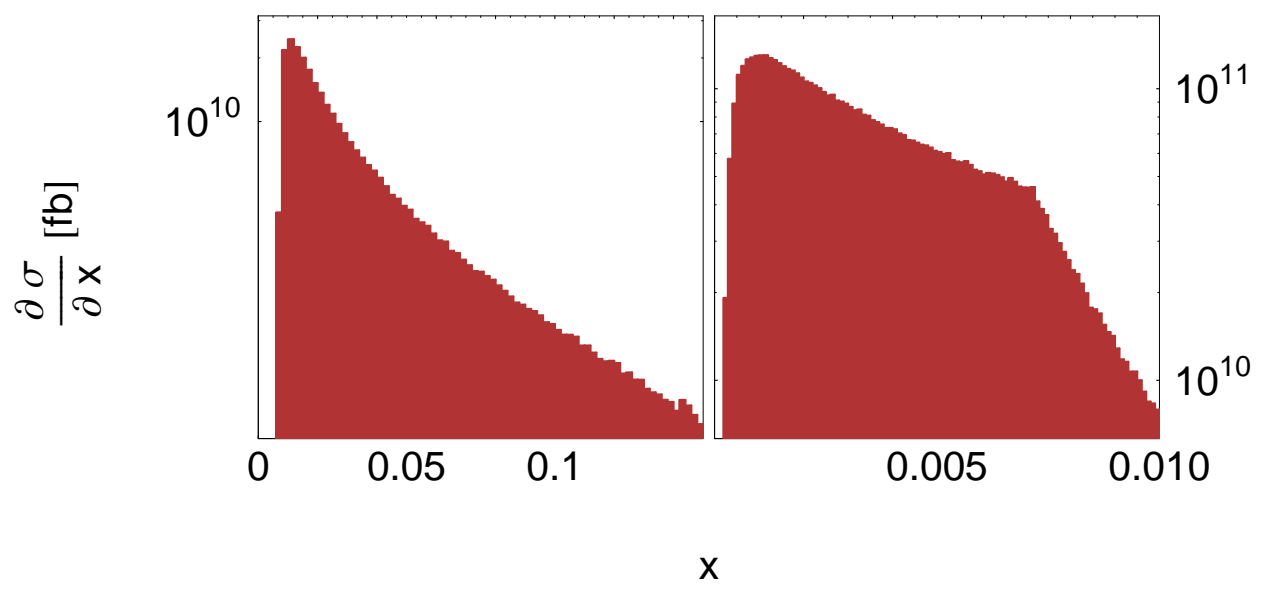

Figure 4.21: The largest (left) and smallest (right) $x$ in the event.

The di-jet mass fraction, $R_{j j}$, is defined as $M_{j j} / \sqrt{\hat{s}}$, where $M_{j j}$ is the invariant mass of the di-jet system. In central exclusive production, $R_{j j}$ is expected to approach unity. However, the jet finder does not include all of the particles in the event in the di-jet system and $R_{j j}$ will be peaked below one. This is especially true for low mass events in which the jets are soft. Figure 4.24 shows how cutting out the softer jet events forces the $R_{j j}$ distribution to the right.

With the ability to simulate central exclusive production at both the Tevatron and the LHC comes the possibility to test the simulation against data that has been and continues to be taken at the Tevatron. The remainder of this thesis describes the $D \varnothing$ experiment at the Tevatron and efforts to make the first observation of central exclusive production at $\mathrm{D} \varnothing$. 


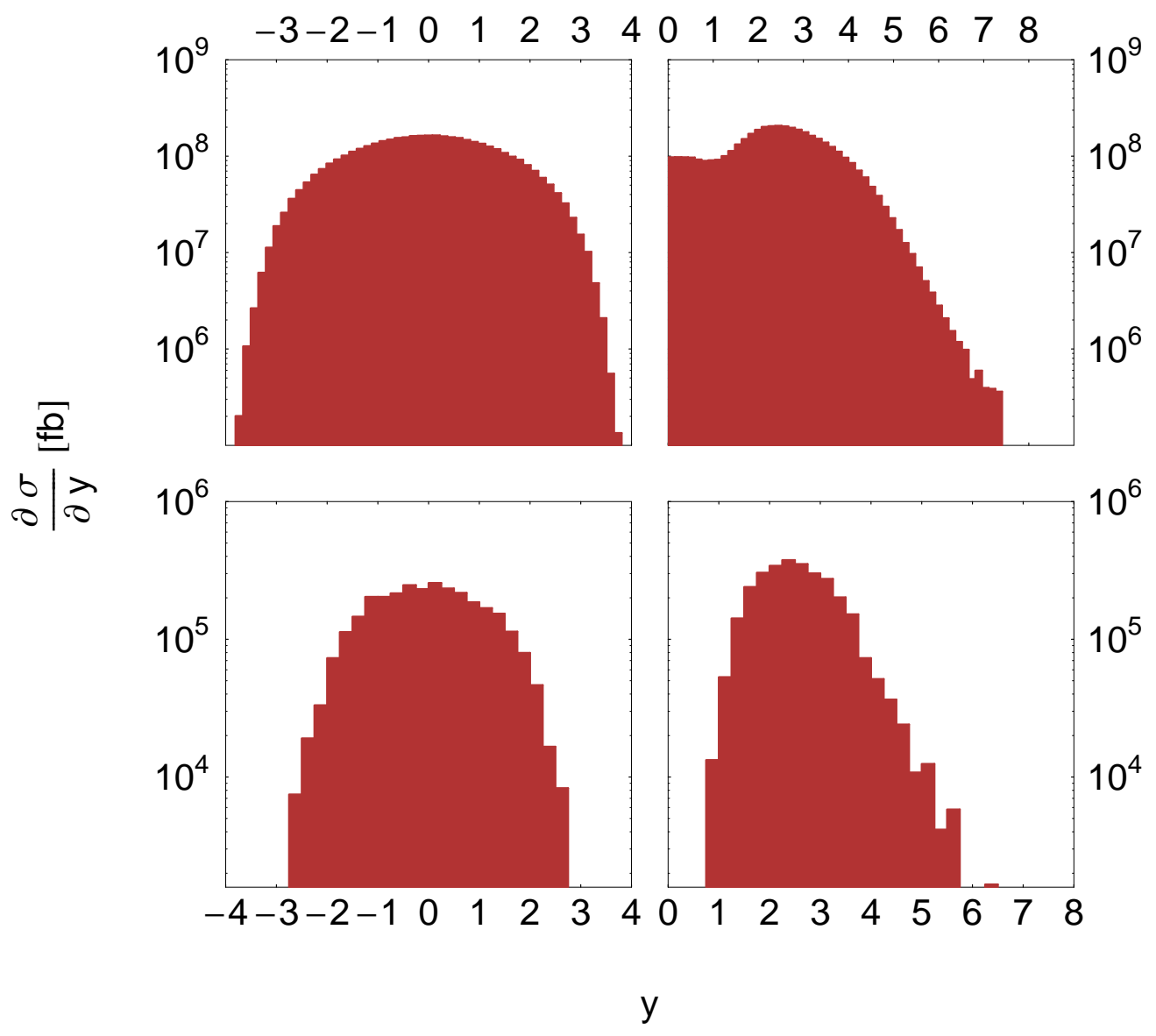

Figure 4.22: The rapidity distributions for $q \bar{q}$ and $g g$ production. The left hand column shows the rapidity of the entire central system, the right hand column shows the rapidity of the most forward particle. The top row is all events and the bottom row is those events containing a jet with jet $E_{T}>15 \mathrm{GeV}$. 


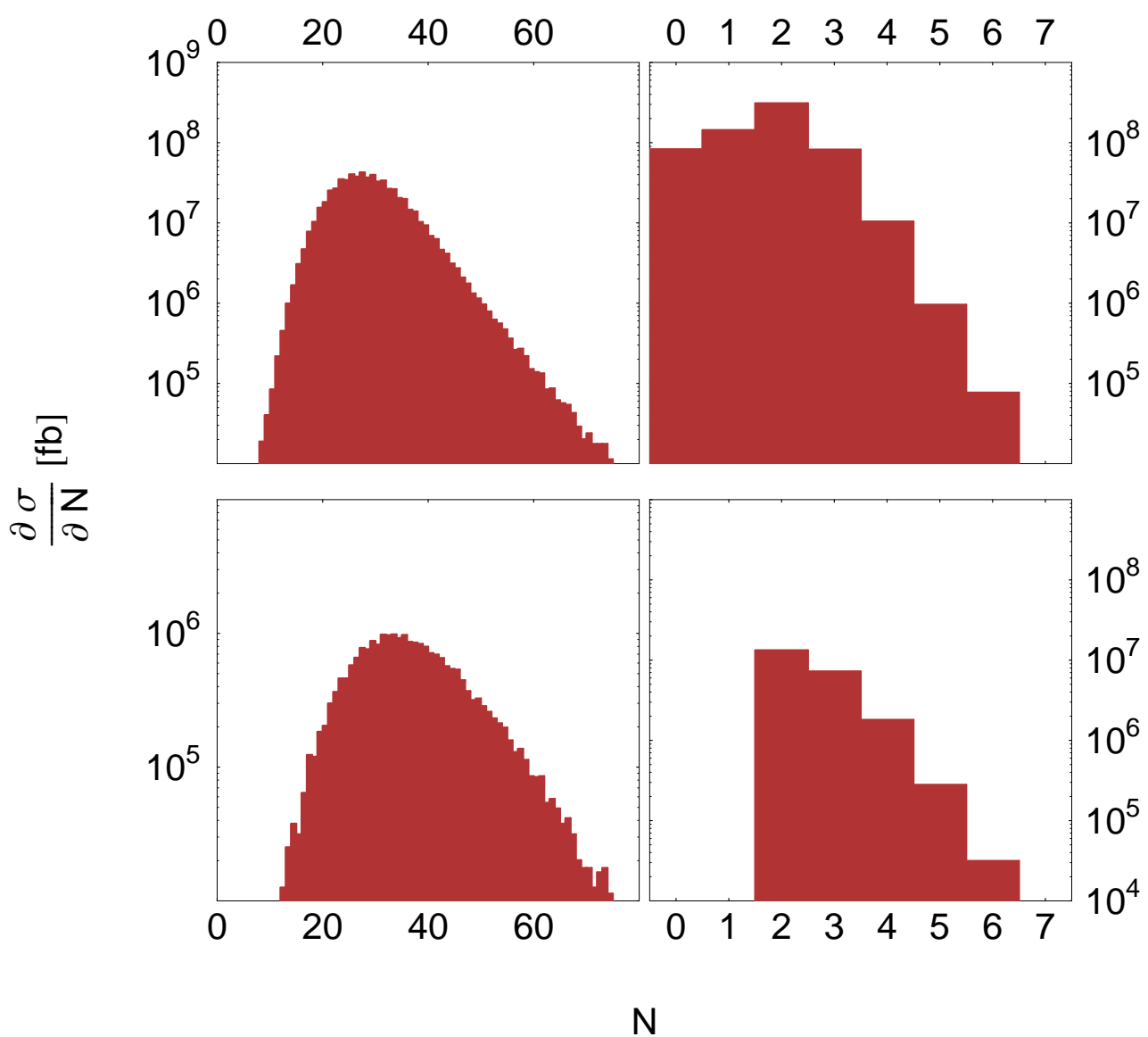

Figure 4.23: The particle (left column) and jet (right column) multiplicities. The top row is all events and the bottom row is those events with the largest jet $E_{T}>7$ GeV. 


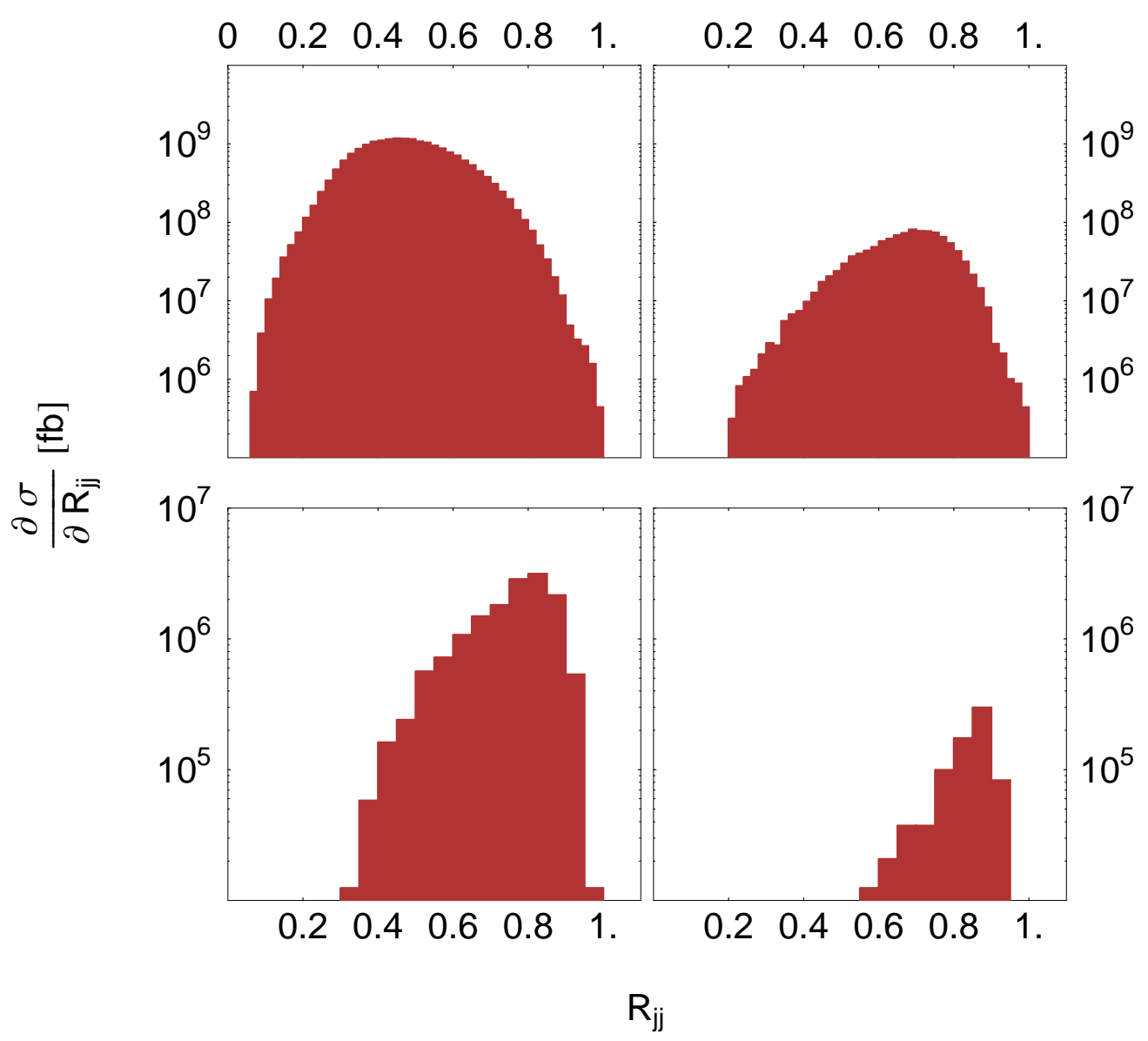

Figure 4.24: The di-jet mass fraction from ExHuME. Top left shows all events with $\sqrt{\hat{s}}>14 \mathrm{GeV}$, top right shows those events with a jet $E_{T}>7 \mathrm{GeV}$, bottom left shows $E_{T}>15 \mathrm{GeV}$ and bottom right shows $E_{T}>25 \mathrm{GeV}$. 


\section{Chapter 5}

\section{The DØ Experiment}

\subsection{The Tevatron}

The Tevatron (figure 5.1) is a circular accelerator with a circumference of approximately $6.3 \mathrm{~km}$ that provides proton on anti-proton collisions to two general purpose physics detectors on the Tevatron ring. These detectors are the Collider Detector at Fermilab (CDF) and the DØ experiment. The later was used to obtain the data used in this thesis.

Before entering the Tevatron main ring, protons are sourced from hydrogen gas that has been ionised with an electric spark. The $\mathrm{H}^{-}$ions are accelerated across a series of gradients to an energy of $400 \mathrm{MeV}$ and are also forced into bunches of around $10^{11}$ protons. The electrons are removed from the $\mathrm{H}^{-}$ions, and the resulting proton bunches are transferred to a circular booster, which accelerates them to an energy of $8 \mathrm{GeV}$. After the booster comes the Main Injector and finally the Tevatron ring itself. Anti-protons are produced by colliding $120 \mathrm{GeV}$ protons from the main injector with a nickel target. These collisions produce a spray of many different particles, most of which are pions and not the desired anti-protons. The small number of anti-protons that are produced, however, can be isolated using a magnetic field.

Protons orbit the Tevatron at a rate of around $48 \mathrm{kHz}$, steered by 1.7 Tesla magnets. The main Tevatron ring accelerates protons to $0.98 \mathrm{TeV}$, giving a centre of mass collision energy of $1960 \mathrm{TeV}$. Quadrupole focusing magnets on either 
side of $D \varnothing$ squeeze the proton and anti-proton beams so that they have a smaller cross section at the intersection point inside $\mathrm{D} \varnothing$. The time interval between bunch crossings at $\mathrm{D} \varnothing$ is $396 \mathrm{~ns}$ and there are 36 bunches of protons in the beam.

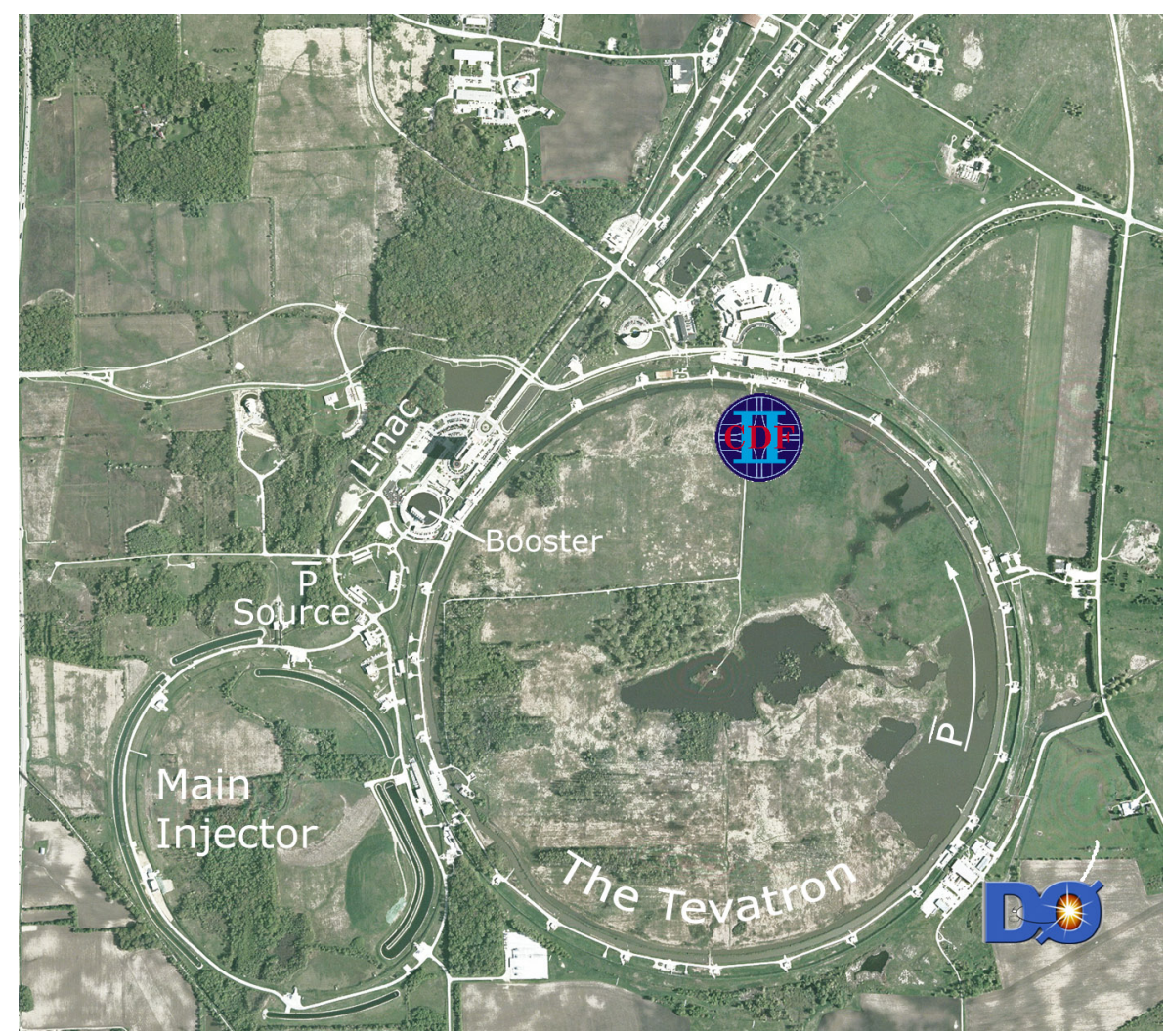

Figure 5.1: The Tevatron at Fermilab. Figure adapted from [63]

\subsection{The DØ Detector}

DØ is a general purpose physics detector with a typical "onion" structure of several detector layers, which, from the centre out are: tracking system; solenoid; calorimeter; toroidal magnet and finally the muon detector. In addition there is also a luminosity monitor between the tracker and the calorimeter. The scale of each of these components is shown in figure 5.2. The coordinate system used at $\mathrm{D} \varnothing$ is such that the protons travel in the positive $z$ direction, the $x$ direction points from the centre of $D \varnothing$ towards the centre of the Tevatron ring and the $y$ direction is straight up. For an overview of $\mathrm{D} \varnothing$ and its components during the 


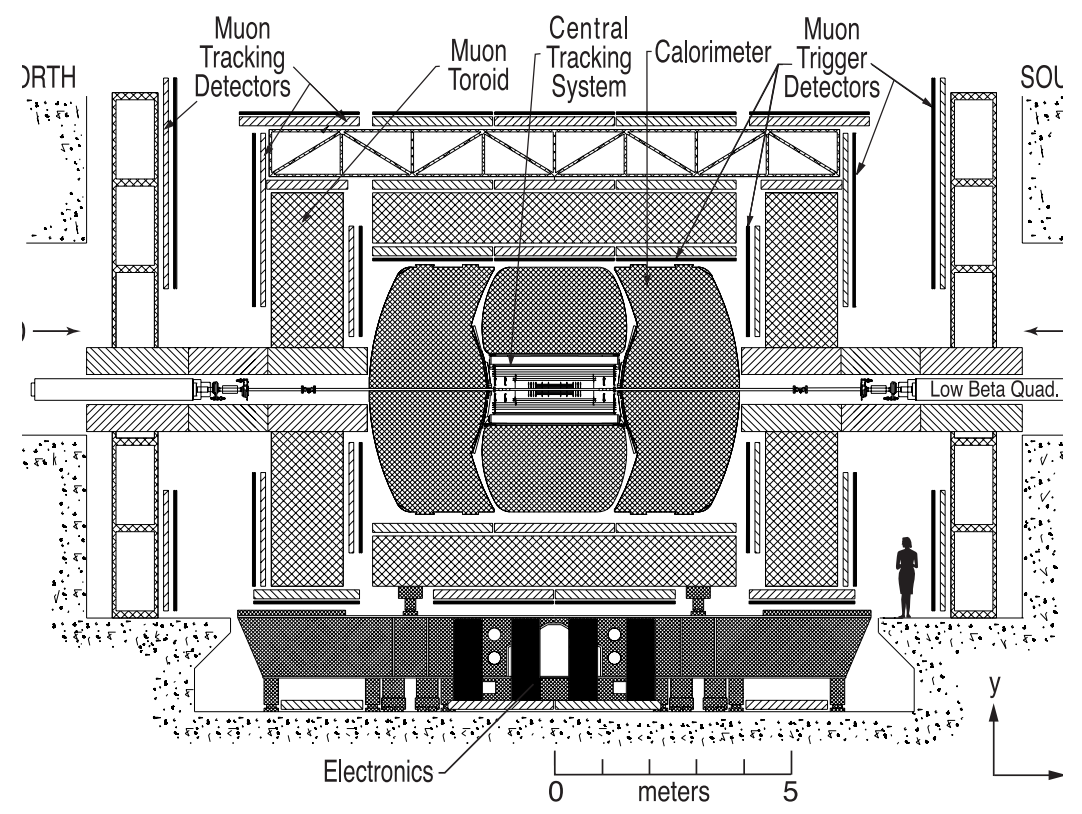

Figure 5.2: The DØ detector [64].

Tevatron run II please see [64].

\subsubsection{The Central Tracker}

The central tracker is the inner-most part of $D \varnothing$ and was designed to accurately pinpoint the tracks of any charged particles and, as a result, measure their transverse momentum. There are two trackers: an outer central fiber tracker (CFT), which produces 8 points along the track with a resolution of $100 \mu \mathrm{m}$ and an inner silicon microstrip tracker (SMT), which adds around 4 high precision points to the track and massively improves the overall track measurement giving a resolution of $15 \mu \mathrm{m}$ for a charged particle with $10 \mathrm{GeV}$ of transverse momentum. Both the CFT and SMT sit inside a 2 Tesla solenoidal magnet. Charged particles follow curved trajectories inside the magnetic field, which allows the sign of the charge and transverse momentum of the particle to be inferred from the direction and radius of the track's curvature, $R=p_{\perp} /(0.3 B)$, with $B=2 \mathrm{~T}$ and $p_{\perp}$ the transverse momentum.

The SMT consists of four concentric barrels around the beam pipe with 12 so-called F discs perpendicular to the beam. The most forward F disc is 53.1 
$\mathrm{cm}$ from the centre of the detector, and the most forward barrel is $31.8 \mathrm{~cm}$ from the centre. There are a further four larger $\mathrm{H}$ discs forward of the $\mathrm{F}$ discs at a pseudo-rapidity of approximately 2.5. Each F disc has a radius of $9.96 \mathrm{~cm}$ and consists of 12 wedge shaped double sided silicon sensors. The barrel layers are at radii of 2.72, 4.55, 7.58 and $10.5 \mathrm{~cm}$ around the beam and are composed of either single or double sided "ladders" of sensors. The inner two layers of the barrel have twelve ladders, the outer two layers have twenty four. The $\mathrm{H}$ discs have a radius of $26 \mathrm{~cm}$ and consist of 48 single sided wedge shaped sensors mounted in pairs back to back.

Silicon sensors are around $0.1 \mathrm{~mm}$ thick and consist of a layer of n-type doped silicon bonded to a layer of p-type doped silicon, or un-doped silicon for the single sided sensors. The layers of silicon are etched into thin strips, the strips in the different layers being at an angle to each other in the double sided sensors. An electric field is created across the silicon layers by applying a negative voltage to the p-type side, which annihilates some of the holes. A positive voltage is similarly applied to the n-type side. When a charged particle passes through the sensor it excites the silicon atoms to create electron-hole pairs, which are pulled apart in the electric field and collected on each side of the sensor. Since the silicon strips on opposite sides of the double sided sensors are at an angle to each other, the position at which the charged particle passed through the plane can be determined from the $\mathrm{n}$ and $\mathrm{p}$ type strip that collected the charged electron or hole respectively. There area a total of nearly $8 \times 10^{5}$ channels to read out on the SMT. For further details of the SMT please refer to [65].

The CFT has eight cylindrical layers around the beam pipe, with the inner and outer most layers having radii of $20 \mathrm{~cm}$ and $52 \mathrm{~cm}$ respectively and having a pseudo-rapidity coverage of approximately \pm 1.7 . Each layer of the CFT hold four layers of $835 \mu \mathrm{m}$ thin scintillating fiber. Two of the four layers run parallel to the beam direction, with one layer offset from the other. The other two layers are at an angle of $3^{\circ}$ to the first two layers.

When a charged particle passes through the scintillating material, photons of visible light are produced, which are counted to provide a signal. The combi- 


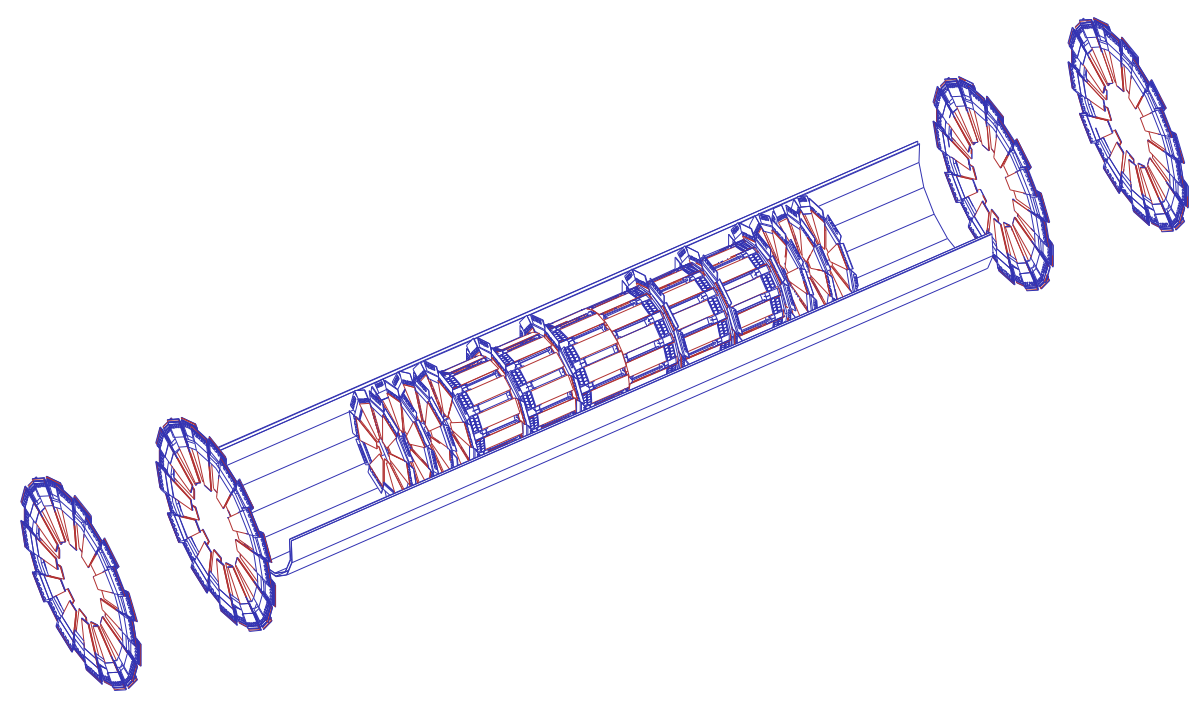

Figure 5.3: The silicon microstrip tracker [65].

nation of fibers that are lit up in each cylinder gives an intersection at the point where the charged particle passed.

\subsubsection{The DØ Calorimeter}

The calorimeter was designed to completely absorb the energy of any (Standard Model) particle that enters it (other than a muon or a neutrino) and ultimately produce an electronic signal of collected charge that is proportional to the particle's energy.

The calorimeter is composed of cells, of which there are over 60,000. Each cell contains a grounded plate made of dense absorbing material with which the passing particles interact, slow down and produce a shower of secondary particles. A readout sheet in the cell has a surface that is maintained at a high voltage (2000 Volts), which creates an electric field between the readout sheet and the grounded absorber. The shower particles leave an ionisation trail as they pass through the liquid argon in which the absorber and readout sheet are immersed. The ionisation trail drifts in the electric field, taking approximately $450 \mathrm{~ns}$ to cross the $2.3 \mathrm{~mm}$ gap between the absorber and readout sheet.

The absorber material is ${ }^{238} \mathrm{U}$, chosen for its high density and because neutrons in the showers, which would otherwise go undetected, induce fission in 
the ${ }^{238} \mathrm{U}$. During Run I, which had a bunch crossing interval of $3.5 \mu \mathrm{s}$, the charge of the fission products could be collected and used to compensate for the undetected energy of any neutrons. However, Run II has a bunch crossing interval of $396 \mathrm{~ns}$, so the charge integration time was reduced and the fission products do not arrive in time. Note also that the bunch crossing interval is less even than the drift time for the ionised trail in the liquid argon and the charge is therefore integrated over a time of approximately 260 ns, so not all of the signal charge can be collected.

The calorimeter cells are arranged into three distinct sections, each within its own cryostat that keeps the liquid argon at $90 \mathrm{~K}$; the central calorimeter covers a pseudo-rapidity range of approximately $\{-1,1\}$ and the two end calorimeters covering approximately $\{ \pm 1, \pm 5\}$. The region between the central and end cryostats contains the intercryostat detector (ICD), which uses scintillating material to give a measure of the energy lost through the intercryostat region. Within the cryostats the calorimeter cells are arranged in a tower structure of approximately constant pseudo-rapidity (figure 5.4).

Electro-magnetic (EM) showers, those arising from the interactions of charged particles or photons with the electrons in the calorimeter, are shorter than hadronic showers, which come about from the interactions between hadrons and the atomic nuclei of the calorimeter. The first four layers of the calorimeter, therefore, make up the EM calorimeter and at three to four $\mathrm{mm}$ thick have thinner absorption layers than the rest of the calorimeter. The next two to four layers form the fine hadronic $(\mathrm{FH})$ part of the calorimeter and have an absorber thickness of six $\mathrm{mm}$. The last three (one in the central calorimeter) layers compose the coarse hadronic $(\mathrm{CH})$ calorimeter and have $46.5 \mathrm{~mm}$ thick copper or steel absorption plates.

In most of the calorimeter there are 64 cells around the azimuthal axis, with each cell covering 0.1 in pseudo-rapidity. The exceptions to this are the regions forward of $\eta=3.2$, in which the cells are larger, covering a span of 0.2 in $\phi$ and between 0.14 to as much as 0.85 in $\eta$ for the most forward cells. This reduction in granularity will not, in any case, affect this analysis as, for reason explained 
in chapter 7 , events were required to have no activity forward of $\eta=3.2$. The other part of the calorimeter that has a different cell size is the third layer of the EM calorimeter, where the cells are more granular to give a better resolution of the EM jets.

The energy resolution during run I for a pion in the calorimeter as a function of the pion momentum is shown in figure 5.5.

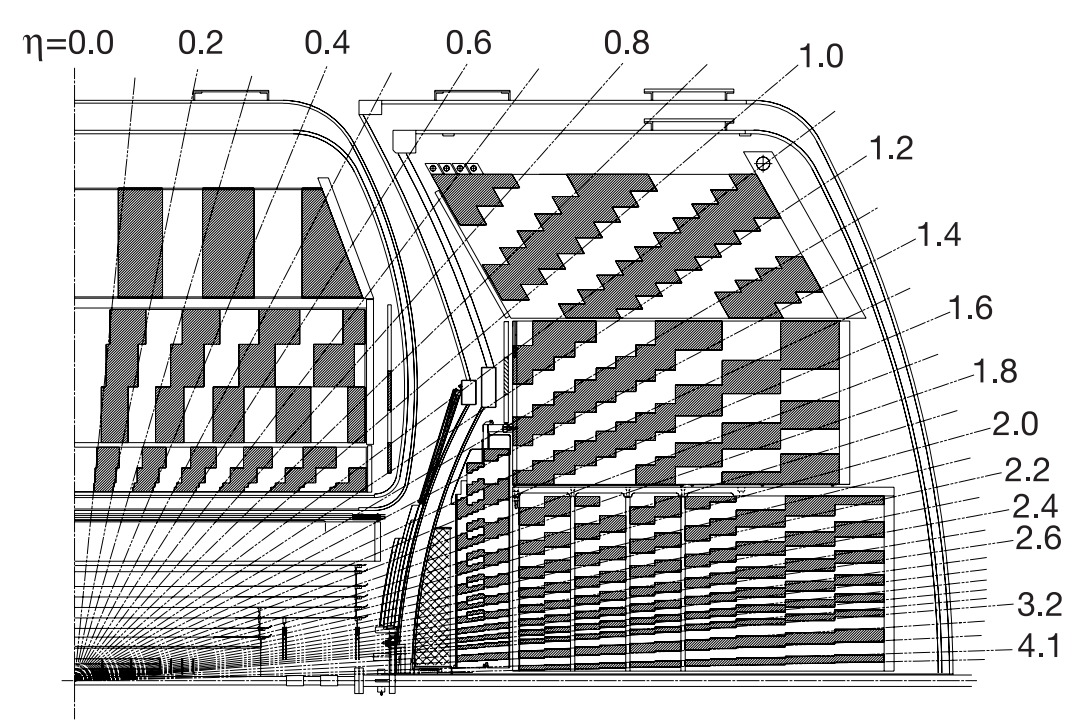

Figure 5.4: The calorimeter in cross section [64]. The interaction point is in the bottom left corner and only a quarter of the calorimeter is shown.

\subsubsection{The Luminosity Monitor}

The luminosity monitor (LM) consists of a disc of scintillating material mounted on each of the end calorimeters. An inelastic $p \bar{p}$ collision will usually result in radiation passing through the $\mathrm{LM}$, which enables the total number of $p \bar{p}$ collisions to be estimated. The total inelastic cross section is related to the forward elastic scattering amplitude, so the integrated luminosity collected can then be estimated. The LM additionally permits a so-called fastz measurement of the $z$ position of the interaction vertex from the time interval between the hits in the north and south luminosity monitors. The rate at which beam halo passes through the LM is also monitored, with so-called AHalo an PHalo bits available to record whether there was halo activity on the Anti-proton or Proton sides of DØ . 


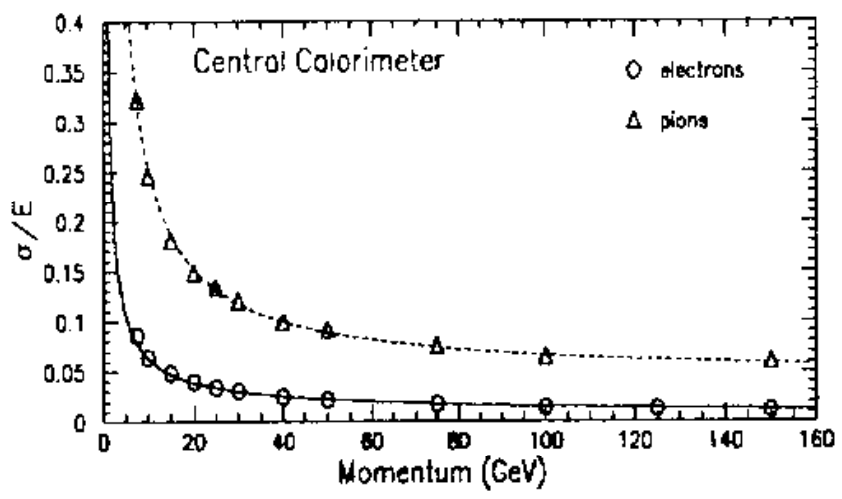

Figure 5.5: The energy resolution of the central calorimeter for a pion and an electron during Run I, taken from [66]

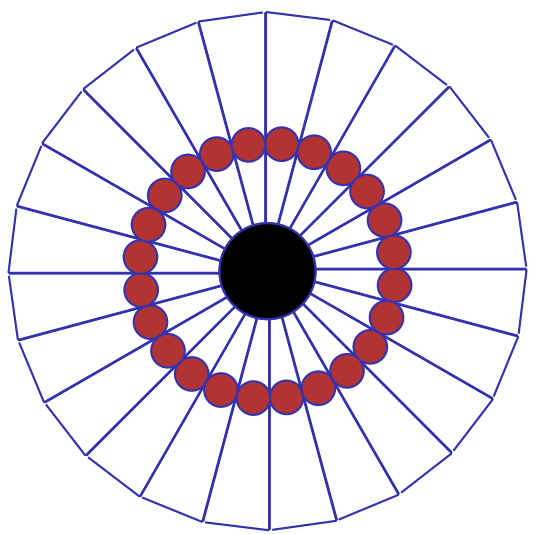

Figure 5.6: The luminosity monitor [64] showing the 24 plastic scintillating wedges, the PMTs (red dots) and the beam line in the centre.

Each LM disc has a pseudo-rapidity coverage of 2.7 to 4.4 and consists of 24 $15 \mathrm{~cm}$ long wedges at a distance of $140 \mathrm{~cm}$ from the centre of D $\varnothing$. The light produced in the wedges is detected by photo-multiplier tubes mounted on the face of the discs. Figure 5.6 shows a LM disc.

\subsubsection{The Muon System}

Unlike electrons, muons produced with energies typical to the Tevatron do not emit sufficient bremsstrahlung radiation to come to a halt within the calorimeter. At energies above $1 \mathrm{GeV}$, muons do not lose a great deal of energy to ionisation, 
either, and being colourless, do not interact strongly with atomic nuclei. A muon with an energy of around $3 \mathrm{GeV}$ is said, then, to be a minimum ionising particle, or MIP and will pass through the calorimeter. A detector is therefore required to surround the calorimeter and identify the outgoing muons.

The muon system $[64,67,68]$ is comprised of the central muon system, which has a pseudo-rapidity reach of approximately 1.0 and two forward muon systems, which cover the pseudo-rapidity region out to around 2.0. Both the central and forward muon systems consist of three layers; A, B and C, with a toroidal magnet between the innermost A layer and the middle B layer. The toroidal magnet produces a 1.9 Tesla magnetic field within its yoke, which bends the path followed by any muons passing through it. The deflection observed between the A layer and the B and C layers can be used to gain a further measure of the muon's transverse momentum, although after already having passed through the calorimeter the momentum will not be the same as at the interaction vertex.

The sensors used in the central muon system are proportional drift tubes (PDTs). The PDTs are tubes with a wire anode running through the centre and cathodes mounted on the inner sides of the tube above and below the anode. A gas composed of $84 \%$ argon fills the chamber and is continuously pumped through the detector such that the entire volume of gas is replaced three times a day (the gas is, of course, purified before being recycled back into the detector). When a muon passes through a PDT it ionises the gas, leaving the charge to be collected on the anode held at $4.7 \mathrm{kV}$ and the cathode held at $2.3 \mathrm{kV}$ over a drift time of less than 500 ns. The B and C layers of the central muon system have three decks of PDTs, while the A layer has four. There are 94 PDTs in total in the central muon system.

The forward muon system uses mini drift tubes (MDTs) instead of PDTs. MDTs work in essentially the same way as PDTs, but contain eight anode wires. As with the central muon system, there are three layers with a toroidal magnet between the innermost $A$ layer and the middle B layer.

The calorimeter needs a support structure underneath. This support structure must pass through the bottom side of the central muon system, so there is a region 


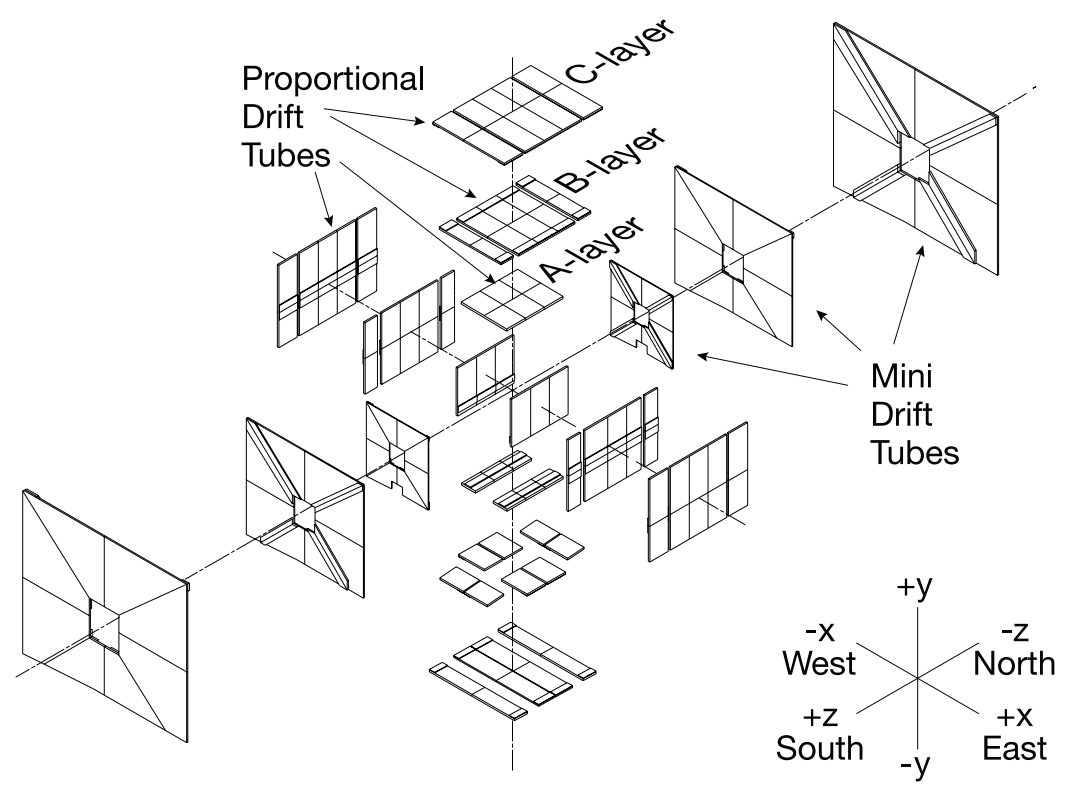

Figure 5.7: The muon tracking system [64].

without muon coverage on the underside of DØ. Figure 5.7 shows the structure of the muon system.

The muon system additionally uses scintillators [68], which are much faster to readout that PDTs, to assist in triggering and the identification of muons arising from cosmic rays striking the upper atmosphere. The central muon system has 240 scintillation counters attached to the outer-most layers on the top and at the sides and 132 counters on the underside of $\mathrm{D} \varnothing$. If a muon from a cosmic ray passes through $D \varnothing$ it can be identified if it is not in time with the bunch crossing or if the time difference between the scintillator signal on opposite sides of $D \varnothing$ is consistent with a cosmic muon. The forward muon system has a total of 4608 counters mounted on three layers at both the north and south ends of $D \varnothing$. The scintillation counters can additionally aid in the identification of beam muons that occur as a result of interactions between the beam halo and the beam pipe.

\subsubsection{Triggering}

At a bunch crossing rate of over $2.5 \mathrm{MHz}$ it is not possible to read out in full every channel from every event and write that data to tape, nor would it be desirable 
to do so, since the majority of bunch crossings do not contain any interesting physics. DØ uses a full three level trigger to decide which events are interesting and reduce the rate at which events are written to tape to around $50 \mathrm{~Hz}$.

The L1 trigger decision must either be made faster than the interval between bunch crossings, as was the case in Run I, or, while waiting for the L1 trigger decision the full data read out from every $D \varnothing$ channel must be buffered in a pipeline. Due to the increased bunch crossing rate for Run II, the level 1 trigger was upgraded to a pipelined trigger. The rate at which data is passed from the L1 trigger to the level 2 (L2) trigger is less than $2 \mathrm{kHz}$.

The L1 calorimeter trigger has available to it the total $E_{T}$ in the calorimeter as well as missing $E_{T}$ and the energy in 12 EM towers and 1280 hadronic towers. A set of thresholds exist, and if, for example, the total $E_{T}$ is greater than a threshold, or the energy of a tower is greater than another threshold then a L1 trigger bit is set. L1 bits are combined to make the decision. For instance, the JT_15TT_GapSN trigger that will be used here requires two towers with more than $3 \mathrm{GeV}$ of energy and the FastZ, pHalo and AHalo bits must be off, that is the luminosity monitor did not fire. The significance of the LM not firing is that there were no proton remnants in the forward direction so, if there was any interaction at all it was highly likely that the protons remained intact and the reaction was a diffractive or forward elastic event. The absence of activity in the forward region is a so-called rapidity gap.

The L2 trigger, having received data from L1, constructs more complicated objects. For instance, instead of using simple towers, the L2 trigger constructs simple jets from clusters of towers. The maximum rate at which events can be passed from L2 to the level 3 (L3) trigger is $1 \mathrm{kHz}$. The L3 trigger is a farm of around 100 dual cpu commodity PCs. One cpu deals with one event at a time. L3 has access to the full data read out of DØ and can execute complex software algorithms, such as the full cone jet algorithm. Many types of events occur so frequently that they fulfill the criteria to be accepted by L3 more often than the $50 \mathrm{~Hz}$ rate at which data can be written to tape. The rate of jet production, for example, is so high that the $8 \mathrm{GeV}$ jet trigger rate is too high. Many lists of trigger 
criteria therefore have a pre-scale applied, that is will only accept a fraction of the events that would otherwise be accepted. Once an event has been accepted by L3 it is written to tape for off-line reconstruction and analysis.

\subsubsection{The Forward Proton Detector System}

The Forward Proton Detector (FPD) system at DØ consists of 18 "Roman Pot" detectors - so-called because of their similarity in appearance to an ancient urn arranged into nine spectrometers. Figure 5.8 shows the layout of spectrometers.

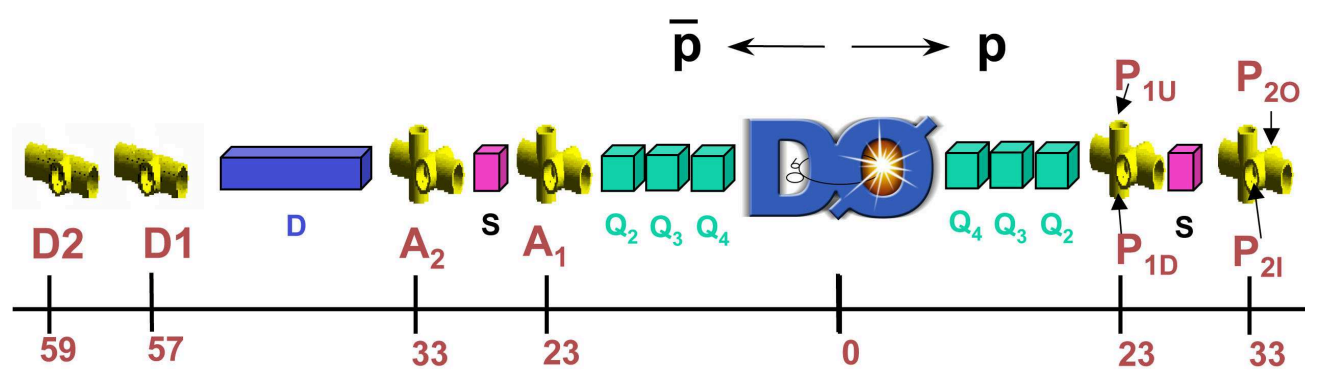

Figure 5.8: The FPD system at DØ [69]

On both the north "A side" (the Anti-protons strike the pots on this side) and the south "P" side (the Protons strike this side) there is a set of quadrupole spectrometers. Each spectrometer consists of two Roman pot detectors separated by a distance of 10 metres along the beam line, with the nearest pot at a distance of 23 metres from the centre of the $\mathrm{D} \varnothing$ coordinate system at the nominal interaction point. This distance puts the spectrometer past the three sets of quadrupole magnets that focus the beam for collisions within DØ. The effect of the quadrupole magnets on protons moving away from $\mathrm{D} \varnothing$ is to spread the beam, and protons that have been given a momentum transverse to the beam $\left(t_{1,2}\right)$ at the interaction point end up being deflected out of the beam by a small angle. If the transverse momentum and subsequent deflection is large enough then the proton will end up passing through both the first and second quadrupole pot of the spectrometer. This leaves a track that can be reconstructed to give the longitudinal momentum loss $\left(x_{1}\right.$ or $\left.x_{2}\right)$ and the transverse momentum squared $\left(t_{1}\right.$ or $\left.t_{2}\right)$. There are four 
such quadrupole spectrometers on both sides of DØ; one above and below the beam-line and one next to the beam line just inside and outside of the ring. The four sets of quadrupole pots are called A1, A2, P1 and P2, denoting the first and second sets of pots on the $\mathrm{A}$ and $\mathrm{P}$ sides.

The quadrupole spectrometers on their own do not give complete angular coverage because there is a lower limit on the transverse momentum that the proton can be given and still end up being bent into the pot. The dependence of the cross section on $t_{1,2}$ is approximately

$$
\frac{\partial \sigma}{\partial t_{i}} \simeq e^{b t_{i}}
$$

with $b=4 \mathrm{GeV}^{-2}$, so the region of most significant cross section is low $t$, the very region that the quadrupole spectrometers cannot probe. For this reason, a dipole spectrometer was also installed on the A side at a distance of 57 metres from $D \varnothing$, which is past the first beam bending magnet. The beam bending magnet has been set up to bend a proton with $980 \mathrm{GeV}$ of energy around the beam line, but any proton that has lost some energy will be bent out of the beam. The dipole spectrometer compliments the quadrupole spectrometers by providing full angular coverage on the A side.

In order to maximise the $x_{1,2}$ and $t_{1,2}$ coverage, the proton detectors should be as close to the beam as possible without causing too much disruption to the beam itself through interaction with the halo of particles that surround it. However, at the beginning of a store while the beam is being inserted into the Tevatron ring, the position and size of the beam are not stable. For this reason, the pots are attached to winding motors that can push them towards the beam or retract them to their safe home position. The usual procedure at $D \varnothing$ is to insert the pots to a position close to the beam once the instantaneous luminosity, $\mathcal{L}_{I}$, of the Tevatron has fallen to or below $\mathcal{L}_{I}=45 \times 10^{30} \mathrm{~cm}^{-2} \mathrm{~s}^{-1}\left(10^{30} \mathrm{~cm}^{-2}=10^{-6} \mathrm{pb}^{-1}\right)$. The Beams Division keep the Tevatron running at as high a luminosity as possible for as long as possible. As of September 2006, the peak instantaneous luminosity achieved during run II at the Tevatron is $\mathcal{L}_{I}=229 \times 10^{30} \mathrm{~cm}^{-2} \mathrm{~s}^{-1}$ and a store will routinely begin with a luminosity of $\mathcal{L}_{I}=70 \times 10^{30} \mathrm{~cm}^{-2} \mathrm{~S}^{-1}$ or more. This 
means that there is only a limited period of time at the end of each beam store during which the pots can be inserted into their operating positions and used to take data. Since continuous high luminosity running began in 2006, the pots have therefore not been used.

The position to which the pots can be inserted is limited by both the halo rates at $D \varnothing$ and CDF and the rate with which the pots themselves are struck by protons. As a general rule, if the halo rate increases by more than $10 \%$ then the pots are causing too much disruption to the beam, and if the scalar rate for the pots is continuously above $350 \mathrm{kHz}$ then the pots are at risk of damage. Usually the position of the quadrupole pots would be limited by the halo and the dipole pots would be limited by the rate at which they are struck. The beam position and size changes between stores (and even within stores), so the pots are not at the same position for each store. Figure 5.9 shows the outcome of positioning a (CDF) pot too close to the beam!

The proton detectors use scintillating fibres arranged in planes within a thin window of steel in the pot, which separates the scintillating material at atmospheric pressure from the ultra high vacuum of the beam pipe. The active area is $17.39 \times 17.39 \mathrm{~mm}^{2}$. There are six planes of fibres in each detector labelled $u, u^{\prime}$, $v, v^{\prime}, x$ and $x^{\prime}$. The $x$ axis runs along the edge of the window closest to the beam, with the fibres in the $x$ plane lying along the $y$ direction perpendicular to the $x$ axis. There are 16 fibres in the $x$ plane (figure 5.10) and the fibre width is around $0.8 \mathrm{~mm}$ (fibres can swell over time), which leaves a gap of $0.3 \mathrm{~mm}$ between each fibre. There are 20 fibres in the $u$ and $v$ planes lying at $\pm 45^{\circ}$ to the $x$ plane. The primed planes are identical to the unprimed planes, but are offset by one third of a fibre width. This results in 112 channels to read out per pot and a total of 2016 channels for the entire FPD system. The layout of fibres in a pot is shown in figure 5.10 .

In order to make accurate measurements of $x_{1,2}$ and $t_{1,2}$, the positions of the pots relative to the beam are needed. The next chapter explores the possibility of using the distribution of proton hits themselves to determine the location of the beam with respect to each pot. 


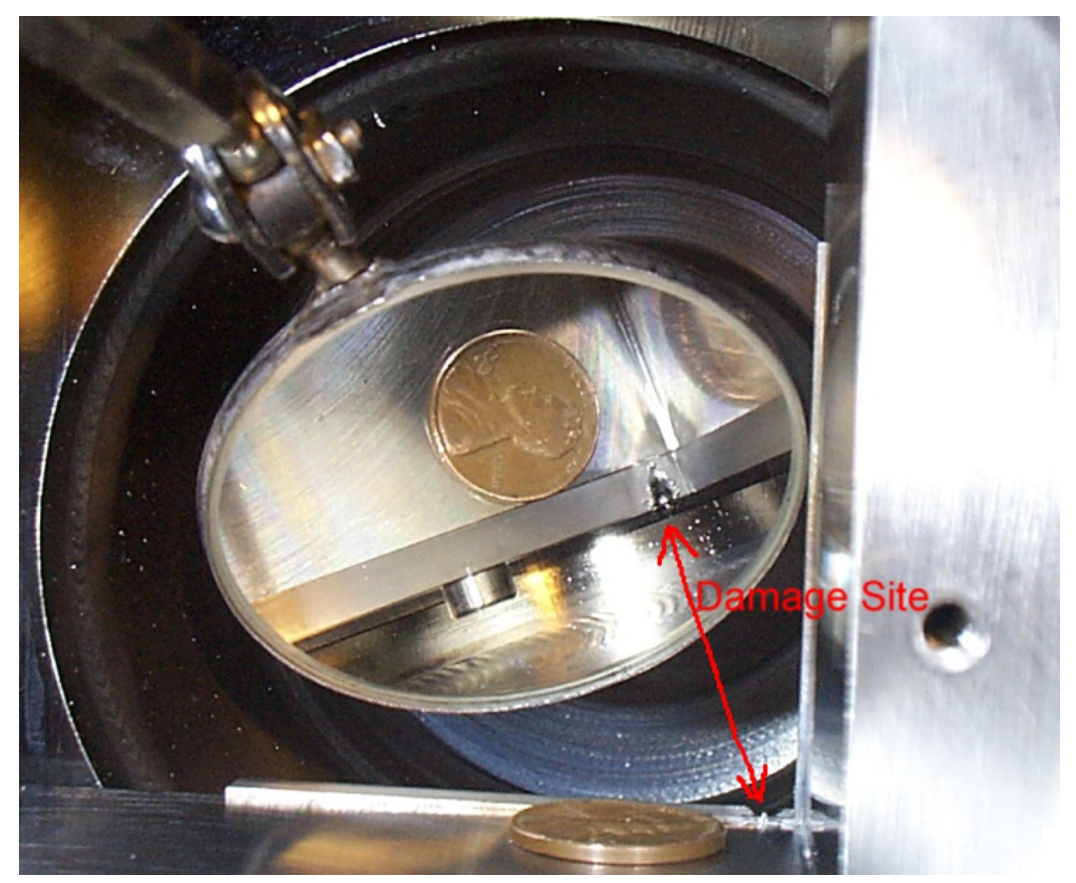

Figure 5.9: Photograph of damage caused to a Tevatron beam collimator [70]. On the 5th of December 2003 there was a malfunction of a CDF pot, causing it to move from its safe home position to a point beyond its maximum safe inward position. The pot did not actually collide with the beam, but interactions with the beam halo caused a shower of particles that heated the nearby super-conducting magnets, which quenched. The loss of steering magnets caused the beam to hit and bore a hole through the tungsten collimator target shown, as well as melt a hole several centimetres deep through a much thicker steel collimator. The resulting heating and deposition of beam energy around the Tevatron ring caused sixteen out of the twenty eight Tevatron stations to quench - the largest quench ever experienced at the Tevatron. 

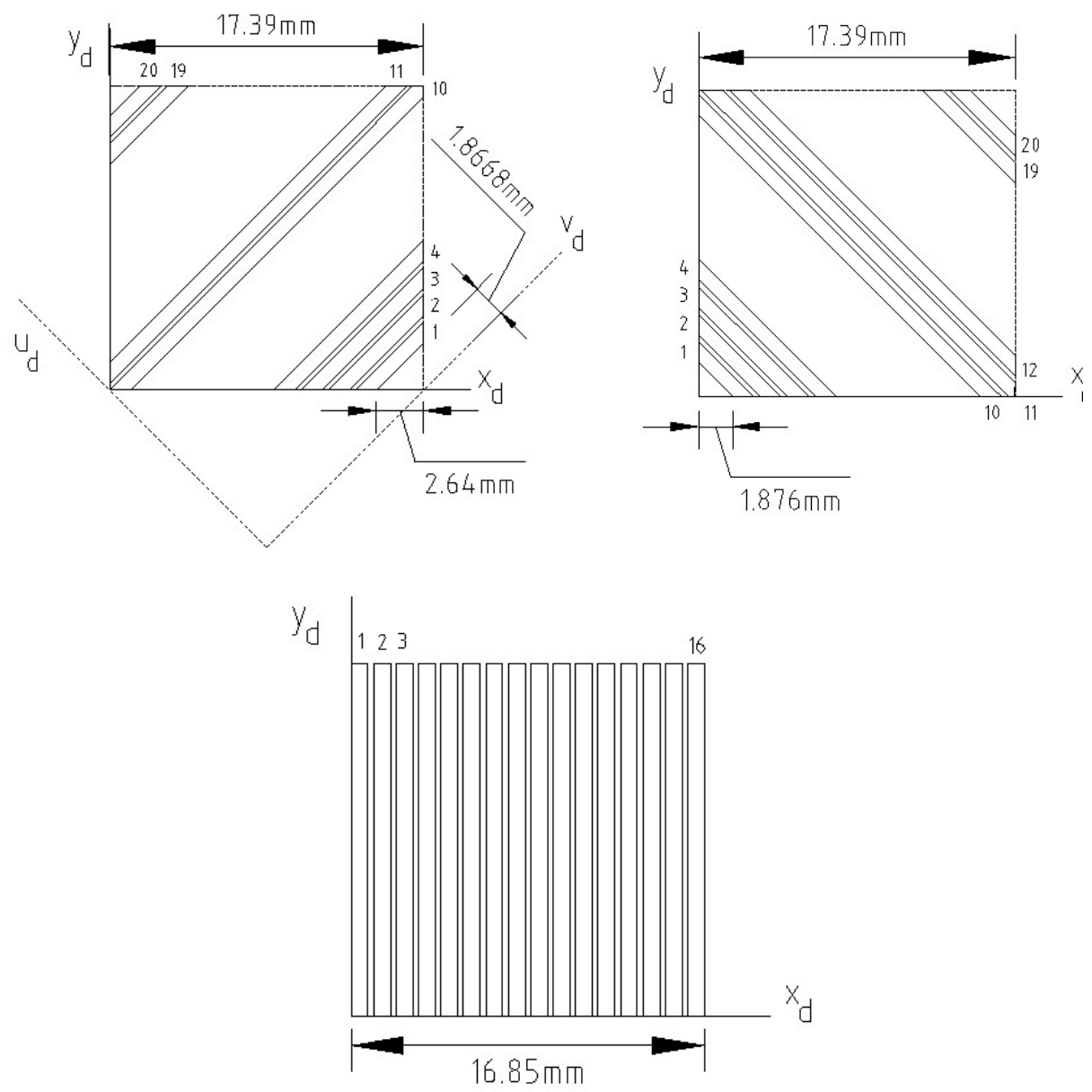

Figure 5.10: The $\mathrm{u}$ (top left) $\mathrm{v}$ (top right) and $\mathrm{x}$ (bottom) planes of fibres [71]. 


\section{Chapter 6}

\section{Proton Hit Patterns in the Forward Proton Detectors}

Typically, the positions of the forward proton taggers would be determined by fitting the data to the elastic $p \bar{p} \rightarrow p \bar{p}$ peak in the cross section. However, elastic collisions produce nothing in the central detector that can be used to trigger the detector, so either a special run of data taking must be made or the FPD system itself must be able to trigger the electronics. This could prove especially problematic for the proposed FP420 system [30] at the LHC because the decision to trigger in the central detector lies outside of the light cone of the corresponding hit in the proton taggers at $420 \mathrm{~m}$. Similarly, at DØ, a large amount of data was taken when the pots were present, but no trigger was available for them. It would therefore be desirable to have a method for obtaining the pot positions without needing to trigger on proton hits in the proton taggers.

The hope in this analysis was that the distribution of (anti)proton hits in the pot window could be used to determine how far the beam was from the pot. The beam is roughly elliptical in cross section and so should be surrounded by elliptical contours of approximately equal proton flux. Over the course of a run at $D \varnothing$ this should be revealed in a pot window as a set of contours along which the density of proton hits is constant. One would expect many such contours and by fitting curves to all of them it might be possible to determine the position of the centre of the beam line from the centres of the elliptical curves. 


\subsection{Reconstruction of the Proton Hit Positions}

The raw fibre hit information from the pots is available in a ROOT [72] file format, from which the proton hit positions had to be reconstructed. To discriminate between fibres that are on and fibres that are off a pedestal file is needed. A pedestal for each fibre channel is determined by measuring the amount of signal charge collected in one bunch crossing interval when there is no beam in the Tevatron. The pedestal file contains the average charge and the standard deviation of the distribution of charges collected for each fibre and can be extracted from data files in the $D \varnothing$ online system.

The raw fibre readings were compared to the pedestal and if the reading was 4 standard deviations or more above the pedestal average then the fibre was declared to be on. If more than five fibres were on in a single pot layer then the pot was deemed to be bad for that event and the entire pot was said to be off and was ignored. Five fibres could be lit up either because a large number of halo protons passed through the pot window or simply because of random noise. In any case, such an event would contain so many hits in the pot that it would be useless. A map was used mapping the fibres' numbers to their positions in the plane. Mapping corrections were needed to account for differences between the fibres' ordering in the data file and their physical ordering in the layer.

Having determined the positions of the lit fibres, the intersections of those fibres were found as follows: if the intersection is between $\mathrm{a} u$ and $\mathrm{a} v$ fibre then the $x$ and $y$ coordinates in the pot window are given by

$$
\begin{aligned}
& \mathrm{x}=\frac{1}{2}(-\mathrm{u}+\mathrm{v}) \\
& \mathrm{y}=\frac{1}{2}(\mathrm{u}+\mathrm{v})
\end{aligned}
$$

whereas if the intersection is between $\mathrm{a} u$ and an $\mathrm{x}$ fibre then:

$$
\mathrm{y}=\mathrm{x}+\mathrm{u}
$$


or if the intersection is between a $v$ and an $\mathrm{x}$ fibre then:

$$
\mathrm{y}=-\mathrm{x}+\mathrm{v} .
$$

It is possible for several intersections to arise from a single proton, either because of the overlapping fibres in the primed and unprimed planes or because a proton could pass through a $u, v$ and $x$ fibre to leave a triangle of close intersections (figure 6.1). Any intersection points lying within $1.62 \mathrm{~mm}$ of each other (approximately two fibre widths) were therefore merged recursively to form a single point at the average position.

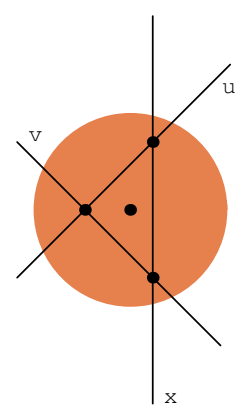

Figure 6.1: The intersection of three nearby fibres that are merged to form a single hit at the centre of the circle.

Over the course of a run the position of each proton hit in each pot window was recorded and a density plot of the proton hits was made (figure 6.2). The number of protons hitting a region of the pot window should be proportional to the proton flux through the pot at that point, so figure 6.2 contains an image of the beam halo profile together with the diffractive signal distribution.

The dipole pot windows show a clear structure of a lobe of (red) proton flux poking into the pot window from the edge nearest the beam on the left hand side. This oval profile approximately corresponds to the shape of the beam. The quadrupole spectrometers, however, show no such obvious structure to the pattern of hits, either because of noise or the much larger halo rate in the quadrupole spectrometers. Figure 6.2 shows three images overlaid: the hits from 
the diffractive signal, the beam halo and detector noise. Each contribution may produce a distinct structure in the pot. There will normally only be at most one signal proton produced on each side per bunch crossing, so if there are two or more hits in a single pot in a single event then at least one of those hits is caused by either beam halo or noise. Conversely, those events in which there was at most a single hit in each pot should have an enhanced contribution from the diffractive signal distribution over the beam halo distribution. Plotting the proton flux for only those events in which there was a single hit therefore improved the definition of the lobe in the dipole pots (figure 6.3). No structure was revealed in the quadrupole pots. 


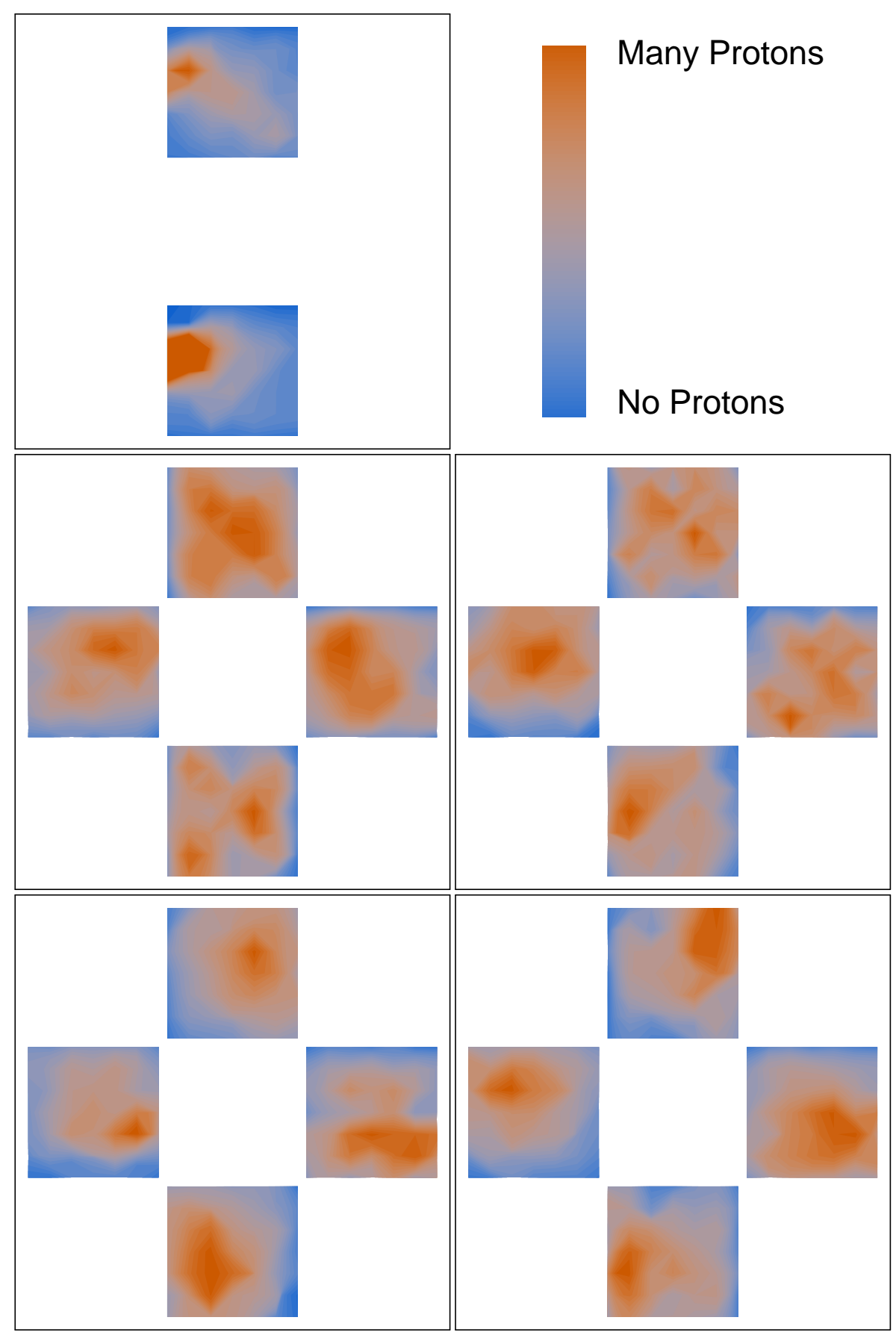

Figure 6.2: Proton flux patterns in the Roman pots. Left hand column shows the A side spectrometers, with the dipole at the top and A2 at the bottom, and the right column shows the P side quadrupole spectrometers, with P2 beneath P1. 

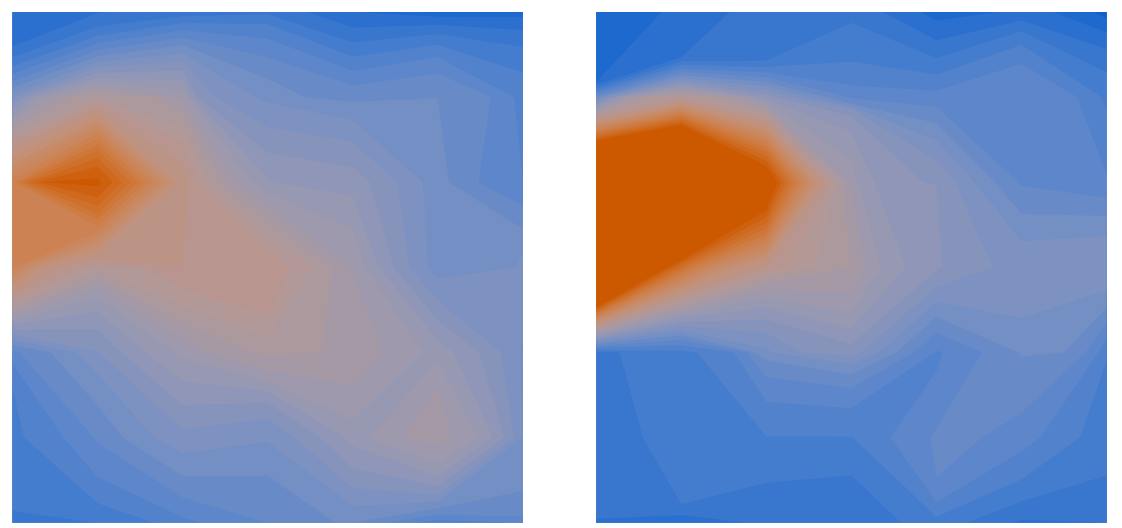

Figure 6.3: Proton flux in the D1 dipole pot window. The left hand density plot shows all proton hits; the right hand plot shows those hits from events that contained only a single pot hit. There is a clear enhancement to the definition of the image of the lobe structure of the proton flux in the right hand plot.

\subsection{Least Squares Fitting of an Ellipse}

The oval shaped beam profile visible on the left hand side of the pot windows in figure 6.3 raises the possibility that analysis of such images may provide an alternative method to fitting the elastic $p \bar{p} \rightarrow p \bar{p}$ peak for locating the position of the beam in relation to the pot window. Naively, one expects the beam profile to be a circle centred on the nominal centre of the beam. Beam magnets, collimators and separators modify the shape of the beam so it is not circular, so the next simplest beam shape is an ellipse centred on the nominal beam centre. Finding the centre of an ellipse fitted to the flux distribution of figure 6.3 therefore gives a measurement of the position of the beam in relation to the pot, which is crucial in making accurate measurements for $x_{1,2}$ and $t_{1,2}$.

The pot window was divided up into a grid of $10 \times 10$ square bins, each of side $1.739 \mathrm{~mm}$. After finding the proton hits for each event over the course of a run, the number of hits in each bin was counted (figure 6.4). A run typically contains around 100,000 events, but after selecting only those events in which there was a single pot hit only around 5000 events would remain. The set of points $\{x, y\}$ lying on an ellipse obey the equation 


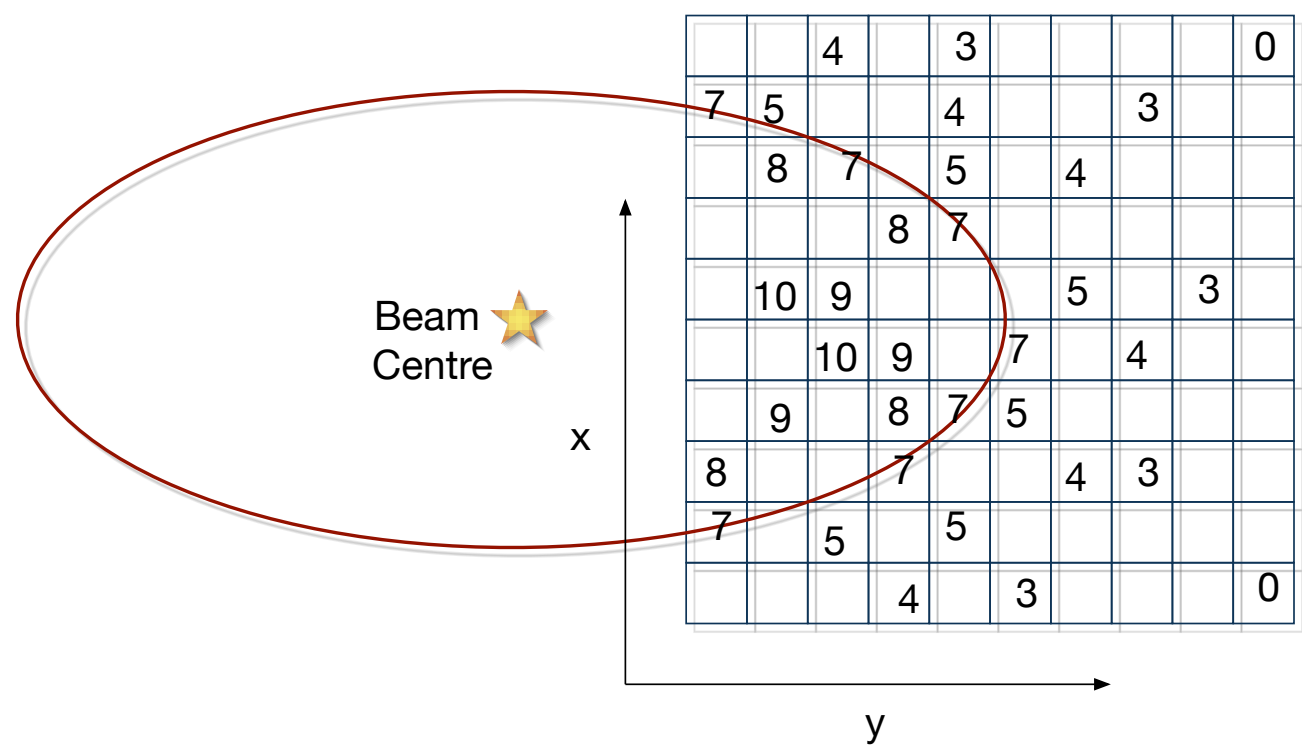

Figure 6.4: A schematic of the pot window divided into bins showing the number of proton hits in each bin. An ellipse has been fitted through those bins with 7 hits and the centre of the beam lies at the centre of the ellipse. There are many such elliptical curves that could be fitted through points with equal hit rate.

$$
\left(x-x_{0}\right)^{2}+e^{2}\left(y-y_{0}\right)^{2}=r^{2}
$$

where the centre of the ellipse is at $\left\{x_{0}, y_{0}\right\}, e$ is the eccentricity of the ellipse and $r$ is the length of one of the axes. The ellipse of equation 6.4 has its axes parallel to the $x$ and $y$ axes of the coordinate system, so in general there is one more degree of freedom to rotate the ellipse. A minimum of five points with the same number of hits is therefore needed to define an ellipse, and in fact the method used here requires six points [73], yet very few of the bins contained precisely the same number of hits as another bin.

The solution is to interpolate points between the centres of adjacent bins. So, for example, if a bin with 10 hits lay next to a bin with 13 hits then points would be generated at one third and two thirds of the way along the line joining the centres of the two bins, corresponding to points with 11 and 12 hits. In this 


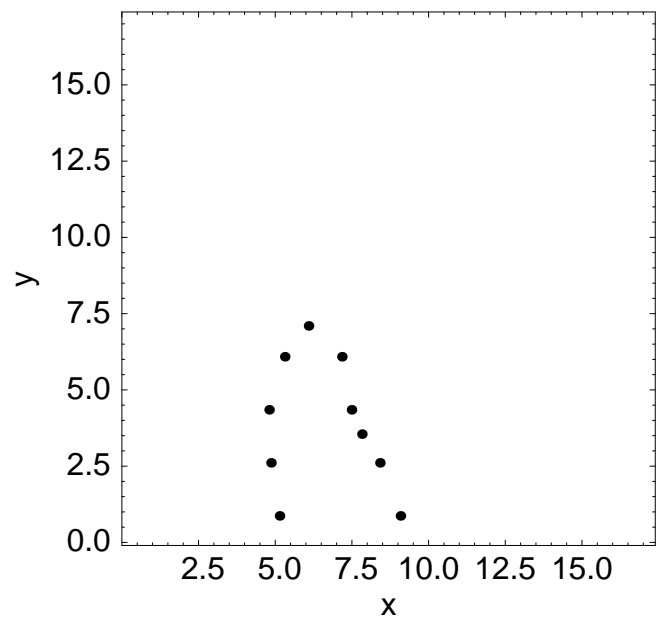

Figure 6.5: A set of points in the pot window all with the same proton flux.

way, many sets of points in which each point has the same number of hits was generated. An ellipse may be fitted to each set that contains six or more points. One such set of points to which it is possible to fit an ellipse is shown in figure 6.5 .

The ellipses were fitted using the method of direct least squares fitting described in [73] that involves finding the eigenvectors of a $6 \times 6$ "constraint" matrix. This method has the advantages that it is fast (it is not an iterative method) and is guaranteed to converge upon a bounded elliptical solution for any set of six or more points. Figure 6.6 shows some ellipses that have been fitted to ten points chosen at random using the $\mathrm{C}++$ implementation that was written for the fitting of the pot hit data. When the data points lie exactly on an elliptical path (left plot) the fitting routine works perfectly to recreate that curve, even if the data points only lie on a small section of the ellipse. The middle and right hand plots of figure 6.6 show ellipses that are fitted to points that have been smeared randomly by $\pm 10 \%$ from a true elliptical curve. So long as the points are distributed all the way around the ellipse (middle plot) the fit is good. If the points are clustered towards one end of the ellipse (right-hand plot) and are also smeared then the fit tends to underestimate the true eccentricity of the ellipse as a result of enforcing a closed loop. This has consequences for the pot calibration because the pot can 
only ever sample a small section of the beam profile.
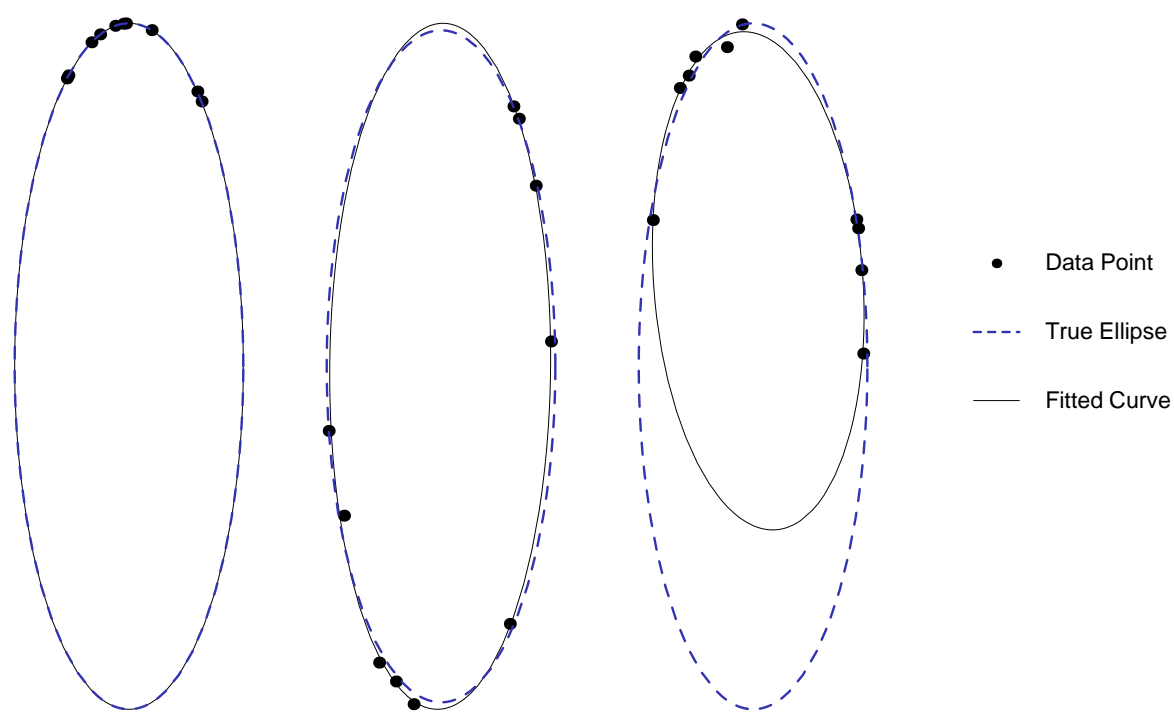

Figure 6.6: Ellipses fitted to ten smeared points. The left hand figure shows points that lie exactly on an ellipse, and the resulting fitted curve exactly matches the true ellipse, even though the points only lie along a small section. The middle figure shows points that have been smeared about an ellipse, but which nevertheless lie all around the ellipse. The middle curve is a good fit to the true ellipse. The right hand figure shows smeared points that lie only in the upper half of the ellipse. The fitted ellipse underestimates the eccentricity of the true ellipse in this last case.

To eliminate some of the low eccentricity fits made to noisy data points, ellipses were only fitted to sets of points for which the number of proton hits was five or more. Further, and any ellipse with an eccentricity of less than two was discarded. Any ellipse with a centre that lay inside the pot window $3 \mathrm{~mm}$ or more from the edge closest to the beam was also discarded because the beam itself should never pass through the pot window.

A Monte Carlo sample of pot fibre hits in the dipole spectrometer from 10,000 events was provided using the simulation in [74]. The simulation used the 2003 positions and strengths for the beam magnets, compared to the data which was taken in 2004. The difference in magnet strengths and positions explains the difference in shape between the proton flux image for the simulation (figure 6.7, left-hand plot) and the data (figure 6.2). The proton hits in the first dipole pot 
were reconstructed as in section 6.1 using the same code as used for the data and the ellipse fitter was run on the resulting points. Two distinct families of ellipses were visible in the Monte Carlo sample. One set was almost circular and contained almost entirely within the pot window. A second set had a much greater eccentricity and extended outside the pot window. The first of these sets was attributed to fits to a small number of points near the edge of the pot window and, given that they lay entirely within the pot, could not be related to the position of the beam. To distinguish between these two groups of ellipses, any ellipse with an eccentricity of less than two or with a centre that lay more than $3 \mathrm{~mm}$ inside the pot window $3 \mathrm{~mm}$ was discarded.

After selecting on eccentricity, a set of 102 elliptical curves was found that satisfied all requirements. The ellipses with the largest and smallest eccentricities are shown in figure 6.7, together with the proton flux through the pot window. Since the direct least square fit method tends to underestimate the eccentricity of the true ellipse, the most eccentric ellipse found should give a lower limit to the eccentricity of the true ellipse. This also means that the beam is further from the pot than the centre of the most eccentric ellipse.

The beam in the Monte Carlo simulation lay at a distance of $8.72 \mathrm{~mm}$ from the edge of the pot window, which is shown by a red line. The centre of the largest ellipse, shown with a black dot, lay $8.66 \mathrm{~mm}$ from the pot window. The $x$ coordinate of the centre of the ellipse was $10.55 \mathrm{~mm}$.

The ellipse fitting method can provide a useful limit on the position of the beam under the ideal noise and background free conditions of a Monte Carlo sample. In contrast, real data contains both noise and halo background. The code was applied to the raw fibre data for 66,854 events from run 195802, selected because of its typical number of events; if the method was to work for most runs then it would have to work for this one. The resulting proton flux plot and ellipses are shown on the right in figure 6.7.

The fit to the data has not produced a reasonable position for the beam, with the centre of the most favourable ellipse lying $1.00 \mathrm{~mm}$ inside the pot window. There are two major causes of this: unavoidable noise in the detector smears 


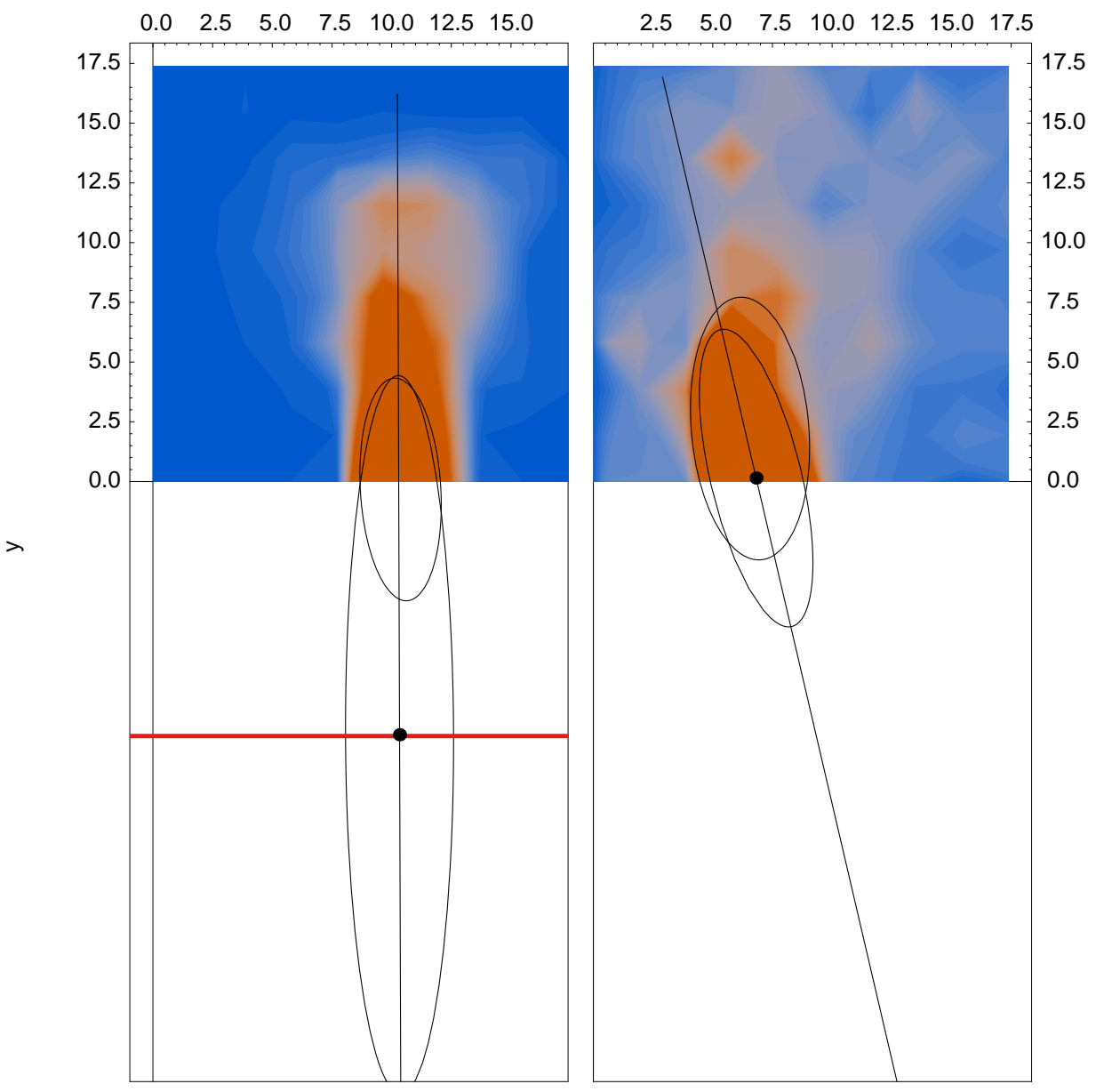

Figure 6.7: Ellipses fitted to Monte Carlo sample of diffractive pot fibre hits (left plot) and fibre hits from data (right plot). In the Monte Carlo plot the most eccentric ellipse is also the one with its centre furthest from the pot. The centre of this ellipse (black dot) coincides almost exactly with the beam position (red line). The centre of the ellipse shown in the right hand plot was the furthest from the pot of any of the ellipses found. The least eccentric ellipse found is also shown in both cases. 
the data points to which the ellipses are fitted and results in an underestimation of the semi-major axis length; and the data contains beam halo and diffractive signals overlaid. The beam halo lies in a "halo band"; halo protons tend to have correlated coordinates in both pots in the spectrometer because halo protons travel parallel to the beam, whereas signal protons travel at an angle to the beam. The halo protons will then reveal themselves in a scatter plot of $y_{1}$ versus $y_{2}$ as a correlation band, where $y_{1}$ is the $y$ position of the hit in the first pot and $y_{2}$ is the y position of the hit in the second pot. Rejecting hits which lie near the halo band (or only taking hits from within the halo band) will then select the signal (or halo) ellipses. Alternatively, it may be possible to extract the halo directly from the proton flux plots of figure 6.2. It is unlikely that two signal hits would occur in the same event, so any event with two hits almost certainly contains one beam halo or noise hit and one hit which is either diffractive signal, noise or halo. By subtracting the image for events with only a single hit from the image for two hits it should be possible to arrive at the image left in the pot by the halo and noise alone.

It is also possible to improve the ellipse fitting algorithm so that it is less sensitive to noise. A method for reducing the low eccentricity bias of the elliptical fits is given in [75], which takes the direct least square fit as a starting point and improves upon it iteratively.

To summarise, it has been shown that density maps of the proton flux through the pot window can be made and that, for the dipole pots at least, there is a visible structure to the proton density that corresponds to the profile of protons around the beam. Ellipses were fitted to contours of equal proton density in a sample of simulated events and data. In the simulated events it was found that, no matter which contour was used to fit the ellipse, the centre of the fitted ellipse always lay closer to the pot than the true centre of the beam line. The fitted ellipses did, however, follow the visible contours of equal proton flux within the pot and the ellipse with the centre furthest from the pot did approach the beam centre. In the simulated events, then, the fitted ellipses can be used to provide a point that is always closer to the pot than the nominal beam centre. The major 
axis of the fitted ellipses also pass through or close to the centre of the beam. In the data, however, the fitted ellipses are biased towards lower eccentricity due to smearing of the number of proton hits. It is therefore still true that the centre of the fitted ellipse is never further from the pot than the centre of the beam, however, in this case the ellipse centre lay inside the pot, which clearly cannot have been the beam position. Much more analysis and development would therefore be needed to account for the smearing and low eccentricity bias before this proposed technique could ever be used.

The method propsed by the FP420 collaboration to determine the proton tagger positions at the LHC is to use the Beam Position Monitors (BPM), which are necessary in any case for monitoring the position of the extremely highmomentum proton beam. However, given that triggering on elastic $p \bar{p}$ collisions will be difficult (if not impossible) at 420 metres, a method similar to this that uses the data from the pots themselves to determine the pot positions may provide an alternative in the absence of the BPM. 


\section{Chapter 7}

\section{Search for Exclusive Di-Jets at DØ}

Having seen in section 4.6.3 that it is possible to test ExHuME, and the calculation upon which it is based, against di-jet production at the Tevatron, $p \bar{p} \rightarrow p+j j+\bar{p}$, this chapter describes attempts at $D \varnothing$ to isolate some exclusive di-jet events. The aim of this preliminary analysis was to select events with a di-jet system that contained a large fraction, $R_{j j}$, of the total mass available in the calorimeter. The distributions in figure 4.24 reveal that making a cut on the transverse momentum, $P_{\perp}$, of the leading jet isolates high $R_{j j}$ events in an exclusive sample, albeit at the expense of the available cross section. By measuring the proton longitudinal momentum losses, $x_{1,2}$, in the calorimeter rather than by using the proton taggers (the positions of which were not known for data taken at DØ, in any case) it was possible to apply a relatively high $P_{\perp}$ cut yet still probe the low $x_{1,2}$ region. Using the quadrupole spectrometers, on the other hand, would force a cut on $x_{1,2}$ because they do not have acceptance down to very low $x_{1,2}$.

The proton longitudinal momentum losses, $x_{1,2}$, were approximated by $x_{c a l_{1,2}}$ using the following summations over the calorimeter cells with rapidity $y_{i}$ and transverse momentum $P_{\perp_{i}}$ [76]:

$$
\begin{aligned}
& x_{c a l_{1}}=\sum_{\text {cells }} P_{\perp_{i}} e^{y_{i}} / \sqrt{s}, \\
& x_{c a l_{2}}=\sum_{\text {cells }} P_{\perp_{i}} e^{-y_{i}} / \sqrt{s} .
\end{aligned}
$$


Then the total invariant mass of the central system, $\sqrt{\hat{s}}$, is approximately $\sqrt{\hat{s_{c a l}}}$

$$
\sqrt{\hat{s_{c a l}}} \approx \sqrt{x_{c a l_{1}} x_{c a l_{2}} s}
$$

This analysis searched for events with $\sqrt{\hat{s}}<80 \mathrm{GeV}$ with at least one jet. The data used was taken during 2004 in the run range 188324 to 195839.

\subsection{Candidate Event Selection}

\subsubsection{Inclusive Background Simulated with Pomwig}

The main theoretical background to central exclusive production is inclusive dijet production through double Pomeron exchange or Reggeon exchange. The Pomeron and Reggeon emerge as trajectories in Regge theory [77]. In the limit that $s \ll t$ it can be shown [25] that the amplitude, $\mathcal{A}$ for a $2 \rightarrow 2$ scattering process relates to the collision energy $s$ as follows:

$$
\mathcal{A}(s, t) \propto s^{\alpha(t)}
$$

where $\alpha(t)$ is the location in the complex angular momentum plane of a pole in the partial wave amplitude at a given value of $t$. Partial wave amplitudes are used as the coefficients of the Legendre polynomials in an expansion of a cross section or amplitude in terms of its angular momentum states, or the contributions from those states. The total cross section is proportional to the forward $(t=0)$ scattering amplitude, so there are two possibilities: if $\alpha(0)<0$ then the total cross section will fall with increasing $s$ or if $\alpha(0)>0$ then the total cross section will rise with $s$. It is found that there are "trajectories" of $\alpha(t)$ that are linear in $t$ and provide different contributions to the cross section. The Pomeron trajectory has $\alpha(0)>0$, whereas reggeon trajectories have $\alpha(0)<0$. The pomeron trajectory corresponds to the exchange of a particle with the quantum numbers of the vacuum. In QCD, the existence of the pomeron is explained as a "ladder" of gluons (figure 7.1). However, the pomeron behaviour derived from a QCD ladder does not explain the softer $s$ dependence of the total $p p \rightarrow p p$ cross section. 


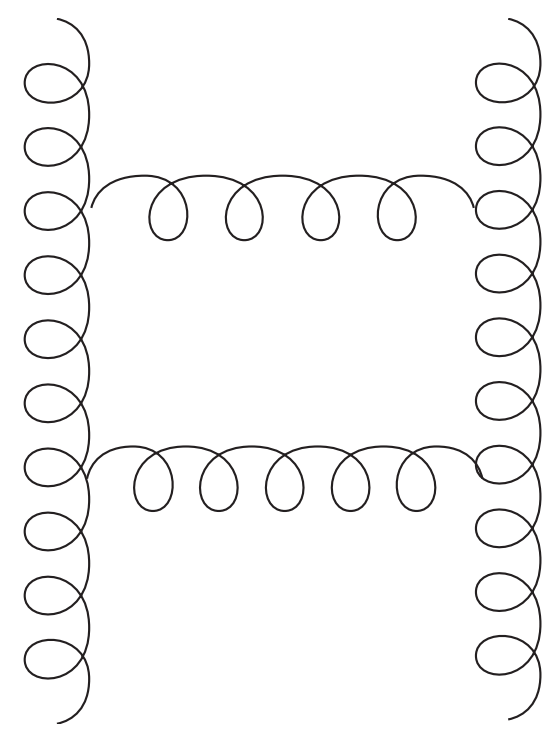

Figure 7.1: A ladder of gluons.

In double Pomeron exchange, shown in figure 7.2, each of the (anti)protons emits a colourless spin-zero Pomeron and the protons themselves remain intact, just as in central exclusive production. Gluons from each of the Pomerons fuse to form a di-jet system and the remnants of the Pomerons also produce jets, which are normally forward of the central jets. In the case that the Pomeron remnants are small or evade detection forward of the calorimeter's coverage, double Pomeron exchange has a very similar signature to central exclusive production. A challenge for studies of central exclusive production is therefore separating the double Pomeron from the central exclusive production.

Pomwig [78] is a Monte Carlo simulator of events involving single or double Pomeron exchange and has been tested against date from the Tevatron and HERA, see for example [22] for recent data from HERA. Pomwig was used here to provide a simulation of the double Pomeron exchange background.

\subsubsection{Triggering}

Events were selected that had the JT_15TT_GapSN trigger on, which requires two $3 \mathrm{GeV}$ calorimeter towers and both the south and north luminosity monitor to be off at Level 1 and a jet with at least $15 \mathrm{GeV}$ of transverse momentum at Level 3. 


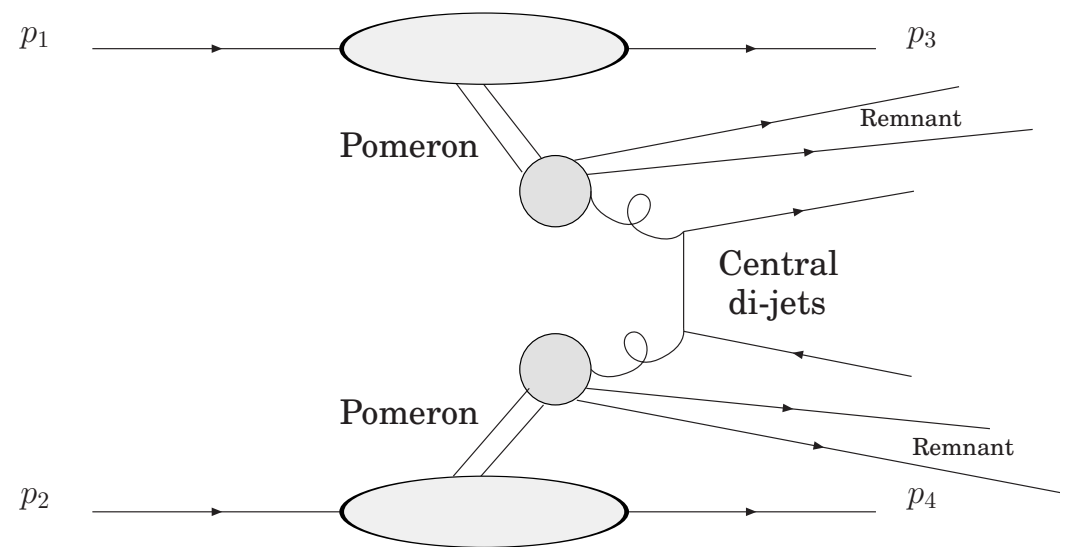

Figure 7.2: Production of a di-jet system through double Pomeron exchange showing the remnant jets from the Pomerons.

In a sample of jet events found with a trigger that is independent of the jet trigger, the jet trigger efficiency is defined as the fraction of events in which the jet trigger is active. The efficiency of the inclusive $15 \mathrm{GeV}$ jet trigger without the luminosity monitor requirement is shown in figure 7.3 and shows that the jet trigger turns on sharply at around $15 \mathrm{GeV}$ but is approximately flat for jets with $E_{T}>20 \mathrm{GeV}$. At jet transverse energies below $20 \mathrm{GeV}$ the trigger is biased towards higher transverse energies. There are therefore two reasons why the leading jet was required to have $E_{T}>20 \mathrm{GeV}$ : it ensures there is no bias due to the trigger and, as shown in figure 4.24 , it also favours high $R_{j j}$ events. The JT_15TT_GapSN trigger has a prescale in the range of 2 to 40 , however this does not affect the shape of kinematic distributions shown in this chapter.

\subsubsection{Calorimeter Calibration and Noise}

The energy that is deposited in a calorimeter cell is not identical with that reported by the readout electronics. Not only that, but each individual calorimeter cell has a different response to the same energy deposition. However, due to the symmetry of the calorimeter about the $\phi$ direction, the $\phi$ distribution of the energy deposited in the calorimeter over many bunch crossings should be isotropic. It is therefore possible to derive a set of calibration constants for the calorimeter cells that, when used to multiply the recorded cell energies, account for anisotropies 


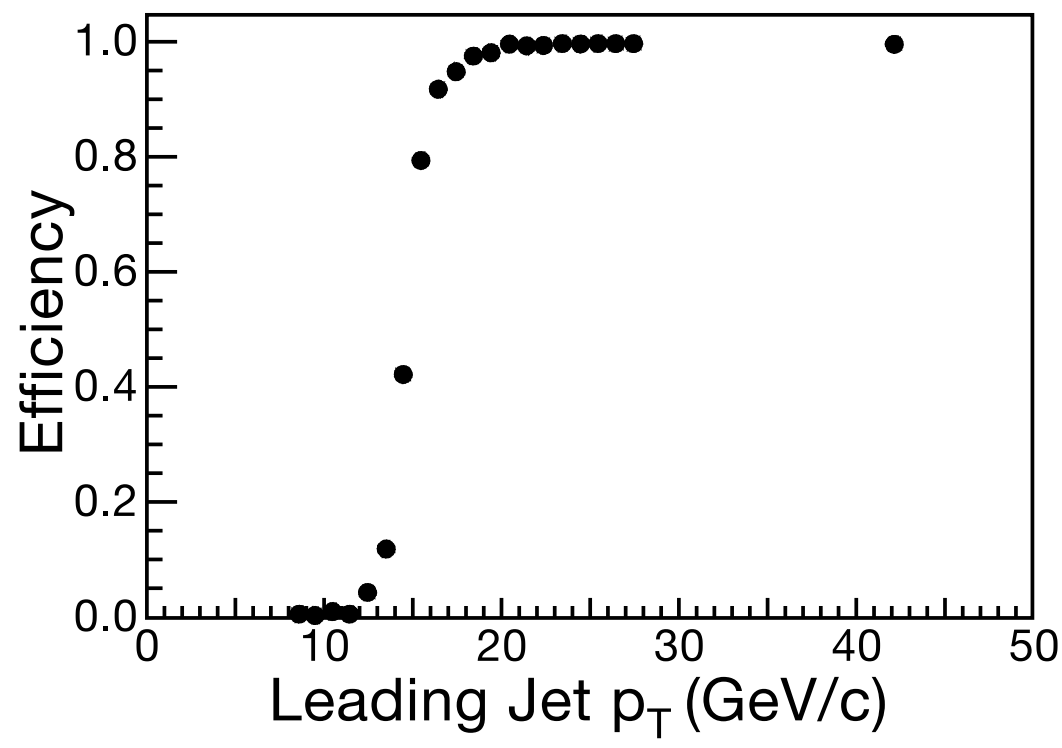

Figure 7.3: The Level 3 jet trigger efficiency taken from [64] showing the turn on at a jet transverse momentum $P_{\perp}$ of about $15 \mathrm{GeV}$.

in the calorimeter response. The calibration constant for the $i^{\text {th }}$ calorimeter cell may be approximated by $C_{i}$ as follows:

$$
C_{i} \simeq \frac{E_{t o t}}{N E_{i}}
$$

where $N$ is the number of calorimeter cells that lie within the same layer and at the same pseudo-rapidity as the $i^{t h}$ cell, $E_{t o t}$ is the total energy that is reported to be deposited in the $N$ cells over the course of many bunch crossings and $E_{i}$ is the total energy that is reported to be deposited in the $i^{\text {th }}$ cell over the same bunch crossings. Applying such a calibration constant to the calorimeter cell energies flattens the $\phi$ distribution of the energy deposited in the calorimeter.

A similar calibration can be applied in the longitudinal direction by using a sample of di-jet events. For a given jet $P_{T}$ and radius, the distribution of energy across the jet (known as the jet profile) should be the same for jets in two different parts of the calorimeter. Since a jet spans several cells with different pseudo-rapities, by forcing the jet profile to the same shape at all points in the calorimeter a callibration constant can be obtained.

Calorimeter calibration constants for calorimeter cells were provided in a 
file [79] for the central calorimeter $(|y|<1)$. The calorimeter cell energies were adjusted by multiplying by the calibration constant, where available.

Fluctuations in the grounding of the absorber in the calorimeter cells and cross talk between cables produces noise in the energy measurement of a cell; even when no radiation entered the cell it will still report a small $(\mathcal{O}(100 \mathrm{MeV}))$ energy deposition. Even a small amount of noise can make quite a large contribution to the $\hat{s}$ measurement if the event is boosted and the noisy cell is on the other side of the calorimeter to the boost direction. For example, if there is a low mass jet with three-momentum $\mathbf{p}_{\mathbf{j e t}}$ and a noisy cell that reports a small energy $E_{n}$ with momentum $-E_{n} \mathbf{p}_{\text {jet }} /\left|\mathbf{p}_{\text {jet }}\right|$ then the calculated $\hat{s}$ is approximately $\hat{s}_{\text {noise }}$ :

$$
\hat{s}_{\text {noise }} \simeq 2 \sqrt{E_{n}\left|p_{\text {jet }}\right|}
$$

A noise threshold is therefore required, below which cells are not included in jets or in the calculation of $\hat{s}$. Cell thresholds of $200 \mathrm{MeV}$ and $400 \mathrm{MeV}$ were used in the EM and FH layers of the calorimeter respectively [80]. In addition, the $\mathrm{CH}$ and ICD layers were discarded because of their high noise content. Ignoring the noisy $\mathrm{CH}$ and ICD layers should not affect the $R_{j j}$ measurement a great deal since the missing contribution to the di-jet mass cancels to some extent with the missing contribution to $\hat{s}$.

The "ring of fire" is a known problem with the DØ calorimeter in which all cells in a ring of the same pseudo-rapidity report a large $(\mathcal{O}(10 \mathrm{GeV}))$ energy deposition (figure 7.4). Ring of fire events are eliminated in double diffraction, however, because the $\hat{s}$ that is calculated from such a signal is too large to pass the diffractive cuts. Ring of fire events were also vetoed by discarding bad luminosity blocks (LBNS), which was done by removing all events for which the LBN was listed in [81].

\subsubsection{Jet Finder}

The active cells in the calorimeter were combined into towers of the same $\phi$ and $\eta$ and the pxcone cone jet algorithm, available as part of the hztool library [60], was run on the resulting set of four vectors. A jet radius of 0.7 with a maximum 


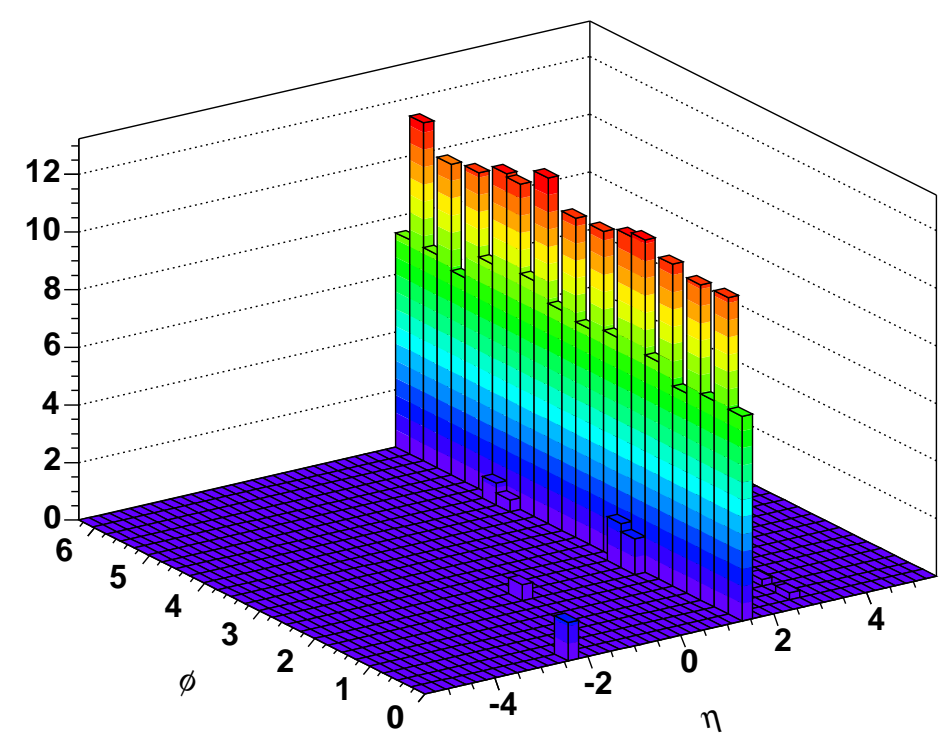

Figure 7.4: A "Ring of Fire" noise signal in the calorimeter.

overlap between jets of 0.5 was set. At least two jets were required to be identified in the calorimeter, and, as already explained in the introduction to this chapter and in section 7.1.2, the jet with the largest $P_{\perp}$ was required to have at least $20 \mathrm{GeV}$ of transverse momentum.

A jet energy scale (JES) correction would ideally be used to account for the loss of measured energy through holes and dead material in the calorimeter and to project the measured jet onto a particle level jet. However, the JES determined by [82] assumes that the event is non-diffractive and has no rapidity gap and hence includes the effect of both a soft underlying event and pile up on the jet energy. The JES correction is therefore not valid for diffraction $[83,84]$ and was not used. In any case, the JES will cancel in the numerator and denominator of $R_{j j}$ to some extent.

\subsubsection{Gap Cut and $x$ Correction}

A rapidity gap cut at $y=y_{c u t}$ requires that there be no activity in the calorimeter forward of $y_{\text {cut }}$ above that permitted by the noise threshold. Diffractive events are those for which $\hat{s} \ll s$ and, in principle, no $y_{c u t}$ need be specified in order to define a diffractive event. However, the calorimeter does not have unlimited 
rapidity coverage, so it is possible for radiation to evade detection in the forward regions, which results in an underestimation of the values for $x_{c a l_{1,2}}$. To ameliorate this situation, a gap cut was made so that any events with activity above the noise threshold towards the edge of the calorimeter were vetoed. If there is activity right at the edge of the calorimeter then it is likely that there is also activity outside of the calorimeter. Conversely, if there is no activity near the edge of the calorimeter then it becomes less likely that there is a significant amount of radiation beyond the calorimeter, other than the outgoing protons.

Requiring a rapidity gap also suppresses pile up and any soft underlying event (section 2.3), which should not exist for double pomeron exchange or central exclusive production. This is because any interactions in addition to the diffractive process of interest are likely to fill in the rapidity gap. This cut can pose a challenge when calculating the total integrated luminosity, which is necessary for the calculation of a cross section, however, since data taken at a high instantaneous luminosity is more likely to contain pile up and thus more events that are vetoed by the gap cut.

By running the Pomwig Monte Carlo, the generated proton momentum losses $x_{t r u_{1,2}}$ can be compared to $x_{c a l_{1,2}}$ (equation 7.1) and a table of the generated $x_{1,2}$ versus the $x_{c a l_{1,2}}$ reconstructed from final state particles emitted into the region of geometrical acceptance of the calorimeter can be produced. Pomwig was used to generate double diffractive di-jet events with an $x_{t r u_{1,2}}$ range of $0.01<$ $x_{1,2}<0.06$, which corresponds to an $\sqrt{\hat{s}}$ range of $20 \mathrm{GeV}<\sqrt{\hat{s}}<120 \mathrm{GeV}$. A minimum $P_{\perp}$ for the hard scatter of $P_{\perp}>14 \mathrm{GeV}$ was also set to improve the efficiency of the Pomwig generation without affecting the results.

The three-vectors of the final state hadrons output by Pomwig were adjusted to lie along the nearest calorimeter tower and any particle with less than $1 \mathrm{GeV}$ of energy or lying outside of the geometrical acceptance of the calorimeter was discarded. Adjusting the three-vectors and keeping the particle on shell resulted in a corresponding adjustment of the particle's energy and smeared the energy distribution of the particles. The jet finder was run on the resulting system of particles and events were selected with the lead jet $P_{\perp}>20 \mathrm{GeV}$. For each event 


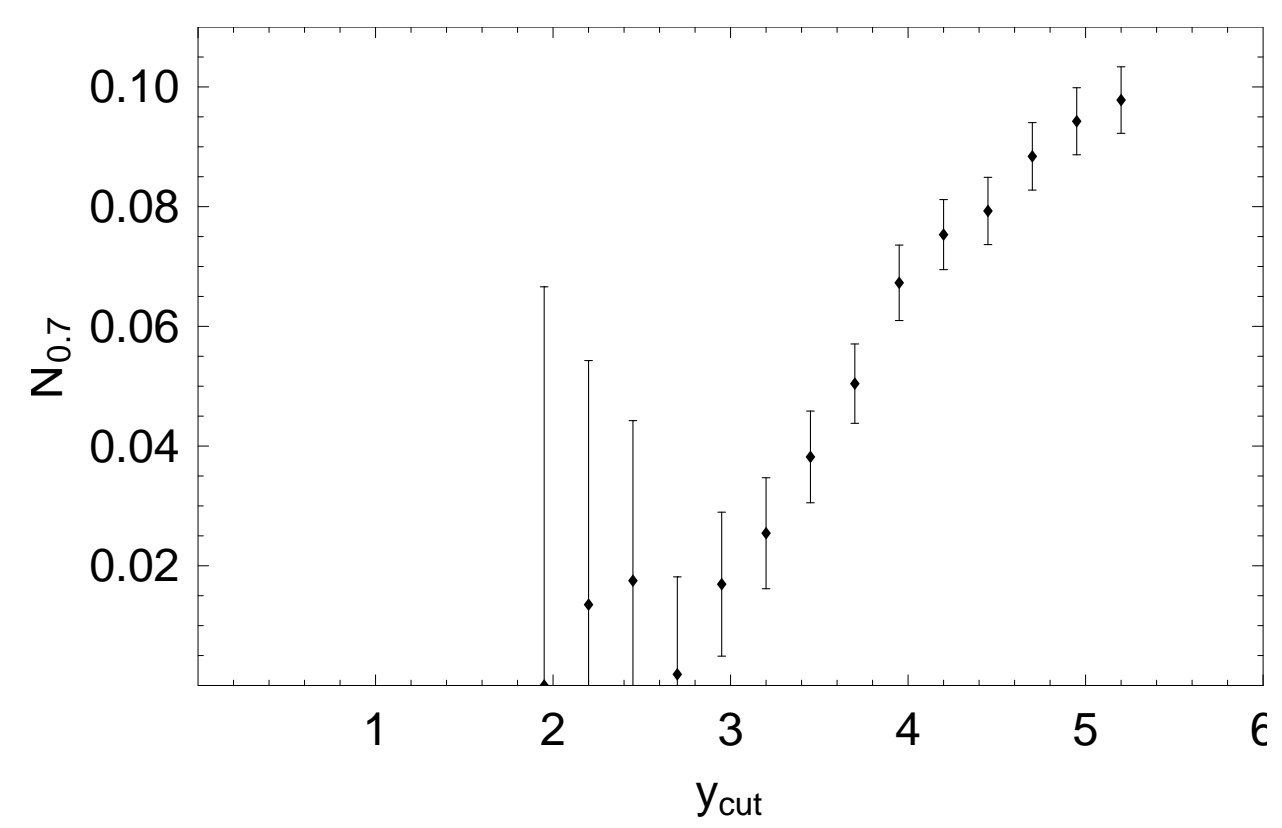

Figure 7.5: The fraction of events with $x_{c a l} / x_{t r u}<0.7$ versus the rapidity gap cut that is made.

the ratio $x_{c a l} / x_{t r u}$ was calculated.

In order to determine an optimum value for $y_{c u t}$ a succession of rapidity gap cuts in the range $2.0<y_{c u t}<5.5$ were made on the Pomwig events and the fraction of events that had $x_{c a l} / x_{t r u}<0.7$ was calculated in each case, as shown in figure 7.5. A larger rapidity gap requirement results in more reliable estimates for $x_{1,2}$ by rejecting those events that are likely to have a poor value for $x_{c a l} / x_{t r u}$. The improvement in the estimate for $x_{1,2}$ comes at the expense of the total number of events left after the cut is made. Based on figure 7.5, the gap cut in this analysis was made at $y_{c u t}=3.2$ since a larger gap cut (i.e. lower $y_{c u t}$ ) does not significantly improve the $x_{1,2}$ reconstruction.

Scatter plots of correction factor $x_{c a l} / x_{t r u}$ versus $x_{c a l}$ with $y_{c u t}=3.2$ are shown in figure 7.6, together with the average and $1 \sigma$ band for the distributions of points. It is possible to use figure 7.6 to correct the $x_{c a l_{1,2}}$ reconstructed from data back to the true $x_{1,2}$, in which case the $1 \sigma$ band, which is approximately 0.2 wide, leads to a significant uncertainty of around $20 \%$ on the measurement of the $x_{1,2}$. Note that this uncertainty disappears if forward proton taggers are used to measure $x_{1,2}$ directly, which is a key requirement for making an accurate 

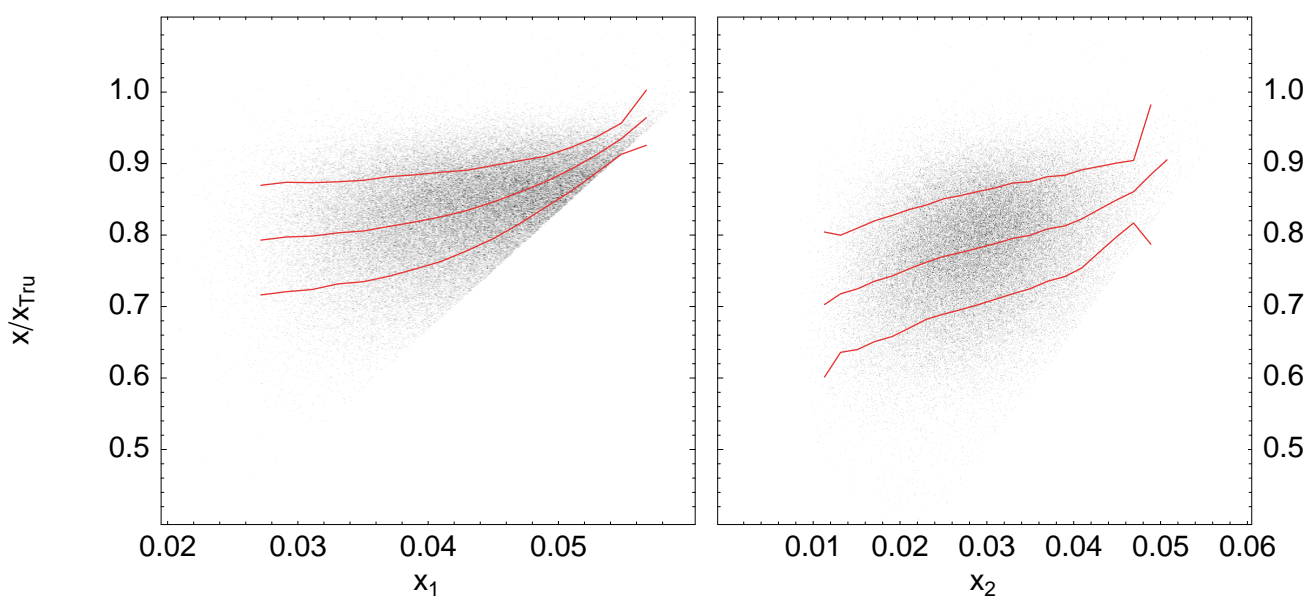

Figure 7.6: A scatter plot of the ratio $x_{c a l} / x_{t r u}$ versus $x_{c a l}$ from 100,000 Pomwig events for the largest (left) and smallest (right) in the event. A gap cut of 3.2 was made on both sides of the calorimeter and the largest jet was required to have a $P_{T}>20 \mathrm{GeV}$. The average correction is shown as a line together with the $1 \sigma$ band for the distribution.

measurement of the central exclusive production cross section. In any case, this preliminary analysis uses uncorrected data points. Figure 7.6 shows a rise in the average value of $x_{c a l} / x_{t r u}$ with increasing $x_{c a l}$ and in the left hand panel there is a diagonal boundary, to the bottom right of which lie no points in the scatter plot. This is a result of the Monte Carlo being run with $x_{t r u}<0.06$, so as $x_{c a l} \rightarrow 0.06$ $x_{c a l} / x_{t r u} \rightarrow 1$.

\subsubsection{Kinematic Cuts}

A cut was made on the values of $x_{1}$ and $x_{2}$ such that

$$
x_{1,2}<0.04
$$

which corresponds to an upper limit on the total invariant mass in the calorimeter of approximately $80 \mathrm{GeV}$. Were $x$ corrections to be made a central mass of $80 \mathrm{GeV}$ would be expected to give a true central mass of approximately $100 \mathrm{GeV}$. These cuts on $x_{1,2}$ therefore satisfy the expectation for diffractive events that $1 \gg \sqrt{\hat{s} / s} \simeq 0.05$ This $x_{\text {cal }}$ distributions for the selected events are shown compared to Monte Carlo (see next section) in figure 7.16 
Since $\left|t_{1,2}\right| \lesssim 1 \mathrm{GeV}^{2}$, the vectorial sum of the total $P_{\perp}$ in the calorimeter should be less than $2 \mathrm{GeV}$. However, calorimeter noise makes such a cut too tight, so a cut on total $P_{T}$ in the calorimeter of $10 \mathrm{GeV}$ was made.

\subsection{Monte Carlo Comparison}

Along with 9.5 million Pomwig di-jet events, 10 million di-jet events were generated in ExHuME in the central mass range $29 \mathrm{GeV}<\sqrt{\hat{s}}<120 \mathrm{GeV}$. The strategy employed to ensure exactly the same analysis was applied to the Monte Carlo samples as to the data was to format the Monte Carlo samples so that they were in an identical format to the data and the same analysis program could be run on both. In order to do this, the particle four-vectors from Monte Carlo were adjusted slightly so that they aligned with the nearest calorimeter cell. This also resulted in a smearing of the Monte Carlo particle $E_{T}$ s. Any Monte Carlo particle that lay outside of the geometrical acceptance of the calorimeter was also discarded.

The cross sections for the Pomwig and ExHuME samples before and after analysis (summarised in section 7.4) are shown in table 7.1. Note that the Pomwig cross sections given in table 7.1 do not include a soft survival factor, whereas ExHuME uses a soft survival factor of 0.045 at a centre of mass energy of $2 \mathrm{TeV}$. The soft survival is an unknown parameter and, although there is no known reason why the soft survival should be vastly different between ExHuME and Pomwig, a soft survival for Pomwig of $\mathcal{S}=0.1$ was explored, along with $\mathcal{S}=0.045$

\subsection{Threshold for Particle Detection}

One of the effects that could cause non-exclusive di-jet events to appear to be exclusive at $D \varnothing$ would be if the calorimeter systematically failed to detect soft radiation below a certain threshold. The missed soft radiation would then not be present in the calculation of $\hat{s}$ and the value of $R_{j j}$ would appear larger than it should. Clearly, there is some threshold below which radiation cannot be 


\begin{tabular}{|c|c|c|c|c|c|c|}
\hline & \multicolumn{2}{|c|}{ Before Analysis } & \multicolumn{4}{c|}{ After Analysis } \\
\hline & Events (Generated) & Cross Section $[\mathrm{pb}]$ & \multicolumn{2}{c|}{ Events (passed cuts) } & \multicolumn{2}{c|}{ Cross Section [pb] } \\
\hline Threshold ${ }_{\text {[Gev] }}$ & & & 1 & 2 & 1 & 2 \\
\hline Pomwig & $9.5 \times 10^{6}$ & $5 \times 10^{4}$ & 28121 & 23266 & 187000 & 155000 \\
\hline ExHuME & $10^{7}$ & $2 \times 10^{4}$ & 10881 & 6199 & 21800 & 12400 \\
\hline
\end{tabular}

Table 7.1: Monte Carlo cross sections and number of events before and after analysis. The ExHuME cross sections include a soft survival, $\mathcal{S}$, of $\mathcal{S}=0.045$, whereas the Pomwig values do not.

detected. At the very least, the $200 \mathrm{MeV}$ cut on cell energy to reduce noise implies that no particle with less than $200 \mathrm{MeV}$ may be observed. The true threshold below which particles cannot be observed may be much higher.

The question then arises: how big does the threshold have to be before nonexclusive (Pomwig) di-jets appear to be exclusive? To attempt to answer this, the Monte Carlo samples were adjusted by discarding all particles with energy $E_{i}$ for which $E_{i}<E_{\text {Thresh. }}$. Four different values for $E_{\text {Thresh }}$ of $0.5,1,2$, and $4 \mathrm{GeV}$ were applied to give four Monte Carlo samples for comparison with data.

\subsection{Summary of Analysis cuts}

The selection criteria and cuts applied to both data and Monte Carlo samples are summarised here:

- Require JT_15TT_GapSN trigger to have fired (only applies to data).

- Require that the event is not present in the list of bad luminosity blocks.

- Remove from the data all EM calorimeter cells with less than $200 \mathrm{MeV}$ of energy and EM calorimeter cells with less than $400 \mathrm{MeV}$. Equivalently, remove from the Monte Carlo sample all particles with energy less than $0.5,1,2$ or $4 \mathrm{GeV}$.

- Require no radiation forward of $y=3.2$. 
- Require $x_{\mathrm{cal}_{1}} x_{\mathrm{cal}_{2}}<0.0016$ (equation 7.1), corresponding to approximately $\sqrt{\hat{s_{\text {cal }}}}<80 \mathrm{GeV}$.

- Require $x_{c a l_{1}}<0.04$ and $x_{c a l_{2}}<0.04$.

- Run the cone algorithm with a radius of 0.7 and an overlap parameter of 0.5 on the calorimeter towers. Require at least two jets and the highest $E_{T}$ jet must have $E_{T}>20 \mathrm{GeV}$.

- Require the calorimeter to have missing $E_{T}$ no greater than $10 \mathrm{GeV}$.

\subsection{Results}

Out of the 300 runs processed 126 events passed all cuts. The distribution of the angle $\phi_{j j}$ between the two leading jets in each event (figure 7.7) verifies that the 126 candidate events contain a back-to-back di-jet system. Unless otherwise stated, all curves shown in this section are normalised to unit area. Since the data is not corrected for acceptance and other detector effects, the error bars indicate statistical uncertainty only.

Figure 7.8 shows the $R_{j j}$ distribution for the data compared to Pomwig with particle thresholds of $0.5,1,2$ and $4 \mathrm{GeV}$. The Pomwig prediction only fits the data in the $4 \mathrm{GeV}$ case. An important issue for this analysis is therefore to determine whether the 200 and $400 \mathrm{MeV}$ noise cuts on the calorimeter cell energies could possibly imply that $\mathrm{D} \varnothing$ would fail to detect a particle with less that $4 \mathrm{GeV}$ of energy. Although a properly functioning calorimeter should not fail to observe a particle with close to $4 \mathrm{GeV}$, such a threshold would allow Pomwig to describe the data and must be completely ruled out in order to conclusively show central exclusive production.

To this end, the jet finder with a cone radius of $R=0.015$ was run on both the Monte Carlo sample and the calorimeter cells in the data. Such a cone radius is slightly larger than the $\mathrm{D} \varnothing$ calorimeter cell size and should correspond to one calorimeter tower per jet. Figure 7.9 shows the energy spectrum of the $R=0.015$ jets for data and Pomwig with thresholds of $0.5,1$ and $4 \mathrm{GeV}$ on the Monte Carlo 
particle energy. The lowest energy bins for each of the curves in figure 7.9 have been aligned with each other because they all correspond to the lowest energy tower that is detectable, whatever that lower threshold may be. The important qualitative feature of figure 7.9 is that the distribution of tower energies from Pomwig is too flat if a threshold of $4 \mathrm{GeV}$ is used.

The total number of jets, using a jet radius of 0.7, is shown in figure 7.10. Both Pomwig and ExHuME predict the correct relative numbers of events if a particle threshold of $1 \mathrm{GeV}$ is used. If a threshold of $4 \mathrm{GeV}$ is used then further particles are removed from each event and some of the three-jet events become two-jet events. The Pomwig curve with a $4 \mathrm{GeV}$ threshold does not fit the data as well as the curve with a $1 \mathrm{GeV}$ threshold.

The total number of $R=0.015$ jets in each event, $N_{P}$ is shown in figure 7.11. Neither a threshold of 1 or $4 \mathrm{GeV}$ fits the data. If a threshold of $4 \mathrm{GeV}$ is used then Pomwig predicts there are no events with greater than $10 R=0.015$ jets, whereas the data show events with over 50 such jets. Taken together, figures 7.9-7.11 suggest that Pomwig with around a $1 \mathrm{GeV}$ threshold is a better description of the data than a $4 \mathrm{GeV}$ threshold.

Figures 7.12 to 7.14 show the $R_{j j}$ distributions in data compared to Monte Carlo with different soft survival factors and particle thresholds. Figure 7.12, with equal soft survival and a threshold of $1 \mathrm{GeV}$ provides the best fit to the data. Figure 7.14, with a threshold of $2 \mathrm{GeV}$ predicts an extremely large spike at $R_{j j}=1$, although even in this case, Pomwig alone is insufficient to describe the data.

Figure 7.15 show the rapidity distribution of the central system, with the Monte Carlo predictions normalised so that they are aligned with the data points at $y=0$. Both Pomwig and ExHuME with a threshold of $1 \mathrm{GeV}$ provide a reasonable fit to the data, although the combined plot with equal soft survival factors is slightly better than either of the individual Monte Carlo curves.

Despite the similar rapidity distributions for ExHuME and Pomwig, the $x_{1,2}$ distributions are quite different because central exclusive production favours a lower central mass compared to double Pomeron exchange. This is shown 
in figure 7.16 where the observed $x_{\text {cal }}$ are plotted together with the Pomwig and ExHuME predictions. Pomwig predicts that the maximum $x_{c a l}$ value, $x_{1}$ continues to rise past 0.04, whereas the data show that it flattens off, which ExHuME predicts. Similarly, ExHuME agrees with the peak in the minimum $x$ value of about 0.02 , whereas Pomwig predicts a peak at a somewhat higher value of around 0.03 .

Events were selected with $R_{j j}>0.85$, which is slightly to the right of the crossing points of the ExHuME and Pomwig curves in figure 7.12. For these events, of which there were 64 in total, the number in which the leading jet had $E_{T}>E_{T}^{\prime}$ were counted for successive values of $E_{T}^{\prime}$ from 20 to $35 \mathrm{GeV}$, effectively giving the quantity $\sigma\left(E_{T}>E_{T}^{\prime}\right) / \sigma\left(R_{j j}>0.85\right)$ :

$$
\frac{\sigma\left(E_{T}>E_{T}^{\prime}\right)}{\sigma\left(R_{j j}>0.85\right)}=\frac{1}{N} \int_{E_{T}^{\prime}}^{\infty} \frac{\partial N}{\partial E_{T}} d E_{T}
$$

The quantity in equation 7.7 is shown in figure 7.17 for data, Pomwig and ExHuME with particle thresholds of $1 \mathrm{GeV}$. In each case the first point has been set to unity on the vertical axis. Pomwig and ExHuME are indistinguishable in figure 7.17 and both provide a good fit to the data. The jet $E_{T}$ behaviour of the so-called Saclay model, which is implemented in the DPEMC Monte Carlo simulator [59], is expected to differ from that of the calculation implemented in ExHuME. Quantities such as that in equation 7.7, as plotted in figure 7.17 should show significant differences between ExHuME and DPEMC.

Finally, figure 7.18 shows the location of calorimeter activity above the noise thresholds in the $\eta$ - $\phi$ plane. The top diagram shows an event with moderate $R_{j j}$ of $R_{j j}=0.68$, the region dominated by double pomeron exchange (Pomwig), whilst the bottom diagram shows the event with the largest $R_{j j}$ found in the data set, which is indistinguishable from $R_{j j}=1$. 


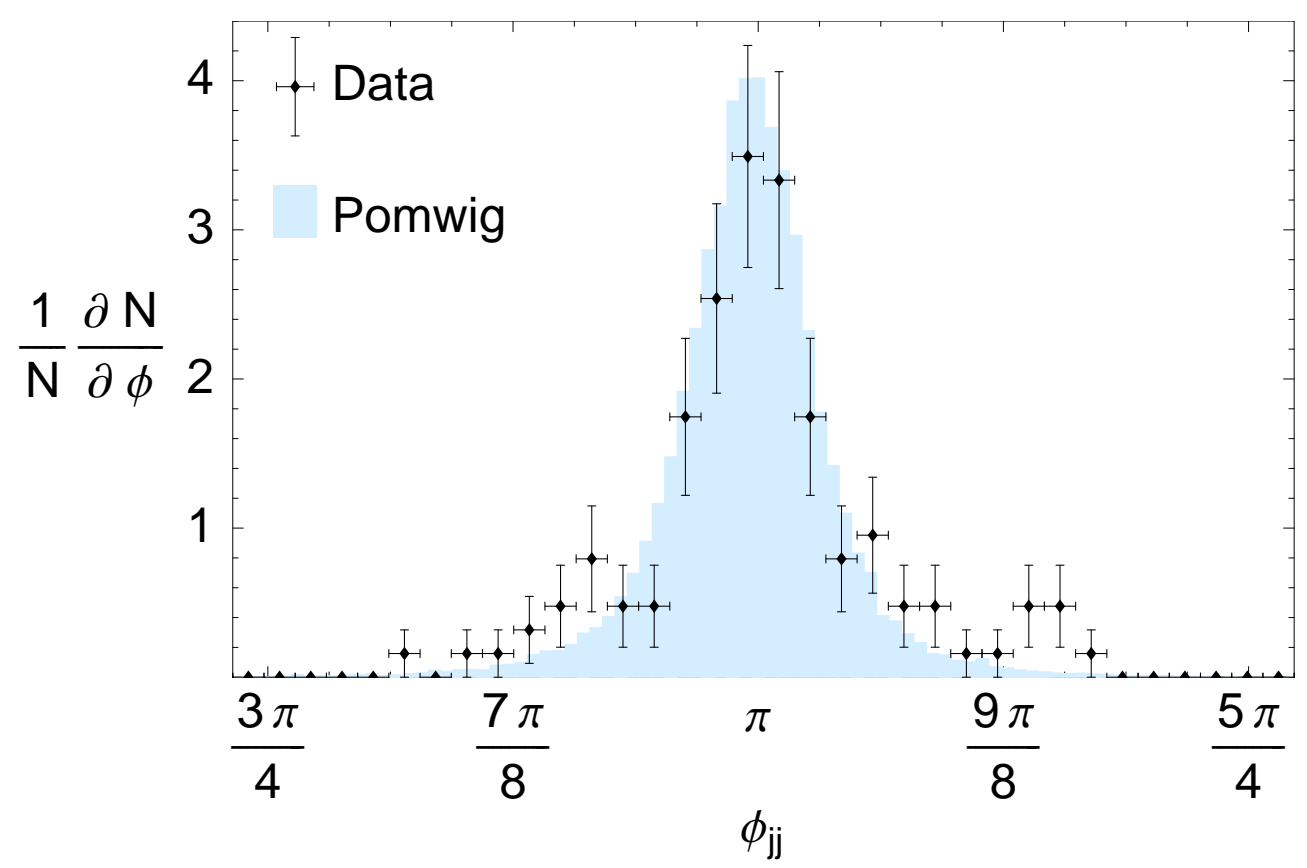

Figure 7.7: The angle $\phi_{j j}$ between the two leading jets in data and Pomwig

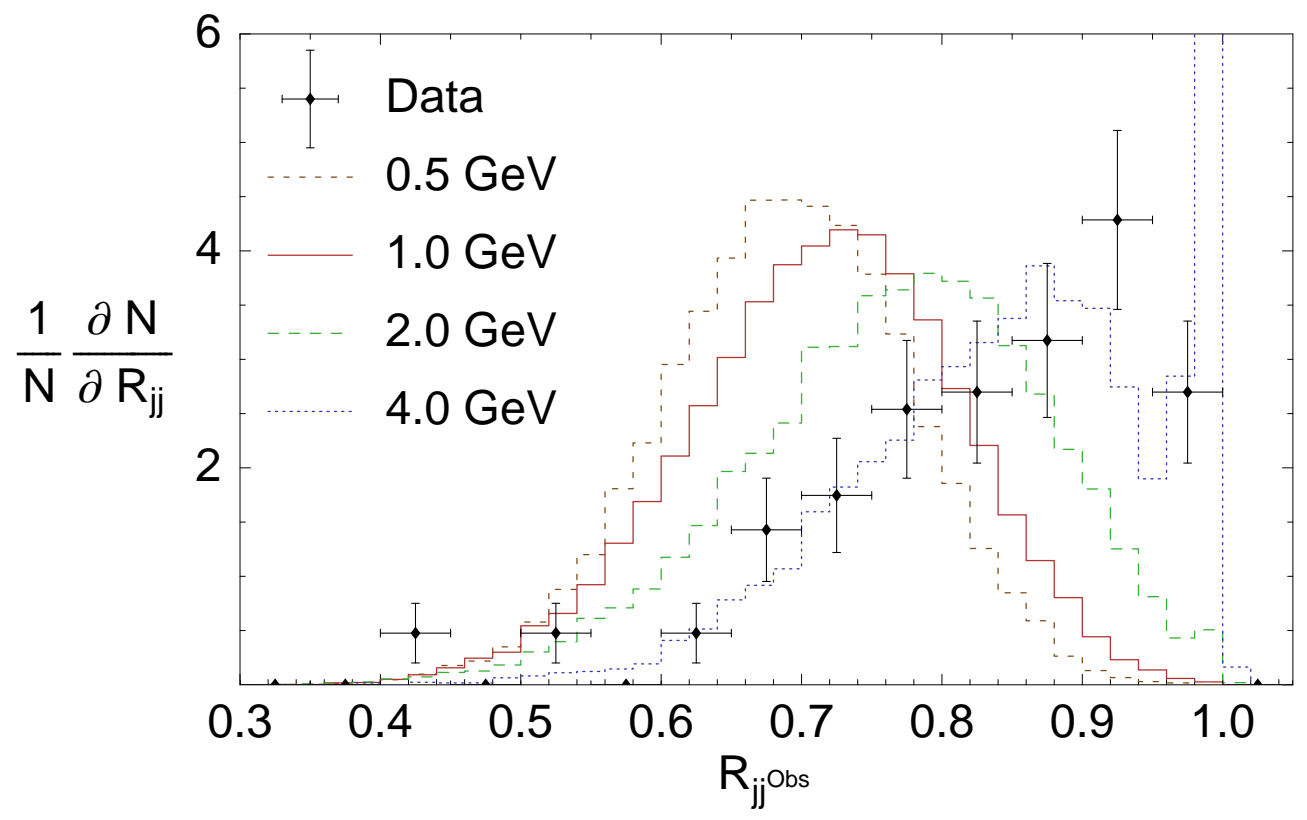

Figure 7.8: The observed $R_{j j}$ distribution and the $R_{j j}$ calculated in events generated by Pomwig with particle thresholds of $0.5,1,2$, and $4 \mathrm{GeV}$ 


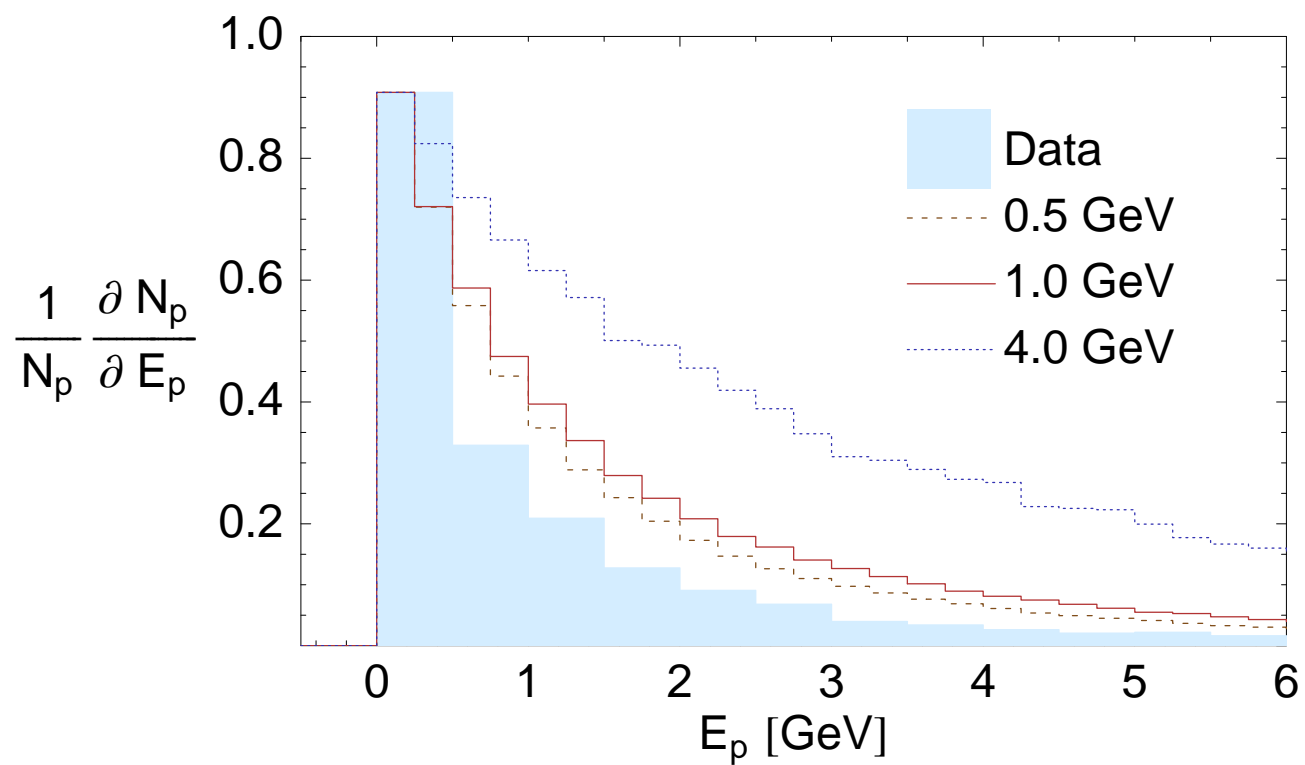

Figure 7.9: The observed tower energy spectrum, $E_{p}$, and the $E_{p}$ distributions from Pomwig with particle energy thresholds of 0.5, 1 and $4 \mathrm{GeV}$. The Pomwig curves have been shifted to the left by the threshold values in order that they may line up with the data.

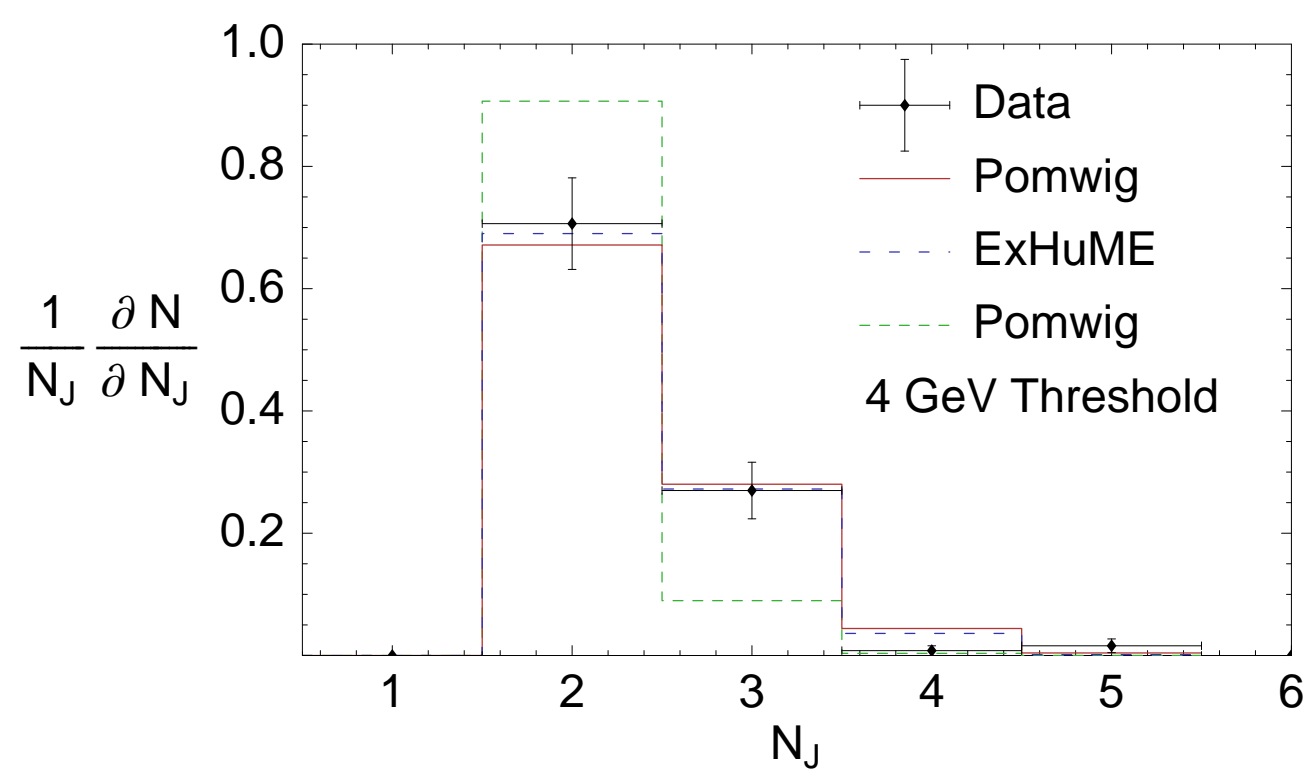

Figure 7.10: The Number of $R=0.7$ jets, $N_{J}$, in each event in data, Pomwig and ExHuME with $1 \mathrm{GeV}$ particle thresholds. The number of jets is also shown for Pomwig with a $4 \mathrm{GeV}$ particle threshold. 


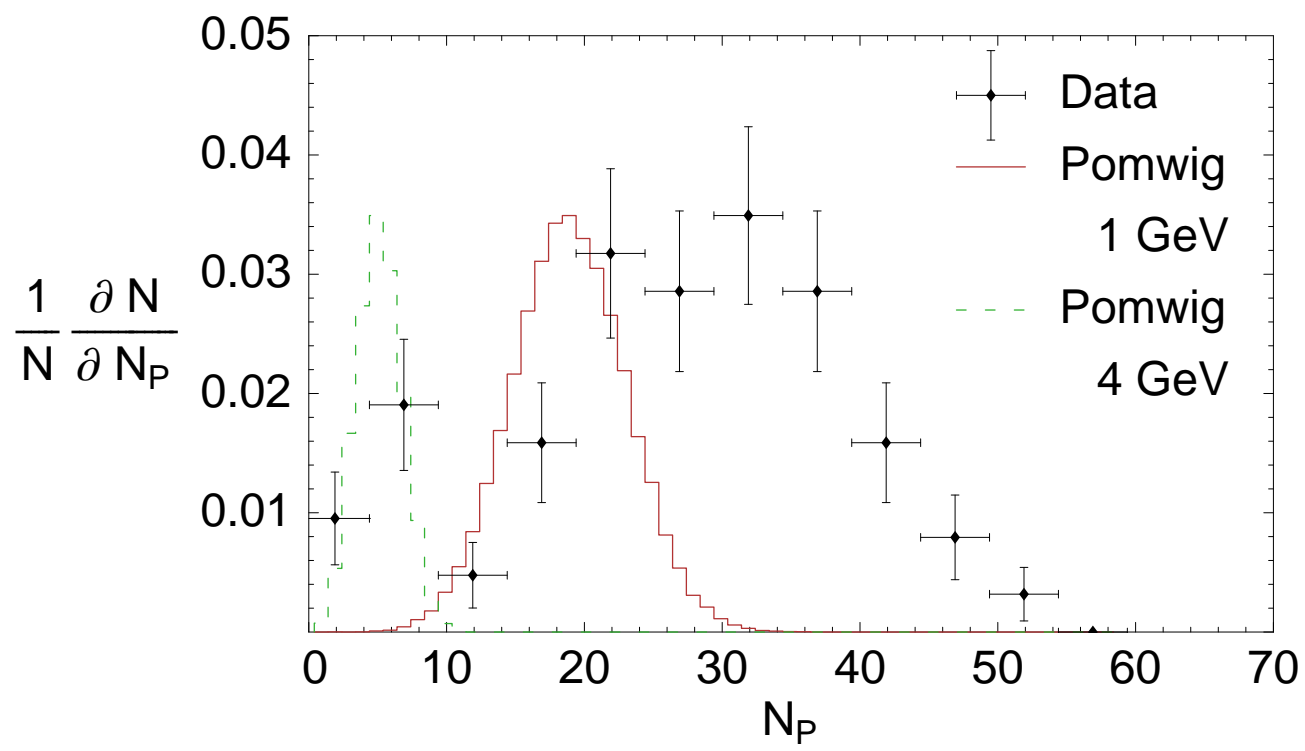

Figure 7.11: The $R=0.015$ jet multiplicity, $N_{p}$, in data and from Pomwig with particle thresholds of 1 and $4 \mathrm{GeV}$.

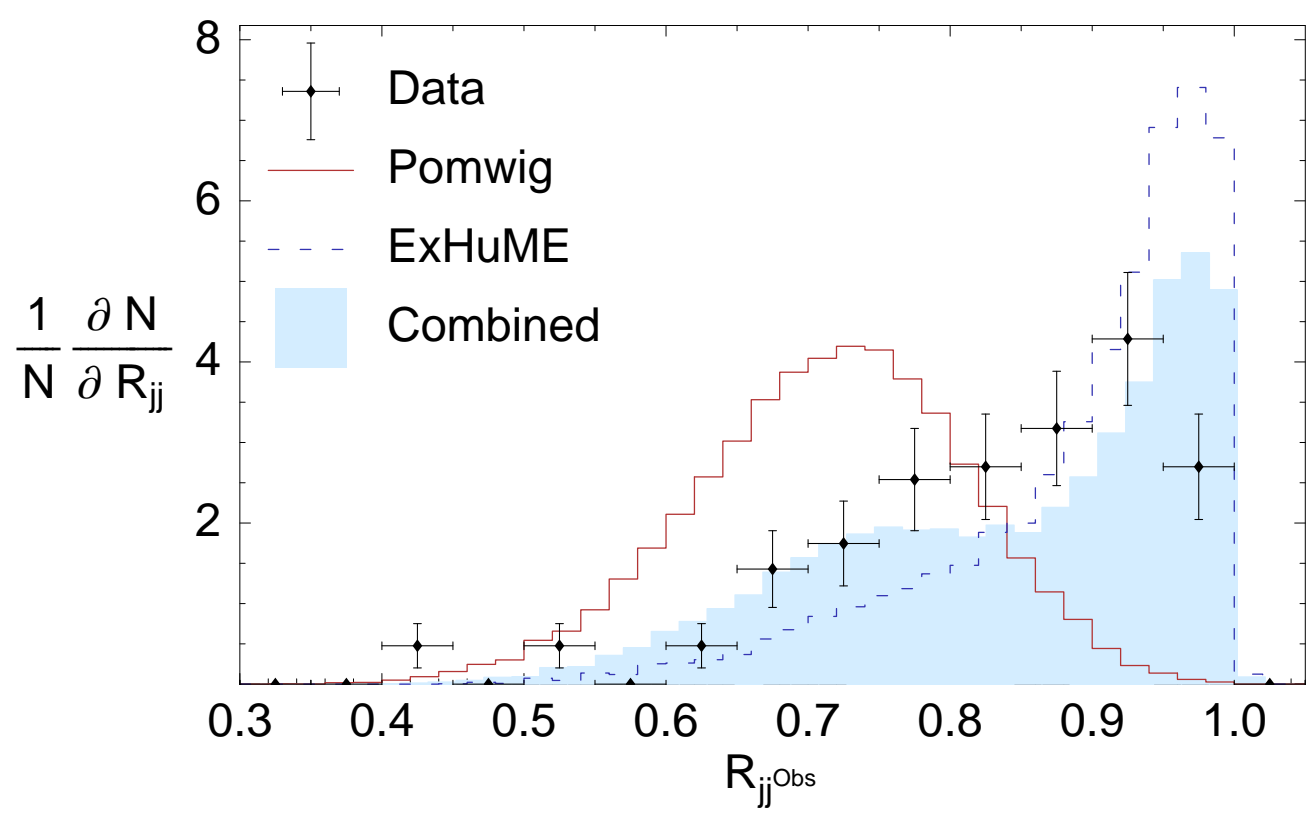

Figure 7.12: The di-jet mass fraction $R_{j j}$. The Monte Carlo plots have a threshold on particle energy of $1 \mathrm{GeV}$ and Pomwig and ExHuME have equal soft survival factors. 


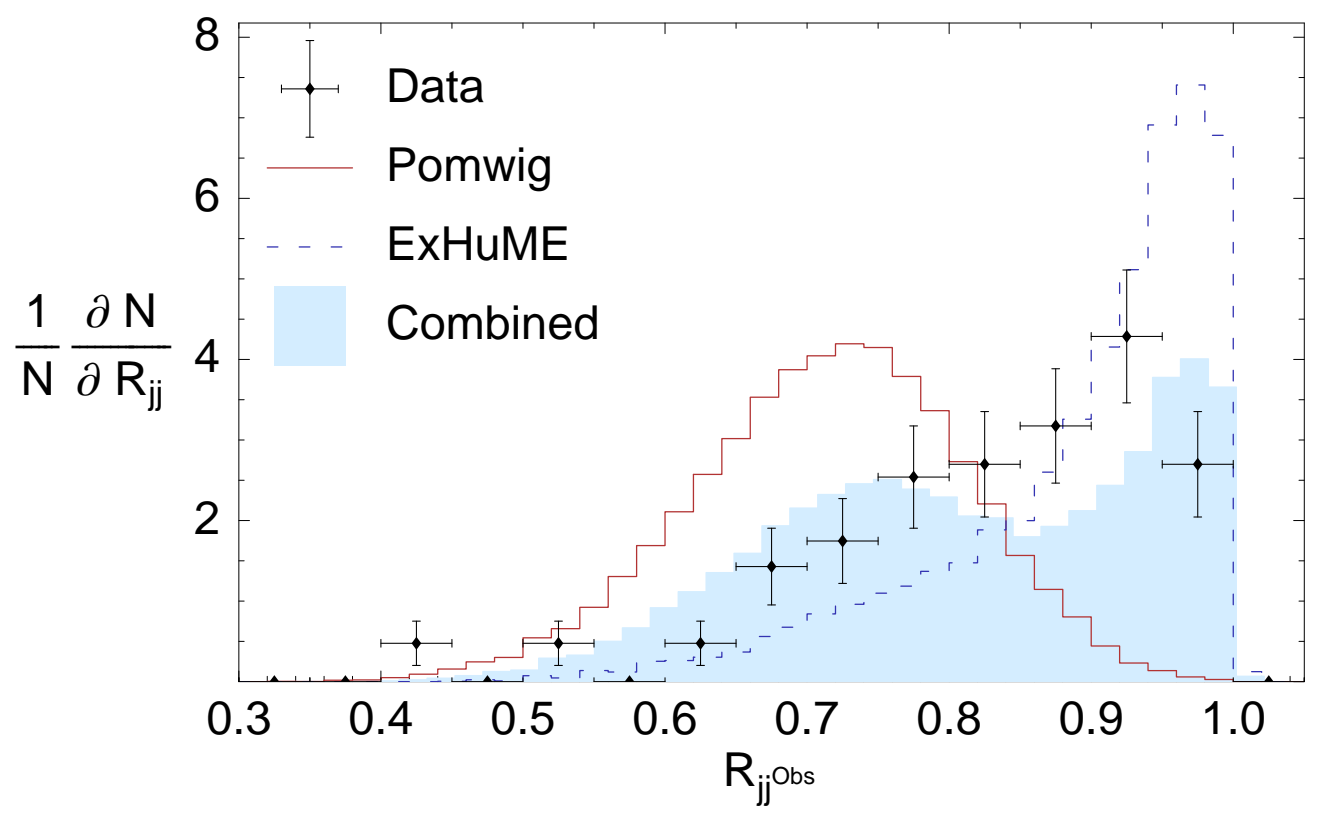

Figure 7.13: The di-jet mass fraction $R_{j j}$. The Monte Carlo plots have a threshold on particle energy of $1 \mathrm{GeV}$. Pomwig and ExHuME have soft survival factors of $\mathcal{S}=0.1$ and $\mathcal{S}=0.045$ respectively.

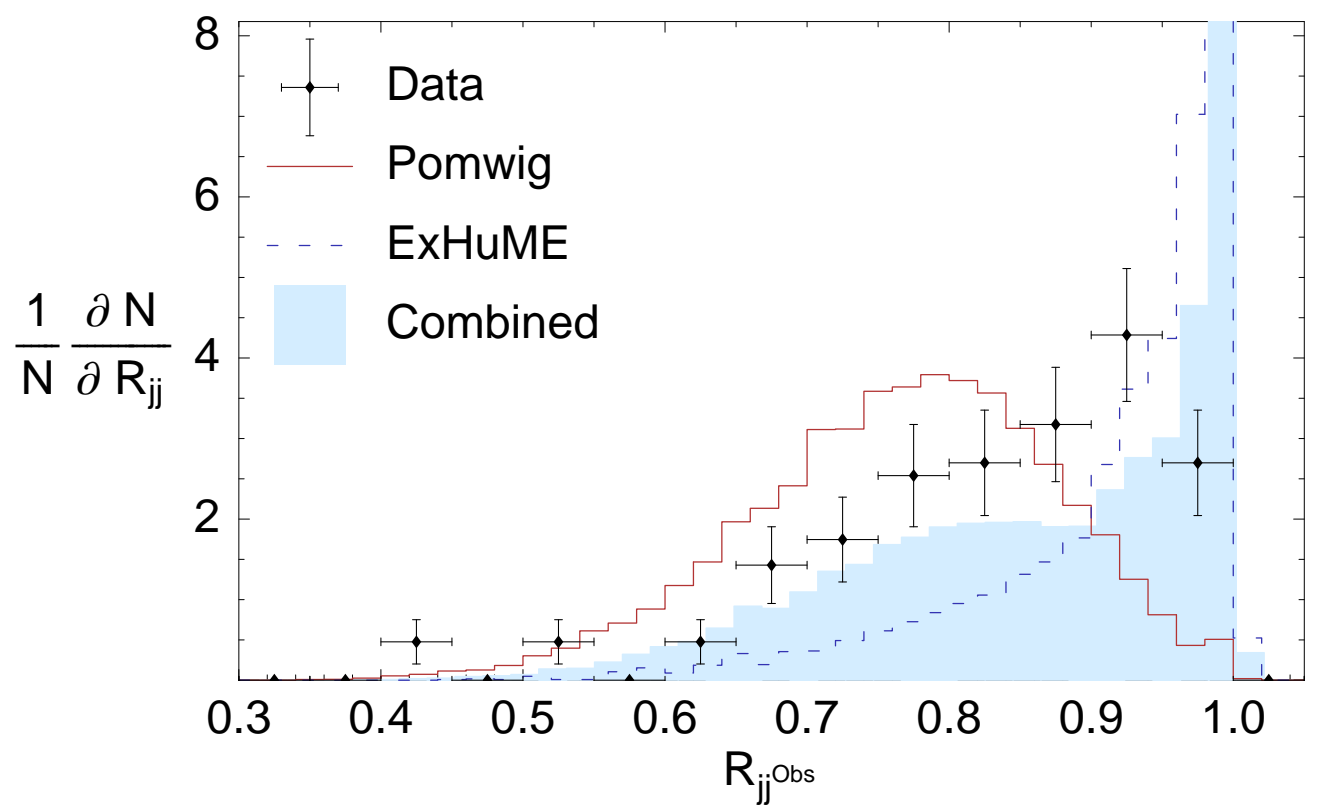

Figure 7.14: The di-jet mass fraction $R_{j j}$. The Monte Carlo plots have a threshold on particle energy of $2 \mathrm{GeV}$ and Pomwig and ExHuME have equal soft survival factors. 


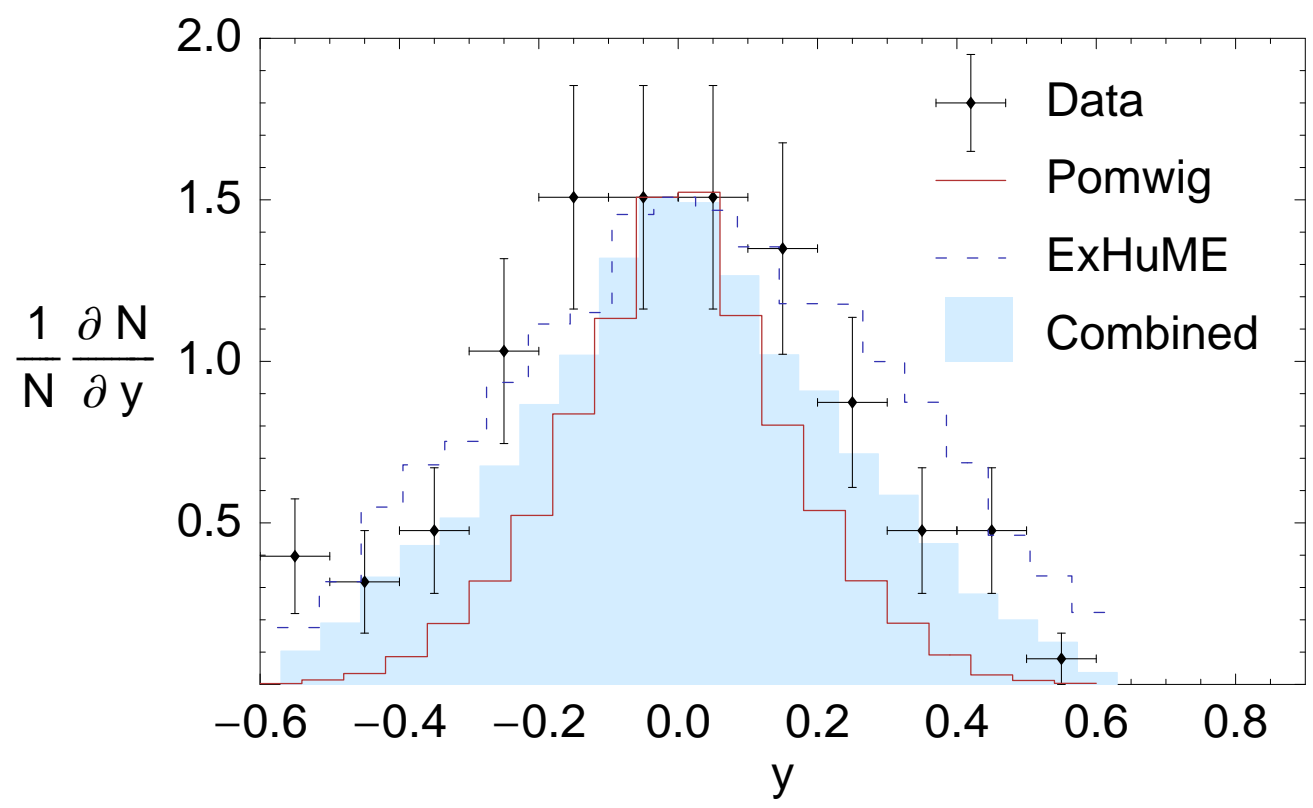

Figure 7.15: The rapidity distribution of the central system in data, Pomwig and ExHuME.
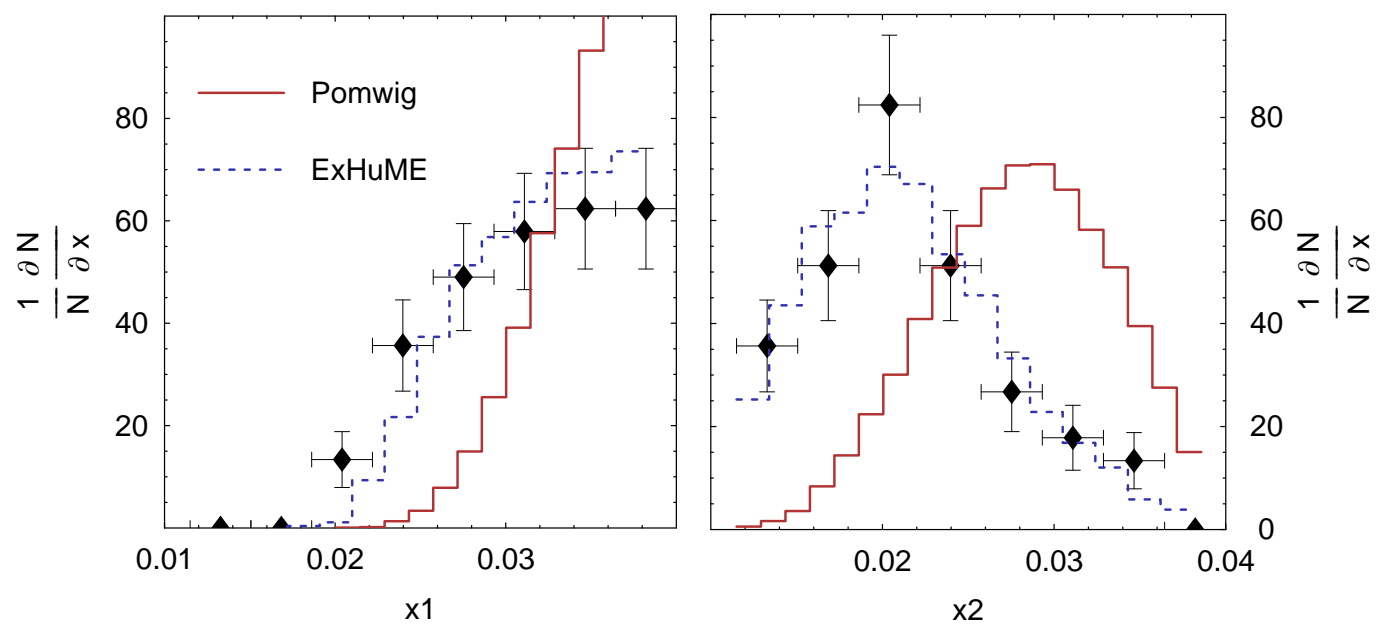

Figure 7.16: The maximum (left) and minimum (right) $x_{c a l}$ distributions in data, Pomwig and ExHuME. 


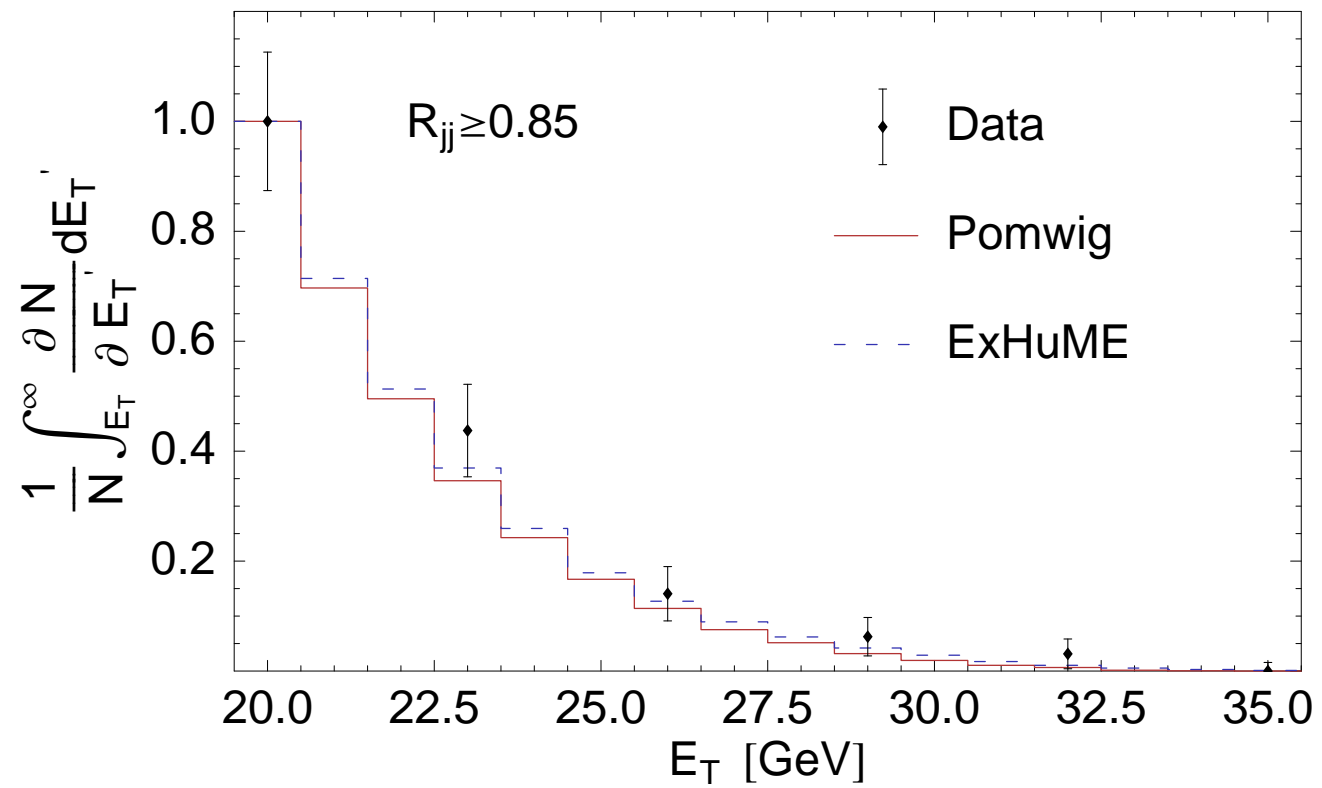

Figure 7.17: The number of events in which the leading jet had transverse energy above $E_{T}$ and $R_{j j}>0.85$ 

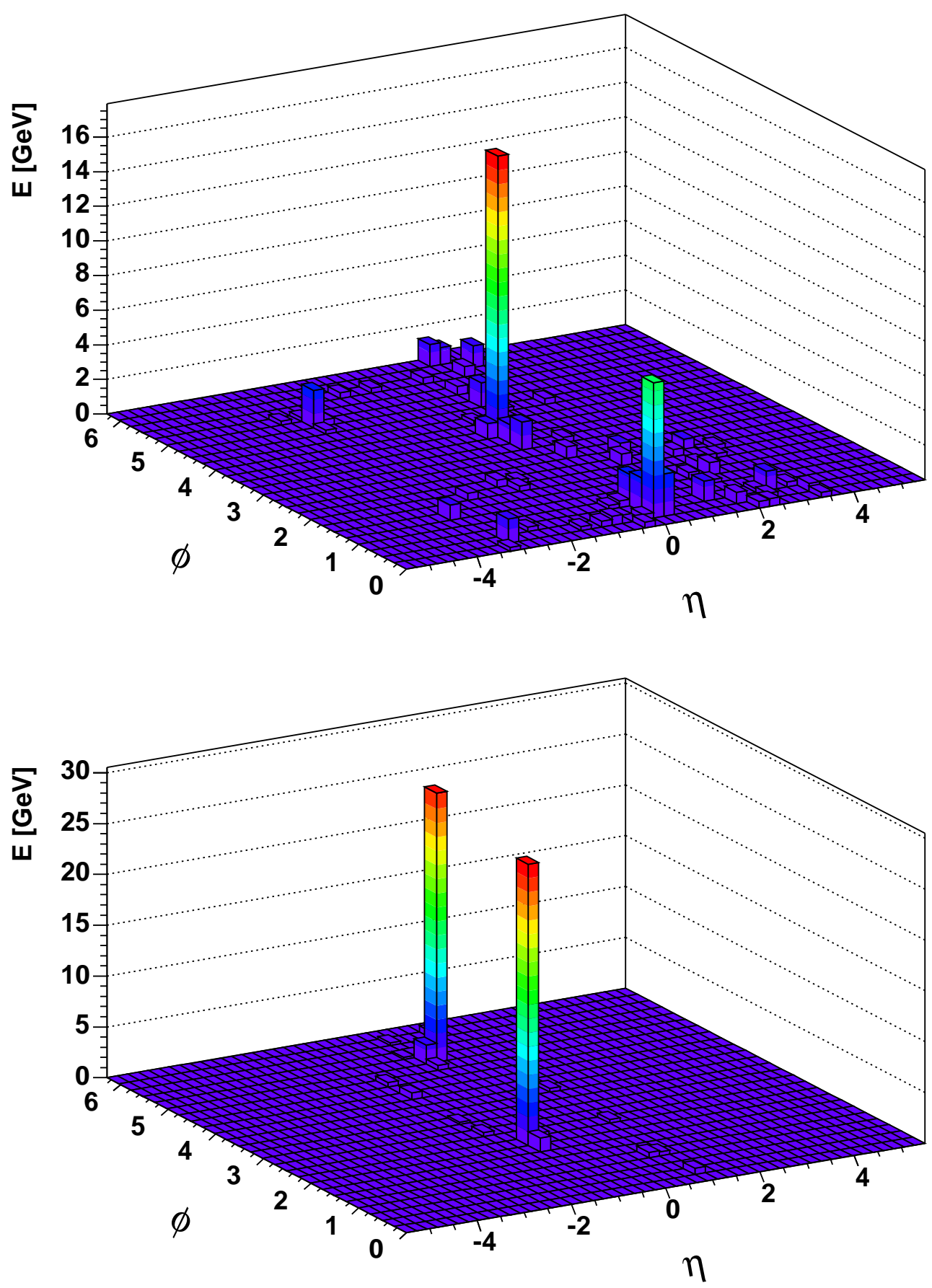

Figure 7.18: The energy deposition in the calorimeter from an event with $R_{j j}=0.68$ (top) and $R_{j j}=1$ (bottom) 


\subsection{Discussion}

Previous and on-going searches for central exclusive production made by the CDF collaboration have concentrated on the lower $R_{j j}$ region by requiring that the leading jet has $E_{T}$ of only $10 \mathrm{GeV}$. As illustrated in figure 4.24, using a low jet $E_{T}$ cut preserves many low $R_{j j}$ events, which makes the excess at high $R_{j j}$ difficult to observe above the background of double pomeron exchange. Further, CDF use forward proton taggers as a trigger criterion, which have acceptance only for $x>0.03$, whereas the expected $x_{1,2}$ values for a di-jet system in which both jets have $E_{T}=10 \mathrm{GeV}$ is around $x_{1,2} \simeq 0.01$.

Figures 7.12 to 7.14 all indicate that around 60 events have been found that do not fit the Pomwig expectation for the $R_{j j}$ distribution as $R_{j j} \rightarrow 1$. Although figure 7.8 shows that detector effects could explain the excess of high $R_{j j}$ events, the same figure also suggests that the $D \varnothing$ calorimeter would need to fail to detect particles with $4 \mathrm{GeV}$ of energy in order for this to be the case. Such a failure would impact even the precision on the top quark mass measurement [85]. Further, figures 7.9, 7.11 and 7.10 do not agree with a $4 \mathrm{GeV}$ threshold. Given that double pomeron exchange does not describe the data in the high $R_{j j}$ region, another process must be introduced in order to explain the data. As simulated by ExHuME, central exclusive production certainly can provide such an explanation and a fit for the excess of high $R_{j j}$ events. Although the high $R_{j j}$ events are not double pomeron exchange, whether they are truly exclusive may only be determined by also observing the intact outgoing protons.

In figures 7.12 and especially 7.13 there is a deficiency between the combined expectation for Pomwig and ExHuME and the data points at around $0.75<R_{j j}<$ 0.95. However, ExHuME currently lacks the $2 \rightarrow 3$ partonic process, which will almost certainly fill in the dip in the $R_{j j}$ distribution once it is added in the near future.

Further analysis, including a full detector simulation of the output from both Pomwig and ExHuME, is essential in order to prove conclusively that the excess events with large $R_{j j}$ truly are central exclusive. Note that using the true $x_{1,2}$, known either by using the Roman pots or by calibrating against a full detector 
simulation Monte Carlo, will result in a shift to the left in the $R_{j j}$ distributions of figure 7.12 of approximately 0.2 to 0.3 for the data points and both sets of Monte Carlo predictions. It will not be possible to determine the central exclusive cross section to any great accuracy without using the Roman pots, since calibrating the true $x_{1,2}$ with a full Monte Carlo simulation will invariably result in a $20-30 \%$ uncertainty (section 7.1.5).

If further analysis does show conclusive evidence for central exclusive production then distributions such as those in figures 7.15 and 7.17 should be able to distinguish between the KMR calculation implemented within ExHuME and other estimations $[59,86]$ for the central exclusive process. 


\section{Chapter 8}

\section{Concluding Remarks}

Central exclusive production could provide a uniquely clean environment in which to study QCD or as yet unobserved processes that can be mediated by gluon fusion. Chapter 3 gave details and predictions from two scenarios in which central exclusive production could be a crucial discovery channel for observing new phenomena. Central exclusive production could also prove invaluable in determining the quantum numbers of newly discovered states [87].

Having shown that central exclusive production is useful and interesting, it became necessary to produce a Monte Carlo simulation in order to make predictions for both the Tevatron and the LHC. ExHuME, detailed in chapter 4 and appendix A, provides the only current simulation of the KMR calculation of the central exclusive process.

The next few years promise to be both interesting and challenging for the study of central exclusive production. While the analysis presented here provides evidence for central exclusive production and similar analysis made by CDF also produced strong evidence, exclusive di-jets must yet be fully confirmed at the Tevatron by tagging the outgoing protons on both sides. The proposed FP420 project poses further challenges, noteably the lack of a diffractive trigger for either forward gaps or the proton taggers, but would surely return great rewards in terms of a clean measuring environment at the LHC. ExHuME should hopefully continue to be a useful tool for analysis at the Tevatron and for overcoming some of the challenges of FP420. 
One of the challenges faced by $D \varnothing$ was knowing the position of the proton taggers in the absence of an elastic trigger and, unlike FP420, without a beam position monitor. Chapter 6 explored the possibility of solving this problem using the pattern of proton hits in the pots themselves.

In order to validate the ExHuME simulation, a comparison was made to data taken at $\mathrm{D} \varnothing$ for central exclusive di-jet production. The data show that there are unexplained events with a high $R_{j j}$ measurement. This is evidence that central exclusive production is present and, further, ExHuME does not disagree with the experimental distributions in any significant way. Despite this, it cannot be concluded that central exclusive production has been observed and that ExHuME is the correct description because the outgoing protons were not observed and detector effects are not known completely. Crucially, however, ExHuME was able to inform the analysis that a higher jet $E_{\perp}$ cut and lower values of $x_{1,2}$ may lead to an improved separation between exclusive events and double Pomeron exchange compared to the analysis carried out at CDF. 


\section{Appendix A}

\section{The ExHuME Monte Carlo}

\section{A.1 Design}

ExHuME is written in a modular way using $\mathrm{C}++$. There are two main classes that are needed to generate central exclusive events. The first is a CrossSection class that calculates the differential luminosity, the gluon fusion sub-process and the kinematics of any outgoing particles. The second class needed is an Event class that generates the events.

\section{A.2 The CrossSection Class}

The CrossSection class exploits the factorisation of the cross section into a central sub-process and a differential luminosity calculation. CrossSection is an abstract base class containing the calculation of the effective luminosity of the gluon-gluon collision with a virtual method for the gluon fusion subprocess. Complete processes are created by inheriting from the CrossSection and explicitly defining a sub-process. This makes it relatively simple to implement new processes in addition to the currently implemented Standard Model Higgs, di-gluon and di-quark processes. 


\section{CrossSection Methods}

The constructor for the CrossSection class is

CrossSection(int, char**),

which allows the user to pass a card file from the command line that changes the default values used in the luminosity calculation (see Appendix A.5). The constructors for the derived classes are Higgs (int, char**), QQ (int, char**), GG (int, char**) or Dummy (int, char**) for Higgs, di-quark and di-gluon production respectively. Dummy does not calculate a sub-process and can be used to access the differential luminosity.

The invariant mass and rapidity of the central system and the momentum transfers and azimuthal angles, $\phi_{1,2}$, of the outgoing protons can be set by the CrossSection method

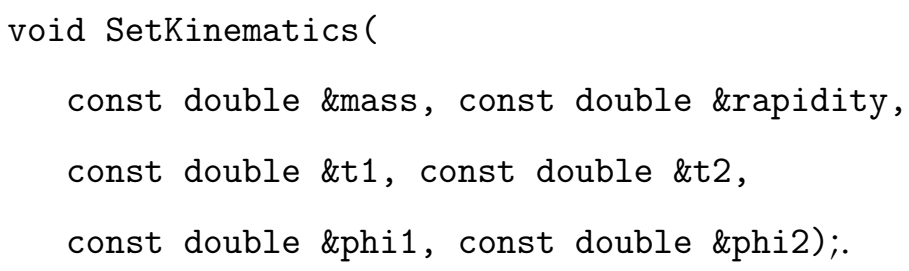

The method

double Differential()

then returns the differential cross section. The 4-vectors of the outgoing protons can be accessed by the methods

HepLorentzVector GetProton1() and

HepLorentzVector GetProton2().

The sub-process parton level information can be extracted by the method 
std: :vector $<$ Particle $>$ GetPartons()

which returns the outgoing particles from the gluon fusion matrix element prior to decay, parton showering and hadronisation. The Particle class that GetPartons () returns contains the particle momentum, p, the PDG ID code, id and vertex, vtx. It is also possible to access the kinematics of the cross section by the following methods:

double GetRoot_s();

Returns the invariant mass of the colliding beams.

double GetsHat();

Returns the invariant mass squared of the central system.

double GetSqrtsHat();

Returns the invariant mass of the central system.

double Getx1();

Gets the value of $x_{1}$.

double Getx2();

Gets the value of $x_{2}$

double Gett1();

Gets the value of $t_{1}$.

double Gett2();

Gets the value of $t_{2}$. 
double GetPhi1();

Gets the value of $\phi_{1}$, the azimuthal angle between the outgoing proton 1 and the $x$ direction.

double GetPhi2();

Gets the value of $\phi_{2}$, the azimuthal angle between the outgoing proton 2 and the $x$ direction.

double GetEta();

Gets the rapidity, $y$, of the central system.

Hadronisation is performed via the method

void Hadronise()

which places the event record into an external hepevt common block that can be written to file.

There are a number of sub-process specific methods. The Higgs decay type can be set by the method

SetHiggsDecay (const int\&)

with the PDG code of the decay products as the argument. Similarly the quark type in $q \bar{q}$ can be set by the method

SetQuarkType(const int\&)

which defaults to $b \bar{b}$ production. In addition, the GG and QQ classes contain the method

SetThetaMin (const double\&) 
which sets the minimum (and maximum) polar angle of an outgoing parton relative to the beamline in the rest frame of the central system. The default value is $\cos \theta=0.95$.

\section{A.3 The Event Class}

The Event class generates events, calculates the total cross section and reports the efficiency with which events were generated once event generation has finished. Event contains a pointer to a CrossSection and the user specifies which subprocess is to be generated in the constructor for the Event. A weighting algorithm is initialised and used to return the mass distribution of the differential cross section at a rapidity zero and is effective even for a narrow resonance such as the Higgs. The variables $t_{1}$ and $t_{2}$ are distributed according to $e^{b\left(t_{1}+t_{2}\right)}$ whilst $\phi_{1}, \phi_{2}$ and $y$ are uniformly distributed.

\section{Event Methods}

The event is defined by calling the constructor

Event(CrossSection\& $P$, const unsigned int $R$ ),

where $\mathrm{P}$ is a CrossSection with the sub-process defined and $\mathrm{R}$ is a random number seed. There are a number of methods that can be used to set the kinematic ranges of the parameters used to define the event:

void Setx1Max (const double\&);

Sets the upper limit of $x_{1}$

void Setx2Max (const double\&);

Sets the upper limit of $x_{2}$. 
void Sett1Max (const double\&);

Sets the upper limit of $t_{1}(t \leq 0)$.

void Sett1Min(const double\&);

Sets the lower limit of $t_{1}$.

void Sett2Max (const double\&);

Sets the upper limit of $t_{2}$.

void Sett2Min(const double\&);

Sets the lower limit of $t_{2}$.

void SetMassRange(const double \& minimum, const double \& maximum);

Sets the lower and upper limits of the mass range respectively.

The last method that is used before event generation is

void SetParameterSpace(),

which must be called in order to initialise the event generation. Individual events are generated by the method

void Generate().

Finally, the total cross section from the events generated can be calculated by the method

double CrossSectionCalculation()

and the efficiency via 
double GetEfficiency().

\section{A.4 Using ExHuME}

\section{Installing ExHuME}

The current version of ExHuME is 1.3.2, ( major release . physics processes . bug fixes ) and is available from [88] or on request from the authors. In its standard form ExHuME must be linked at compilation to Pythia [56], CLHEP [89] and either LHAPDF [51] or the CERN PDFLIB [90]. It would also be possible to modify ExHuME to use Herwig [91] instead of Pythia for the hadronisation or to use a stand alone PDF instead of either LHAPDF or PDFLIB. By default ExHuME sets the location of the directory containing the grid or parameter files for LHAPDF to be wherever the program is executed from. A symbolic link should be created to wherever the grid and parameter files actually reside. For further information please see the respective documentation for each of these programs .

\section{Example Main Program}

In this section we demonstrate a simple main program that generates 5000 $H \rightarrow W W^{*}$ events for a Higgs with the default mass of $120 \mathrm{GeV}$. We also show how to extract simple information from the hepevt record.

The following headers are for ExHuME:

\#include "Event.h"

\#include "Higgs.h"

int main(int argc, char** argv) \{

Declare a new Higgs CrossSection:

Exhume: :Higgs higgs (argc,argv); 
and set the Higgs to decay only to W bosons:

higgs.SetHiggsDecay(24); // 24 is the PDG code for W

Declare an event with the Higgs as the cross section and a random number seed of 1111.

Exhume: :Event HiggsEvent (higgs,1111);

The allowed range of gluon fusion invariant masses must be set. As long as the range is much bigger than the width of the resonance the results will not be sensitive to the range chosen. This is not the case whenever the central system does not have a narrow width, for example in di-gluon and di-quark production. In this example we set the mass range to be between 115 and $125 \mathrm{GeV}$, some 3 orders of magnitude larger than the Standard Model Higgs Boson width at 0.0036 GeV.

HiggsEvent. SetMassRange $(115,125)$;

This must be called before event generation can begin:

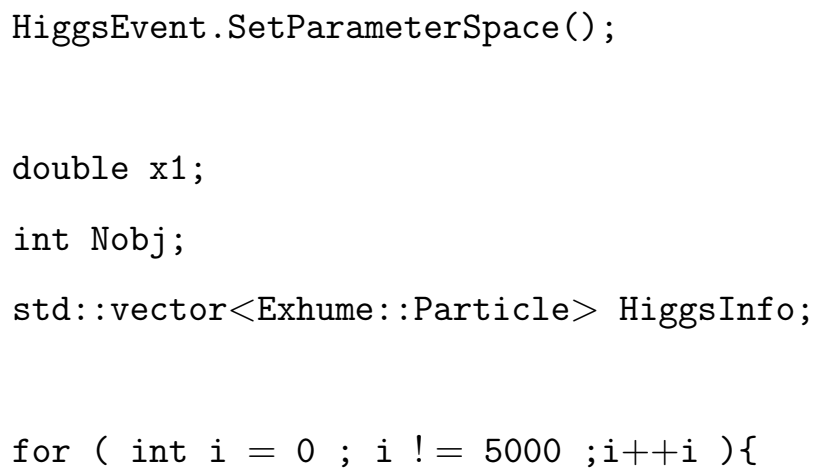

The next line generates a single event: 
HiggsEvent.Generate();

Access the longitudinal momentum loss of proton 1 for this event:

$$
\mathrm{x} 1=\text { process.Getx1(); }
$$

and the information about the Higgs:

$$
\text { HiggsInfo }=\text { process.GetPartons }() \text {; }
$$

Get the number of particles in the hepevt common block:

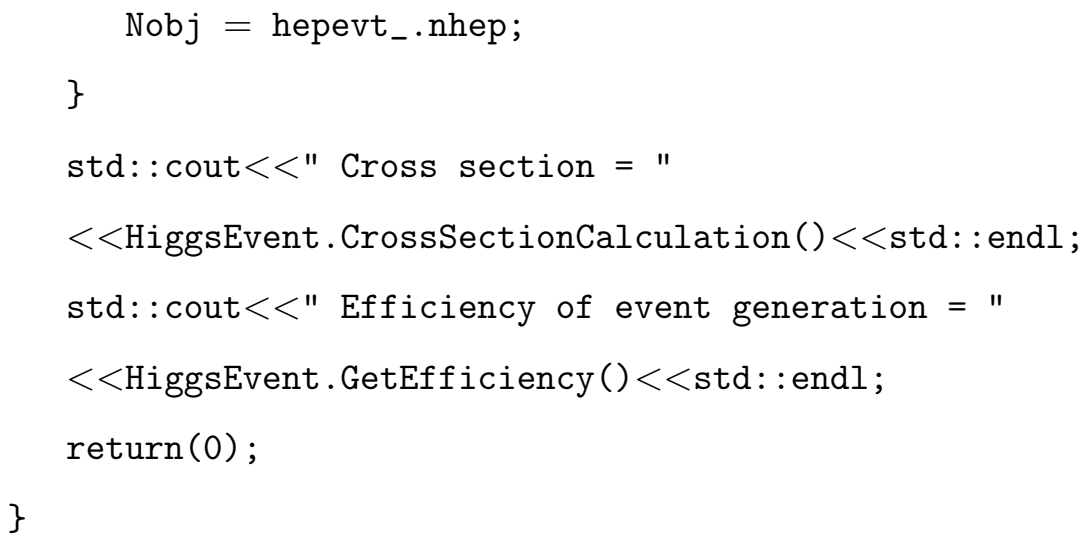

The program allows (but does not demand) a card file to be given on the command line that overrides the default parameters. Such a card file could look like

$\begin{array}{lr}\text { HiggsMass } & 140 \\ \text { TopMass } & 180\end{array}$

which would be appropriate for investigating the effects of varying the higgs and top masses. 


\section{A.5 The ExHuME Control Parameters}

ExHuME can be controlled by passing a card file from the command line that can contain the control parameters given in table A.1. The collider can be set to the LHC (1), the Tevatron (0) or neither $(-1)$. Choosing a collider sets the proton collision energy, $\sqrt{s}$, the survival factor $\mathcal{S}^{2}$ and $R_{g}$, which accounts for the skewed effect in the un-integrated gluons. Choosing neither means that the user must set these parameters. The PDF values are the PDF set numbers accepted by the LHAPDF library. Freeze is a scale below which $\alpha_{s}$ is frozen.

\begin{tabular}{|l|l|l|l|}
\hline Parameter & Name & Type & Default \\
\hline$\alpha$ & AlphaEW & double & 0.0072974 \\
$M_{W}$ & WMass & double & 80.33 \\
$M_{Z}$ & ZMass & double & 91.127 \\
$M_{H}$ & HiggsMass & double & 120.0 \\
$M_{t}$ & TopMass & double & 175.0 \\
$M_{b}$ & BottomMass & double & 4.6 \\
$M_{c}$ & CharmMass & double & 1.42 \\
$M_{s}$ & StrangeMass & double & 0.19 \\
$M_{\tau}$ & TauMass & double & 1.77 \\
$M_{\mu}$ & MuonMass & double & 0.1057 \\
$v$ & HiggsVev & double & 246.0 \\
$Q_{\perp \text { min }}^{2}$ & MinQt2 & double & 0.64 \\
$\Lambda_{Q C D}(\mathrm{MeV})$ & LambdaQCD & double & $80(\mathrm{MeV})$ \\
& Freeze & double & $Q \perp$ min \\
$b$ & B & double & 4.0 \\
collider & FNAL_or_LHC & int & 1 \\
$s$ & s & double & $1.96 \times 10^{8}$ \\
$s^{\frac{1}{2}}$ & root_s & double & 14000.0 \\
$R_{g}$ & Rg & double & 1.2 \\
$\mathcal{S}^{2}$ & Survive & double & 0.03 \\
$P D F$ & PDF & int & 20250 \\
\hline
\end{tabular}

Table A.1: ExHuME control parameters 


\section{Bibliography}

[1] A. D. Martin, M. G. Ryskin, and V. A. Khoze, "Dijet hadroproduction with rapidity gaps and QCD double logarithmic effects," Phys. Rev., vol. D56, pp. 5867-5874, 1997.

[2] V. A. Khoze, A. D. Martin, and M. G. Ryskin, "Prospects for new physics observations in diffractive processes at the LHC and tevatron," Eur. Phys. J., vol. C23, pp. 311-327, 2002. hep-ph/0111078.

[3] V. A. Khoze, A. D. Martin, and M. G. Ryskin, "Physics with tagged forward protons at the LHC," Eur. Phys. J., vol. C24, pp. 581-587, 2002. hepph/0203122.

[4] A. B. Kaidalov, V. A. Khoze, A. D. Martin, and M. G. Ryskin, "Extending the study of the Higgs sector at the LHC by proton tagging," Eur. Phys. J., vol. C33, pp. 261-271, 2004.

[5] M. G. Albrow and A. Rostovtsev, "Searching for the Higgs at hadron colliders using the missing mass method," 2000. hep-ph/0009336.

[6] M. Carena, J. R. Ellis, A. Pilaftsis, and C. E. M. Wagner, "CP-violating MSSM Higgs bosons in the light of LEP 2," Phys. Lett., vol. B495, pp. 155-163, 2000. hep-ph/0009212.

[7] L. Randall and R. Sundrum, "A large mass hierarchy from a small extra dimension," Phys. Rev. Lett., vol. 83, pp. 3370-3373, 1999.

[8] M. Convery, M. Gallinaro, K. Goulianos, and C. Mesropian, "Dpe and exclusive dijet productions in run II," http://wwwcdf.fnal.gov/physics/new/qcd/QCD.html.

[9] M. Albrow, B. Caron, A. Hamilton, B. Heinemann, and J. Pinfold, "Evidence of exclusive $\gamma \gamma$ production in hadron hadron collisions," 2006. CDF note 8226.

[10] J. Monk and A. Pilkington, "ExHuME: A Monte Carlo event generator for exclusive diffraction," Comput. Phys. Commun., vol. 175, pp. 232-239, 2006. hep-ph/0502077.

[11] S. P. Weinberg, "Gravitation and Cosmology: Principles and applications of the general theory of relativity," 1972. John Wiley and Sons Inc 657p. 
[12] I. J. R. Aitchison and A. J. G. Hey, "Gauge theories in particle physics: A practical introduction," BRISTOL, UK: HILGER (1989) 571p.

[13] The Particle Data Group 2006.

[14] S. Fukuda et al., "Tau neutrinos favored over sterile neutrinos in atmospheric muon neutrino oscillations," Phys. Rev. Lett., vol. 85, pp. 3999-4003, 2000. hep-ex/0009001.

[15] P. Bock et al., "Lower bound for the standard model Higgs boson mass from combining the results of the four LEP experiments," CERN-EP-98-046.

[16] R. K. Ellis, W. J. Stirling, and B. R. Webber, "QCD and collider physics," Camb. Monogr. Part. Phys. Nucl. Phys. Cosmol., vol. 8, pp. 1-435, 1996.

[17] M. E. Peskin and D. V. Schroeder, "An introduction to quantum field theory," Reading, USA: Addison-Wesley (1995) 842 p.

[18] S. P. Martin, "A supersymmetry primer," 1997. hep-ph/9709356.

[19] G. Altarelli and G. Parisi, "Asymptotic freedom in parton language," Nucl. Phys., vol. B126, p. 298, 1977.

[20] V. N. Gribov and L. N. Lipatov, "Deep inelastic ep scattering in perturbation theory," Sov.J.Nucl.Phys., p. 438, 1972.

[21] Y. L. Dokshitzer Sov.Phys. JETP, p. 641, 1977.

[22] C. Gwenlan, "The structure of the proton and NLO QCD fits," PoS, vol. HEP2005, p. 041, 2006. hep-ex/0511055.

[23] A. D. Martin, R. G. Roberts, W. J. Stirling, and R. S. Thorne, "MRST2001: Partons and alpha(s) from precise deep inelastic scattering and Tevatron jet data," Eur. Phys. J., vol. C23, pp. 73-87, 2002. hep-ph/0110215.

[24] H. L. Lai et al., "Global QCD analysis and the CTEQ parton distributions," Phys. Rev., vol. D51, pp. 4763-4782, 1995. hep-ph/9410404.

[25] J. R. Forshaw and D. A. Ross, "Quantum chromodynamics and the pomeron," Cambridge Lect. Notes Phys., vol. 9, pp. 1-248, 1997.

[26] S. Alekhin et al., "HERA and the LHC - a workshop on the implications of HERA for LHC physics: Proceedings part B," 2005. hep-ph/0601013.

[27] J. R. Forshaw, "Diffractive higgs production: Theory," 2005. hep$\mathrm{ph} / 0508274$.

[28] A. G. Shuvaev, K. J. Golec-Biernat, A. D. Martin, and M. G. Ryskin, "Offdiagonal distributions fixed by diagonal partons at small $\mathrm{x}$ and $\mathrm{xi}^{\mathrm{i}}$ " Phys. Rev., vol. D60, p. 014015, 1999.

[29] B. E. Cox, "A review of forward proton tagging at 420-m at the Ihc, and relevant results from the tevatron and hera," AIP Conf. Proc., vol. 753, pp. 103-111, 2005. 
[30] M. G. Albrow et al., "FP420: An R \& D proposal to investigate the feasibility of installing proton tagging detectors in the 420-m region at LHC," CERN publishing, 2005. CERN-LHCC-2005-025.

[31] S. M. Barr, G. Segre, and H. A. Weldon, "The magnitude of the cosmological baryon asymmetry," Phys. Rev., vol. D20, p. 2494, 1979.

[32] M. Carena, J. R. Ellis, A. Pilaftsis, and C. E. M. Wagner, "Higgs-boson pole masses in the MSSM with explicit CP violation," Nucl. Phys., vol. B625, pp. 345-371, 2002. hep-ph/0111245.

[33] M. Carena, J. R. Ellis, A. Pilaftsis, and C. E. M. Wagner, "Renormalizationgroup-improved effective potential for the mssm higgs sector with explicit cp violation," Nucl. Phys., vol. B586, pp. 92-140, 2000. hep-ph/0003180.

[34] M. Carena, J. R. Ellis, S. Mrenna, A. Pilaftsis, and C. E. M. Wagner, "Collider probes of the mssm higgs sector with explicit cp violation," Nucl. Phys., vol. B659, pp. 145-178, 2003. hep-ph/0211467.

[35] S. Y. Choi and J. S. Lee, "Mssm higgs-boson production at hadron colliders with explicit cp violation," Phys. Rev., vol. D61, p. 115002, 2000. hepph/9910557.

[36] S. Y. Choi and J. S. Lee, "Probing mssm higgs sector with explicit cp violation at a photon linear collider," Phys. Rev., vol. D62, p. 036005, 2000. hep$\mathrm{ph} / 9912330$.

[37] S. Y. Choi, K. Hagiwara, and J. S. Lee, "Observability of the lightest mssm higgs boson with explicit cp violation via gluon fusion at the lhc," Phys. Lett., vol. B529, pp. 212-221, 2002. hep-ph/0110138.

[38] A. Pilaftsis The Fortran code cph.f is available at http://home.cern.ch/p/pilaftsi/www/.

[39] B. E. Cox, J. R. Forshaw, J. S. Lee, J. Monk, and A. Pilaftsis, "Observing a light CP-violating Higgs boson in diffraction," Phys. Rev., vol. D68, p. 075004, 2003. hep-ph/0303206.

[40] V. A. Khoze, A. D. Martin, and M. G. Ryskin, "Soft diffraction and the elastic slope at Tevatron and LHC energies: A multi-pomeron approach," Eur. Phys. J., vol. C18, pp. 167-179, 2000. hep-ph/0007359.

[41] L. Randall and R. Sundrum, "An alternative to compactification," Phys. Rev. Lett., vol. 83, pp. 4690-4693, 1999.

[42] W. D. Goldberger and M. B. Wise, "Phenomenology of a stabilized modulus," Phys. Lett., vol. B475, pp. 275-279, 2000.

[43] D. Dominici, B. Grzadkowski, J. F. Gunion, and M. Toharia, "The scalar sector of the Randall-Sundrum model," Nucl. Phys., vol. B671, pp. 243292, 2003. hep-ph/0206192. 
[44] C. Csaki, M. Graesser, and G. D. Kribbs, "Radion dynamics and electroweak physics," hep-ph/0008151.

[45] C. Csaki, M. Graesser, L. Randall, and J. Terning, "Cosmology of brane models with radion stabilization," Phys. Rev., vol. D62, p. 045015, 2000.

[46] Private communication, J. S. Lee.

[47] V. A. Khoze, A. D. Martin, and M. G. Ryskin, "Can the higgs be seen in rapidity gap events at the tevatron or the Ihc?," Eur. Phys. J., vol. C14, pp. 525-534, 2000. hep-ph/0002072.

[48] A. De Roeck, V. A. Khoze, A. D. Martin, R. Orava, and M. G. Ryskin, "Ways to detect a light Higgs boson at the LHC," Eur. Phys. J., vol. C25, pp. 391-403, 2002. hep-ph/0207042.

[49] M. Goossens et al., "CERNLIB: Short writeups," Computing and Networks Division, CERN, 1996. http://wwwinfo.cern.ch/asdoc/psdir/shortwrups.dir/cernlib.ps.gz.

[50] Private Communication, M. Ryskin

[51] M. R. Whalley, D. Bourilkov, and R. C. Group, "The Les houches accord PDFs (LHAPDF) and LHAGLUE," 2005. hep-ph/0508110.

[52] M. A. Kimber, A. D. Martin, and M. G. Ryskin, "Unintegrated parton distributions and prompt photon hadroproduction," Eur. Phys. J., vol. C12, pp. 655-661, 2000. hep-ph/9911379.

[53] P. J. Bussey, T. D. Coughlin, J. R. Forshaw, and A. D. Pilkington, "Central exclusive production of longlived gluinos at the Ihc," 2006. hep-ph/0607264.

[54] A. Djouadi, J. Kalinowski, and M. Spira, "HDECAY: A program for Higgs boson decays in the standard model and its supersymmetric extension," Comput. Phys. Commun., vol. 108, pp. 56-74, 1998. hep-ph/9704448.

[55] M. H. Seymour, "The Higgs boson line shape and perturbative unitarity," Phys. Lett., vol. B354, pp. 409-414, 1995. hep-ph/9505211.

[56] T. Sjostrand, L. Lonnblad, and S. Mrenna, "PYTHIA 6.2: Physics and manual," 2001. hep-ph/0108264.

[57] B. E. Cox et al., "Detecting the standard model higgs boson in the w w decay channel using forward proton tagging at the Ihc," Eur. Phys. J., vol. C45, pp. 401-407, 2006. hep-ph/0505240.

[58] J. Kalliopuska et al., "Totem forward measurements: exclusive central production," HERA and the LHC - A workshop on the implications of HERA for LHC physics: Proceedings Part B, 2005. hep-ph/0601012.

[59] M. Boonekamp and T. Kucs, "DPEMC: A Monte-Carlo for double diffraction," Comput. Phys. Commun., vol. 167, p. 217, 2005. hep-ph/0312273. 
[60] J. Bromley et al., "Hztool: A package for monte carlo-data comparison at hera (version 1.0)," Prepared for Workshop on Future Physics at HERA (Preceded by meetings 25-26 Sep 1995 and 7-9 Feb 1996 at DESY), Hamburg, Germany, 30-31 May 1996.

[61] V. A. Khoze, M. G. Ryskin, and W. J. Stirling, "On radiative qcd backgrounds to exclusive $\mathrm{h} \rightarrow>\mathrm{b}$ anti-b production at the lhc and a photon collider," 2006. hep-ph/0607134.

[62] A. Wyatt et al., "Exclusive $\chi_{c}$ production," http://wwwcdf.fnal.gov/physics/new/qcd/abstracts/ExcluChic_03.html.

[63] http://www-visualmedia.fnal.gov/VMS_Site/gallery/stillphotos/2004/0400/040436D.hr.jpg.

[64] V. M. Abazov et al., "The upgraded DØ detector," 2005. physics/0507191.

[65] M. R. for the DØC̃ollaboration, "The silicon microstrip tracker for the DØ upgrade," DØ Note 3553.

[66] J. Kotcher, "Design, performance and upgrade of the DØ calorimeter," $D \varnothing$ Note 2417, 1995.

[67] B. B. et. al., "Technical design of the central muon system," DØ Note 3365, 1998.

[68] V. Abramov et al., "Technical design report for the DØ forward trigger scintillation counters," DØ Note 3237, 1997.

[69] A. Brandt., "D $\varnothing$ diffraction/forward proton detector update," Manchester forward physics workshop, 2004.

[70] J. Annala, B. Hanna, T. Johnson, D. Still, et al., "Investigation of the Tevatron 16 house quench of 12/5/2003," 2003. FNAL accelerator division note.

[71] M. Strang, "Detector numbering scheme," http://wwwd0.fnal.gov/ gilvan/Fpd/detector.html.

[72] R. Brun and F. Rademakers, "ROOT: An object oriented data analysis framework," Nucl. Instrum. Meth., vol. A389, pp. 81-86, 1997.

[73] M. P. A. Fitzgibbon and R. B. Fisher., "Direct least square fitting of ellipses," Tern Analysis and Machine Intelligence., vol. 21, no. 5, pp. 476-480, 1999.

[74] J. Molina, "Study of elastic scattering at $\sqrt{s}=1.96$ tev with the forward proton detector," FERMILAB-THESIS-2003-18.

[75] B. Matei and P. Meer, "Reduction of bias in maximum likelihood ellipse fitting," 2000. citeseer.ist.psu.edu/article/matei00reduction.html.

[76] J. C. Collins, "Light-cone variables, rapidity and all that," 1997. hep$\mathrm{ph} / 9705393$. 
[77] A. Donnachie and P. V. Landshoff, "Total cross-sections," Phys. Lett., vol. B296, pp. 227-232, 1992. hep-ph/9209205.

[78] B. E. Cox and J. R. Forshaw, "POMWIG: HERWIG for diffractive interactions," Comput. Phys. Commun., vol. 144, pp. 104-110, 2002. hep$\mathrm{ph} / 0010303$.

[79] Private communication, J. Stark by way of Tamsin Edwards.

[80] T. L. Edwards, "Diffractively produced z bosons in the muon decay channel in $p$ anti-p collisions at $s^{* *}(1 / 2)=1.96-\mathrm{tev}$, and the measurement of the efficiency of the d0 run ii luminosity monitor," FERMILAB-THESIS-200605.

[81] M. C. Cousinou and P. Verdier, "Missing ET run/LBN selection," http://wwwd0.fnal.gov/computing/data_quality/d0_private/caloff_temp/p17preliminary.php.

[82] B. Abbott et al., "Determination of the absolute jet energy scale in the DØ calorimeters," Nucl. Instrum. Meth., vol. A424, pp. 352-394, 1999. hep-ex/9805009.

[83] B. Cox, J. R. Forshaw, and L. Lonnblad, "Hard colour singlet exchange at the Tevatron," JHEP, vol. 10, p. 023, 1999. hep-ph/9908464.

[84] B. E. Cox, J. R. Forshaw, and L. Lonnblad, "Is BFKL ruled out by the Tevatron gaps between jets data?," 1999. hep-ph/9912489.

[85] "Measurement of the top quark mass in the lepton + jets final state with the matrix element method," Phys. Rev., vol. D74, p. 092005, 2006. hepex/0609053.

[86] R. A. Ryutin, "Edde monte carlo event generator," 2004.

[87] J. R. Ellis, J. S. Lee, and A. Pilaftsis, "Diffraction as a ср and lineshape analyzer for mssm higgs bosons at the lhc," Phys. Rev., vol. D71, p. 075007, 2005. hep-ph/0502251.

[88] http://hepforge.cedar.ac.uk/exhume/.

[89] L. Lonnblad, "CLHEP: A project for designing a C++ class library for highenergy physics," Comput. Phys. Commun., vol. 84, pp. 307-316, 1994. 307-316.

[90] H. Plothow-Besch, "PDFLIB: A library of all available parton density functions of the nucleon, the pion and the photon and the corresponding alpha-s calculations," Comput. Phys. Commun., vol. 75, pp. 396-416, 1993.

[91] G. Corcella et al., "HERWIG 6: An event generator for hadron emission reactions with interfering gluons (including supersymmetric processes)," JHEP, vol. 01, p. 010, 2001. hep-ph/0011363. 